\title{
Two-Parameter Fracture Mechanics Analysis of Mixed-Mode Specimens Under Static and Fatigue Loading
}

\author{
by
}

\author{
Qays N. Nazarali \\ B.Eng.
}

\begin{abstract}
A thesis submitted to
the Faculty of Graduate Studies and Research

in partial fulfillment of

the requirements for the degree of

Master of Applied Science (Engineering)
\end{abstract}

Ottawa-Carleton Institute for Mechanical and Aerospace Engineering

Department of Mechanical and Aerospace Engineering

Carleton University

Ottawa, Ontario

Canada

September 2008C

Q. Nazarali 


$\begin{array}{ll}\begin{array}{l}\text { Library and } \\ \text { Archives Canada }\end{array} & \begin{array}{l}\text { Bibliothèque et } \\ \text { Archives Canada }\end{array} \\ \begin{array}{l}\text { Published Heritage } \\ \text { Branch }\end{array} & \begin{array}{l}\text { Direction du } \\ \text { Patrimoine de l'édition }\end{array} \\ \begin{array}{l}\text { 395 Wellington Street } \\ \text { Ottawa ON K1A 0N4 } \\ \text { Canada }\end{array} & \begin{array}{l}\text { 395, rue Wellington } \\ \text { Ottawa ON K1A 0N4 } \\ \text { Canada }\end{array}\end{array}$

Your file Votre référence ISBN: 978-0-494-47520-1

Our file Notre référence

ISBN: 978-0-494-47520-1

NOTICE:

The author has granted a nonexclusive license allowing Library and Archives Canada to reproduce, publish, archive, preserve, conserve, communicate to the public by telecommunication or on the Internet, loan, distribute and sell theses worldwide, for commercial or noncommercial purposes, in microform, paper, electronic and/or any other formats.

The author retains copyright ownership and moral rights in this thesis. Neither the thesis nor substantial extracts from it may be printed or otherwise reproduced without the author's permission.
AVIS:

L'auteur a accordé une licence non exclusive permettant à la Bibliothèque et Archives Canada de reproduire, publier, archiver, sauvegarder, conserver, transmettre au public par télécommunication ou par l'Internet, prêter, distribuer et vendre des thèses partout dans le monde, à des fins commerciales ou autres, sur support microforme, papier, électronique et/ou autres formats.

L'auteur conserve la propriété du droit d'auteur et des droits moraux qui protège cette thèse. $\mathrm{Ni}$ la thèse ni des extraits substantiels de celle-ci ne doivent être imprimés ou autrement reproduits sans son autorisation.
In compliance with the Canadian Privacy Act some supporting forms may have been removed from this thesis.

While these forms may be included in the document page count, their removal does not represent any loss of content from the thesis.
Conformément à la loi canadienne sur la protection de la vie privée, quelques formulaires secondaires ont été enlevés de cette thèse.

Bien que ces formulaires aient inclus dans la pagination, il n'y aura aucun contenu manquant.

\section{Canada}




\section{Abstract}

The T-stress is a constant stress that acts parallel to the crack-front. In twoparameter fracture mechanics, the $\mathrm{T}$-stress in conjunction with the stress intensity factor (SIF) is an important parameter for characterizing the stress state in the vicinity of a crack-tip. Due to its importance in characterizing the crack-tip stress field, T-stress values were calculated using finite element analysis (FEA) for a center cracked plate (CCP) and single-edge cracked plate (SECP) specimen. The T-stress values for both the CCP and SECP specimens were determined for cracks with relative sizes varying from 0.1 to 0.8 with respect to the specimen width and oriented at various angles between $0^{\circ}$ and $90^{\circ}$ with respect to the horizontal.

The crack-tip stress field is defined in terms of both the SIF and the T-stress using the William's series expansion [Williams, 1957]. The crack-tip stress field is incorporated into the Von Mises yield formula to develop an expression that models the crack-tip plastic zone under static mixed-mode loading conditions influenced by $\mathrm{T}$-stress. Using the static plastic zone expression, the plastic zone is mapped and analyzed for various combinations of mode II to mode I SIF ratios and T-stress. The static plastic zone expression enables the characterization of constraint and fracture resistance under mixedmode conditions.

The static plastic zone expression is adapted for cyclic loading conditions and used to develop a fatigue crack propagation model that takes into account the effect of both mixed-mode loading and T-stress. Finally, the cyclic plastic zone expression is used to demonstrate the significance of T-stress when predicting fatigue crack growth. 


\section{Acknowledgements}

I would like to express my sincere appreciation to Dr. Xin Wang for this great opportunity to further pursue my education in the field of engineering, as well as the guidance, encouragement, and effort he put into my research and this thesis.

Financial support from the Natural Sciences and Engineering Research Council of Canada (NSERC) and the Ontario Centres of Excellence (OCE), Centre of Materials and Manufacturing, in the form of research grants to Dr. Xin Wang is appreciated.

I am indebted to the unsung heroes of the MAAE department: Marlene Groves and Nancy Powell, for the time, knowledge, effort, and patience they put into making my life as a graduate student that much more easier.

Most importantly, I would like to thank my parents for their love and support, my brother for his patience and understanding, my sister for her encouragement and insight, and last but not least, I would like to thank Connie for her unique perspective and being by my side through thick and thin. 


\section{Table of Contents}

Title Page $\quad$ i

Acceptance Sheet $\quad$ ii

Abstract

Acknowledgements iv

Table of Contents $\quad$ V

List of Tables viii

List of Figures $\quad$ xi

List of Appendices $\quad \mathrm{XV}$

Nomenclature $\quad$ xvi

Chapter 1: Introduction 1

1.1 Objective of Thesis 3

1.2 Outline of Thesis $\quad 4$

Figures $\quad 6$

Chapter 2: Background and Literature Review 7

2.1 Fracture Mechanics 7

2.1.1 Single-Parameter Fracture Mechanics $\quad 8$

2.1.2 Two-Parameter Fracture Mechanics $\quad 10$

2.1.3 Mixed-Mode Two-Parameter Fracture Mechanics 11

2.2 The Plastic Zone under Static Loading 13

2.3 Crack Propagation Analysis using The Plastic Zone under Cyclic Loading 15

2.4 The Finite Element Method $\quad 18$

$\begin{array}{ll}\text { 2.4.1 The Crack-Tip Stress Singularity } & 18\end{array}$

2.4.2 Mixed Mode Stress Intensity Factors and T-Stress 19

2.5 Mixed-Mode Test Specimens $\quad 23$

2.6 Concluding Remarks $\quad 25$

$\begin{array}{lr}\text { Figures } & 26\end{array}$ 
Chapter 3: Stress Intensity Factor and T-Stress Solutions for Mixed-Mode Test Specimens Subjected to Remote Tension Loading 36

3.1 Specimen Geometries $\quad 36$

3.2 Finite Element Analysis (FEA) 37

$\begin{array}{ll}3.2 .1 \text { FEA Modeling } & 37\end{array}$

3.2.2 Validation of FEA Model 38

$\begin{array}{ll}3.3 \text { Results and Discussion } & 40\end{array}$

3.3.1 CCP Specimen 41

3.3.2 SECP Specimen $\quad 42$

3.4 Conclusions $\quad 44$

$\begin{array}{ll}\text { Tables } & 45\end{array}$

Figures $\quad 53$

Chapter 4: Crack-Tip Plastic Zone Analysis Under Static Mixed-Mode $\begin{array}{ll}\text { Conditions } & 68\end{array}$

4.1 The Plastic Zone $\quad 68$

4.1.1 Modeling the Plastic Zone 68

4.1.2 Comparison of the Plastic Zone Model with Special Cases in

$\begin{array}{ll}\text { Literature } & 71\end{array}$

4.2 Plastic Zone Results $\quad 73$

4.2.1 Mapping the Plastic Zone 73

4.2.2 Discussions

$\begin{array}{ll}\text { 4.2.3 Continuity of Plastic Zone Boundary } & 77\end{array}$

4.3 Maximum Plastic Zone Size Results $\quad 78$

4.4 Characterizing Fracture Resistance $\quad 81$

$\begin{array}{ll}4.5 \text { Conclusion } & 84\end{array}$

Tables $\quad 85$

$\begin{array}{ll}\text { Figures } & 96\end{array}$ 
Chapter 5: Crack-Tip Plastic Zone Analysis Under Cyclic Mixed-Mode Conditions

5.1 The Cyclic Plastic Zone and Crack Propagation Rates 135

5.1.1 The Cyclic Plastic Zone 135

5.1.2 Crack Propagation Rate Model 137

5.2 Application of the Cyclic Plastic Zone to a Test Specimen 141

5.2.1 The Test Specimen 141

5.2.2 Determining Stress Intensity Factors and T-Stress for the Test Specimen 142

5.2.3 The Cyclic Plastic Zone for the Test Specimen 143

5.3 Discussion 145

5.4 Conclusion $\quad 146$

Tables 148

$\begin{array}{ll}\text { Figures } & 154\end{array}$

Chapter 6: Conclusions and Recommendations $\quad 170$

$\begin{array}{ll}6.1 \text { Conclusions } & 170\end{array}$

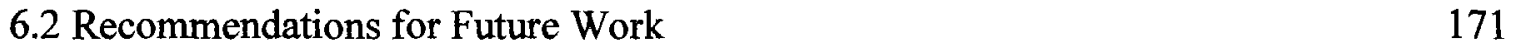

$\begin{array}{ll}\text { Appendices } & 173\end{array}$

$\begin{array}{ll}\text { References } & 194\end{array}$ 


\section{List of Tables}

Chapter 3: Stress Intensity Factor and T-Stress Solutions for Mixed-Mode Test Specimens Subjected to Remote Tension Loading 36

Table 3.1: Comparison of $f_{l}$ values from coarse mesh FEA and Kitagawa \&

Yuuki [1977] for the CCP specimen

Table 3.2: Comparison of $f_{2}$ values from coarse mesh FEA and Kitagawa \& Yuuki [1977] for the CCP specimen

Table 3.3: Comparison of $t$ values from coarse mesh FEA and Wang [2001] for the CCP specimen

Table 3.4: Comparison of $f_{1}$ values from coarse mesh FEA and Bowie [1973] for the SECP specimen

Table 3.5: Comparison of $f_{2}$ values from coarse mesh FEA and Bowie [1973] for the SECP specimen

Table 3.6: Comparison of $t$ values from coarse mesh FEA and Wang [2001] for the SECP specimen

Table 3.7: $f_{l}$ values for CCP specimen from comprehensive FEA

Table 3.8: $f_{2}$ values for CCP specimen from comprehensive FEA 51

Table 3.9: $t$ values for CCP specimen from comprehensive FEA 51

Table 3.10: $f_{l}$ values for SECP specimen from comprehensive FEA 52

Table 3.11: $f_{2}$ values for SECP specimen from comprehensive FEA

Table 3.12: $t$ values for SECP specimen from comprehensive FEA

\section{Chapter 4: Crack-Tip Plastic Zone Analysis Under Static Mixed-Mode} Conditions

Table 4.1: Data for mapping the normalized plastic zone $r_{p}(\theta) / h$ for $\phi=0^{\circ} \quad 85$

Table 4.2: Data for mapping the normalized plastic zone $r_{p}(\theta) / h$ for $\phi=10^{\circ} \quad 86$

Table 4.3 Data for mapping the normalized plastic zone $r_{p}(\theta) / h$ for $\phi=20^{\circ}$ 
Table 4.4: Data for mapping the normalized plastic zone $r_{p}(\theta) / h$ for $\phi=30^{\circ} \quad 88$

Table 4.5: Data for mapping the normalized plastic zone $r_{p}(\theta) / h$ for $\phi=40^{\circ} \quad 89$

Table 4.6: Data for mapping the normalized plastic zone $r_{p}(\theta) / h$ for $\phi=50^{\circ} \quad 90$

Table 4.7: Data for mapping the normalized plastic zone $r_{p}(\theta) / h$ for $\phi=60^{\circ} \quad 91$

Table 4.8: Data for mapping the normalized plastic zone $r_{p}(\theta) / h$ for $\phi=70^{\circ} \quad 92$

Table 4.9: Data for mapping the normalized plastic zone $r_{p}(\theta) / h$ for $\phi=80^{\circ} \quad 93$

Table 4.10: Data for mapping the normalized plastic zone $r_{p}(\theta) / h$ for $\phi=90^{\circ} \quad 94$

Table 4.11: Table of maximum plastic zone size for varying mode II to mode I SIF ratios and normalized $T$-stress values

Chapter 5: Crack-Tip Plastic Zone Analysis Under Cyclic Mixed-Mode Conditions

Table 5.1: Table of T-factors for varying mode II to mode I SIF ratios and normalized $\mathrm{T}$-stress values

Table 5.2: Test details for Gao et al's [1985] bi-axially loaded CCP specimen

Table 5.3: SIF's and T-stress results for Gao et al's [1985] specimen

Table 5.4: Comparison of SIF and T-stress results with that of Gao et al [1985]

Table 5.5: Normalized cyclic plastic zone size results neglecting T-stress for Tests 1-5 of Gao et al [1985]

Table 5.6: Normalized cyclic plastic zone size results considering $T$-stress for Tests 1-5 of Gao et al [1985]

Table 5.7: Maximum normalized plastic zone results for Tests 1-5 of Gao et al [1985] 
Table A1: Evaluation of the plus-minus-effect on the core $z_{c}$ for $\phi=20^{\circ}$

Table A2: Evaluation of the plus-minus-effect on the core $z_{c}$ for $\phi=60^{\circ}$

Appendix B: The Plastic Zone Discontinuity

Table B1: Evaluation of the \pm -effect on the core $z_{-180}$ for $\theta=-180^{\circ}$ and Poisson's ratio 0.3

Table B2: Evaluation of the \pm -effect on the core $z_{180}$ for $\theta=180^{\circ}$ and Poisson's ratio 0.3 


\section{List of Figures}

Chapter 1: Introduction

Figure 1.1: $\quad$ General (a) CCP and (b) SECP specimens 6

Chapter 2: $\quad$ Background and Literature Review

Figure 2.1: The three basic modes of fracture 26

Figure 2.2 Mixed mode I and mode II fracture 27

Figure 2.3 Definition of the coordinate axis around the crack-tip 28

Figure 2.4 Pure mode I plastic zone map under plane strain loading 29

Figure 2.5 Typical fatigue crack growth behavior in metals 30

Figure 2.6 Modified 8-node quadrilateral element 31

Figure 2.7 Degeneration of an 8-node quadrilateral element into a triangle at the crack-tip $\quad 32$

Figure 2.8 Arbitrary contour around the crack-tip 33

Figure 2.9 Closed contour around the crack-tip 34

Figure 2.10 Generalized (a) CCP and (b) SECP specimen geometry 35

Chapter 3: Stress Intensity Factor and T-Stress Solutions for Mixed-

Mode Test Specimens Subjected to Remote Tension Loading $\quad 36$

Figure 3.1: $\quad$ Center cracked plate (CCP) specimen 53

Figure 3.2 Single-edge cracked plate (SECP) specimen $\quad 54$

Figure 3.3 Boundary and loading conditions applied to (a) CCP and (b) SECP specimens for FEA $\quad 55$

Figure 3.4 CCP FEA mesh magnified $4 \mathrm{x}$ to show sub-mesh 56

Figure 3.5 CCP FEA refined sub-mesh (Magnification 125x) 56

Figure 3.6 SECP FEA mesh magnified 5x to show sub-mesh 57 
Figure 3.7 SECP FEA refined sub-mesh (Magnification 40x)

Figure 3.8 Typical mesh of a CCP specimen with $a / W=0.4$ oriented at (a) $\theta=0^{\circ}$ and (b) $\theta=15^{\circ}$

Figure 3.9 Typical mesh of a SECP specimen with $a / W=0.4$ oriented at (a) $\theta=0^{\circ}$ and (b) $\theta=15^{\circ}$

Figure 3.10 Normalized mode I SIF as a function of (a) $a / W$ and (b) $\theta$ for the CCP specimen

Figure 3.11 Normalized mode II SIF as a function of (a) $a / W$ and (b) $\theta$ for the CCP specimen

Figure 3.12 Normalized T-stress as a function of $a / W$ for the CCP specimen

Figure 3.13 Normalized T-stress as a function of $\theta$ for the CCP specimen

Figure 3.14 Normalized mode I SIF as a function of (a) $a / W$ and (b) $\theta$ for the SECP specimen

Figure 3.15 Normalized mode II SIF as a function of (a) $a W$ (b) $\theta$ for the SECP specimen

Figure 3.16 Normalized T-stress as a function of $a / W$ for the SECP specimen

Figure 3.17 Normalized T-stress as a function of $\theta$ for the SECP specimen

\section{Chapter 4: $\quad$ Crack-Tip Plastic Zone Analysis Under Static Mixed-Mode} Conditions

Figure 4.1a-i: $\quad$ Plastic zone maps for $\phi=0^{\circ}$

Figure 4.2a-i: Plastic zone maps for $\phi=10^{\circ}$

Figure 4.3a-i: Plastic zone maps for $\phi=20^{\circ}$

Figure 4.4a-i: Plastic zone maps for $\phi=30^{\circ}$

Figure 4.5a-i: Plastic zone maps for $\phi=40^{\circ}$

Figure 4.6a-i: Plastic zone maps for $\phi=50^{\circ}$

Figure 4.7a-i: Plastic zone maps for $\phi=60^{\circ}$ 
Figure 4.8a-i: Plastic zone maps for $\phi=70^{\circ}$

$\begin{array}{lll}\text { Figure 4.9a-i: } & \text { Plastic zone maps for } \phi=80^{\circ} & 120\end{array}$

Figure 4.10a-i: Plastic zone maps for $\phi=90^{\circ}-123$

Figure 4.11a-j Graphs of maximum plastic zone size as a function of the normalized $T$-stress for various phase angles

Figure 4.12 Combined graphs of maximum plastic zone size as a function of the normalized T-stress $\left(-1 \leq t_{y} \leq 1\right)$ for various phase angles

Figure 4.13 Combined graphs of maximum plastic zone size as a function of the normalized T-stress $\left(-0.75 \leq t_{y} \leq 0.75\right)$ for various phase angles

Figure 4.14a-e Graphs of maximum plastic zone size as a function of phase angle for various normalized cyclic $T$-stress values

Chapter 5: Crack-Tip Plastic Zone Analysis Under Cyclic MixedMode Conditions

Figure 5.1a-j: Graphs of T-factor as a function of the normalized cyclic Tstress for various phase angles

Figure 5.2 Combined graphs of $\mathrm{T}$-factor as a function of the normalized cyclic $T$-stress for various phase angles

Figure 5.3a-e Graphs of $\mathrm{T}$-factor as a function of phase angle for various normalized cyclic $T$-stress values

Figure 5.4 Bi-axially loaded CCP specimen that was used by Gao et al [1985]

Figure 5.5a Principle of superposition as applied to Gao et al's [1985] biaxially loaded CCP specimen

Figure 5.5b Equivalent models of the transversely loaded component of thesuperposition principle as applied to the bi-axially loaded $\mathrm{CCP}$ in Figure 5.2a 
Figure 5.6a-e Normalized cyclic plastic zone maps calculated for Tests 1-5 of Gao et al's [1985] bi-axially loaded CCP specimen

Figure 5.7 Comparison of maximum cyclic plastic zone size with and without T-stress for Tests 1-5 of Gao et al's [1985] CCP specimen 


\section{List of Appendices}

Appendix A: The Crack-tip Plastic Zone Approximation

173

Tables

180

Appendix B: The Plastic Zone Discontinuity

182

Tables

190

Appendix C: Trigonometric Identities 


\section{Nomenclature}

\section{Acronyms}

$\begin{array}{ll}\text { CCP } & \text { Center Cracked Plate } \\ \text { FEA } & \text { Finite Element Analysis } \\ \text { GUI } & \text { Graphical User Interface } \\ \text { PZS } & \text { Plastic Zone Size } \\ \text { SECP } & \text { Single-Edge Cracked Plate } \\ \text { SIF } & \text { Stress Intensity Factor } \\ \text { SSY } & \text { Small Scale Yielding }\end{array}$

\section{Symbols}

$\begin{array}{ll}I, I I, I I I & \text { Modes of Fracture } \\ a u x & \text { Auxiliary } \\ \text { eff } & \text { Effective } \\ \text { int } & \text { Interaction } \\ \text { Max, min } & \text { Maximum, Minimum } \\ C, n & \text { Experimentally Determined Material Constants used in Paris and } \\ & \text { Erdogan's Power-Law Relationship } \\ (x, y) & \text { Cartesian Coordinates } \\ (r, \theta) & \text { Polar Coordinates } \\ d a / d N & \text { Crack Growth (da) per cycle }(d N) \\ {[\mathrm{a}],[\mathrm{b}],[\mathrm{c}]} & \text { Trigonometric Functions that form the Basis of The Plastic Zone } \\ & \text { Expression } \\ A & \text { Area } \\ a & \text { Crack Length }\end{array}$




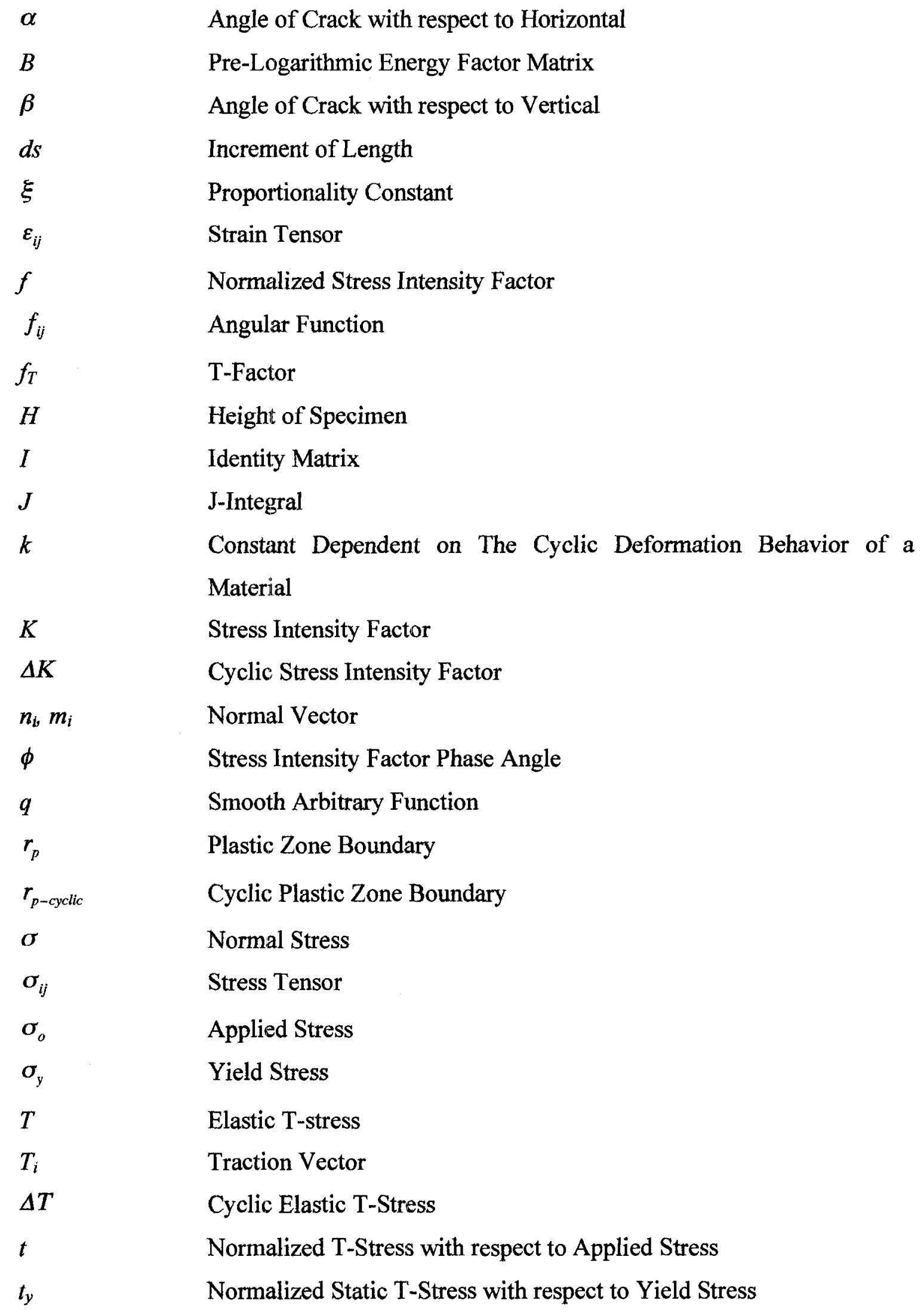

$n_{i}, m_{i}$

$r_{p-c y c l i c} \quad$ Cyclic Plastic Zone Boundary

$\Delta T \quad$ Cyclic Elastic T-Stress

Angle of Crack with respect to Horizontal

Pre-Logarithmic Energy Factor Matrix

Angle of Crack with respect to Vertical

Increment of Length

Proportionality Constant

Strain Tensor

Normalized Stress Intensity Factor

Angular Function

T-Factor

Height of Specimen

Identity Matrix

J-Integral

Constant Dependent on The Cyclic Deformation Behavior of a

Material

Stress Intensity Factor

Cyclic Stress Intensity Factor

$\phi$

Normal Vector

Stress Intensity Factor Phase Angle

Smooth Arbitrary Function

$r_{p} \quad$ Plastic Zone Boundary

$\sigma \quad$ Normal Stress

$\sigma_{i j} \quad$ Stress Tensor

$\sigma_{o} \quad$ Applied Stress

$\sigma_{\mathrm{y}} \quad$ Yield Stress

Elastic T-stress

$T_{i}$ Traction Vector

$t$

Normalized T-Stress with respect to Applied Stress

Normalized Static T-Stress with respect to Yield Stress 


$\begin{array}{ll}\Delta t_{y} & \text { Normalized Cyclic T-Stress with respect to Yield Stress } \\ \tau & \text { Shear Stress } \\ u_{i} & \text { Displacement Vector } \\ v & \text { Poisson's Ratio } \\ \gamma_{f} & \text { Material Fracture Ductility } \\ w & \text { Strain Energy Density } \\ W & \text { Specimen Width } \\ W_{p} & \text { Weighting Factor } \\ \Gamma & \text { Arbitrary Contour that Bgins and Ends at the bottom and Top Crack } \\ & \text { Surface Respectively }\end{array}$




\section{Chapter 1}

\section{Introduction}

Single-parameter fracture mechanics, as the name implies, refers to the use of a single parameter to characterize fracture. Due to the inherent simplicity in using a single parameter, single-parameter fracture mechanics has become a well-accepted and wellestablished means of studying and analyzing fatigue and fracture in engineering components. Single-parameter fracture mechanics is used to assess fatigue and fracture in a variety of industries including, but not limited to, the aviation, automotive, and naval industries. One of the most commonly used parameters in single-parameter fracture mechanics is the stress intensity factor (SIF). The SIF has been used to characterize material fracture toughness using the critical mode I SIF $K_{I C}$, as well as assess fatigue crack propagation rates using the cyclic SIF $\Delta K$ [Anderson, 2005].

However, it has been established that the use of single-parameter fracture mechanics is valid only in cases where the small-scale yielding (SSY) approximation holds, that is, in those cases where the crack-tip yield zone is significantly smaller than all other dimensions for a given specimen including the crack length [Anderson, 2005]. Many researchers, in the course of their work, have observed deviations between experimental results and those predicted through the use of single-parameter fracture mechanics [Du \& Hancock, 1991; Selvarathinam \& Goree, 1998; Tong, 2002]. Such deviations imply that the SSY approximation does not always hold and therefore singleparameter fracture mechanics cannot be applied to every fracture situation [Larsson \& Carlsson, 1973; Rice, 1974; Du et al, 1991]. Here we consider the situation where the size of the crack-tip plastic zone is still small enough to be characterized by the SIF, but so large that the SIF needs to be combined with another parameter to more accurately characterize the plastic zone.

The limitation of the SSY approximation has led to the development of twoparameter fracture mechanics, i.e., the use of two parameters to characterize fracture. The 
most common pair of terms used in two-parameter fracture mechanics is the SIF coupled with the T-stress, where the T-stress is a constant stress that acts parallel to the crack front [Larsson \& Carlsson, 1973; Rice, 1974]. The T-stress together with the SIF characterizes the stress field around the crack-tip beyond the SSY approximation where the SIF alone is not enough [Williams, 1957]. Therefore, by taking into consideration the combined effect of the SIF and the T-stress when characterizing fracture, a more accurate analysis can be conducted.

Most fracture mechanics analysis has been focused on pure mode loading conditions (i.e. opening, sliding, or tearing modes), however, in reality mixed-mode situations are just as likely to occur, for example, small cracks almost always initiate under a mixed-mode condition, and not all parts are loaded simply; many parts experience complex loading, such as moment or bi-axial loading, which produce a mixed-mode condition at the crack-tip. As such, it is important to expand the scope of fracture mechanics to include mixed-mode analysis, which for the purpose of this thesis results in mixed-mode two-parameter fracture mechanics analysis.

Center cracked plate (CCP) and single-edge cracked plate (SECP) specimens are two of the most commonly used test specimens in mixed-mode fatigue and fracture analysis (Fig. 1.1). Currently, for longitudinally loaded CCP and SECP specimens, there is well-established data concerning the mixed mode I and mode II SIF's for cracks of varying lengths and orientations with respect to the horizontal [Wilson, 1971; Bowie, 1973; Kitagawa \& Yuuki, 1977]. However, the T-stress data available for the longitudinally loaded CCP and SECP specimens are limited to a mode I loading condition [Kfouri, 1986; Sham, 1991; Wang, 2002]. Since, mixed-mode loading conditions could be prevalent in practice, SIF and T-stress data for the CCP and SECP specimens under mixed-mode loading conditions for different crack lengths and orientations need to be determined so that a comprehensive fracture analysis can be perfomed. 
The size of the crack-tip plastic zone has been determined to be a good indicator of constraint [Smith et al, 2001], material fracture resistance [Kang \& Beom, 2000] and fatigue crack growth rate [Gao et al, 1985; Wang \& Gao, 2007], therefore, the importance of the plastic zone cannot be emphasized enough. The crack-tip plastic zone has been studied for a number of pure mode and mixed-mode loading conditions influenced by $\mathrm{T}$ stress [Du \& Hancock, 1991; Ayatollahi et al, 2002; Subramanya et al, 2005]. However, a comprehensive study has not been performed on the plastic zone concerning the combined effect of different T-stress and mixed mode I and mode II SIF's under static and cyclic loading conditions. Performing a comprehensive mixed-mode two-parameter fracture mechanics analysis on the crack-tip plastic zone subjected to static and cyclic loading conditions will result in a better understanding of the crack-tip plastic zone as well as an improved comprehension of the combined effects of T-stress and mixed-mode loading on fracture and fatigue. Based on a comprehensive mixed-mode two-parameter fracture mechanics analysis, more accurate methods of predicting and analyzing fracture and fatigue can be developed.

\subsection{Objective of Thesis}

This thesis has three primary objectives. They are summarized as follows:

1. Determine the T-stress values for cracks of a CCP specimen (Fig. 1.1a) and a SECP specimen (Fig. 1.1b). The cracks in both the CCP and SECP specimens are varied in length from $0.1-0.8$ with respect to the specimen width. For each crack length, the crack is oriented at various angles between $0^{\circ}$ and $90^{\circ}$ with respect to the horizontal, thereby inducing a wide range of mixed-mode stress state. By analyzing crack orientations between $0^{\circ}$ and $90^{\circ}$ with respect to the horizontal, the solutions for all possible crack orientations will be addressed.

2. Develop an expression to model the crack-tip plastic zone under a general static mixed-mode loading condition influenced by $\mathrm{T}$-stress. Using the expression for the static plastic zone, the plastic zone is analyzed for varying combinations of mode II to mode I SIF ratios and T-stress. The static plastic zone expression is 
also proposed as a means of characterizing constraint and material fracture resistance.

3. Adapt the static plastic zone expression for cyclic loading, and use it to develop a fatigue crack propagation model that takes into consideration the T-stress effect on mixed-mode loading. The fatigue crack propagation model is then studied for varying combinations of mode II to mode I SIF ratios and T-stress.

\subsection{Outline of Thesis}

In addition to the current introductory chapter, this thesis is comprised of five more chapters and three appendices.

Chapter 2 provides a review of literature to-date as well as the necessary background theory; both of which are required to understand the development, current relevance, and necessity of the data and expressions derived in Chapters 3, 4, and 5.

Chapter 3 provides a description of the finite element analysis (FEA) conducted on CCP and SECP specimens under a uniform longitudinal load for varying crack orientations thereby inducing a mixed mode loading condition. The SIF and T-stress data obtained from the FEA is then analyzed to determine the significance and trends of the Tstress for different loading conditions.

Chapter 4 demonstrates the derivation of a mathematical expression for the cracktip plastic zone under static loading conditions. The derivation of the plastic zone expression is so detailed and complex that the author has chosen to include them as appendices, where Appendix A provides the complete derivation of the static plastic zone model, Appendix B describes the development of an expression to investigate the nature of the plastic zone across the crack-front, and Appendix $\mathrm{C}$ provides a list of trigonometric functions used in the derivation of the expressions in Appendices A and B. Chapter 4 also provides the details of a study conducted to investigate the trends of the plastic zone and the maximum plastic zone size with varying SIF and T-stress. Furthermore, the trends 
determined for the plastic zone are used to characterize the material fracture resistance in terms of mixed mode I and mode II SIF's and T-stress.

Chapter 5 illustrates the adaptation of the static plastic zone expression derived in Chapter 4 into one for cyclic loading. The cyclic plastic zone model is then applied to develop a relation for the fatigue crack growth rate that accounts for the T-stress effect under mixed-mode loading. Furthermore, the influence of the T-stress on the crack propagation is studied to again determine any trends and points of interest.

Finally, Chapter 6 concludes this thesis by summarizing the key points of each chapter. 


$$
\text { 政 }
$$




\section{Chapter 2}

\section{Background and Literature Review}

This chapter provides an overview of the theory and principles that are the foundation for the rest of this thesis while focusing on the advances made to-date with respect to them. The theories and principles covered are those of fracture mechanics, crack propagation analysis, and the finite element method with emphasis on the importance of mixed-mode loading and the effect of T-stress on the analysis of fatigue crack propagation behaviour.

\subsection{Fracture Mechanics}

Fracture mechanics is characterized by three basic modes of loading described, with reference to Figure 2.1, as follows [Anderson, 2005]:

- Mode I

The mode I loading condition, also referred to as the opening mode, is where the principle load is applied normal to the crack plane such that the crack is cleaved open perpendicular to the crack plane.

- Mode II

The mode II loading condition, also known as the sliding mode, is where the principle load is applied parallel to the crack plane such that it results in in-plane shear, that is, the top and bottom crack faces slide across each other in opposite directions perpendicular to the leading edge of the crack.

- Mode III

The mode III loading condition, also know as the tearing mode, is where the principle load is again applied parallel to the crack plane, but this time the load is such that it results in out-of-plane shear, where the crack faces slide across each other in opposite directions parallel to the leading edge of the crack. 
Fracture mechanics has gone through a number of evolutions and has developed a variety of facets [Anderson, 2005]. The facet of fracture mechanics that is of interest for the purpose of this thesis is that of linear elastic fracture mechanics (LEFM), which has evolved from using a single parameter to using two independent parameters to characterize the stress state at and around a crack-tip. The evolution of two-parameter fracture mechanics has resulted in more accurate methods for determining the service life of parts with varying specimen geometries, crack orientations, and loading conditions

The scope of LEFM has broadened from considering only the three individual pure modes of loading (Fig. 2.1) to taking into account mixed-mode loading conditions such as the mixed mode I and mode II loading condition (Fig. 2.2) which is the focus of this thesis.

\subsubsection{Single-Parameter Fracture Mechanics}

Conventional LEFM uses a single parameter to characterize the fracture process. The most common single parameter used to-date is the stress intensity factor (SIF), which describes the stress state at and around the tip of a crack in a linear elastic material [Anderson, 2005]. By applying the Williams series expansion [Williams, 1957], the SIF characterizes the crack-tip stress field for a two-dimensional crack (Fig. 2.3) as follows:

$\sigma_{i j}(\theta)=\frac{K_{I}}{\sqrt{2 \pi r}} f_{i j}^{I}(\theta)$

where $\sigma_{i j}$ represents the stress tensor components, $(r, \theta)$ are the polar coordinates centered at the crack-tip (Fig. 2.3), $K_{I}$ is the mode I SIF, and $f_{i j}^{I}$ is the mode I angular function.

From the Williams series expansion (Eqn 2.1), it can be observed that the SIF exhibits a $1 / \sqrt{r}$ singularity i.e., $\lim _{r \rightarrow 0} \sigma_{i j}(\theta)=\infty$, which results in a singularity-dominated zone where the SIF alone can be used to characterize the stress state around the crack-tip. 
The small-scale yielding (SSY) approximation, which is the basis of singleparameter fracture mechanics involving the SIF, assumes that the crack-tip plastic zone is significantly smaller than any relevant specimen dimension including the crack length, and, as such, is contained well inside the singularity-dominated zone. Therefore, all that is required to characterize the stress field outside the plastic zone but well within the specimen boundary is the SIF.

Assuming that a linear elastic material fails locally for some critical combination of stress and strain, then implicitly that material will fracture at some critical SIF called the fracture toughness. The fracture toughness is a material property that quantifies a material's resistance to fracture and for that reason, should the SIF induced as a result of the combined effect of the specimen geometry and applied force exceed the value of the material's fracture toughness then the specimen will fracture.

Single-parameter fracture mechanics involving the SIF provides a quick and simple means of evaluating the conditions under which a crack will grow and a given specimen will fail. However, observations of numerous researchers have shown that the results obtained from single-parameter fracture mechanics theory do not correspond with those obtained from experiments [Larsson \& Carlsson, 1973; Du \& Hancock, 1991; Qian \& Fatemi, 1996; Selvarathinam \& Goree, 1998; Tong, 2002; Yeh et al, 2006]. Additionally, it has been demonstrated that the range over which the SSY approximation is valid is considerably more limited than was proposed in earlier research works and that the SIF alone is insufficient to characterize the stress field around the crack-tip [Larsson \& Carlsson, 1973; Rice, 1974; Du et al, 1991]. The limitations of single-parameter fracture mechanics has resulted in the formulation of more accurate means of characterizing both the fracture process and the stress field around the crack-tip called two-parameter fracture mechanics. 


\subsubsection{Two-Parameter Fracture Mechanics}

As the name suggests, two-parameter fracture mechanics uses two independent parameters to characterize the stress field around a crack-tip and, therefore, also the fracture process. Similar to single-parameter fracture mechanics, the most common parameters used in two-parameter fracture mechanics are the SIF and the T-stress, where again the SIF describes the stress state at the crack-tip and the T-stress, a term first coined by Rice [1974], is used to denote the constant direct stress that acts parallel to the crack plane. Using the Williams series expansion [Williams, 1957], the stress field around the tip of a two-dimensional crack (Fig. 2.3) in a linear elastic material subjected to mode I loading can be expressed by the SIF and T-stress as follows:

$\sigma_{i j}(\theta)=\frac{K_{I}}{\sqrt{2 \pi r}} f_{i j}^{I}(\theta)+T \delta_{i 1} \delta_{1 j}$

where $\mathrm{T}$ is the elastic T-stress, and $\delta_{i 1} \delta_{1 j}$ is unity for $i=j=1$ and zero for $i, j \neq 1$.

In Equation 2.2, in spite of the presence of the T-stress, a singularity-dominated zone where the stress field can be characterized by the SIF alone still exists.

Larsson \& Carlsson [1973] and Rice [1974] have determined that including the Tstress, as a second parameter, to the SIF (Eqn 2.2) provides a suitable characterization for the crack-tip yield zone and therefore the stress field around the crack-tip as well. The conclusions obtained from Larsson \& Carlsson [1973] and Rice [1974] were further enforced by investigations conducted by several researchers including Tong [2002], and Seitl \& Knesl [2008] who have studied the consequence of T-stress on crack propagation and determined that the T-stress does affect the crack growth and needs to be taken into consideration when predicting crack growth so as to ensure better accuracy. Research by Smith et al [2001] has also demonstrated that the T-stress is a significant factor influencing fracture toughness and can therefore be used to better predict fracture. 
The T-stress has been used to characterize the effect of constraint on the triaxial stress state near the crack-tip. It has been observed from numerous research that under static loading conditions for a pure mode I case a positive $T$-stress strengthens the triaxial stress state at the crack-tip and results in an increase in constraint which promotes fracture, while negative $\mathrm{T}$-stress leads to the opposite i.e. the triaxial stress state at the crack-tip is weakened resulting in a decreased constraint which impedes fracture [Du \& Hancock, 1991; Ayatollahi et al, 1998; Tong, 2002; Wang et al, 2005]. Under cyclic loading conditions (fatigue) for a pure mode I case, an increase in constraint (positive Tstress) leads to a decrease in the crack growth rate, while a decrease in constraint (negative T-stress) leads to an increase in the crack growth rate [Hutar et al, 2004; Seitl \& Knesl, 2008].

Two-parameter fracture mechanics involving $\mathrm{T}$-stress is also referred to as constraint-based fracture mechanics due to the relationship that exists between the Tstress and the crack-tip triaxial constraint.

\subsubsection{Mixed-Mode Two-Parameter Fracture Mechanics}

The majority of fracture mechanics research is conducted for a pure mode I loading scenario (Fig. 2.1a), however, in reality a pure mode I scenario does not always occur and a mixed-mode scenario needs to be considered. A mixed-mode scenario is the consequence of the combined relative effect of crack orientation, specimen geometry, and loading conditions. For the purpose of this thesis, only mixed mode I and mode II loading conditions (Fig. 2.2) will be considered.

Expanding Equation 2.2 to include mixed mode I and mode II loading yields the following expression on the next page for the stress field surrounding the crack-tip (Fig. 2.3) [Williams, 1957]: 
$\sigma_{i j}=\frac{K_{I}}{\sqrt{2 \pi r}} f_{i j}^{I}(\theta)+\frac{K_{I I}}{\sqrt{2 \pi r}} f_{i j}^{I I}(\theta)+T \delta_{i 1} \delta_{1 j}$

where $K_{I I}$ is the mode II SIF, $f_{i j}^{I I}$ is the mode II angular function, and $i$ and $j=1,2$.

In two-parameter fracture mechanics involving T-stress, for a two-dimensional crack embedded in an isotropic linear elastic material subjected to mixed mode $I$ and mode II plain-strain loading conditions, Equation 2.3 expresses the stress field around the crack tip (Fig. 2.3) as follows [Anderson, 2005]:

$$
\begin{aligned}
& \sigma_{x x}=\frac{K_{I}}{\sqrt{2 \pi r}} \cos \frac{\theta}{2} \cdot\left[1-\sin \frac{\theta}{2} \cdot \sin \frac{3 \theta}{2}\right]-\frac{K_{I}}{\sqrt{2 \pi r}} \sin \frac{\theta}{2} \cdot\left[2+\cos \frac{\theta}{2} \cdot \cos \frac{3 \theta}{2}\right]+T \\
& \sigma_{y y}=\frac{K_{I}}{\sqrt{2 \pi r}} \cos \frac{\theta}{2} \cdot\left[1+\sin \frac{\theta}{2} \cdot \sin \frac{3 \theta}{2}\right]+\frac{K_{I I}}{\sqrt{2 \pi r}} \sin \frac{\theta}{2} \cdot \cos \frac{\theta}{2} \cdot \cos \frac{3 \theta}{2} \\
& \sigma_{z z}=v\left(\sigma_{x x}+\sigma_{y y}\right)=2 v\left\{\frac{K_{I}}{\sqrt{2 \pi r}} \cos \frac{\theta}{2}-\frac{K_{I I}}{\sqrt{2 \pi r}} \sin \frac{\theta}{2}\right\}+v T \\
& \tau_{x y} \neq \frac{K_{I}}{\sqrt{2 \pi r}} \sin \frac{\theta}{2} \cdot \cos \frac{\theta}{2} \cdot \cos \frac{3 \theta}{2}+\frac{K_{I I}}{\sqrt{2 \pi r}} \cos \frac{\theta}{2} \cdot\left[1-\sin \frac{\theta}{2} \cdot \sin \frac{3 \theta}{2}\right] \\
& \tau_{y z}, \tau_{z x}=0
\end{aligned}
$$

where, $(x, y)$ are the Cartesian coordinates originating at the crack tip (Fig. 2.3), and $v$ is Poisson's ratio. The elastic T-stress in Equation 2.4a induces a stress equivalent to $v T$ in the z-direction under plane strain loading conditions, which was dubbed the S-stress by Rice [1974]. $\sigma$ and $\tau$ are the normal and shear stress respectively with the subscripts denoting the direction of the stresses. 
By introducing T-stress as a second parameter in two-parameter fracture mechanics, the stress field around the crack-tip is much better approximated thereby allowing more accurate predictions for crack growth under static and cyclic loading to be formulated for mixed-mode conditions.

\subsection{The Plastic Zone under Static Loading}

In actual materials, the stresses are limited by the yield stress, which results in the formation of a plastic zone at the crack-tip. The plastic zone, also known as the yield zone and the damage zone, is defined as the region surrounding the crack-tip that has yielded as a result of a stress field that is induced by the applied specimen loading i.e., Equation 2.3. The plastic zone has been studied by a number of researchers for the purpose of determining its size so as to establish the validity of the SSY approximation [Larsson \& Carlsson, 1973; Rice, 1974]. In recent years, however, the plastic zone has been regarded as a measure of material resistance against fracture for static loading conditions where a larger plastic zone is indicative of a higher fracture resistance [Kang $\&$ Beom, 2000], while for fatigue loading condition, the plastic zone size is a measure of crack propagation where a larger plastic zone size results in a faster crack propagation rate [Gao et al, 1985; Park et al, 1996; Wang \& Gao, 2007]. The plastic zone has also been regarded as a means of characterizing constraint, where a high constraint lowers the influence of yielding and results in a smaller plastic zone [Smith et al, 2001].

One of the simplest methods of evaluating the plastic zone is Irwin's model [Anderson, 2005], which defines the plastic zone under pure mode I plane-strain loading conditions at $\theta=0^{\circ}$ as:

$r_{p}\left(\theta=0^{o}\right)=\frac{1}{6 \pi}\left(\frac{K_{I}}{\sigma_{y}}\right)^{2}$

where $r_{p}$ is the plastic zone boundary centered at the crack-tip, and $\sigma_{y}$ is the material yield stress. 
Similar to what was done in this thesis, Equation 2.5 is a first-order estimate in that Irwin approximated the applied stress field by assuming a linear-elastic stress field that is unaffected by the presence of the induced plastic zone [Anderson, 2005].

More complex analyses of the plastic zone, such as that described by the following expression for a pure mode I plane-strain loading condition [Anderson, 2005]:

$$
r_{p}(\theta)=\frac{1}{4 \pi}\left(\frac{K_{I}}{\sigma_{y}}\right)^{2}\left((1-2 v)^{2}(1+\cos \theta)+\frac{3}{2} \sin ^{2} \theta\right)
$$

where the complete plastic zone can be mapped for $-180^{\circ} \leq \theta \leq 180^{\circ}$ (Fig. 2.4) have been developed by incorporating the stress field (Eqn 2.3) into the Von Mises yield criterion below [Shigley, 2003]:

$$
\left[\left(\sigma_{x x}-\sigma_{y y}\right)^{2}+\left(\sigma_{y y}-\sigma_{z z}\right)^{2}+\left(\sigma_{z z}-\sigma_{x x}\right)^{2}+6\left(\tau_{x y}^{2}+\tau_{y z}^{2}+\tau_{z x}^{2}\right)\right]=2 \sigma_{y}
$$

As with the models above, many of the plastic zone models developed thus far only consider the effect of mode I loading while few models have been developed to consider the effects of mixed mode I and mode II loading, such as that of Gao et al [1985] below, who also employed the Von Mises yield criterion:

$$
\begin{aligned}
r_{p}(\theta)=\frac{1}{2 \pi \sigma_{y}^{2}}\left[K_{I}^{2}\left\{\cos ^{2} \frac{\theta}{2}\left(3 \sin ^{2} \frac{\theta}{2}+(1-2 v)^{2}\right)\right\}+\right. & K_{I} K_{I I}\left\{\sin \theta\left(3 \cos \theta-(1-2 v)^{2}\right)\right\} \\
+ & \left.+K_{I I}^{2}\left\{3+\sin ^{2} \frac{\theta}{2}\left(-9 \cos ^{2} \frac{\theta}{2}+(1-2 v)^{2}\right)\right\}\right]
\end{aligned}
$$


Even more uncommon are mathematical models of the plastic zone that take into account the effect of T-stress. Gao et al [1985] recommended that, if taking the T-stress effect into account, the plastic zone could be solved for numerically. A numerical evaluation of the plastic zone would be an incredibly tedious and time-consuming process.

What has been done, however, are FEM analysis to investigate the effect of mixed-mode loading and T-stress on the plastic zone. The FEM analysis showed that the plastic zone shape and size are influenced greatly by the T-stress depending on its sign and magnitude [Larsson \& Carlsson, 1973; Kim et al, 2001; Ayatollahi et al, 2002; Subramanya, 2005].

Therefore, to determine the validity of the SSY approximation, the resistance to fracture, and/or predict the fatigue crack growth rate, the impact of T-stress must be taken into account. To-date, there have not been any comprehensive studies on the effect of Tstress under mixed mode I and mode II loading conditions.

Chapter 4 of this thesis provides a detailed description of the extensive analysis performed to determine the effect of T-stress on the size and shape of the plastic zone, as well as the influence of $\mathrm{T}$-stress in characterizing constraint and predicting a material's resistance to fracture.

\subsection{Crack Propagation Analysis using The Plastic Zone under Cyclic Loading}

Fatigue crack propagation analysis has been studied since 1956 when Wells proved that the failures in a number of the Comet jet aircrafts were the result of fatigue cracks reaching their critical size. In 1960, Paris et al developed theories on the application of fracture mechanics principles to fatigue crack growth. Paris et al's theories 
would culminate in Paris and Erdogan's discovery of the following power-law relationship for fatigue crack growth [Anderson, 2005]:

$\frac{d a}{d N}=C(\Delta K)^{n}$

where $d a / d N$ is the crack growth $(d a)$ per cycle $(d N), C$ and $n$ are experimentally determined material constants, and $\Delta K=K_{\max }-K_{\min }$.

Typical fatigue crack propagation behavior of metals as a function of the cyclic SIF $\Delta K$ can be divided into three regions (Fig. 2.5), where region I reveals a "fatiguethreshold" SIF $\Delta K_{t h}$ below which no crack propagation will occur, region II exhibits the fatigue crack propagation behavior that is insensitive to the R-ratio $\left(K_{\max } / K_{\min }\right)$ and can be modeled by the power law relationship (Eqn 2.9), and region III shows the accelerated fatigue crack propagation rate up to final failure at the critical SIF $\boldsymbol{K}_{c}$.

Driven by economics and the need to advance the field of fracture mechanics, a variety of crack propagation models have been developed to improve fatigue life predictions for structural and mechanical components. The majority of these models are based on Paris and Erdogan's power law relationship and only take into account the effect of the SIF. Some researchers have adopted the use of an effective SIF in the power law relationship where the effective SIF is a parameter that quantifies the combined effect of for example, the mixed mode I and mode II SIF's [Tanaka, 1974] or of mode I SIF and T-stress [Hutar et al, 2004]. Another parameter that has been determined ideal for characterizing fatigue crack propagation is the plastic zone size [Park et al, 1996], which has been made use of with promising results by Gao et al [1985] and Wang \& Gao [2007]. An important property of the plastic zone size is that it can incorporate the effects of the mode I and mode II SIF's as well as the T-stress as they all affect the plastic zone. 
Wang \& Gao [2007] related the mode I plastic zone size to the crack propagation rate using the following expression:

$\frac{d a}{d N} \propto\left(r_{p-c y c l i c \max }\right)^{n / 2}$

where $n$ is the exponent of the power law relationship.

Wang and Gao [2007] then applied the following expression relating the mode I SIF and T-stress to the plastic zone size:

$r_{p-c y c l i c \max } \propto(\Delta K)^{2}\left[1-\xi \frac{\Delta T}{2 \sigma_{y}}\right]$

where $\xi$ is a proportionality constant, and $\Delta T=T_{\max }-T_{\min }$.

to Equation 2.10 to yield:

$\frac{d a}{d N}=C(\Delta K)^{n}\left[1-\xi \frac{\Delta T}{2 \sigma_{y}}\right]^{n / 2}$

where $C$ is the coefficient of the power law relationship.

Equation 2.12 not only incorporates the T-stress effect, but also maintains the power law relationship.

Gao et al [1985] developed an expression that related the mixed mode I and mode II SIF's and the T-stress to the plastic zone size (Eqn 2.8), and then went on to relate the plastic zone size to the fatigue crack propagation rate using the following formula on the next page: 
$\frac{d a}{d N}=k \frac{r_{p \max }}{\gamma_{f}}$

where $k$ is a constant dependent on the cyclic deformation behavior of the material, $r_{p \max }$ is the maximum plastic zone size, and $\gamma_{f}$ is the material fracture ductility.

Mixed-mode loading and T-stress plays a significant role in fracture mechanics and yet their combined effect has not been incorporated into an expression to predict fatigue crack propagation behavior. This thesis will detail the derivation of an expression for the plastic zone size that incorporates the effect of mixed mode I and mode II loading conditions and the T-stress (Chapter 4), and then use it to predict fatigue crack growth behavior (Chapter 5).

\subsection{The Finite Element Method}

The finite element method (FEM) has established itself as a trusted and accurate means of evaluating fracture specimens and determining fracture mechanics parameters that include but are not limited to the J-integral, SIF's, and T-stress.

This section of the chapter aims to provide background theory on how FEM was used in Chapter 3 of this thesis to evaluate the J-integral and extract the SIF's and Tstress for plate specimens subjected to mixed mode I and mode II loading conditions.

\subsubsection{The Crack-Tip Stress Singularity}

As was observed earlier, the stress in close vicinity of the crack-tip varies as $1 / \sqrt{r}$ in an elastic material resulting in a stress singularity or an unbounded stress as $r \rightarrow 0$ i.e., at the crack-tip. The presence of a stress singularity in any FEM model makes it impossible to accurately represent the stress singularity using conventional isoparametric elements due to a number of reasons that include the corruption of results obtained at the nodes of elements neighboring the singularity, as well as the dependency of the stress 
singularity on the mesh density such that a denser mesh will produce a higher value of results [Huebner et al, 2001].

To address the issue of accurately representing a $1 / \sqrt{r}$ stress singularity in an FEM model, a modified 8-node quadrilateral element was created (Fig. 2.6) such that, referring to Figure 2.7, (a) one side of an 8-node quadrilateral (nodes 1, 2, and 5) was collapsed and fixed onto a single point thereby forming a triangle and (b) the mid-side nodes (node 6 and 8 ) of the sides connected to the collapsed point were moved to the quarter-point [Anderson, 2005]. The modified 8-node quadrilateral element, as displayed in Figure 2.6, not only possesses a singularity at the collapsed point, but also exhibits a $1 / \sqrt{r}$ variation over the two modified sides of the element and therefore provides an excellent means of accurately modeling the crack-tip singularity.

\subsubsection{Mixed-Mode Stress Intensity Factors and T-Stress}

ABAQUS/Standard (v. 6.6) [ABAQUS Theory Manual, 2006] uses an interaction integral method to extract the individual SIF's from the J-integral for a crack embedded in a linear elastic isotropic material subjected to mixed-mode loading conditions.

\section{The J-Integral}

First developed by Rice in 1968 to characterize non-linear material behavior, the $\mathrm{J}$-integral is a line integral that is evaluated along an arbitrary contour around a crack (Fig. 2.4) and used as an expression of non-linear energy release rates. The J-integral for a non-linear elastic body subject to 2 -dimensional deformation is give by [Anderson, 2005]:

$$
J=\int_{\Gamma}\left(w d y-T_{i} \frac{\partial u_{i}}{\partial x} d s\right)
$$

with, the strain energy density or stress work $w=\int_{o}^{\varepsilon_{i j}} \sigma_{i j} d \varepsilon_{i j}$ 
where $\Gamma$ is an arbitrary contour that begins and ends at the bottom and top crack surface respectively, $w$ is the strain energy per unit volume, $T_{i}$ is the component of the traction vector perpendicular to $\Gamma$ in the outside direction, $u_{i}$ is the displacement vector component, $d s$ is the increment of length along the contour $\Gamma$, and $\sigma_{i j}$ and $\varepsilon_{i j}$ are the stress and strain tensors respectively.

In 1986, Shih developed a generalized expression of the J-integral that allowed for numerical evaluation [Guo, 2005]. In Shih's expression, the J-integral is evaluated along a contour surrounding the crack that shrinks onto the crack-tip [Fig. 2.8]. Shih's Jintegral expression for quasi-static conditions is given as [Anderson, 2005]:

$$
J=\lim _{\Gamma_{0} \rightarrow 0} \int\left[w \delta_{\Gamma_{0}}-\sigma_{i j} \frac{\partial u_{j}}{\partial x_{1}}\right] n_{i} d \Gamma
$$

where $n_{i}$ is the unit vector normal to $\Gamma$, and $d \Gamma$ is the arc length.

Formulating Equation 2.16 for finite element analysis involves evaluating it along a closed contour (Fig. 2.9) and yields the following expression in the absence of crackface tractions [Anderson, 2005]:

$$
J=\int_{\Gamma^{*}}\left[\sigma_{i j} \frac{\partial u_{j}}{\partial x_{1}}-w \delta_{1 i}\right] q m_{i} d \Gamma
$$

where $\Gamma^{*}=\Gamma_{1}+\Gamma_{+}+\Gamma_{-}-\Gamma_{0}, \Gamma_{+}$and $\Gamma_{-}$are the upper and lower crack faces respectively, $q$ is the arbitrary but smooth function that is equal to unity on $\Gamma_{0}$ and zero on $\Gamma_{1}$, and $m_{i}$ is the outward normal on $\Gamma^{*}$ (Fig. 2.9). 
With some manipulation, Equation 2.17 reduces to the following 2-dimensional expression for a linear elastic material under quasi-static conditions without body forces, thermal strains, and crack-face tractions [Anderson, 2005]:

$J=\int_{A^{\cdot}}\left[\sigma_{i j} \frac{\partial u_{j}}{\partial x_{1}}-w \delta_{1 i}\right] \frac{\partial q}{\partial x_{i}} d A$

where $A^{*}$ is the area of the closed contour.

In its discretized form Equation 2.18 is given as [Anderson, 2005]:

$$
J=\sum_{A^{*}} \sum_{p=1}^{m}\left\{\left[\left(\sigma_{i j} \frac{\partial u_{j}}{\partial x_{1}}-w \delta_{1 i}\right) \frac{\partial q}{\partial x_{i}}\right] \operatorname{det}\left(\frac{\partial x_{j}}{\partial \xi_{k}}\right)\right\}_{p} W_{p}
$$

where $m$ is the total number of Gaussian points per element, $p$ is the Gaussian point at which the term is being evaluated, $\xi_{k}$ are the parametric coordinates for the element, and $W_{p}$ is the weighting factor specific for each Gaussian point.

\section{Extracting the Mode I and II Stress Intensity Factors}

For a crack under mixed mode I and mode II loading conditions, the SIF's are calculated as follows [ABAQUS Theory Manual, 2006]:

$$
K=4 \pi B J_{\text {int }}
$$

where the SIF matrix $K=\left[K_{1}, K_{I I}\right]^{T}, B$ is called the pre-logarithmic energy factor matrix, and the interaction integral $J_{\mathrm{int}}=\left[J_{\mathrm{int}}^{\prime}, J_{\mathrm{int}}^{I I}\right]^{T}$ is calculated as:

$$
J_{\text {int }}^{\alpha}=\lim _{\Gamma \rightarrow 0} \int_{\Gamma} n M^{\alpha} q d \Gamma
$$


with $M^{\alpha}=\sigma: \varepsilon_{a u x}^{\alpha} I-\sigma\left(\frac{\partial u}{\partial x}\right)_{a u x}^{\alpha}-\sigma_{a u x}^{\alpha} \frac{\partial u}{\partial x}$

where $\alpha$ represents the mode of fracture i.e. mode I or mode II, $I$ is the identity matrix, and the subscript aux refers to the auxiliary crack-tip field $J_{a u x}$ defined with SIF $k_{\alpha}$ as follows:

$J_{a u x}^{\alpha}=\frac{1}{8 \pi} k_{\alpha} B_{\alpha \alpha}^{-1} k_{\alpha}$

\section{Extracting the T-Stress}

The Williams series expansion of the stress field in the vicinity of a crack [Fig. 2.3] embedded in a linear elastic material subjected to mixed mode I and II plane strain loading conditions is given by:

$\sigma_{i j}=\frac{K_{I}}{\sqrt{2 \pi r}} f_{i j}^{I}(\theta)+\frac{K_{I I}}{\sqrt{2 \pi r}} f_{i j}^{I I}(\theta)+T \delta_{i l} \delta_{1 j}$

The T-stress is extracted through the application of an auxiliary solution of a line load with magnitude $f$ to the plane of crack propagation and along the crack line:

$$
\begin{aligned}
& \sigma_{11}^{L}=\frac{f}{\pi r} \cos ^{3} \theta \\
& \sigma_{22}^{L}=\frac{f}{\pi r} \cos \theta \cdot \sin ^{2} \theta \\
& \sigma_{33}^{L}=\frac{f}{\pi r} v \cos \theta \\
& \tau_{12}^{L}=\frac{f}{\pi r} \sin \theta \cdot \cos ^{2} \theta
\end{aligned}
$$


$\tau_{13}^{L}=\tau_{23}^{L}=0$

Applying the interaction integral in the same manner as was as applied in the extraction of the SIF's earlier results in the following solution for T-stress (neglecting temperature effects) under plane strain loading conditions [ABAQUS Theory Manual, 2006]:

$T=\frac{E}{\left(1-v^{2}\right)}\left[-\frac{I_{\text {int }}(s)}{f}\right]$

where the interaction integral $I_{\text {int }}$ is calculated as follows [ABAQUS Theory Manual, 2006]:

$I_{\text {int }}=\lim _{\Gamma \rightarrow 0} \int_{\Gamma} n M q d \Gamma$

with $M=\sigma: \varepsilon_{a u x}^{L} I-\sigma\left(\frac{\partial u}{\partial x}\right)_{a u x}^{L}-\sigma_{a u x}^{L} \frac{\partial u}{\partial x}$

ABAQUS/Standard (v6.6) evaluates the J-integral by first defining a domain in terms of rings of elements around the crack-tip [Fig. 2.7] and then choosing the term $q$ such that it has a magnitude of zero at the nodes on the outside of the domain and a magnitude of 1 at the inside of the domain [ABAQUS Theory Manual, 2006]. Once the Jintegral is evaluated, it is used to extract both the SIF's and T-stress.

\subsection{Mixed-Mode Test Specimens}

Two of the most popular testing specimens used to-date are the longitudinally loaded rectangular-shaped center cracked plate (CCP) and the single-edge cracked plate (SECP) specimens (Fig. 2.10) [Sherry et al, 1995; Fett, 1998]. The reason for the 
popularity of the CCP and SECP specimens is that they emulate the most common cracks found in practice.

The CCP specimen (Fig. 2.10a) has a width of $2 \mathrm{~W}$, and a height of $2 \mathrm{H}$, with a center-crack of length $2 a$ oriented at and angle of $\alpha$ with respect to the horizontal. The SECP specimen (Fig. 2.10b) has a width of $W$, and a height of $2 \mathrm{H}$, with an edge-crack of length $a$ oriented at an angle of $\alpha$ with respect to the horizontal. Both the CCP and SECP specimens have a uniform load $\sigma_{o}$ acting along their top and bottom edges in the longitudinal direction (Fig. 2.10).

Tests conducted on the longitudinally-loaded CCP and SECP specimens are primarily for mode I loading conditions i.e. $\alpha=0^{\circ}$ [Sherry et al, 1995; Fett, 1998; Wang, 2002]. In addition, there is well-established data available for CCP and SECP specimens uniformly loaded longitudinally, where a mixed-mode loading condition is induced by varying the crack angle $\alpha$ [Wilson, 1971; Bowie, 1973; Kitagawa \& Yuuki, 1977].

There is also well-established data available on the analysis of longitudinally loaded CCP and SECP specimens with respect to T-stress [Kfouri, 1986; Sham, 1991; Wang, 2002]. However, the available T-stress data concerns only that of a pure mode I loading condition; no T-stress data was found concerning mixed-mode loading conditions.

To comprehensively determine how the T-stress affects the fracture and fatigue of a longitudinally loaded CCP and SECP specimens, T-stress values must first be determined for various mixed-mode loading conditions by varying the crack orientation with respect to the horizontal, and to that end, a parametric study was conducted on the CCP and SECP specimens. A description of the study conducted by the author on the CCP and SECP specimens, as well as the accompanying SIF and T-stress results are provided in Chapter 3 of this thesis. 


\subsection{Concluding Remarks}

The theory and background provided in this chapter provides the basis upon which the rest of this thesis is founded.

Chapter 3 of this thesis will provide a description and results of a parametric study conducted on CCP and SECP specimens using FEM to determine the SIF and T-stress values for various crack geometries.

Chapter 4 will provide a description of the complex derivation and subsequent analysis of an expression to model the crack-tip plastic zone under static loading conditions. Chapter 4 also describes the application of the static crack-tip plastic zone size to characterize a material's resistance to fracture, thereby providing a relation for material fracture resistance that considers the influence of the T-stress under mixed-mode loading conditions.

Chapter 5 will describe how the static plastic model was adapted for cyclic loading conditions. The cyclic plastic zone is then used to develop a relation to better predict crack propagation rates by again taking into account the T-stress effect.

The author's intent is for the SIF and T-stress results of the parametric study described in Chapter 3 to be used in conjunction with the expressions derived in Chapters 4 and 5 to provide a relation for fracture resistance and to more accurately determine the crack growth rates for CCP and SECP specimens of a given linear-elastic material by considering the $\mathrm{T}$-stress effect under mixed-mode loading conditions. 
(a)

Mode I

(Opening) (b)

Mode II

(In-Plane Shear) (c)

Mode III

(Out-of-Plane Shear)

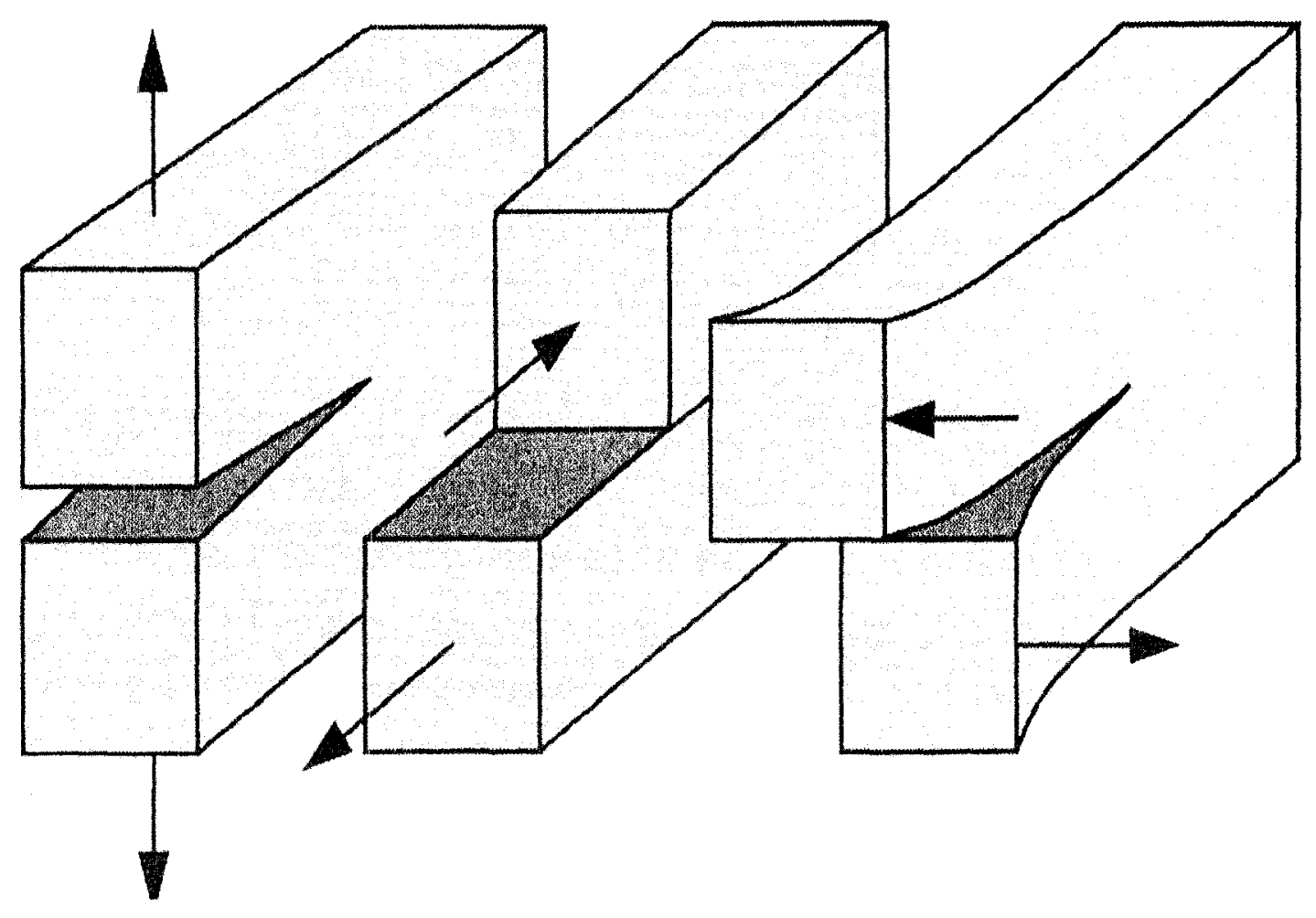

Figure 2.1: The three basic modes of fracture [Anderson, 2005] 


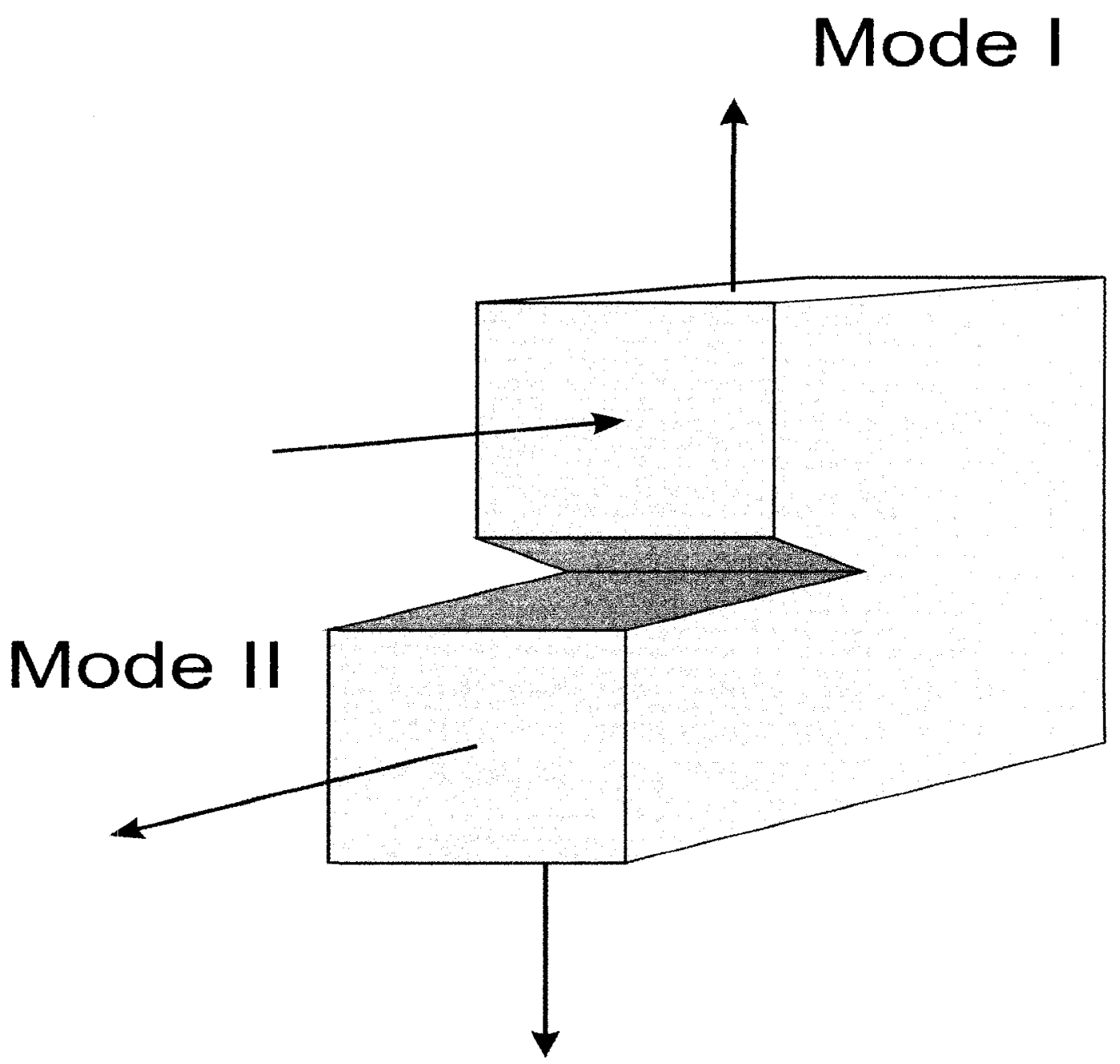

Figure 2.2: Mixed mode I and mode II fracture 


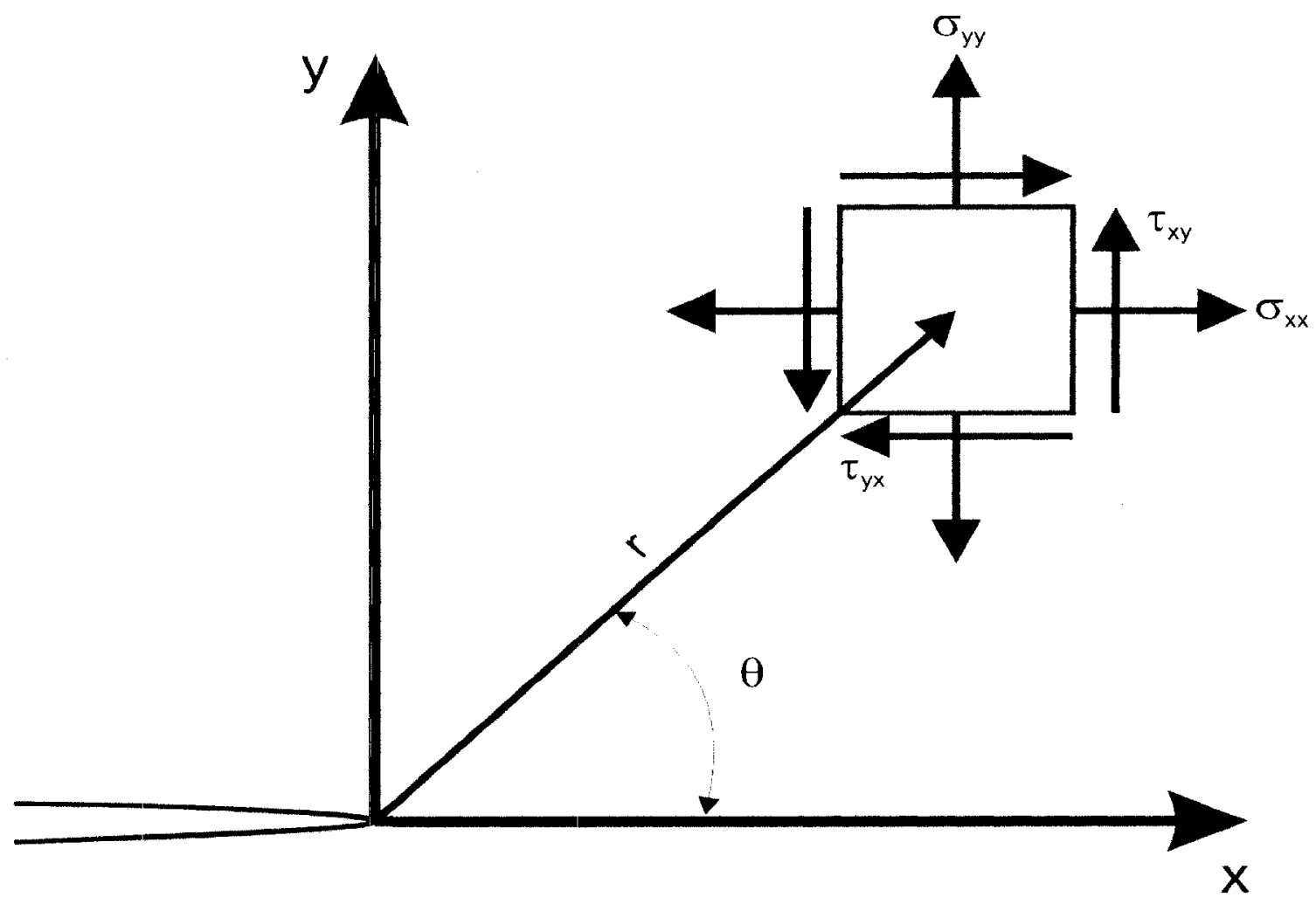

Figure 2.3: Definition of the coordinate axis around the crack-tip [Anderson, 2005] 


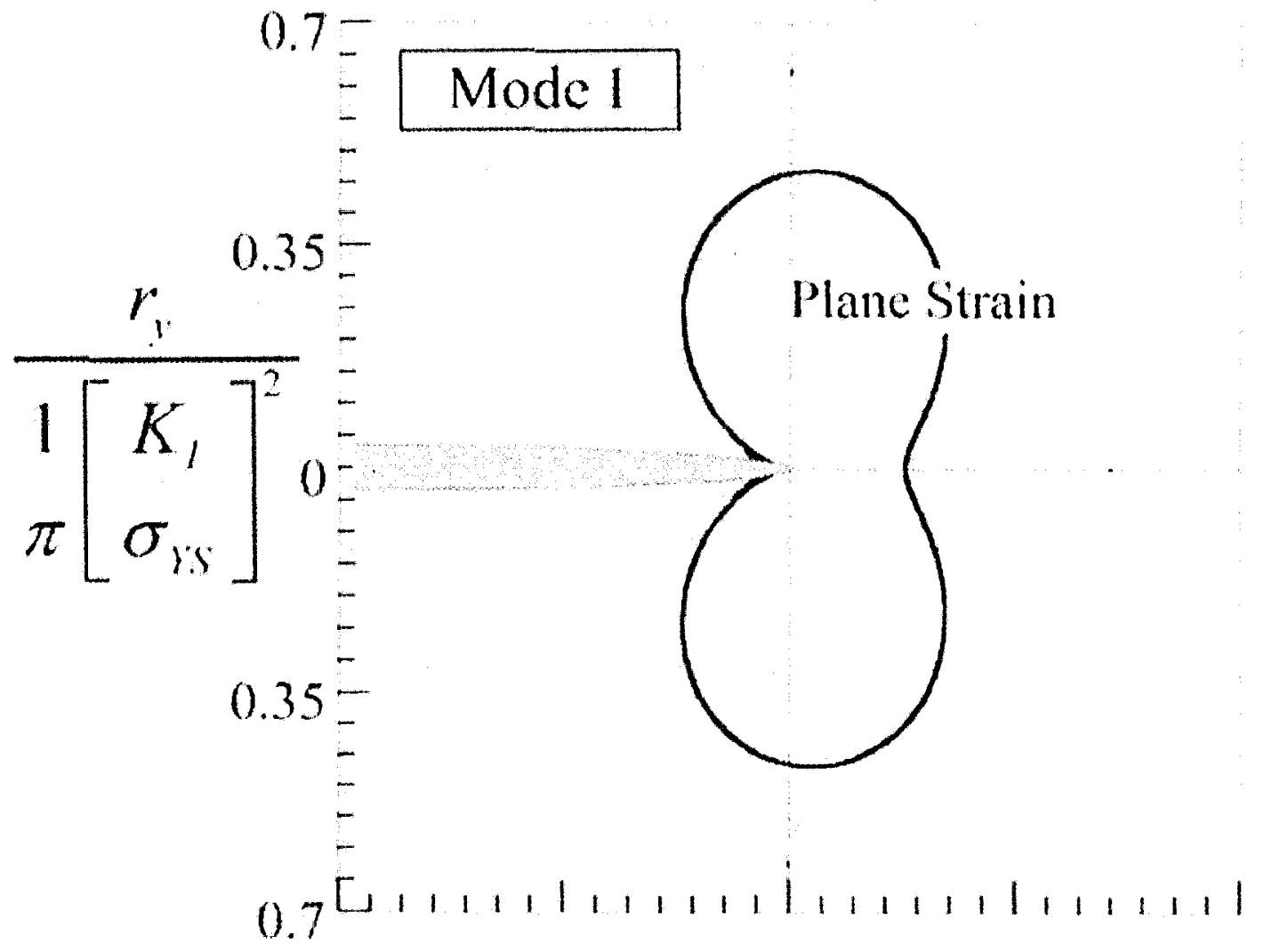

Figure 2.4: Pure mode I plastic zone map under plane strain loading [Anderson, 2005] 


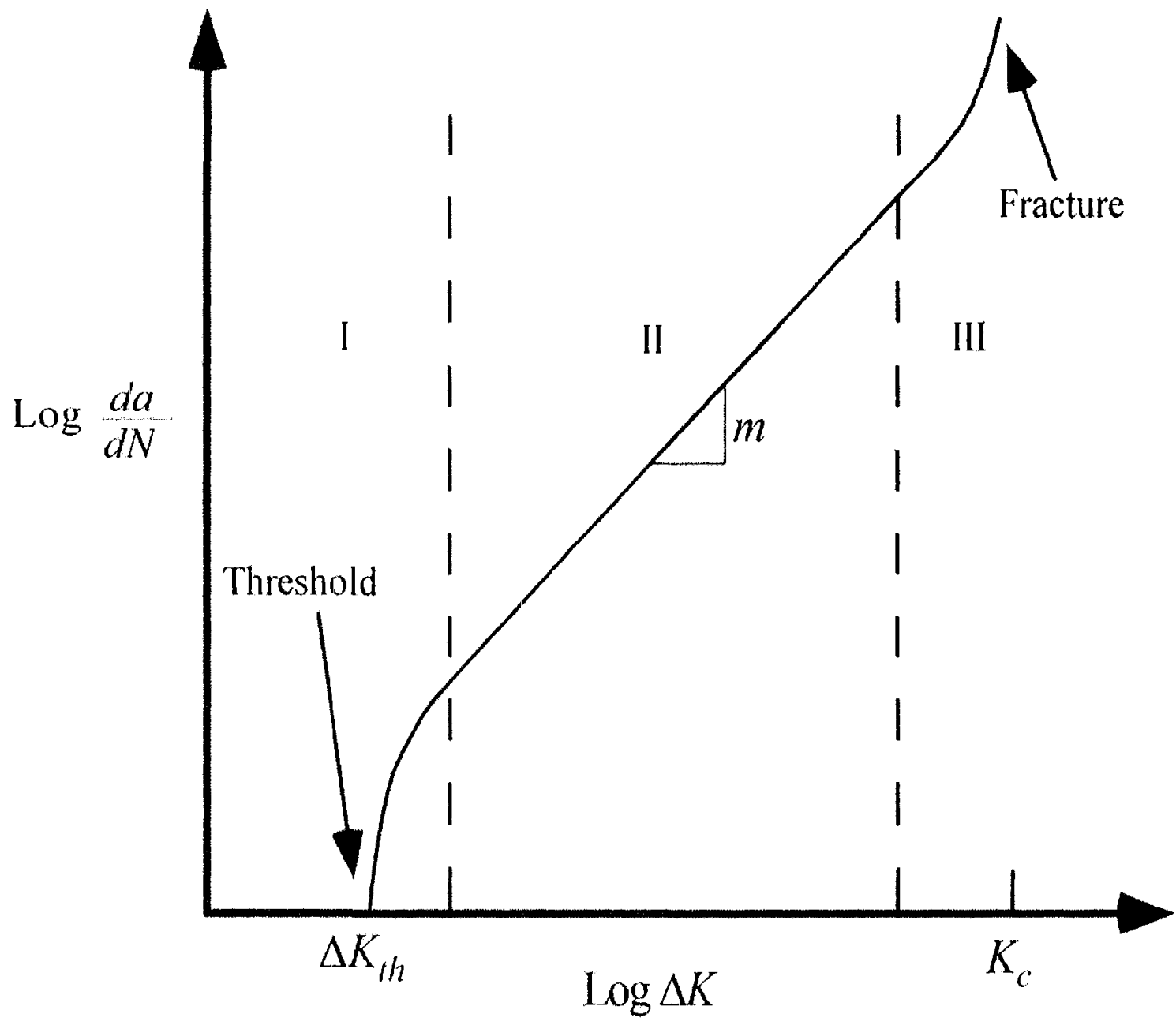

Figure 2.5: Typical fatigue crack growth behavior in metals [Anderson, 2005] 


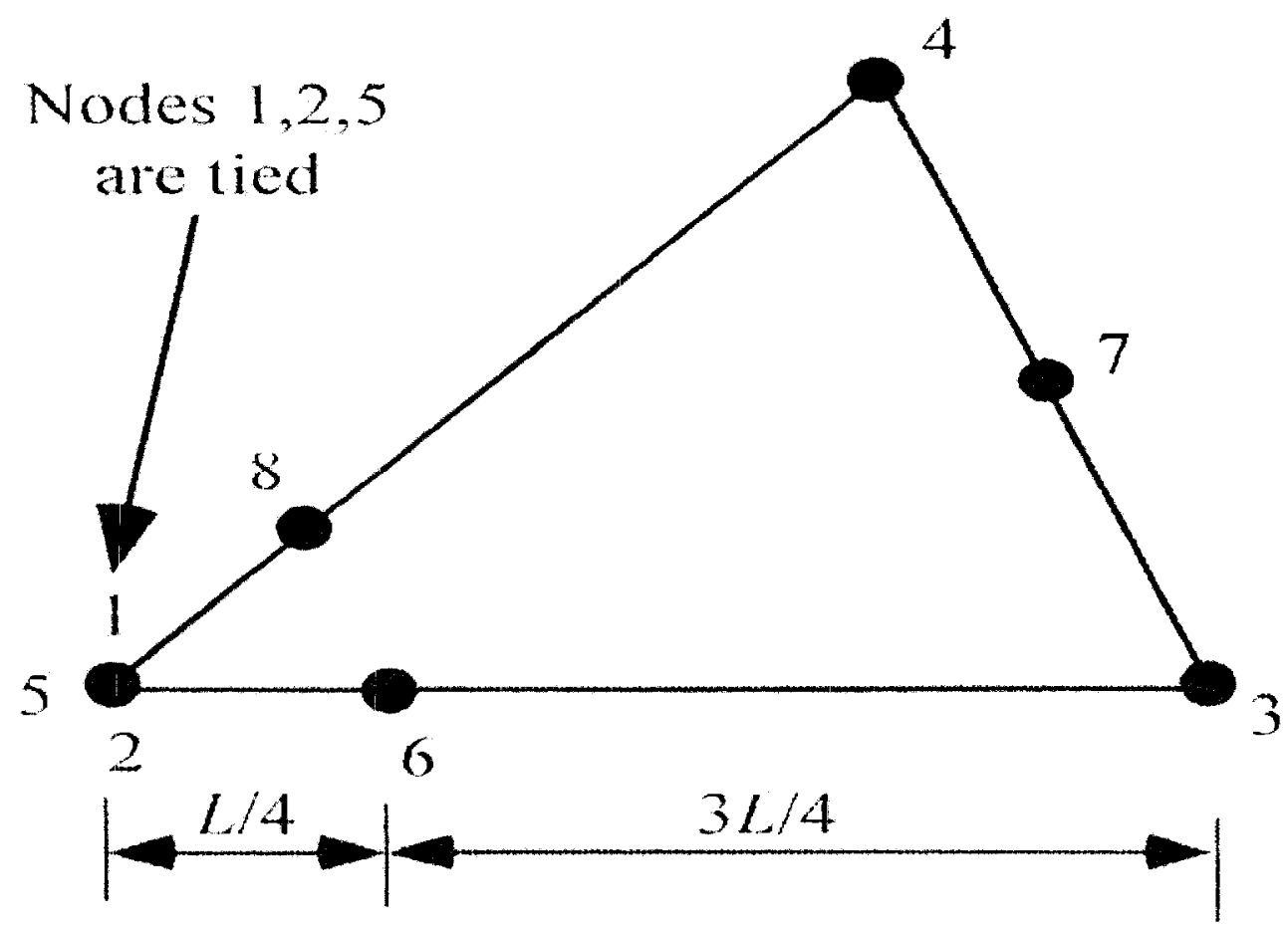

Figure 2.6: Modified 8-node quadrilateral element [Anderson, 2005] 


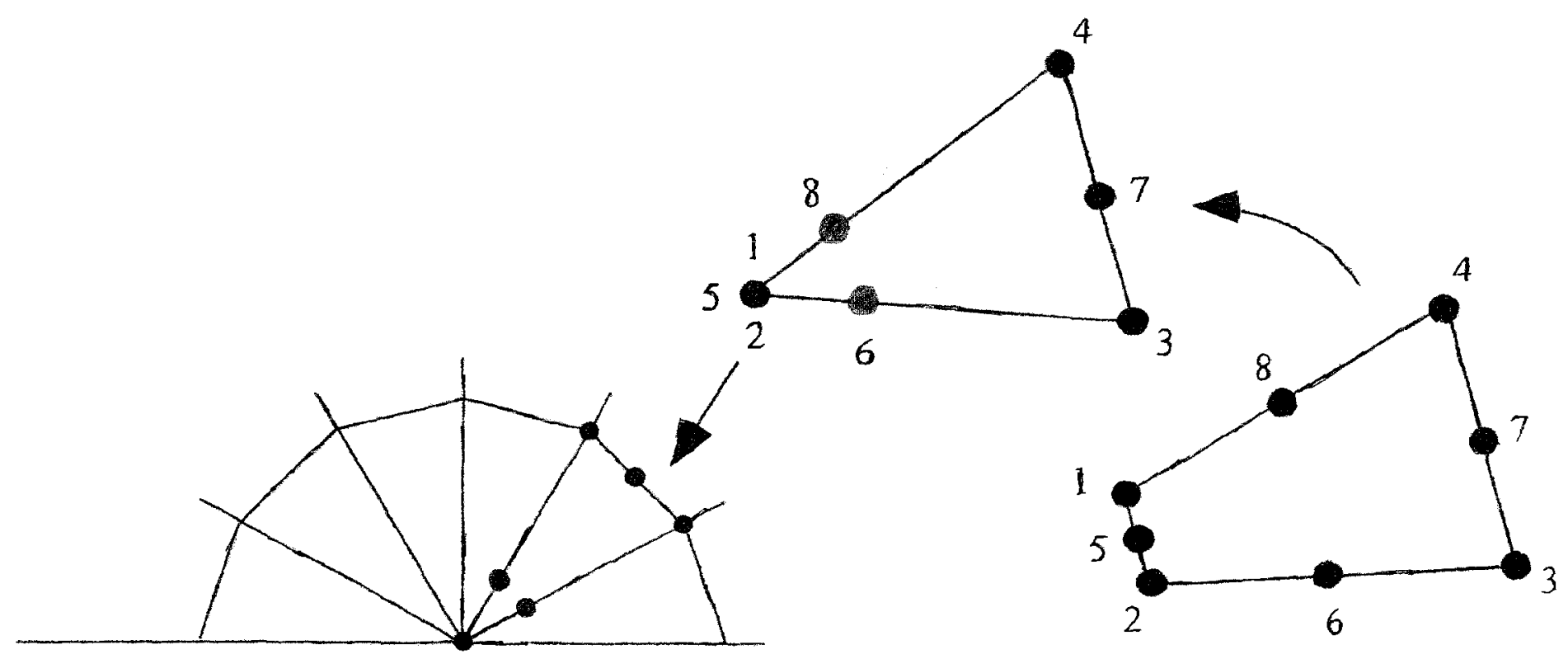

Figure 2.7: Degeneration of an 8-node quadrilateral element into a triangle at the crack-tip [Anderson, 2005] 


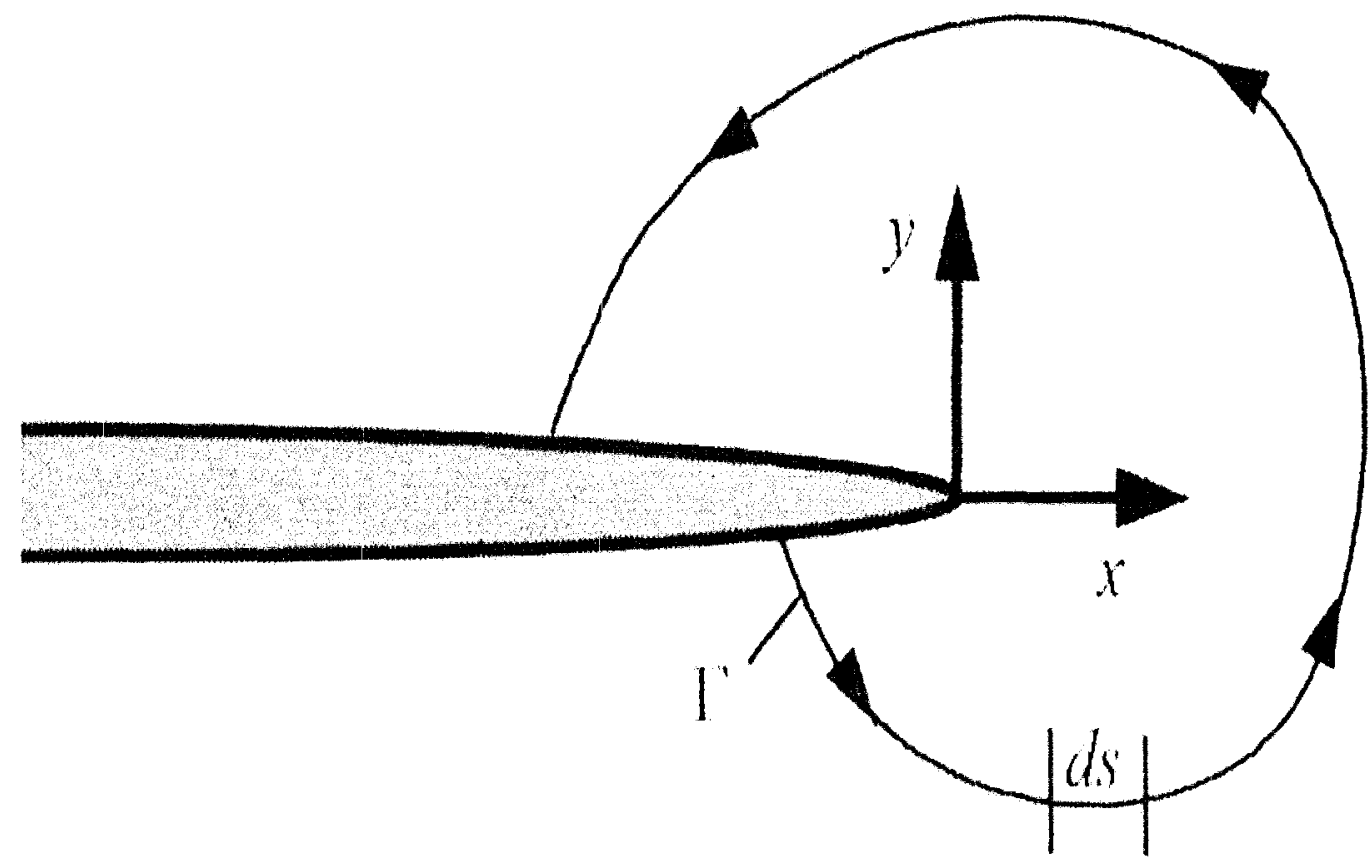

Figure 2.8: Arbitrary contour around the crack-tip [Anderson, 2005] 


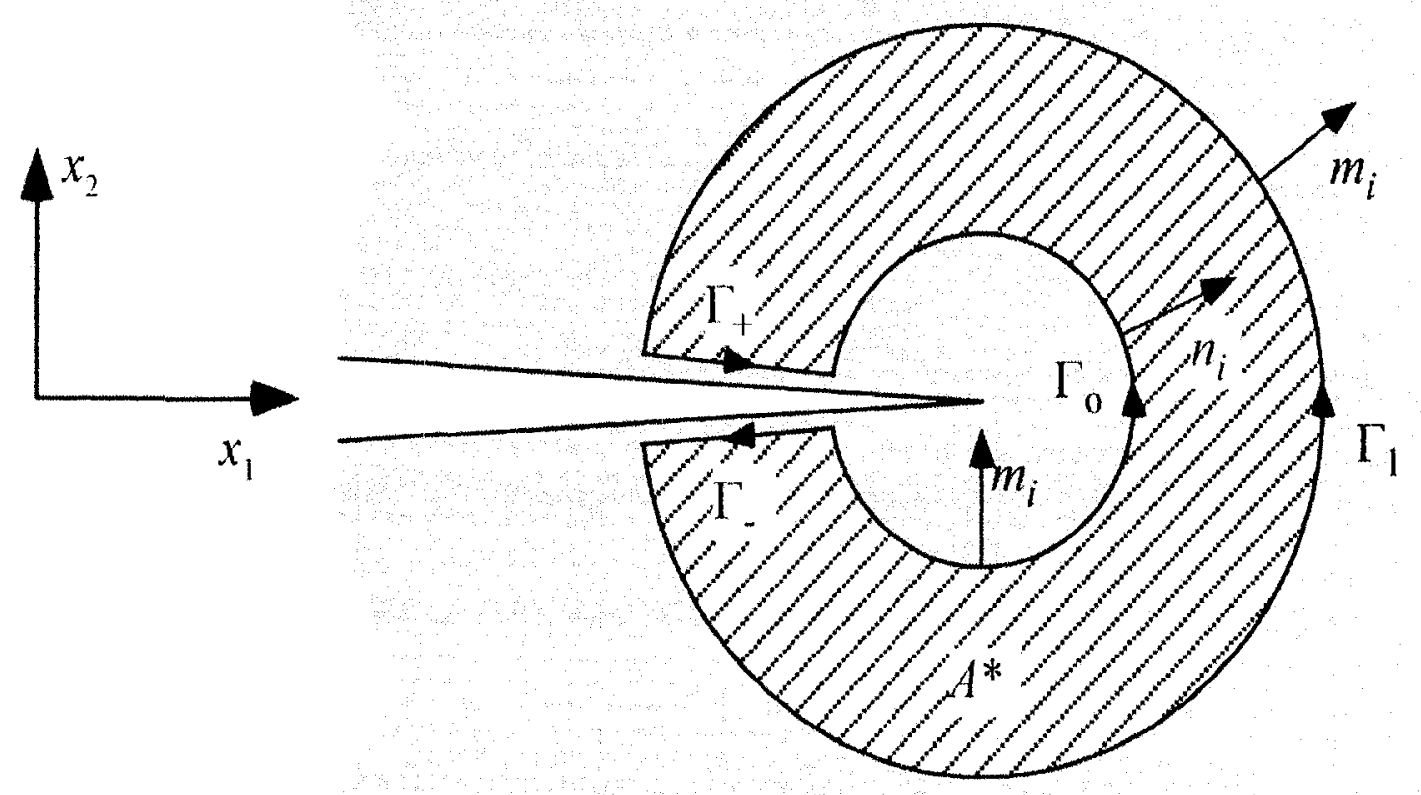

Figure 2.9: Closed contour around the crack-tip [Anderson, 2005] 


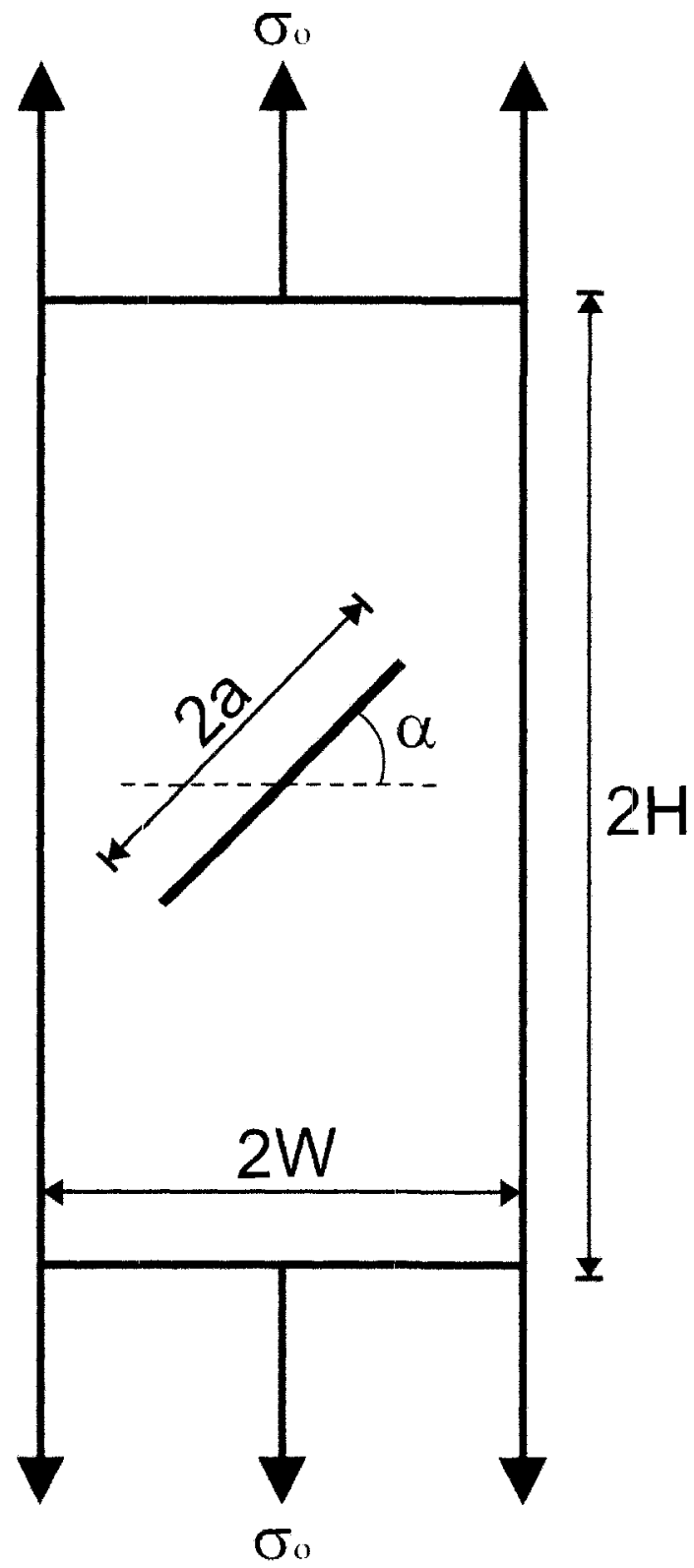

(a)

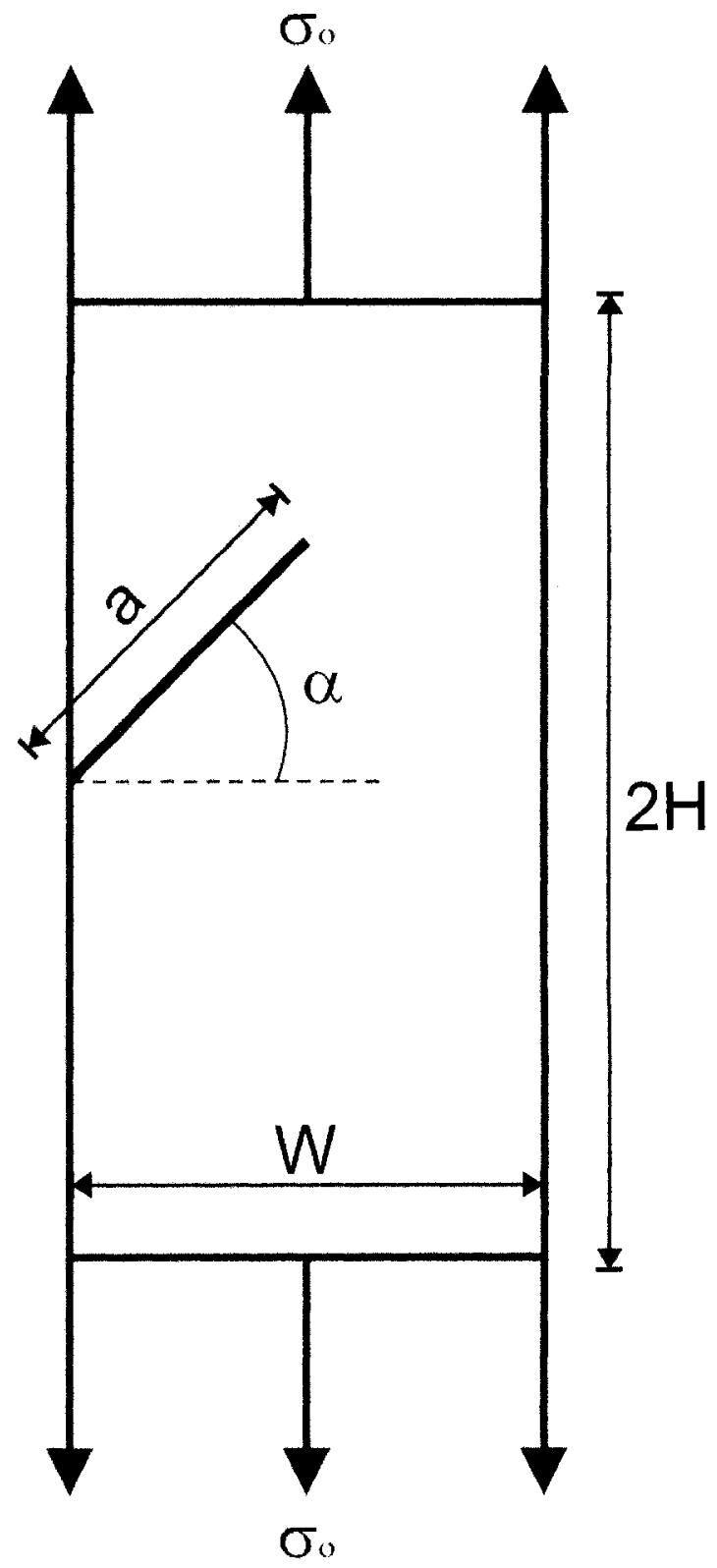

(b)

Figure 2.10: Geometries for the (a) CCP and (b) SECP specimen 


\section{Chapter 3}

\section{Stress Intensity Factor and T-Stress Solutions for Mixed-Mode Test Specimens Subjected to Remote Tension Loading}

In this chapter, the stress intensity factor (SIF) and T-stress values are determined at a variety of crack lengths and orientations for two different types of mixed-mode test specimens that are subjected to uniform tension using finite element analysis (FEA). The FEA modeling of the specimens is described, and the SIF and T-stress values obtained are validated against published works. The relationship between the T-stress values and both the crack length and crack orientation for each specimen is investigated and discussed.

\subsection{Specimen Geometries}

The mode I and mode II SIF's and T-stress values are determined for a center cracked plate (CCP) specimen (Fig. 3.1) and a single-edge cracked plate (SECP) specimen (Fig. 3.2) with a uniformly distributed tension, $\sigma_{o}$ applied at their ends. The CCP specimen used is a rectangular plate with an $H / W$ of 4 and a crack length of $2 a$, while the SECP specimen used is a rectangular plate with an $H / W$ of 2 with a crack of length $a$, where $H$ is half the specimen's total height and $W$ is half the total width for the CCP specimen and the total width for the SECP specimen.

The material properties used for the specimens are that of an isotropic homogeneous linear elastic material with a Young's modulus of $3 \times 10^{7} \mathrm{psi}$ (207 GPa) and a Poisson's ratio of 0.3 , both of which are typical values for steel. 
The SIF and T-stress values are obtained for both specimens for cracks with $a / W$ $=0.1,0.2,0.4,0.6$, and 0.8 and oriented at $\alpha=0^{\circ}, 15^{\circ}, 30^{\circ}, 45^{\circ}, 60^{\circ}$, and $75^{\circ}$, where $\alpha$ is the crack orientation angle with respect to the horizontal.

\subsection{Finite Element Analysis}

This section describes the process of FEA modeling on the geometries of the CCP and SECP specimens described above using ABAQUS/Standard (v. 6.6) to determine the mode I and mode II SIF's and T-stress values. Validation of the FEA against published results from Kitagawa \& Yuuki [1977], Bowie [1973] and Wang [2001] are also summarized.

\subsubsection{FEA Modeling}

The FEA is performed using ABAQUS/Standard (v. 6.6) [ABAQUS/CAE Users Manual, 2006]. ABAQUS/Standard CAE (v. 6.6) provides a Graphical User Interface (GUI) to better visualize and in many ways simplify the creation of parts, assemblies, associated boundary and loading conditions, and features such as cracks, as well as provide an efficient means of creating a viable mesh, all through an intuitive easy-to-use environment.

To model the CCP and SECP specimens described in section 3.1 above for an FEA analysis using ABAQUS/Standard CAE, a 2D planar deformable shell part of each specimen is first created one at a time. An isotropic linear elastic material with a Young's modulus of $3 \times 10^{7} \mathrm{psi}$ and a Poisson's ratio of 0.3 is then created and assigned to each part as a solid homogeneous section. Boundary and loading conditions are also created and assigned to the part as shown in Figure 3.3 for both the CCP and SECP specimen. The load used at the plate ends is a uniformly distributed load of $100 \mathrm{psi} / \mathrm{in}$.

A crack is modeled on the part and assigned "crack" properties, such as, the location of the crack-tip and crack-front (first contour region), as well as the crack extension direction. The contour integral is also assigned properties, which included the 
position of the mid-side node and the type of degeneracy of the element side at the cracktip. In this case, since the model is linear elastic with a $1 / \sqrt{r}$ singularity at the cracktip(s), the mid-side node of the elements in the first contour is located at the quarter-point and the sides of those elements located at the crack-tip are collapsed (degenerated) to a single node forming triangular elements (Section 2.4.1).

To model the region around the crack-tip more accurately, a refined sub-mesh is created around the crack-tip (Fig. 3.4-3.7). The refined sub-mesh provides more accurate results and faster convergence of those results over consecutive contour integrals.

The final step before the FEA analysis is to create the mesh. Meshing the part involves creating finite elements and assigning them to the model. The elements chosen to mesh the model are 2D solid (continuum) 8-node isoparametric elements. The elements are plane-strain, bi-quadratic, and quadrilateral. Typical FEA mesh for a CCP and SECP specimen for a crack oriented at $\alpha=0^{\circ}$ and $\alpha=15^{\circ}$ for $a / W=0.4$ are shown in Fig. 3.8 and 3.9.

In the FEA analysis phase, the SIF's and T-stress are extracted from the J-integral using an interaction integral (Section 2.4.2).

\subsubsection{Validation of FEA Model}

The FEA procedure is validated using FEA models with a relatively coarse mesh. The SIF and T-stress values calculated from the FEA models are compared with published values.

The SIF results from the FEA for the CCP and SECP specimens are validated against those published by Kitagawa \& Yuuki [1977], and Bowie [1973] respectively, while the T-stress FEA results for both CCP and SECP specimens are validated against those of Wang [2001]. 
For the purposes of validating the FEA procedure, the CCP and SECP specimens used for the purposes of the FEA and those used by the above-mentioned authors are the same. The specimens can be viewed in Figures 3.1 and 3.2. To compare the SIF's, the CCP specimen is modeled with an $H / W$ of 2 and the SECP specimen is modeled with an $H / W$ of 1 so that they correspond with the models used by Kitagawa \& Yuuki [1977], and Bowie [1973] respectively, while an $H / W$ of 4 is used to model both the CCP and SECP specimens for comparing T-stress values with Wang [2001] who used an $H / W$ of 3 . From the theory of long plates, comparing results from geometries with $H / W$ of 4 and 3 is acceptable.

The results are given in terms of normalized non-dimensional parameters given by the following relations:

Mode I SIF $\left(K_{\mathrm{I}}\right)$ normalized as:

$$
f_{1}=\frac{K_{I}}{\sigma_{o} \sqrt{\pi a}}
$$

Mode II SIF $\left(K_{\text {II }}\right)$ normalized as:

$$
f_{2}=\frac{K_{I I}}{\sigma_{o} \sqrt{\pi a}}
$$

T-stress $(T)$ normalized as:

$$
t=\frac{T}{\sigma_{o}}
$$

Where, $\sigma_{o}$ is the applied force per unit length along the ends of each specimen, and $a$ is the crack length. 


\section{CCP Specimen}

Comparison of the normalized mode I and mode II SIF's between the FEA and the results of Kitagawa \& Yuuki [1977] are shown in Tables 3.1 and 3.2 respectively. The normalized SIF's are compared for $a / W=0.2,0.4,0.6$, and 0.8 at $\alpha=0^{\circ}, 30^{\circ}$, and $60^{\circ}$. The maximum difference in either mode I or mode II SIF's is $1.49 \%$.

The normalized T-stress comparison between the FEA and those of Wang [2001] are performed for $a / W=0.2,0.4,0.6$, and 0.8 at $\alpha=0^{\circ}$. The comparison is shown on Table 3.3. The maximum difference in normalized T-stress is $0.46 \%$.

\section{SECP Specimens}

Comparisons similar to those carried out on the $\mathrm{CCP}$ specimens above are performed except for $a / W=0.8$ as data is not available. The FEA normalized mode I and mode II SIF's are compared to Bowie [1973]. Tables 3.4 and 3.5 show the comparisons. The maximum difference in either mode I or mode II SIF's is $2.28 \%$.

The FEA normalized T-stress is again compared to Wang [2001] with the results displayed in Table 3.6. The maximum difference in normalized T-stress is $2.39 \%$.

As the maximum difference between all values are within $3 \%$, excellent correlation is found between FEA results and published literature. The comparisons validate the FEA procedure used for determining the SIF's and T-stress for the CCP and SECP specimens.

\subsection{Results and Discussion}

In this section, the SIF and T-stress results of the FEA performed on the CCP and SECP specimens using a refined mesh are provided and discussed. Note that in Figures 3.10-3.17 the symbols represent the data obtained from the FEA for those points and the curves are lines of fit linking the data points for the purpose of displaying any trends that may be present. 


\subsubsection{CCP Specimen}

\section{Mode I and Mode II Stress Intensity Factor Results}

The mode I SIF $\left(K_{\mathrm{I}}\right)$ results normalized as $f_{I}(\mathrm{Eqn}$ 3.1) for the CCP specimen are shown in Tables 3.7, and Figure 3.10, while the mode II SIF $\left(K_{\text {II }}\right)$ results normalized as $f_{2}$ (Eqn 3.2) can be viewed in Table 3.8, and Figure 3.11.

From Figures 3.10a and 3.11a, $f_{I}$ and $f_{2}$ gradually increase with $a / W$ for a given $\alpha$, and as $\alpha$ increases the $f_{I}$ and $f_{2}$ curves seem to linearize; the $f_{I}$ curves comes close to being horizontal and the increase of $f_{l}$ with $a / W$ gets larger as $\alpha$ decreases (Fig. 3.10a). The $f_{2}$ curves (Fig. 3.11a) for $\alpha=30^{\circ}$ and $\alpha=60^{\circ}$, as well as the curves for $\alpha=15^{\circ}$ and $\alpha=75^{\circ}$ intersect each other at $a / W=0.1$ and $a / W \approx 0.75$.

Referring to Figure $3.10 \mathrm{~b}, f_{l}$ steadily decreases with an increase in $\alpha$ and $f_{l}$ seems to converge to a value of 0 as $\alpha$ approaches $90^{\circ}$ for all $a / W$, while Figure $3.11 \mathrm{~b}$ shows that $f_{2}$ increases from $\alpha=15^{\circ}$ to $\alpha=45^{\circ}$ and then decreases from $\alpha=45^{\circ}$ to $\alpha=75^{\circ}$. The $f_{2}$ value tends to want to converge to 0 as $\alpha$ approach $90^{\circ}$. At $\alpha=0^{\circ} f_{2}$ is 0 (pure mode I).

\section{T-Stress Results}

The T-stress results normalized as $t$ (Eqn 3.3) for the CCP specimen can be seen in Table 3.9, and Figures 3.12 and 3.13.

From Figure 3.12, $t \leq 0$ for $\alpha \leq 45^{\circ}$ and $t>0$ for $\alpha>45^{\circ}$. In general, $t$ decreases as $a / W$ increases, and as $\alpha$ increases, the curves linearize somewhat and become horizontal. The largest change in $t$ is from $a / W=0.6$ to $a / W=0.8$ for $\alpha=0^{\circ}$ and $\alpha=15^{\circ}$, while for all other angles and $a / W$ the change in $t$ is gradual and steady.

Figure 3.13, shows that $t$ increases with $\alpha$ and tends to want to converge to 1 as $\alpha$ approaches $90^{\circ}$. The normalized T-stress $t$ for a given $a / W$ becomes more positive as $\alpha$ increases. Both Figure 3.12 and 3.13 show that the transition of $t$ from negative to positive is dependent predominantly on $\alpha$. 


\section{Discussion}

The trends displayed by mode I and mode II SIF's for the CCP specimen are the same as those obtained by Kitagawa \& Yuuki [1977]. The convergence of the mode I and mode II SIF's to 0, and the convergence on the normalized T-stress to 1 (a pure T-stress state), as the crack angle goes to $90^{\circ}$ is the result of the crack lining up with the direction of the stress field created by the applied load. The convergence of the normalized T-stress to 1 represents the magnitude and direction of the stress field exerted by the applied loading.

The T-stress results are observed to be significant for almost every loading condition investigated. Due to the observed significance of the T-stress value, they should be taken into account to ensure better accuracy in fracture analysis.

\subsubsection{SECP Specimen}

\section{Mode I and Mode II Stress Intensity Factor Results}

The mode I SIF $\left(K_{\mathrm{I}}\right)$ results normalized as $f_{l}(\operatorname{Eq} \underline{n} 3.1)$ for the SECP specimen are shown in Tables 3.10, and Figure 3.14, while the mode II SIF ( $\left.K_{\mathrm{II}}\right)$ results normalized as $f_{2}$ (Eqn 3.2) can be viewed in Table 3.11, and Figure 3.15.

Figures 3.14a and 3.15a show that $f_{l}$ and $f_{2}$ increase with $a / W$ for a given $\alpha$; the increase is gradual for all $\alpha$ except for $\alpha=0^{\circ}$ and $\alpha=15^{\circ}$ where it increases rapidly between $a / W=0.6$ to $a / W=0.8$. The $f_{I}$ curves become more linear as $\alpha$ increases until it becomes horizontal at $\alpha=75^{\circ}$ (Fig. 3.14a). For the $f_{2}$ curves (Fig. 3.15a), The $\alpha=15^{\circ}$ curve intersects the $\alpha=45^{\circ}, \alpha=60^{\circ}$, and $\alpha=75^{\circ}$ curves, while the $\alpha=30^{\circ}$ curve intersects the $\alpha=45^{\circ}$ and $\alpha=60^{\circ}$ curves; the curves tend become more linear as $\alpha$ increases.

Referring to Figure 3.14b, $f_{l}$ decreases with increase in $\alpha$ and converges to 0 as $\alpha$ approaches $90^{\circ}$; the decrease is gradual for all but $a / W=0.8$, while, from Figure 3.15b, $f_{2}$ increases with $\alpha$ as it goes from $15^{\circ}$ to about $30^{\circ}$ and then converges to 0 as $\alpha$ 
approaches $90^{\circ} ; a / W=0.8$ converges more sharply than all other $a / W$ curves. The value of $f_{2}$ is 0 at $\alpha=0^{\circ}$ (pure mode I). Note that, for an SECP specimen, a crack oriented at $90^{\circ}$ corresponds to the specimen's edge and is not a crack at all.

\section{T-Stress Results}

The T-stress results normalized as $t$ (Eqn 3.3) for the SECP specimen can be seen in Table 3.12, and Figures 3.16 and 3.17.

Looking at Figure 3.16, $t$ increases with $a / W$ in general; the increase in $t$ is much more pronounced for $\alpha=0^{\circ}, \alpha=15^{\circ}$, and $\alpha=30^{\circ}$ from $a / W=0.6$ to $a / W=0.8$. As $\alpha$ increases the curves become more linear and horizontal. The value of $t$ for the $\alpha=45^{\circ}$, $\alpha=60^{\circ}$, and $\alpha=75^{\circ}$ curves are all positive, while for the $\alpha=0^{\circ}$ and $\alpha=30^{\circ}$ curves $t$ is positive above $a / W \approx 0.6$, and for the $\alpha=15^{\circ}$ curve $t$ is positive above $a / W=0.4$. Note that all the curves approximately intersect each other at $a / W \approx 0.65$ and $t \approx 1$.

From Figure 3.17, $t$ gradually increases with $\alpha$ for all but the $a / W=0.8$ curve, which decreases rapidly with $\alpha$. All curves converge to $t=1$ as $\alpha$ approaches $90^{\circ}$ (nocrack orientation). The value of $t$ for the $a / W=0.6$ and $a / W=0.8$ curves are all positive while for the $a / W=0.1, a / W=0.2$, and $a / W=0.4$ curves, $t$ is positive only above $\alpha \approx 30^{\circ}$.

For the SECP specimen, the transition from negative to positive T-stress is equally dependent on both the crack orientation and the relative crack length.

\section{Discussion}

The trends displayed by mode I and mode II SIF's for the CCP specimen are the same as those obtained by Bowie [1973]. A crack oriented at $90^{\circ}$ to the horizontal in a SECP specimen is a crack that is congruent with the specimen's edge and therefore physically represents a specimen with no crack. In such a "no-crack" situation, as expected, the mode I and mode II SIF's would converge to 0 , and the normalized T-stress 
would converge to 1 . Physically, as there is no crack at an orientation of $90^{\circ}$, by definition there is no T-stress. Thus, the convergence of the normalized T-stress to 1 as the crack orientation approaches $90^{\circ}$ represents the stress exerted by the applied force on the specimen.

As with the CCP specimen, The T-stress results are observed to be significant for almost every loading scenario tested and therefore need to be considered to ensure a comprehensive fracture analysis.

The significant difference in trend shown by the $a / W=0.8$ compared to all other curves is due to the external boundary, i.e., the edge opposite the crack, exerting an influence on the conditions at the crack-tip.

\subsection{Conclusions}

Mode I and mode II stress intensity factors and T-stress values for the CCP and SECP specimens are determined for a variety of crack lengths $(a / W=0.1,0.2,0.4,0.6$, and 0.8 ) at different orientations $\left(\alpha=0^{\circ}, 15^{\circ}, 30^{\circ}, 45^{\circ}, 60^{\circ}\right.$, and $75^{\circ}$ with respect to the horizontal) using finite element analysis. The values are validated against results published by Kitagawa \& Yuuki [1977], Bowie [1973], and Wang [2001]. The FEA results showed good correlation published results.

The T-stress transition from negative to positive is predominantly dependent on the orientation of the crack for the CCP specimen, while for the SECP specimen the transition is dependent on both the relative crack size and the crack's orientation. The Tstress values are found to be significant in many of the testing scenarios such that they need to be taken into account if better accuracy is required in fatigue and fracture analysis. 
Table 3.1: Comparison of $f_{1}$ values from coarse mesh FEA and Kitagawa \& Yuuki [1977] for the CCP specimen

\begin{tabular}{|c|c|c|c|c|c|c|c|c|c|}
\hline \multirow{2}{*}{$a / W$} & \multicolumn{3}{|c|}{$\alpha=0^{\circ}$} & \multicolumn{3}{|c|}{$\alpha=30^{\circ}$} & \multicolumn{3}{|c|}{$\alpha=60^{\circ}$} \\
\hline & $\begin{array}{c}\text { Solution } \\
\text { from } \\
\text { Literature } \\
, f_{i}^{*} \\
\end{array}$ & $\begin{array}{c}\begin{array}{c}\text { Solution } \\
\text { from }\end{array} \\
\text { FEA, } \\
f_{l} \\
\end{array}$ & $\begin{array}{c}\text { Absolute } \\
\text { Difference } \\
(\%)\end{array}$ & $\begin{array}{c}\text { Solution } \\
\text { from } \\
\text { Literature, } \\
f_{l}^{*} \\
\end{array}$ & $\begin{array}{c}\text { Solution } \\
\text { from } \\
\text { FEA, } \\
f_{1} \\
\end{array}$ & $\begin{array}{c}\text { Absolute } \\
\text { Difference } \\
(\%)\end{array}$ & $\begin{array}{c}\text { Solution } \\
\text { from } \\
\text { Literature, } \\
f_{1}^{*} \\
\end{array}$ & $\begin{array}{c}\begin{array}{c}\text { Solution } \\
\text { from }\end{array} \\
\text { FEA, } \\
f_{l} \\
\end{array}$ & $\begin{array}{c}\text { Absolute } \\
\text { Difference } \\
(\%)\end{array}$ \\
\hline 0.2 & 1.02 & 1.0251 & 0.50 & 0.7730 & 0.7733 & 0.04 & 0.2605 & 0.2605 & 0.00 \\
\hline 0.4 & 1.11 & 1.1106 & 0.05 & 0.8456 & 0.8459 & 0.04 & 0.2896 & 0.2897 & 0.03 \\
\hline 0.6 & 1.30 & 1.3045 & 0.35 & 0.984 & 0.9840 & 0.00 & 0.3332 & 0.3332 & 0.00 \\
\hline 0.8 & 1.79 & 1.8167 & 1.49 & 1.245 & 1.2439 & 0.09 & 0.388 & 0.3881 & 0.03 \\
\hline
\end{tabular}


Table 3.2: Comparison of $f_{2}$ values from coarse mesh FEA and Kitagawa \& Yuuki [1977] for the CCP specimen

\begin{tabular}{|c|c|c|c|c|c|c|}
\hline \multirow{2}{*}{$a / W$} & \multicolumn{3}{|c|}{$\alpha=30^{\circ}$} & \multicolumn{3}{|c|}{$\alpha=60^{\circ}$} \\
\hline & $\begin{array}{c}\text { Solution } \\
\text { from } \\
\text { Literature, } \\
f_{2}^{*} \\
\end{array}$ & $\begin{array}{c}\text { Solution } \\
\text { from } \\
\text { FEA, } \\
f_{2}\end{array}$ & $\begin{array}{c}\text { Absolute } \\
\text { Difference } \\
(\%)\end{array}$ & $\begin{array}{c}\text { Solution } \\
\text { from } \\
\text { Literature, } \\
f_{2}^{*} \\
\end{array}$ & $\begin{array}{c}\text { Solution } \\
\text { from } \\
\text { FEA, } \\
f_{2}\end{array}$ & $\begin{array}{c}\text { Absolute } \\
\text { Difference } \\
(\%)\end{array}$ \\
\hline 0.2 & 0.4367 & 0.4369 & 0.05 & 0.4417 & 0.4419 & 0.05 \\
\hline 0.4 & 0.4497 & 0.4500 & 0.07 & 0.4660 & 0.4662 & 0.04 \\
\hline 0.6 & 0.480 & 0.4803 & 0.06 & 0.5022 & 0.5024 & 0.04 \\
\hline 0.8 & 0.550 & 0.5526 & 0.47 & 0.549 & 0.5492 & 0.04 \\
\hline
\end{tabular}


Table 3.3: Comparison of $t$ values from coarse mesh FEA and Wang [2002] for the CCP specimen

\begin{tabular}{lccc}
\hline$a / W$ & & $\alpha=0^{\circ}$ & \\
\cline { 2 - 4 } & $\begin{array}{c}\text { Solution from } \\
\text { Literature, } t^{*}\end{array}$ & $\begin{array}{c}\text { Solution from FEA, } \\
t\end{array}$ & $\begin{array}{c}\text { Absolute Difference } \\
(\%)\end{array}$ \\
\hline 0.2 & -1.0289 & -1.031 & 0.20 \\
0.4 & -1.1394 & -1.144 & 0.40 \\
0.6 & -1.4484 & & 0.46 \\
0.8 & & -1.455 & \\
& -2.5689 & & 0.07 \\
\hline
\end{tabular}


Table 3.4: Comparison of $f_{1}$ values from coarse mesh FEA and Bowie [1973] for the SECP specimen

\begin{tabular}{|c|c|c|c|c|c|c|c|c|c|}
\hline \multirow{2}{*}{$a / W$} & \multicolumn{3}{|c|}{$\alpha=0^{\circ}$} & \multicolumn{3}{|c|}{$\alpha=30^{\circ}$} & \multicolumn{3}{|c|}{$\alpha=60^{\circ}$} \\
\hline & $\begin{array}{c}\text { Solution } \\
\text { from } \\
\text { Literature, } \\
f_{1}^{*} \\
\end{array}$ & $\begin{array}{c}\begin{array}{c}\text { Solution } \\
\text { from }\end{array} \\
\text { FEA, } \\
f_{l}\end{array}$ & $\begin{array}{c}\text { Absolute } \\
\text { Difference } \\
(\%)\end{array}$ & $\begin{array}{c}\text { Solution } \\
\text { from } \\
\text { Literature, } \\
f_{1}^{*} \\
\end{array}$ & $\begin{array}{c}\text { Solution } \\
\text { from } \\
\text { FEA, } \\
f_{1} \\
\end{array}$ & $\begin{array}{c}\text { Absolute } \\
\text { Difference } \\
(\%)\end{array}$ & $\begin{array}{c}\text { Solution } \\
\text { from } \\
\text { Literature, } \\
f_{l}^{*} \\
\end{array}$ & $\begin{array}{c}\begin{array}{c}\text { Solution } \\
\text { from }\end{array} \\
\text { FEA, } \\
f_{l} \\
\end{array}$ & $\begin{array}{c}\text { Absolute } \\
\text { Difference } \\
(\%)\end{array}$ \\
\hline 0.2 & 1.02 & 1.0251 & 0.50 & 0.7730 & 0.7733 & 0.04 & 0.2605 & 0.2605 & 0.00 \\
\hline 0.4 & 1.11 & 1.1106 & 0.05 & 0.8456 & 0.8459 & 0.04 & 0.2896 & 0.2897 & 0.03 \\
\hline 0.6 & 1.30 & 1.3045 & 0.35 & 0.984 & 0.9840 & 0.00 & 0.3332 & 0.3332 & 0.00 \\
\hline
\end{tabular}


Table 3.5: Comparison of $f_{2}$ values from coarse mesh FEA and Bowie [1973] for the SECP specimen

\begin{tabular}{|c|c|c|c|c|c|c|}
\hline \multirow{2}{*}{$a / W$} & \multicolumn{3}{|c|}{$\alpha=30^{\circ}$} & \multicolumn{3}{|c|}{$\alpha=60^{\circ}$} \\
\hline & $\begin{array}{c}\text { Solution } \\
\text { from } \\
\text { Literature, } \\
f_{2}^{*} \\
\end{array}$ & $\begin{array}{c}\begin{array}{c}\text { Solution } \\
\text { from }\end{array} \\
\text { FEA, } \\
f_{2} \\
\end{array}$ & $\begin{array}{c}\text { Absolute } \\
\text { Difference } \\
(\%)\end{array}$ & $\begin{array}{c}\text { Solution } \\
\text { from } \\
\text { Literature, } \\
f_{2}^{*} \\
\end{array}$ & $\begin{array}{c}\text { Solution } \\
\text { from } \\
\text { FEA, } \\
f_{2} \\
\end{array}$ & $\begin{array}{c}\text { Absolute } \\
\text { Difference } \\
(\%)\end{array}$ \\
\hline 0.2 & 0.353 & 0.3535 & 0.14 & 0.352 & 0.3569 & 1.39 \\
\hline 0.4 & 0.473 & 0.4713 & 0.36 & 0.412 & 0.4108 & 0.29 \\
\hline 0.6 & 0.704 & 0.6913 & 1.80 & 0.498 & 0.4898 & 1.65 \\
\hline
\end{tabular}


Table 3.6: Comparison of $t$ values from coarse mesh FEA and Wang [2002] for the SECP specimen

\begin{tabular}{|c|c|c|c|}
\hline \multirow{2}{*}{$a / W$} & \multicolumn{3}{|c|}{$\alpha=0^{\circ}$} \\
\hline & $\begin{array}{l}\text { Solution from } \\
\text { Literature, } t^{*}\end{array}$ & $\begin{array}{c}\text { Solution from FEA, } \\
t\end{array}$ & $\begin{array}{l}\text { Absolute Difference } \\
(\%)\end{array}$ \\
\hline 0.2 & -0.5949 & -0.5892 & 0.01 \\
\hline 0.4 & -0.5782 & -0.5786 & 0.00 \\
\hline 0.6 & 0.0268 & -0.0373 & 2.39 \\
\hline 0.8 & 5.8298 & 6.006 & 0.03 \\
\hline
\end{tabular}


Table 3.7: $f_{l}$ values for CCP specimen from comprehensive FEA

\begin{tabular}{ccccccc}
\hline$a / W$ & $\alpha=0^{\circ}$ & $\alpha=15^{\circ}$ & $\alpha=30^{\circ}$ & $\alpha=45^{\circ}$ & $\alpha=60^{\circ}$ & $\alpha=75^{\circ}$ \\
\hline 0.1 & 1.0062 & 0.9393 & 0.7559 & 0.5046 & 0.2528 & 0.0678 \\
0.2 & 1.0249 & 0.9577 & 0.7731 & 0.5184 & 0.2606 & 0.0701 \\
0.4 & 1.1097 & 1.0401 & 0.8454 & 0.5721 & 0.2901 & 0.0785 \\
0.6 & 1.3038 & 1.2178 & 0.9833 & 0.6612 & 0.3342 & 0.0903 \\
0.8 & 1.8167 & 1.6407 & 1.2420 & 0.7942 & 0.3901 & 0.1041 \\
\hline
\end{tabular}

Table 3.8: $f_{2}$ values for CCP specimen from comprehensive FEA

\begin{tabular}{lllllll}
\hline$a / W$ & $\alpha=0^{\circ}$ & $\alpha=15^{\circ}$ & $\alpha=30^{\circ}$ & $\alpha=45^{\circ}$ & $\alpha=60^{\circ}$ & $\alpha=75^{\circ}$ \\
\hline 0.1 & & 0.2503 & 0.4339 & 0.5017 & 0.4353 & 0.2516 \\
0.2 & & 0.2509 & 0.4365 & 0.5072 & 0.4417 & 0.2561 \\
0.4 & 0 & 0.2555 & 0.4490 & 0.5282 & 0.4655 & 0.2721 \\
0.6 & & 0.2723 & 0.4788 & 0.5651 & 0.5004 & 0.2936 \\
0.8 & 0.3322 & 0.5510 & 0.6249 & 0.5437 & 0.3172 \\
\hline
\end{tabular}

Table 3.9: $t$ values for CCP specimen from comprehensive FEA

\begin{tabular}{ccccccc}
\hline$a / W$ & $\alpha=0^{\circ}$ & $\alpha=15^{\circ}$ & $\alpha=30^{\circ}$ & $\alpha=45^{\circ}$ & $\alpha=60^{\circ}$ & $\alpha=75^{\circ}$ \\
\hline 0.1 & -1.008 & -0.8744 & -0.5082 & 0.00666 & 0.4956 & 0.8648 \\
0.2 & -1.031 & -0.8975 & -0.5311 & -0.02596 & 0.4843 & 0.8615 \\
0.4 & -1.144 & -1.005 & -0.6206 & -0.08839 & 0.452 & 0.8529 \\
0.6 & -1.455 & -1.264 & -0.7736 & -0.1536 & 0.432 & 0.8487 \\
0.8 & -2.567 & -2.043 & -1.049 & -0.1864 & 0.4515 & 0.8594 \\
\hline
\end{tabular}


Table 3.10: $f_{l}$ values for SECP specimen from comprehensive FEA

\begin{tabular}{ccccccc}
\hline $\mathrm{a} / \mathrm{W}$ & $\alpha=0^{\circ}$ & $\alpha=15^{\circ}$ & $\alpha=30^{\circ}$ & $\alpha=45^{\circ}$ & $\alpha=60^{\circ}$ & $\alpha=75^{\circ}$ \\
\hline 0.1 & 1.1895 & 1.1290 & 0.9625 & 0.7274 & 0.4703 & 0.2328 \\
0.2 & 1.3675 & 1.2881 & 1.0751 & 0.7876 & 0.4923 & 0.2369 \\
0.4 & 2.1115 & 1.9447 & 1.5228 & 1.0178 & 0.5733 & 0.2511 \\
0.6 & 4.0330 & 3.5668 & 2.5114 & 1.4582 & 0.7101 & 0.2736 \\
0.8 & 11.9533 & 9.4239 & 5.1756 & 2.3396 & 0.9260 & 0.3032 \\
\hline
\end{tabular}

Table 3.11: $f_{2}$ values for SECP specimen from comprehensive FEA

\begin{tabular}{lllllll}
\hline$a / W$ & $\alpha=0^{\circ}$ & $\alpha=15^{\circ}$ & $\alpha=30^{\circ}$ & $\alpha=45^{\circ}$ & $\alpha=60^{\circ}$ & $\alpha=75^{\circ}$ \\
\hline 0.1 & & 0.1832 & 0.3194 & 0.3757 & 0.3418 & 0.2273 \\
0.2 & & 0.2068 & 0.3535 & 0.4043 & 0.3565 & 0.2310 \\
0.4 & 0 & 0.2939 & 0.4771 & 0.5054 & 0.4084 & 0.2438 \\
0.6 & & 0.4839 & 0.7214 & 0.6834 & 0.4909 & 0.2631 \\
0.8 & 1.1127 & 1.3259 & 1.0149 & 0.6150 & 0.2885 \\
\hline
\end{tabular}

Table 3.12: $t$ values for SECP specimen from comprehensive FEA

\begin{tabular}{ccccccc}
\hline$\alpha / W$ & $\alpha=0^{\circ}$ & $\alpha=15^{\circ}$ & $\alpha=30^{\circ}$ & $\alpha=45^{\circ}$ & $\alpha=60^{\circ}$ & $\alpha=75^{\circ}$ \\
\hline 0.1 & -0.5514 & -0.4276 & -0.09974 & 0.3259 & 0.7116 & 0.925 \\
0.2 & -0.5892 & -0.4519 & -0.09234 & 0.3575 & 0.7435 & 0.9383 \\
0.4 & -0.5786 & -0.4086 & 0.01779 & 0.505 & 0.8642 & 0.9907 \\
0.6 & -0.03733 & 0.1773 & 0.5169 & 0.8752 & 1.084 & 1.074 \\
0.8 & 6.006 & 4.748 & 2.864 & 1.873 & 1.473 & 1.187 \\
\hline
\end{tabular}




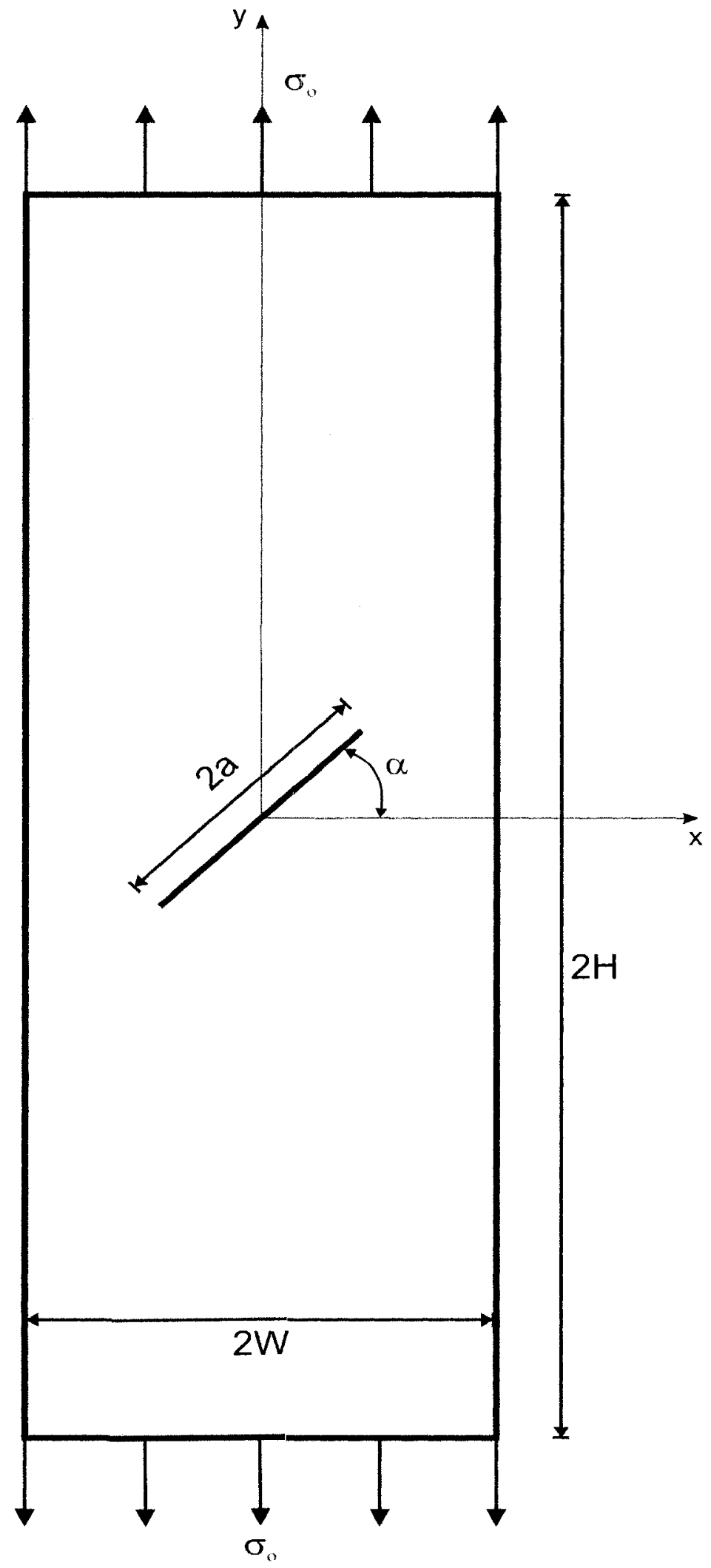

Figure 3.1: Center cracked plate (CCP) specimen 


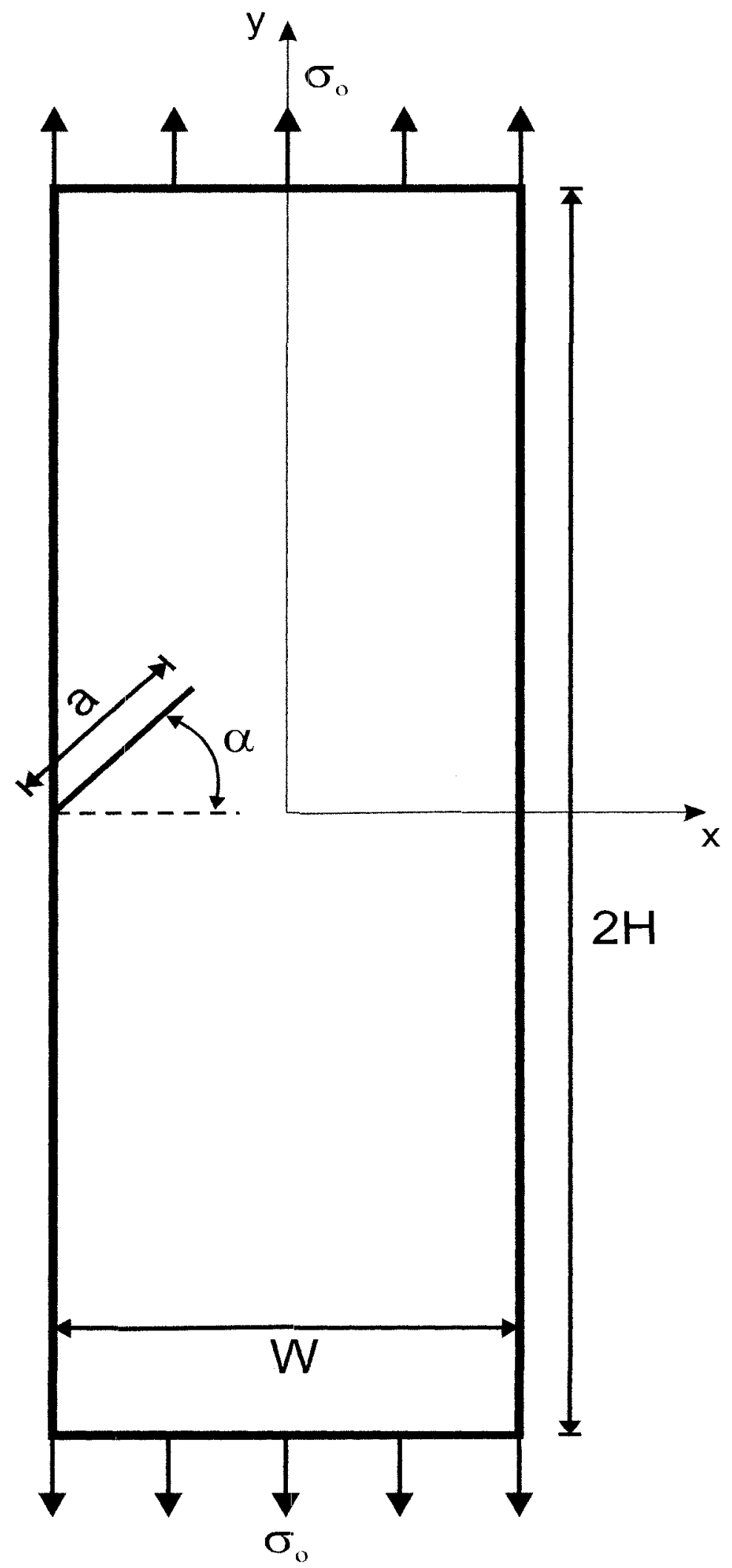

Figure 3.2: Single-edge cracked plate (SECP) specimen 


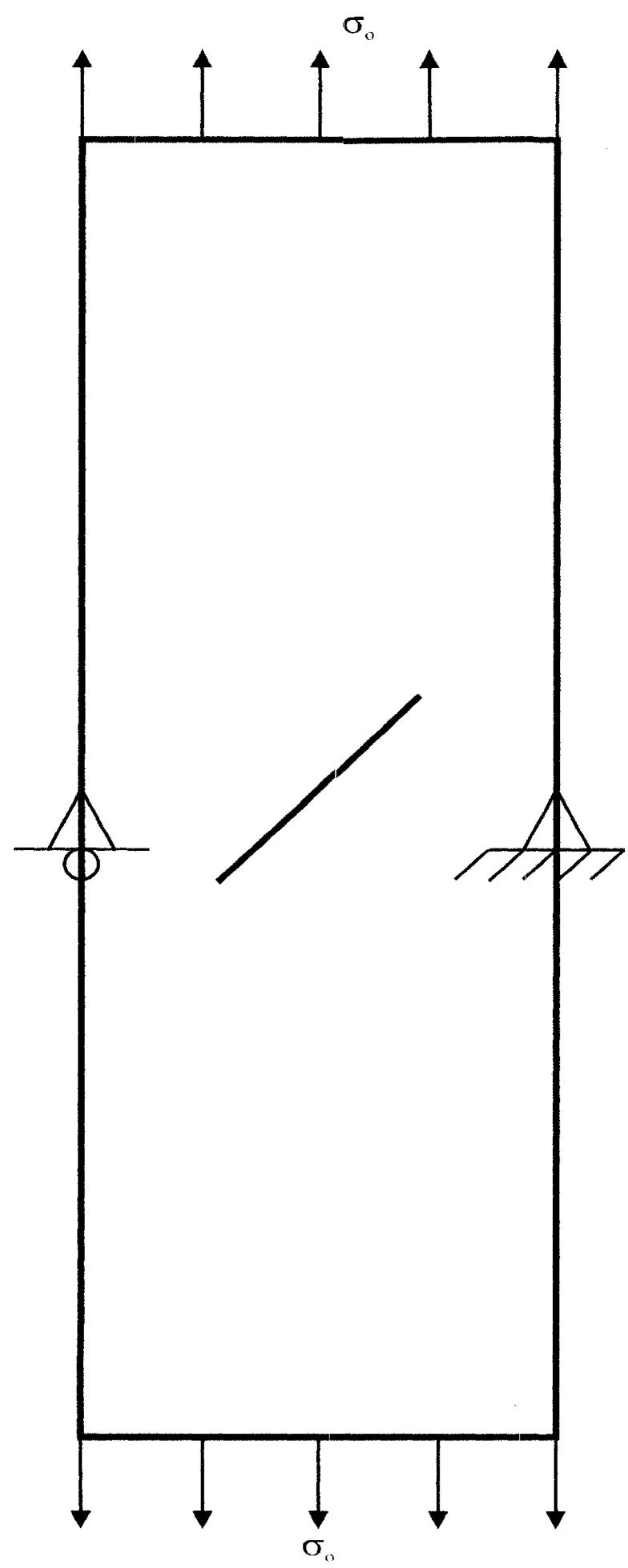

(a)

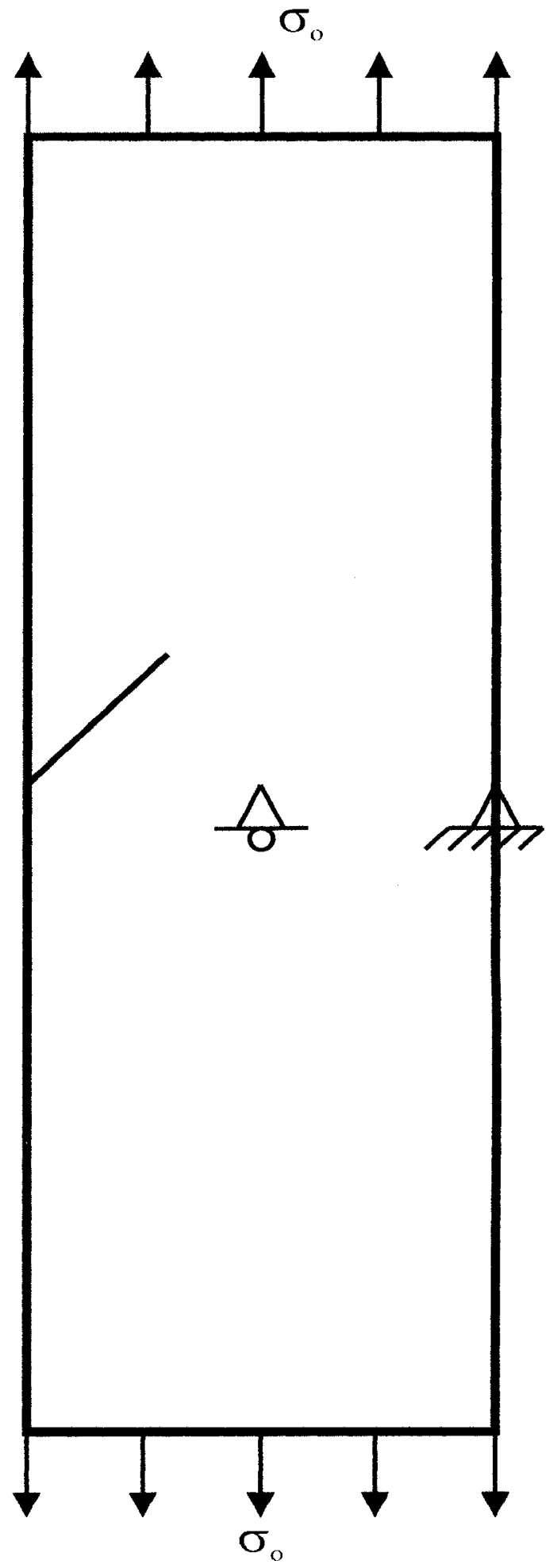

(b)

Figure 3.3: Boundary and loading conditions applied to (a) CCP and (b) SECP specimens for FEA 


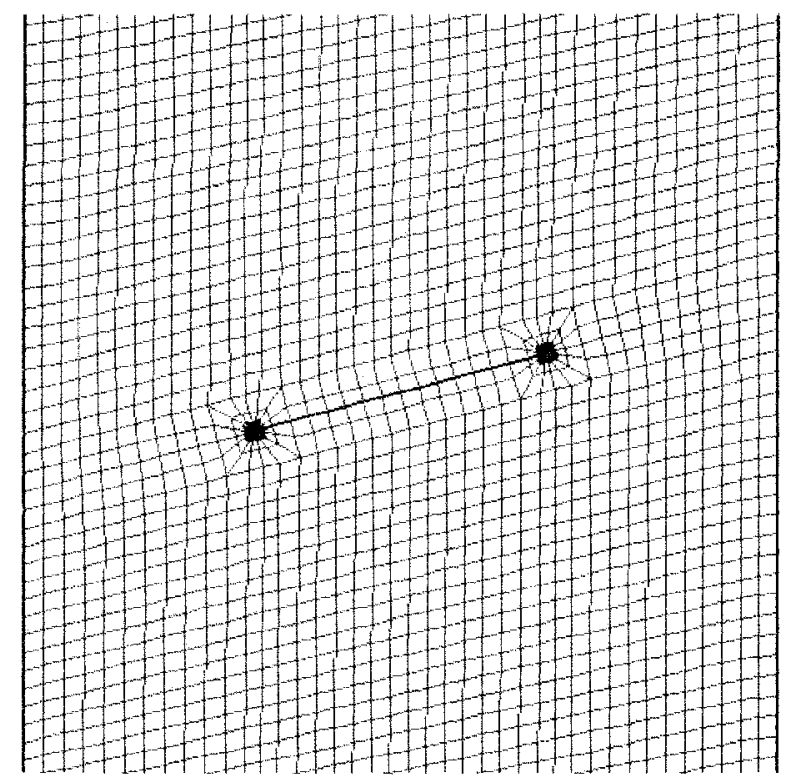

Figure 3.4: CCP FEA mesh magnified $4 x$ to show sub-mesh

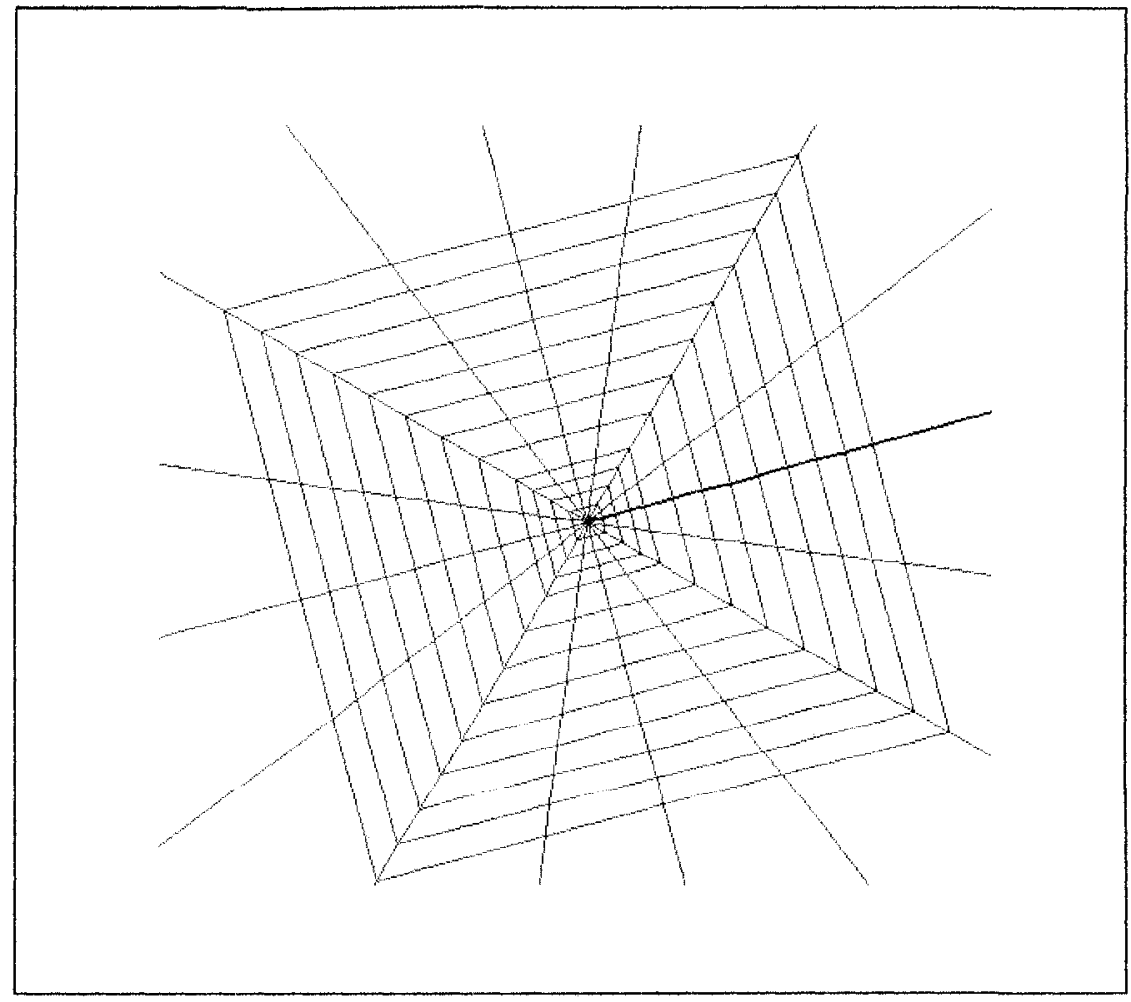

Figure 3.5: CCP FEA refined sub-mesh (Magnification 125x) 


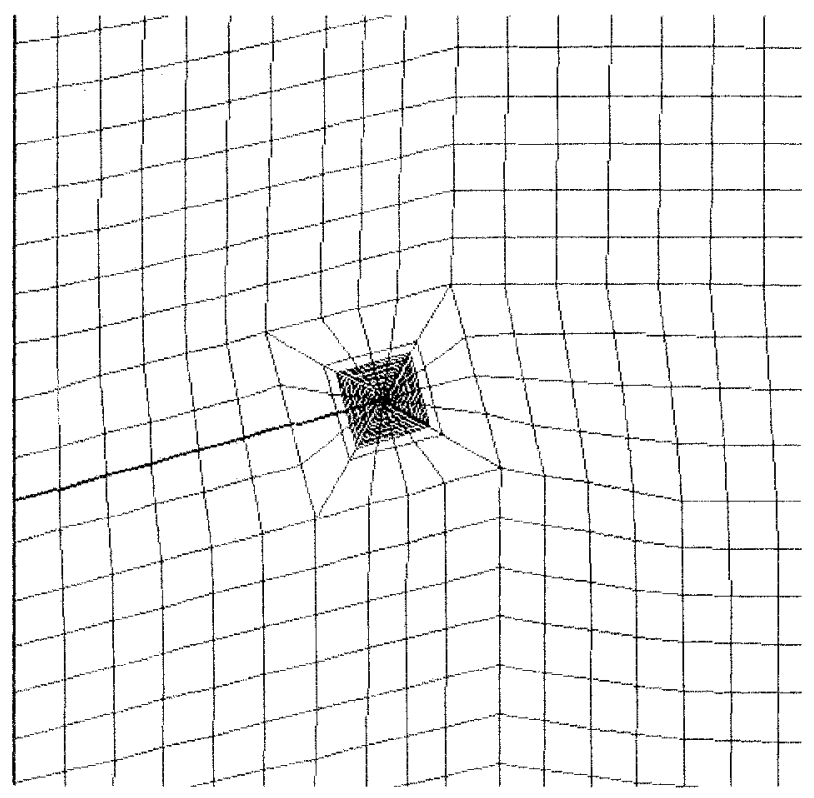

Figure 3.6: SECP FEA mesh magnified $5 x$ to show sub-mesh

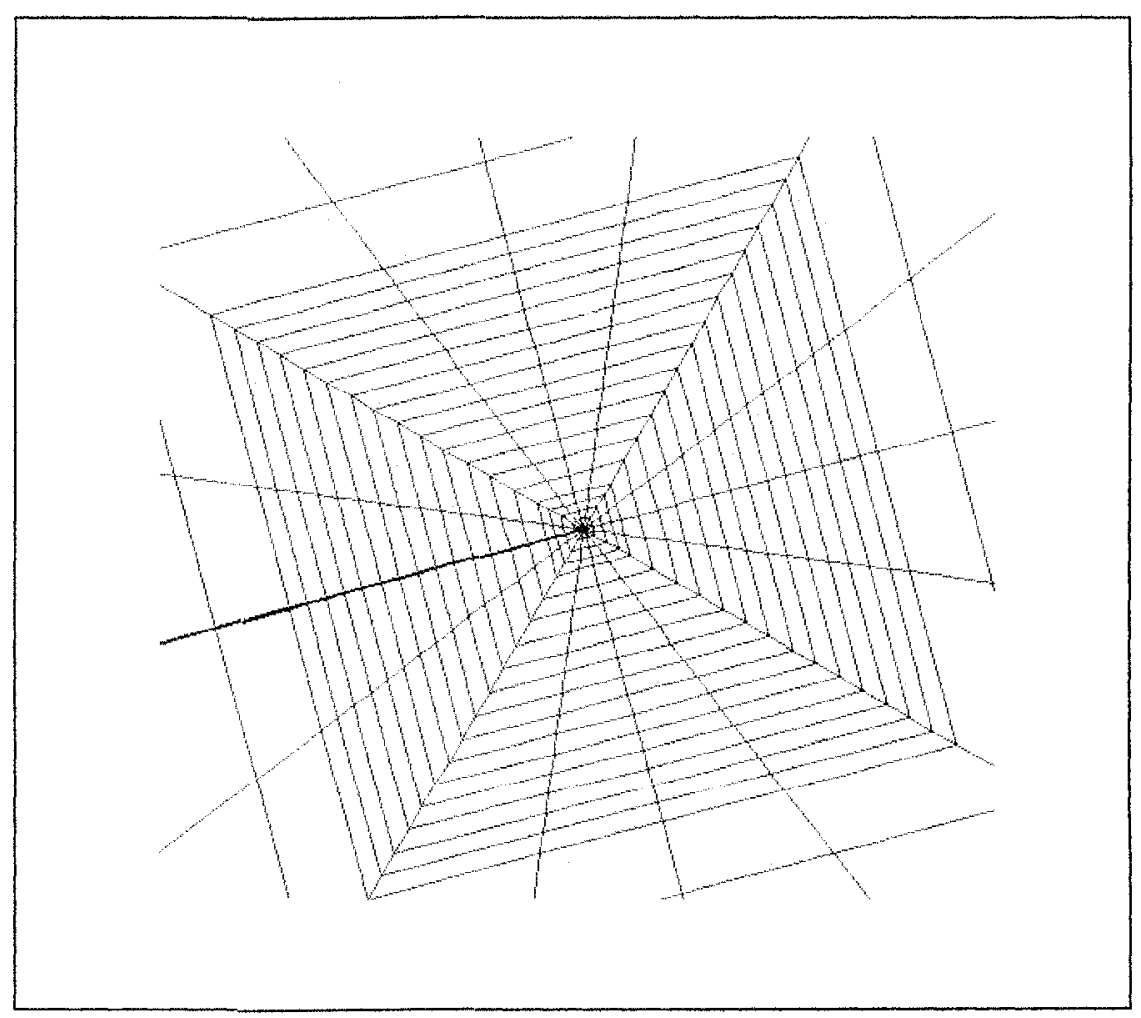

Figure 3.7: SECP FEA refined sub-mesh (Magnification 40x) 


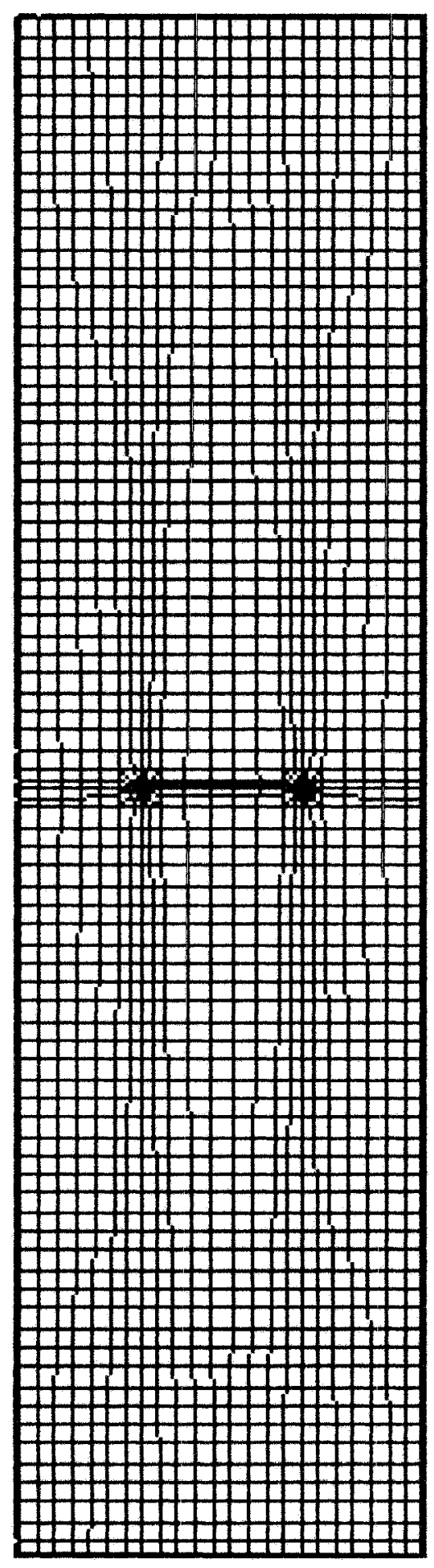

(a)

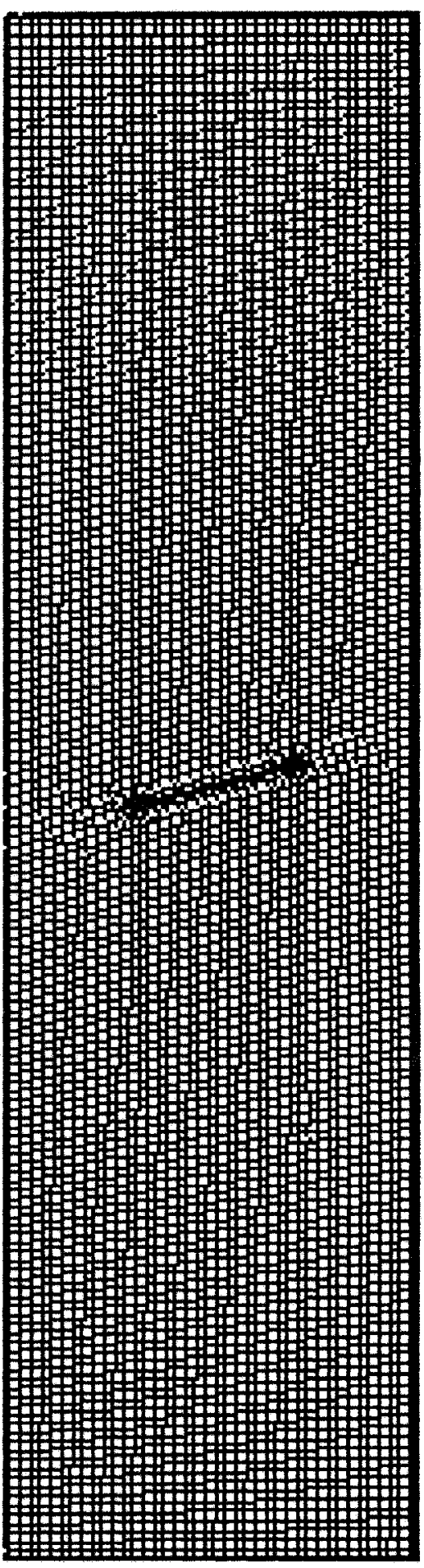

(b)

Figure 3.8: Typical mesh of a CCP specimen with $a / W=0.4$ oriented at (a) $\alpha=0^{\circ}$ and (b) $\alpha=15^{\circ}$ 


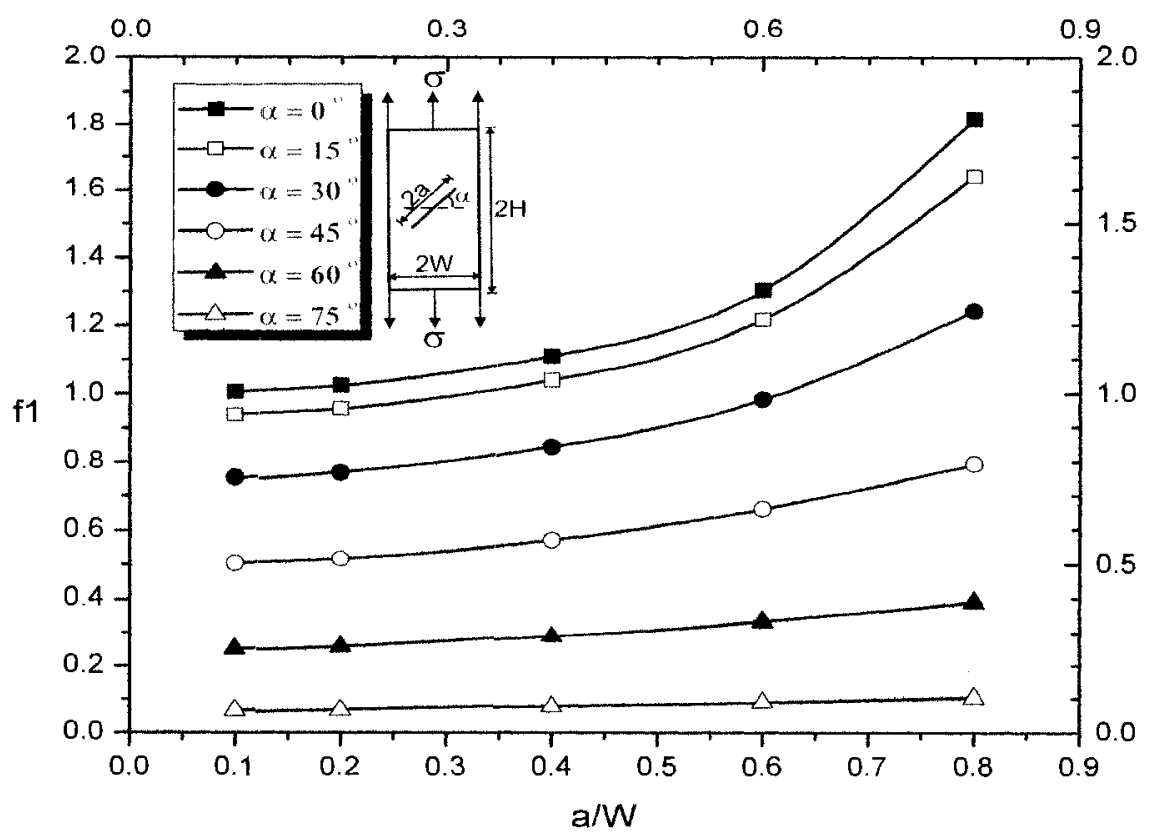

(a)

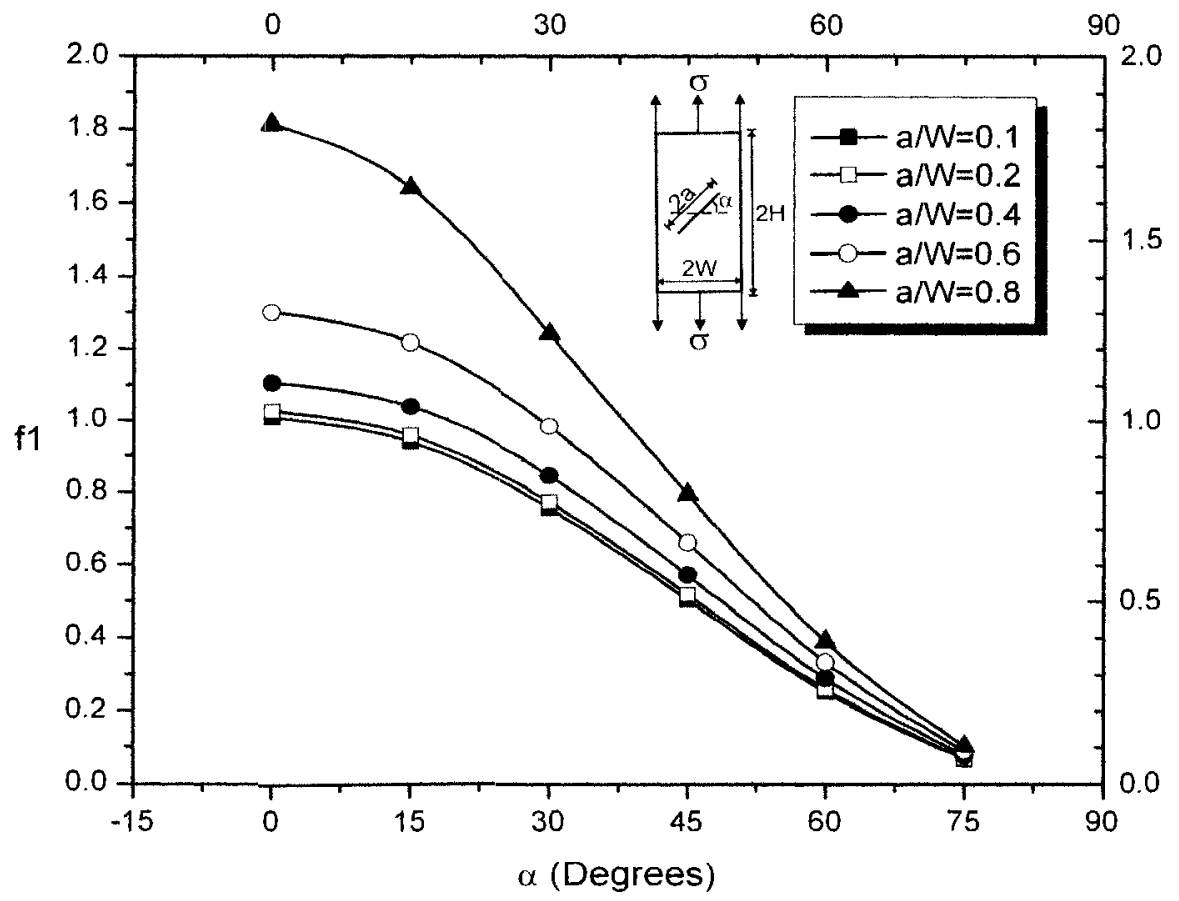

(b)

Figure 3.10: Normalized mode I SIF as a function of (a) $a / W$ and (b) $\alpha$ for the CCP specimen 


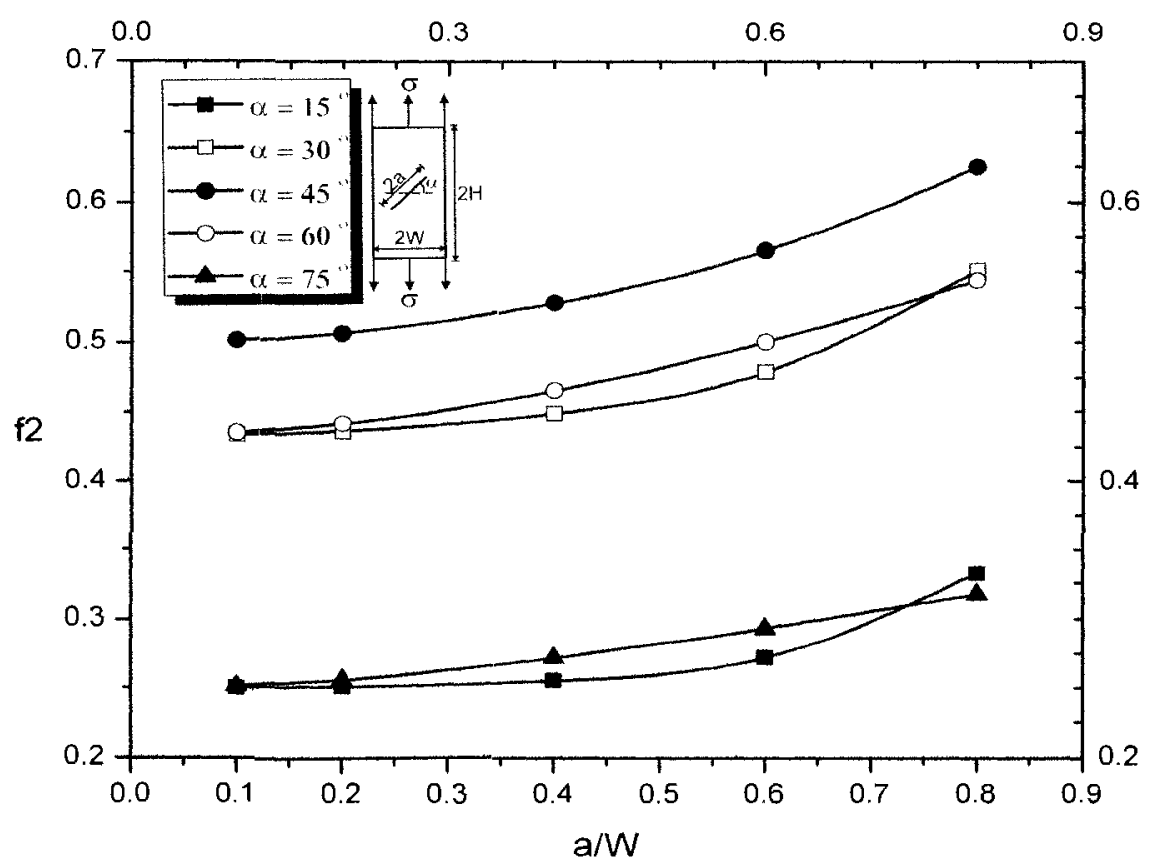

(a)

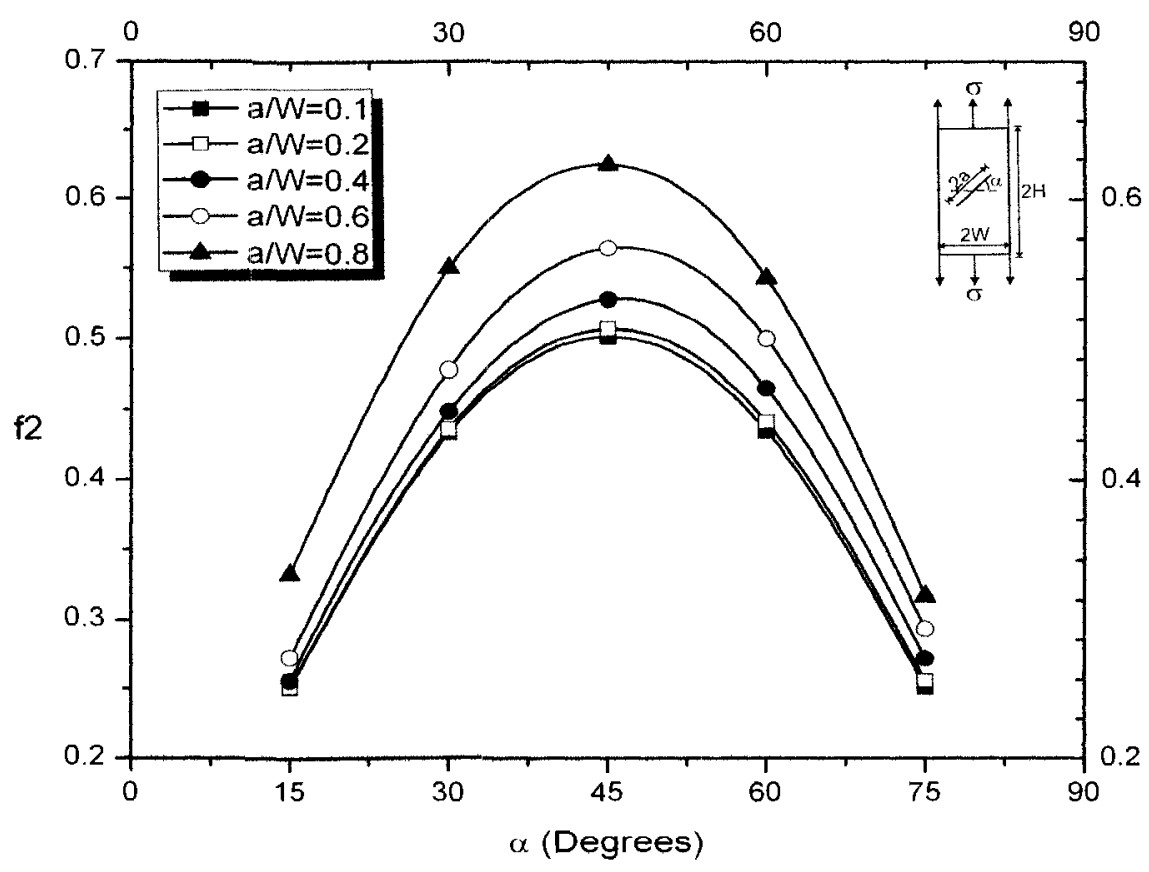

(b)

Figure 3.11: Normalized mode II SIF as a function of (a) $a / W$ and (b) $\alpha$ for the CCP specimen 


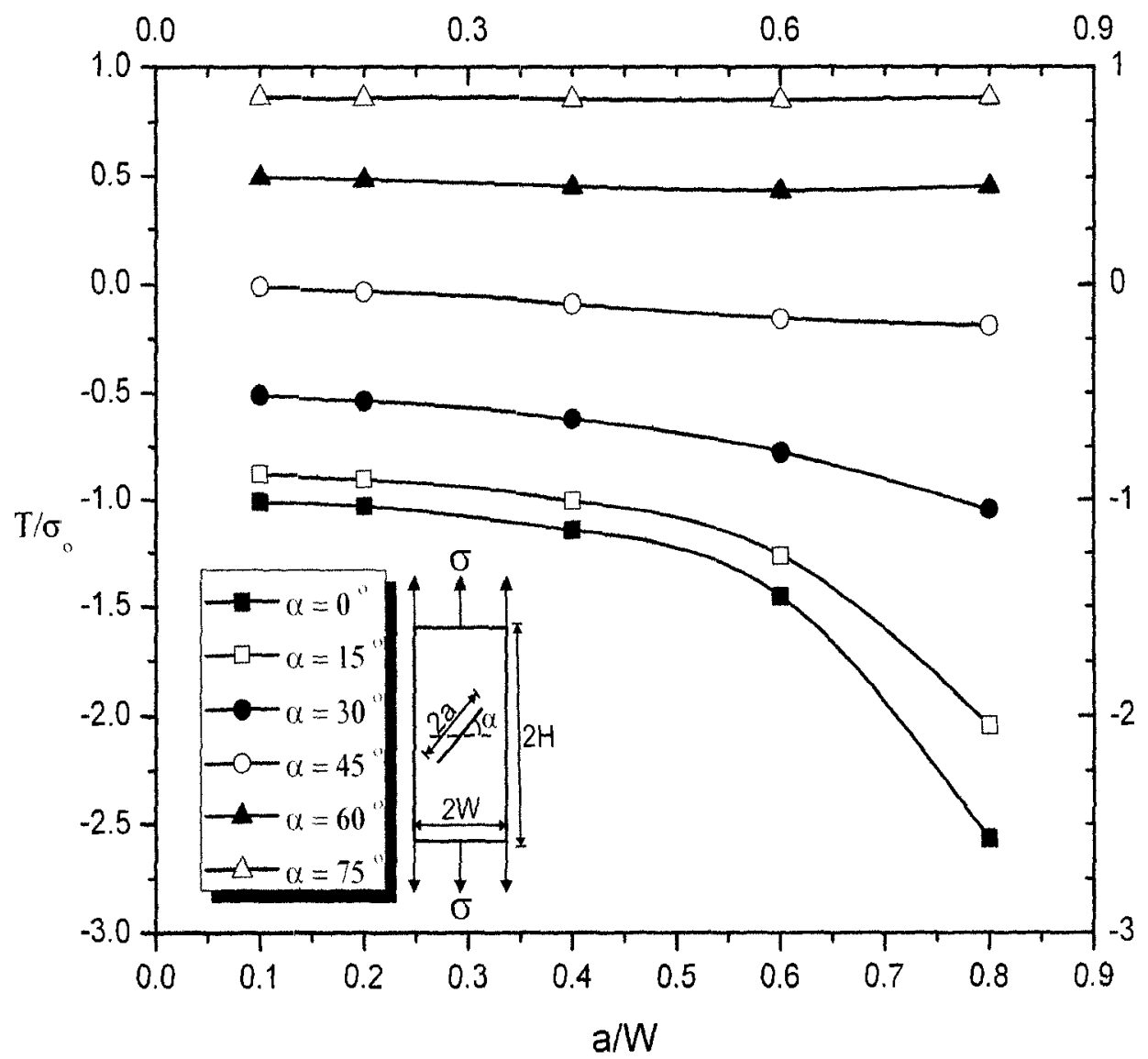

Figure 3.12: Normalized T-stress as a function of $a / W$ for the CCP specimen 


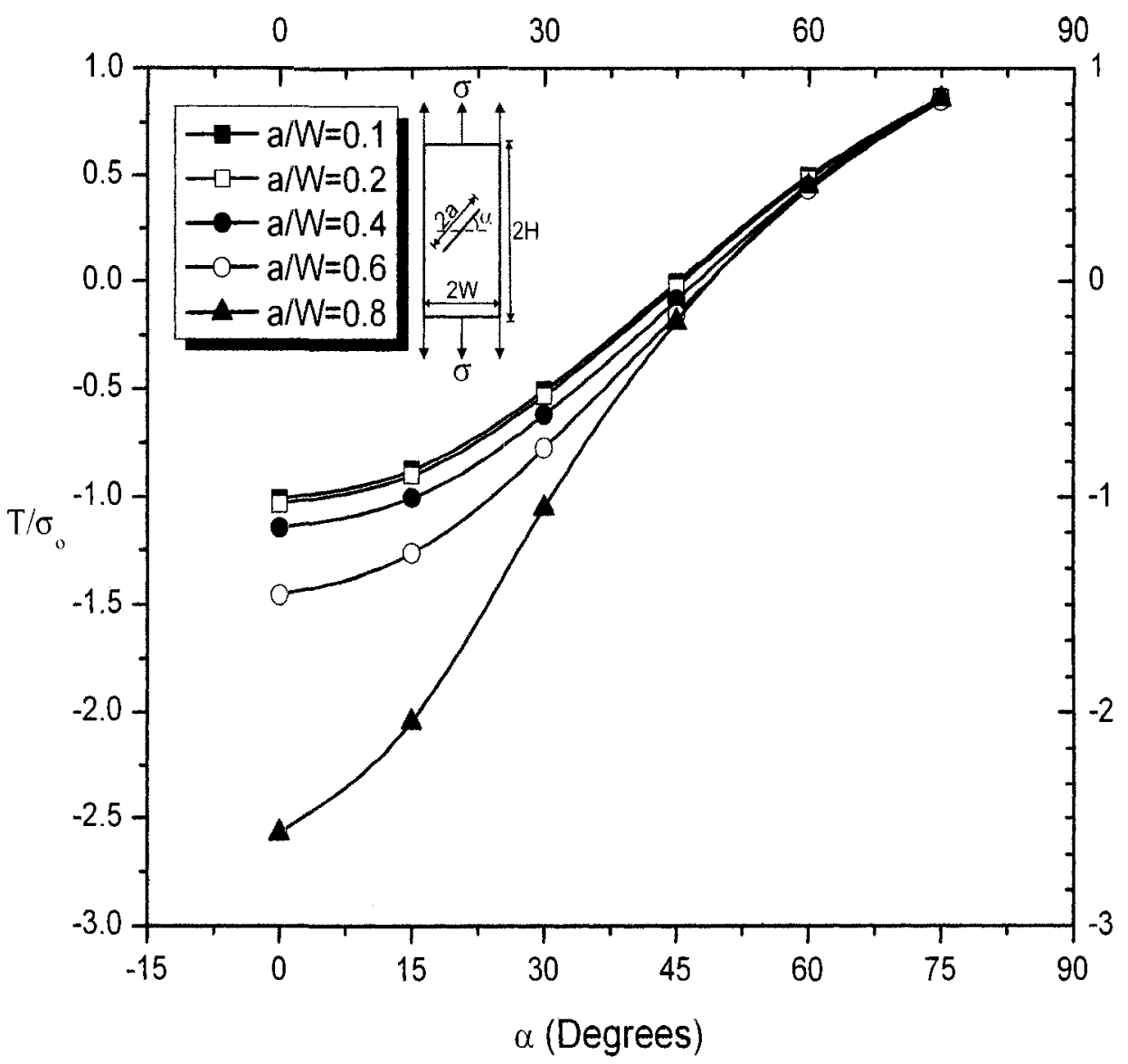

Figure 3.13: Normalized T-stress as a function of $\alpha$ for the CCP specimen 


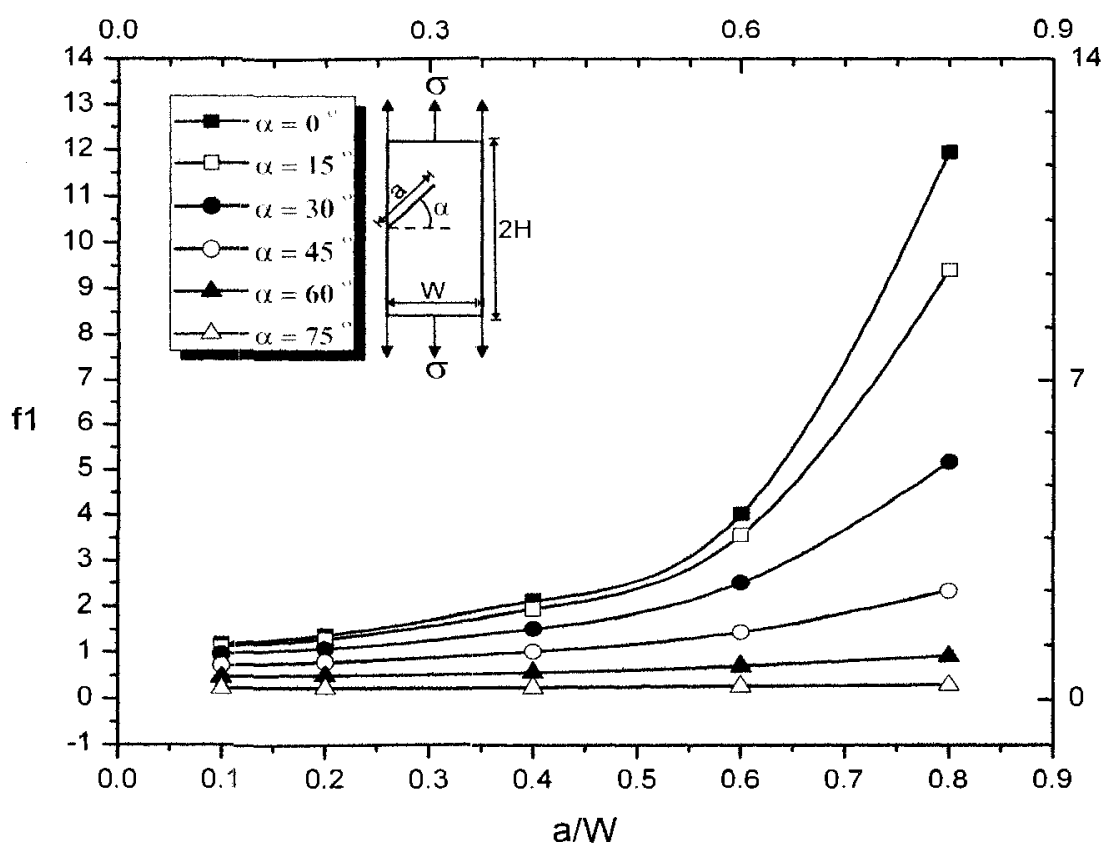

(a)

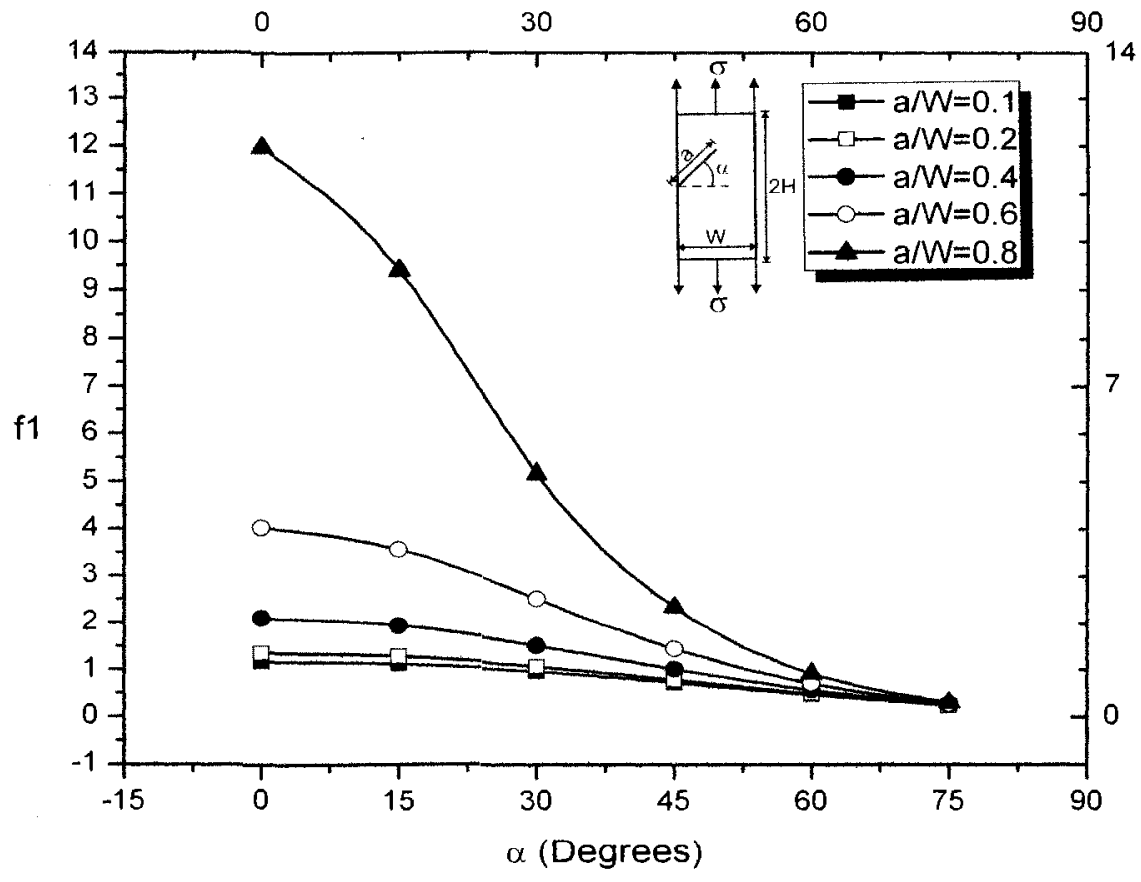

(b)

Figure 3.14: Normalized mode I SIF as a function of (a) $a / W$ and (b) $\alpha$ for the SECP specimen 


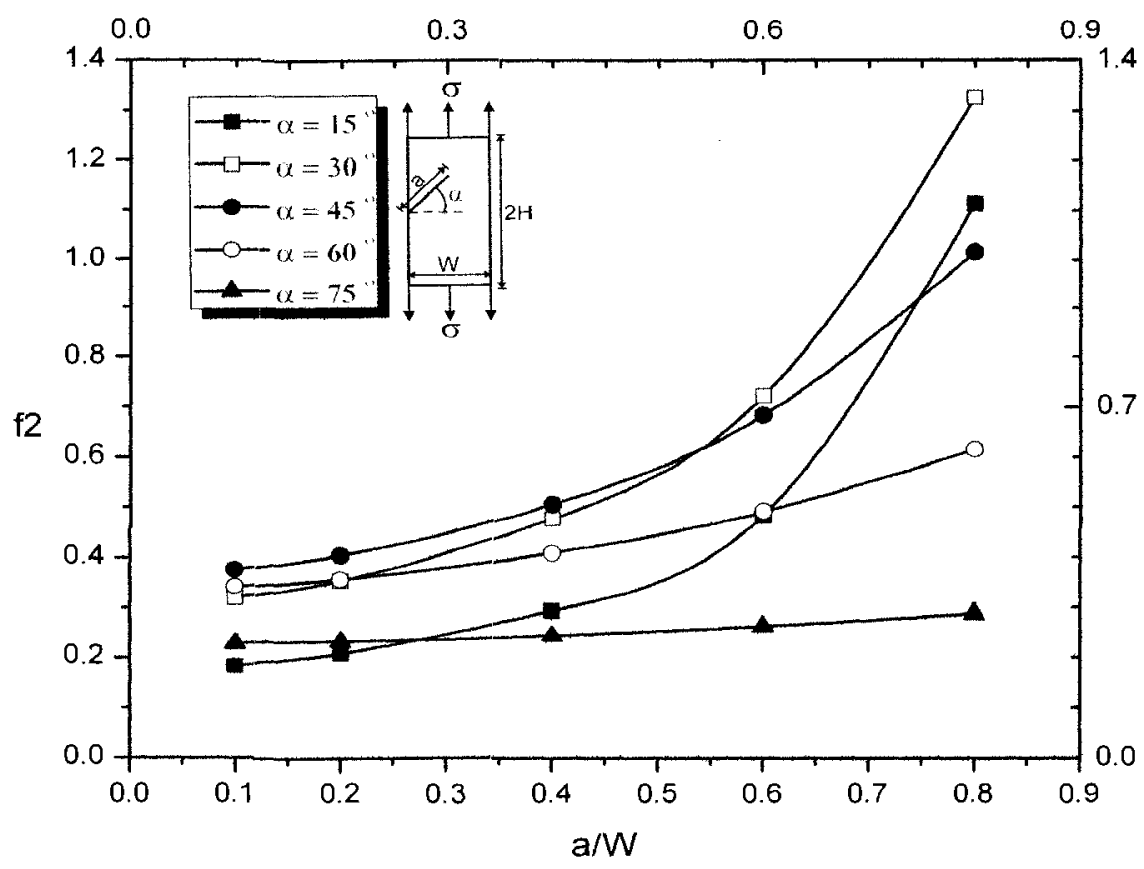

(a)

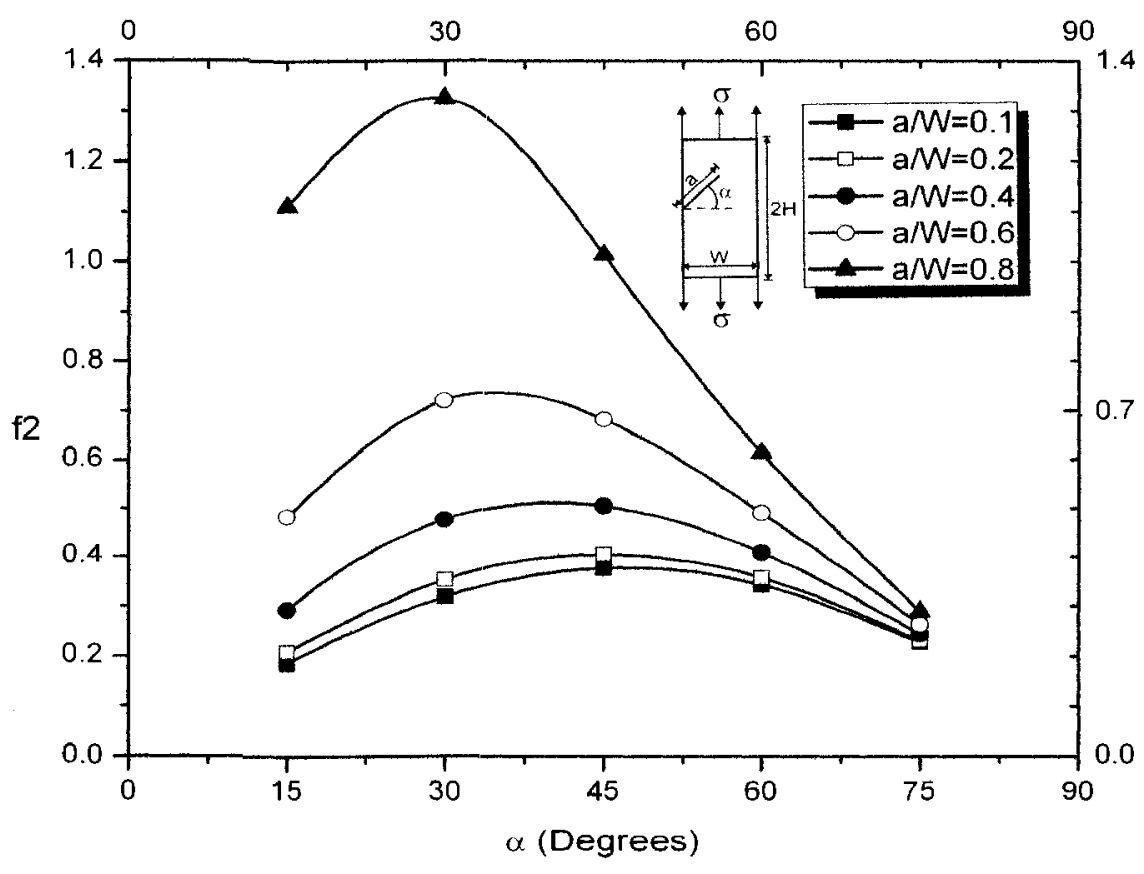

(b)

Figure 3.15: Normalized mode II SIF as a function of (a) $a / W$ (b) $\alpha$ for the SECP specimen 


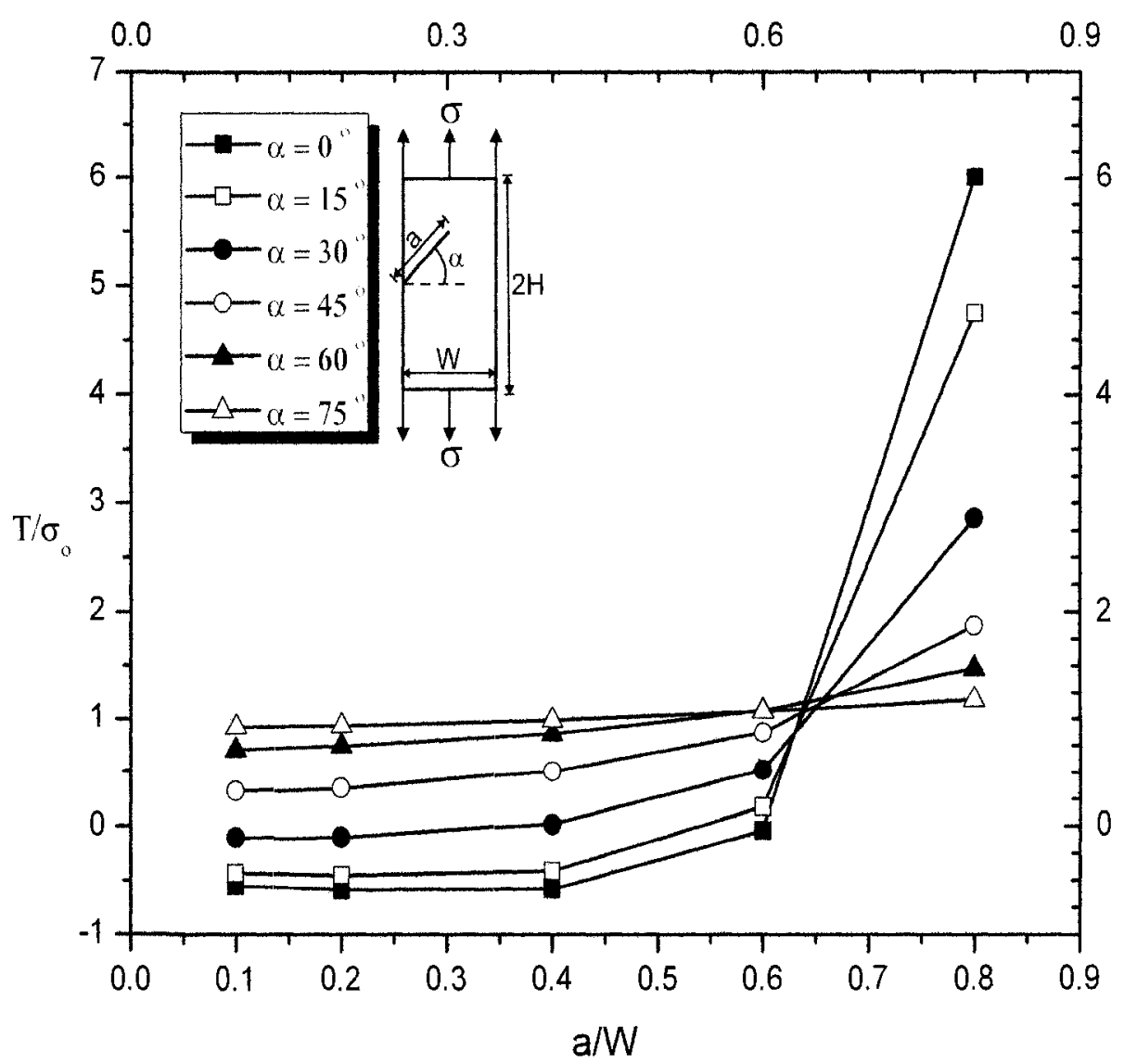

Figure 3.16: Normalized T-stress as a function of $a / W$ for the SECP specimen 


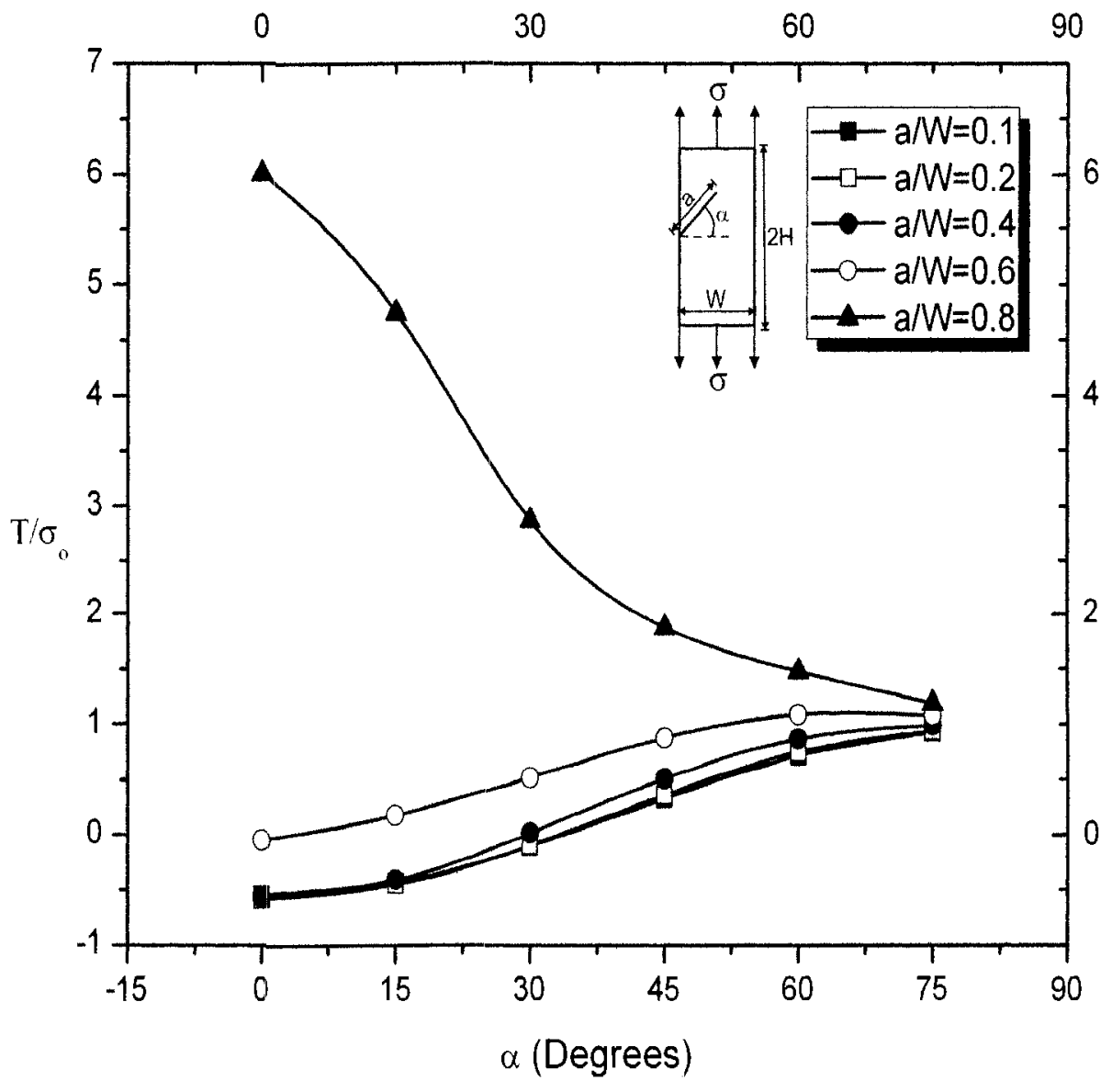

Figure 3.17: Normalized T-stress as a function of $\alpha$ for the SECP specimen 


\section{Chapter 4}

\section{Crack-Tip Plastic Zone Analysis Under Static Mixed-Mode Conditions}

In this chapter, the modeling, mapping, and analysis the crack-tip plastic zone as a result of a generalized stress-field influenced by T-stress will be conducted. The effects of the T-stress and the mode II to mode I stress intensity factor ratio on the size and shape of the plastic zone are investigated. The static plastic zone is then used to characterize constraint and material fracture resistance.

\subsection{The Plastic Zone}

The crack-tip plastic zone, also known as the crack-tip yielding zone and the damage zone, is the area within the close vicinity of the crack-tip that yields and plastically deforms as a result of a localized stress-field that is induced by the applied loading and is characterized by the geometry and orientation of the crack with respect to that of the specimen and associated applied loading. Therefore, the plastic zone for a generalized two-dimensional crack in a linear-elastic material can be mapped by modeling the effect of the crack-tip stress field on the yielding properties of an elasticperfectly-plastic material as characterized by the Von Mises yielding criterion.

\subsubsection{Modeling the Plastic Zone}

In two-parameter fracture mechanics using $\mathrm{T}$-stress as a constraint parameter, the stress field around the crack-tip (Fig. 2.3) of a two-dimensional crack embedded in an isotropic linear elastic material subjected to mixed mode I and mode II plain-strain loading conditions is given by the following expressions (Chapter 2):

$$
\sigma_{x x}=\frac{K_{I}}{\sqrt{2} \pi r} \cos \left(\frac{\theta}{2}\right)\left[1-\sin \left(\frac{\theta}{2}\right) \sin \left(\frac{3 \theta}{2}\right)\right]-\frac{K_{I}}{\sqrt{2 \pi r}} \sin \left(\frac{\theta}{2}\right)\left[2+\cos \left(\frac{\theta}{2}\right) \cos \left(\frac{3 \theta}{2}\right)\right]+T
$$




$$
\begin{aligned}
& \sigma_{y y}=\frac{K_{I}}{\sqrt{2 \pi r}} \cos \left(\frac{\theta}{2}\right)\left[1+\sin \left(\frac{\theta}{2}\right) \sin \left(\frac{3 \theta}{2}\right)\right]+\frac{K_{I}}{\sqrt{2 \pi r}} \sin \left(\frac{\theta}{2}\right) \cos \left(\frac{\theta}{2}\right) \cos \left(\frac{3 \theta}{2}\right) \\
& \sigma_{z z}=v\left(\sigma_{x x}+\sigma_{y y}\right)=2 v\left\{\frac{K_{I}}{\sqrt{2 \pi r}} \cos \left(\frac{\theta}{2}\right)-\frac{K_{I}}{\sqrt{2 \pi r}} \sin \left(\frac{\theta}{2}\right)\right\}+v T \\
& \tau_{x y}=\frac{K_{I}}{\sqrt{2 \pi r}} \sin \left(\frac{\theta}{2}\right) \cos \left(\frac{\theta}{2}\right) \cos \left(\frac{3 \theta}{2}\right)+\frac{K_{I I}}{\sqrt{2 \pi r}} \cos \left(\frac{\theta}{2}\right)\left[1-\sin \left(\frac{\theta}{2}\right) \sin \left(\frac{3 \theta}{2}\right)\right] \\
& \tau_{y z}, \tau_{z x}=0
\end{aligned}
$$

where, $(r, \theta)$ are the polar coordinates and $(x, y)$ are the Cartesian coordinates both with origins at the crack tip (Fig. 2.3). $K_{I}$ and $K_{I I}$ are the mode I and mode II stress intensity factors (SIF) respectively, $\sigma$ and $\tau$ are the normal and shear stress respectively, and $v$ is Poisson's ratio. The $T$ term in Equation 2.4a is the elastic T-stress, which under plane strain loading conditions induces a stress equivalent to $v T$ in the z-direction (Eqn 2.4c).

By definition, the plastic zone also known as the crack-tip-yielding zone is the region surrounding the crack tip that has yielded as a result of a localized stress field. In this case, the stress field is represented by Equation's 4.1a-e for plane strain conditions. The plastic zone around a crack-tip can therefore be determined using the Von Mises yield criterion below [Shigley, 2003]:

$\left[\left(\sigma_{x x}-\sigma_{y y}\right)^{2}+\left(\sigma_{y y}-\sigma_{z z}\right)^{2}+\left(\sigma_{z z}-\sigma_{x x}\right)^{2}+6\left(\tau_{x y}^{2}+\tau_{y z}^{2}+\tau_{z x}^{2}\right)\right] \leq 2 \sigma_{y}^{2}$

where, $\sigma_{y}$ is the material yield stress.

Similar to what was done by Anderson [2005] for single modes of fracture (mode I, II, and III), Kang \& Beom [2000] for a constrained ductile layer, and proposed by Gao et al [1985] for combined mode I and II loads (Chapter 2), the mixed-mode stress field 
(Eqn's 2.4a-e) is substituted into the Von Mises yield criterion (Eqn 2.7), and solved for the distance $r=r_{p}$ from the crack tip where the onset of yielding takes place. After comprehensive mathematical manipulations, which can be found in Appendix A, the solution for $r_{p}$ as a function of $\theta$ is given by the following formula:

$r_{p}(\theta)=\frac{1}{2 \pi}\left(\frac{K}{\sigma_{y}}\right)^{2}\left[\frac{1}{c^{*}}\left\{-[b] t_{y}-\sqrt{[b]^{2} t_{y}^{2}-[a] c^{*}}\right\}\right]^{2}$

where,

The term $r_{p}$ is the distance from the crack-tip where, for an applied stress field, the specimen begins to yield under static loading.

The net SIF $K=\sqrt{K_{I}^{2}+K_{I I}^{2}}$

Phase angle $\phi=\tan ^{-1} \frac{K_{I I}}{K_{I}}$

The net SIF $K$ represents the net combined effect of the mode I and mode II SIF's on the crack-tip, while $\phi$ represents the phase angle separating the mode I and mode II SIF's where $\tan \phi$ denotes the mode II to mode I SIF ratio. Note, the boundary conditions of $K$ and $\phi$ are $K=K_{I}$ at $\phi=0^{\circ}$ for a pure mode I loading condition, and $K=K_{I I}$ at $\phi=90^{\circ}$ for a pure mode II loading condition.

and

The normalized T-stress with respect to the yield stress $t_{y}=\frac{T}{\sigma_{y}}$ 
$c^{*}=[c] t_{y}^{2}-2$

$$
\begin{aligned}
{[a]=\left[\cos ^{2} \phi \cdot\left\{\frac{3}{2} \sin ^{2} \theta+V(\cos \theta+1)\right\}+\cos \phi \cdot \sin \phi \cdot\{\right.} & 2 \sin \theta \cdot(3 \cos \theta-V)\} \\
& \left.+\sin ^{2} \phi \cdot\left\{6-\frac{9}{2} \sin ^{2} \theta-V(\cos \theta-1)\right\}\right]
\end{aligned}
$$

$$
[b]=\left[\cos \phi \cdot\left\{\cos \frac{\theta}{2} \cdot\left(-3\left\{\cos ^{2} \frac{\theta}{2}-\cos ^{2} \theta\right\}+V\right)\right\}+\sin \phi \cdot\left\{\sin \frac{\theta}{2} \cdot\left(-3\left\{\cos ^{2} \frac{\theta}{2}+\cos ^{2} \theta\right\}-V\right)\right\}\right]
$$

$[c]=\left[\frac{1}{2}\{3+V\}\right]$

$V=(1-2 v)^{2}$

Note that,

$K_{I}=K \cos \phi$

$K_{I I}=K \sin \phi$

Looking at Equation 4.1, the plastic zone is explicitly a function of $K, \phi, T, \sigma_{y}$, and $v$.

\subsubsection{Comparison of the Plastic Zone Model with Special Cases in Literature}

To determine the validity of the plastic zone model derived above, Equation 4.1 is applied to a pure mode I situation and a mixed-mode situation both without T-stress, and 
the results compared with published solutions of Anderson [2005] and Gao et al [1985] respectively.

\section{Pure Mode I Case without T-stress}

Taking Equation 4.1 and setting the T-stress to zero gives:

$r_{p}(\theta)=\frac{1}{4 \pi}\left(\frac{K}{\sigma_{y}}\right)^{2}[a]$

substituting in Equations 4.5a and 4.8:

$$
\begin{aligned}
r_{p}(\theta)=\frac{1}{4 \pi \sigma_{y}^{2}}\left[K_{I}^{2}\left\{\frac{3}{2} \sin ^{2} \theta+V(\cos \theta+1)\right\}+K_{I} K_{I I}\{2 \sin \theta(3 \cos \theta-V)\}\right. & \\
+ & \left.+K_{I I}^{2}\left\{6-\frac{9}{2} \sin ^{2} \theta-V(\cos \theta-1)\right\}\right]
\end{aligned}
$$

setting $K_{I I}=0$ gives the plastic zone expression for a pure mode I loading condition:

$$
r_{p}(\theta)=\frac{1}{4 \pi}\left(\frac{K_{I}}{\sigma_{y}}\right)^{2}\left[\left\{\frac{3}{2} \sin ^{2} \theta+V(\cos \theta+1)\right\}\right]
$$

Equation 4.11 is identical to that derived by Anderson [2005] for pure mode I.

\section{Mixed Mode I and Mode II Case without T-stress}

Starting with Equation 4.10: a plastic zone expression for mixed mode I and mode II loading without $\mathrm{T}$-stress, and performing some trigonometric manipulations (Refer to Appendix A3), the relation on the following page is obtained: 


$$
\begin{aligned}
r_{p}(\theta)=\frac{1}{2 \pi \sigma_{y}^{2}}\left[K_{I}^{2}\left\{\cos ^{2} \frac{\theta}{2}\left(3 \sin ^{2} \frac{\theta}{2}+V\right)\right\}+K_{I} K_{I I}\{\sin \theta(3 \cos \theta-V)\}\right. & \\
& \left.+K_{I I}^{2}\left\{3+\sin ^{2} \frac{\theta}{2}\left(-9 \cos ^{2} \frac{\theta}{2}+V\right)\right\}\right]
\end{aligned}
$$

Equation 2.8 is the same formula used by Gao et al [1985] to determine the fatigue crack growth under combined mode I and mode II plane-strain loading conditions.

Being able to reduce Equation 4.1 into the exact formulas used and derived by both Gao et al [1985] and Anderson [2005] validates Equation 4.1 as well as the procedure employed to derive it (Appendix A).

\subsection{Plastic Zone Results}

The plastic zone was mapped and analyzed using the plastic zone model (Eqn 4.1) for a linear-elastic material with a Poisson's ratio of 0.3 ; a common value for steels. The plastic zone was analyzed for a wide range of mixed mode I and mode II loading conditions within the limits of the pure mode I and pure mode II loading conditions. The plastic zone was also analyzed for normalized T-stress values ranging from -1 to +1 .

\subsubsection{Mapping the Plastic Zone}

For various net SIF and normalized T-stress values, plastic zones were mapped around the crack-front from $\theta=-180^{\circ}$ to $\theta=180^{\circ}$ one degree at a time using the normalized form of Equation 4.1 below:

$$
\frac{r_{p}(\theta)}{h}=\frac{1}{2}\left[\frac{1}{c^{*}}\left\{-[b] t_{y}-\sqrt{[b]^{2} t_{y}^{2}-[a] c^{*}}\right\}\right]^{2}
$$


where the term $h$ is defined as:

$$
h=\frac{1}{\pi}\left(\frac{K}{\sigma_{y}}\right)^{2}
$$

The plastic zones were mapped for mode II to mode I SIF ratios (Eqn 4.3) ranging from $\phi=0^{\circ}$ to $\phi=90^{\circ}$ in increments of $10^{\circ}$ with normalized T-stress values ranging from $-1 \leq t_{y} \leq 1$ in increments of 0.25 . The SIF ratios of $\phi=0^{\circ}$ and $\phi=90^{\circ}$ represent the pure mode I and pure mode II cases respectively, and therefore all SIF ratios between these two limits represent mixed-mode conditions. Maps of the plastic zones can be viewed in Figures 4.1-4.10 with the corresponding data available in Tables 4.1-4.10. For convenience, the figures and tables are grouped according to their SIF ratios.

The range of the normalized T-stress (Eqn 4.3) was chosen to be within the limits of \pm 1 for the reason that none of the stresses (including the T-stress) within the region of the plastic zone can exceed the yield stress before yielding occurs in an elastic perfectly plastic material.

The plastic zones are mapped with the Cartesian coordinate system using the following polar to Cartesian conversions:

$$
\begin{aligned}
& x_{p}=r_{p}(\theta) \cdot \cos \theta \\
& y_{p}=r_{p}(\theta) \cdot \sin \theta
\end{aligned}
$$

Note that in Figures 4.1-4.10 the cracks emanate from the left-hand-side of the graphs with the crack-tips located at the origin in the center of the graph.

Looking at Figure 4.1-4.10, it is clear that the general shape and size of the plastic zone vary with both the mode II to mode I SIF ratio and $t_{y}$. The figures show that the 
plastic zones can be categorized into three distinct cases, namely $\phi=0^{\circ}, 0^{\circ}<\phi<90^{\circ}$, and $\phi=90^{\circ}$.

\subsubsection{Discussions}

The results displayed in Figure 4.1-4.10 and Tables 4.1-4.10 are discussed here.

\section{Pure Mode I $\left(\phi=0^{\circ}\right)$ Plastic Zones}

Figure 4.1 shows that the pure mode I plastic zones are generally shaped like a figure- 8 with a continuous boundary that is symmetric about the crack-front (i.e. the horizontal-axis). The figures also show that the plastic zone boundaries begin and end at the crack-tip.

The lobes of the plastic zone for $t_{y}=0$ are directionally neutral. However, the lobes become biased towards the crack-front when $t_{y}$ is positive and they become biased away from the crack-front when $t_{y}$ is negative. The directional bias of the lobes becomes more pronounced as $t_{y}$ becomes more positive or negative.

The size of the plastic zone varies with $t_{y}$ such that it decreases from $t_{y}=-1$ to $t_{y}=0$ and then increases from $t_{y}=0$ to $t_{y}=1$. In general, the plastic zones with negative $t_{y}$ values have an order of size twice as large as those with corresponding positive $t_{y}$ values.

\section{Mixed Mode I and Mode II $\left(0^{\circ}<\phi<90^{\circ}\right)$ Plastic Zones}

In general, from Figures 4.2-4.9, the plastic zones for mixed mode I and mode II loading conditions are shaped like a figure-8. The plastic zones with $t_{y}=0$ have continuous boundaries that begin and end at the crack-tip, while the plastic zones with $t_{y} \neq 0$ have a discontinuity in their boundary across the crack-front. 
As $t_{y}$ becomes more positive from $t_{y}=0$, the lobe(s) above the crack-front of the plastic zone become continuously less pronounced compared to those below the crackfront. The reverse is true for plastic zones with $t_{y}$ values that become more negative, i.e. the lobe(s) below the crack-front become continuously less pronounced compared to those above the crack-front. Also, as the $t_{y}$ values become either more positive or negative, the discontinuity in the boundary of the plastic zone gets larger.

The plastic zones for $\phi \geq 30^{\circ}$ at $t_{y}=-1$ and $t_{y}=1$ are almost entirely located above and below the crack-front respectively.

As the mode II to mode I SIF ratio increases, the plastic zones for all normalized T-stress values rotate clockwise about the crack-tip. Looking at the plastic zones for $\phi=0^{\circ}$ and $\phi=90^{\circ}$ at $t_{y}=0$, it is seen that the plastic zone goes through a clockwise rotation of a full $90^{\circ}$ about the crack-tip when going from a pure mode I loading condition to a pure mode II loading condition. Also, for a given $t_{y}$ as $\phi$ increases, the discontinuity in the boundary of the plastic zone increases as does the relative size difference between the lobes located below and above the crack-front.

The observations for mode I loading are consistent with finite element results reported in literature [Du \& Hancock, 1991; Subramanya; 2005].

\section{Pure Mode II $\left(\phi=90^{\circ}\right)$ Plastic Zones}

Referring to Figure 4.10 , the pure mode II plastic zones share all of the characteristics of the mixed mode I and mode II plastic zones as described in the subsection above. In addition to the shared characteristics, the plastic zones of normalized Tstress values with equal magnitudes but opposite signs share the same shape and size except that they are rotated $180^{\circ}$ from each other about the crack-tip. 


\subsubsection{Continuity of Plastic Zone Boundary}

From the plastic zone maps (Figs. 4.1-4.10), it was observed that a few of the maps have a continuous plastic zone boundary while the rest of the maps exhibit a discontinuity in the plastic zone boundary across the crack-front i.e. at $\theta= \pm 180^{\circ}$. To investigate the nature of the plastic zone boundary across the crack-front, expressions for the yield points at $\theta=-180^{\circ}$ and $\theta=+180^{\circ}$ were formulated in much the same way as Equation 4.3 and subtracted from each other. The resulting expression for the plastic zone boundary across the crack-front now dubbed the "dis/continuity" expression is displayed below:

$r_{p}(\theta=180)-r_{p}(\theta=-180)=\frac{-8}{\pi}\left(\frac{K_{I I}}{\sigma_{y}}\right)^{2} \frac{t_{y} W^{3 / 2}}{\left\{t_{y}^{2} W-1\right\}^{2}}$

where, $W=\left\{v^{2}-v+1\right\}$

The complete derivation of the dis/continuity expression can be found in Appendix B.

The nature of the plastic zone boundary across the crack-front has clearly been determined by the dis/continuity expression (Eqn 4.15). From the expression, it can be noted that the plastic zone boundary is continuous i.e. $r_{p}(\theta=180)-r_{p}(\theta=-180)=0$ if at least one of the following three conditions are met:

\section{1. $K_{I I}=0$}

Discontinuous plastic zone boundaries are possible only under pure mode II and mixed mode I and mode II loading conditions, while continuous plastic zone boundaries exist under pure mode I loading conditions. 
2. $t_{y}=0$

Discontinuous plastic zone boundaries are possible only when the T-stress is not zero, while continuous plastic zone boundaries exist when the T-stress is zero.

3. $W=0$

Solving Equation 4.19 using the quadratic formula shows that the term $\mathrm{W}$ can only be zero if the Poisson's ratio is a complex number, which is physically impossible as the Poisson's ratio is a material property and can only be represented by a real number.

Summarizing, the conditions necessary for a continuous plastic zone boundary are a pure mode I loading condition and/or a zero T-stress, while the conditions necessary for a discontinuous plastic zone boundary is a loading condition involving a mode II component (either wholly or partially) combined with a non-zero T-stress.

The results obtained from Equation 4.15 concerning the nature of the plastic zone boundary show excellent correspondence with the plastic zone maps obtained from Equation 4.12 further validating the dis/continuity expression.

\subsection{Maximum Plastic Zone Size Results}

For any given plastic zone, the maximum size $r_{p \max }$ is the most critical value characterizing that plastic zone. The maximum plastic zone size (PZS) was determined in its normalized form $r_{p \max } / h$ by first calculating the PZS one degree at a time from $\theta=-180^{\circ}$ to $\theta=180^{\circ}$ using Equation 4.12 and then manually identifying the maximum value. The values of the maximum PZS are available in Table 4.11.

To better understand the nature of the maximum PZS, its variation with both the normalized T-stress and the phase angle is explored in Figures 4.11-4.14. Note that, in Figures 4.11-4.14, the symbols represent the value of the maximum PZS as obtained from 
Equation 4.12 for those points and the curves linking the data points are lines of fit that display any trends present.

\section{Maximum Plastic Zone Size as a Function of Normalized T-stress}

The variation of $r_{p \max } / h$ as a function of the normalized T-stress can be observed in Figures 4.11-4.13. Each of Figures 4.11a-j show the maximum PZS variation for a given phase angle, while Figures 4.12 and 4.13 show the variation for all phase angles at $-1 \leq t_{y} \leq 1$ and $-0.75 \leq t_{y} \leq 0.75$ respectively. Figure 4.12 displays an overall view of the maximum PZS variation, whereas Figure 4.13 presents a detailed view of the maximum PZS variation thereby providing an improved means of examining the maximum PZS variation.

From Figures 4.11-4.13, the general trend of the maximum PZS versus normalized T-stress follows a squared-U-path. The maximum PZS drops sharply from $t_{y}=-1$ to $t_{y}=-0.75$ followed by a steady drop from $t_{y}=-0.75$ to $t_{y}=0$. The maximum PZS then shows a steady increase from $t_{y}=0$ to $t_{y}=0.75$ followed by a sharp increase from $t_{y}=0.75$ to $t_{y}=1$.

Figure 4.12 shows that for $0^{\circ} \leq \phi \leq 20^{\circ}$ the curve is skewed to the right, that is, the maximum PZS is smaller for the positive T-stress values compared to the corresponding negative T-stress values with equal magnitude, while for $30^{\circ} \leq \phi \leq 70^{\circ}$ the reverse is true, i.e. the maximum PZS is larger for the positive T-stress values compared to the corresponding negative T-stress values with equal magnitude. For $\phi=90^{\circ}$, the curve is perfectly symmetric about the zero T-stress with the maximum PZS being equal for T-stress values of equal magnitude but opposite sign. As $\phi$ increases from $0^{\circ}$ to $20^{\circ}$ the relative difference in maximum PZS between the positive a corresponding negative T-stresses decreases. The maximum PZS achieves almost perfect symmetry about the zero T-stress when $\phi=30^{\circ}$. As $\phi$ increases from $30^{\circ}$ to $50^{\circ}$ the relative difference in maximum PZS between the positive a corresponding negative T-stresses increases, and when $\phi$ increases from $60^{\circ}$ to $90^{\circ}$ the relative difference in maximum PZS between the 
positive a corresponding negative T-stresses again decreases until it reaches perfect equilibrium at $\phi=90^{\circ}$.

An important observation to note in Figure 4.12 is that not only does the maximum PSZ increase as the T-stress approaches the material yield stress value, but that the rate at which the maximum PZS increases with respect to the T-stress also increases as the T-stress approaches the yield stress value.

Referring to Figures 4.12 and 4.13, the magnitude of the maximum PZS increases with $\phi$ at a given T-stress. The largest and smallest values of the maximum PZS are associated with $\phi=90^{\circ}$ and $\phi=0^{\circ}$ respectively.

\section{Maximum Plastic Zone Size as a Function of Phase Angle}

The variation of the maximum PZS as a function of the phase angle is displayed in Figures 4.14a-e. The figures are such that the maximum PZS variations for T-stress values that are equal in magnitude but opposite in sign are grouped together in one graph. Curves with normalized T-stress values of equal magnitude but opposite signs are referred to as a "corresponding set".

In general, from Figure 4.14, the maximum PZS curves follow an S-shape, with the maximum PZS gradually increasing in value as the phase angle increases from $\phi=0^{\circ}$ to $\phi=90^{\circ}$. The maximum PZS value is larger for the negative T-stress compared to the corresponding positive T-stress when $0^{\circ} \leq \phi<30^{\circ}$, however for $30^{\circ}<\phi<90^{\circ}$ the trend reverses i.e. the positive T-stress has a larger maximum PZS than the corresponding negative T-stress. The maximum PZS curves for corresponding sets of T-stresses intersect at $\phi=30^{\circ}$ (where the switchover takes place) and then converge together at a single point when $\phi=90^{\circ}$. It is interesting to note that the above-mentioned characteristics are common to all corresponding T-stress sets. 
Referring again to Figure 4.14, the curves of corresponding T-stress sets exhibit the same order of magnitude which increases with the absolute value of the T-stress sets e.g. the order of magnitude is larger for the \pm 1 normalized $T$-stress set of curves than that of the \pm 0.75 normalized $T$-stress set of curves.

\section{Relating T-stress and Constraint using The Plastic Zone}

Under mode I loading conditions, it has been demonstrated that the PZS is an indicator of constraint as characterized by the T-stress, for example, under mode I loading, a positive $T$-stress results in a smaller plastic zone, which characterizes a higher level of constraint, and a negative T-stress results in a larger plastic zone which characterizes a low level of constraint [Smith et al, 2001]. Expanding the relationship between T-stress, constraint, and PZS to mixed-mode loading conditions and applying the results for the static plastic zone analysis above, it appears that the positive and negative T-stress only characterizes a high and low level of constraint respectively provided that $\phi<30^{\circ}$, while for $\phi>30^{\circ}$ the reverse is true, that is, the positive T-stress characterizes a low level of constraint and the negative T-stress characterizes a high level of constraint. Note that the statements concerning T-stress, constraint, and the plastic zone for mixed-mode loading requires confirmation through further investigation.

\subsection{Characterizing Fracture Resistance}

It has been determined that the PZS under static loading is a good parameter for characterizing material fracture resistance in that, a material with a larger PZS at fracture has undergone greater plastic deformation at the crack-tip and as such exhibits a larger fracture resistance [Kang \& Beom, 2000]. Currently, the most common means of characterizing material fracture resistance is the fracture toughness as defined by the critical SIF, that is, the SIF at which a material will fracture. However, knowing that the crack-tip PZS is an indicator of a material's fracture resistance and that according to Equation 4.1 the plastic zone size is a function of both SIF and T-stress, fracture resistance cannot accurately be characterized by the SIF alone; the T-stress effect must also be considered. 
Since the PZS at fracture is an indication of the degree of a material's fracture resistance, it is proposed that PZS as a result of mixed-mode loading and T-stress could be used to characterize the fracture resistance by means of a critical maximum PZS derived from Equation 4.1 and defined below:

$\left(r_{p \max }\right)_{c}=\frac{1}{2 \pi}\left[\left(\frac{K}{\sigma_{y}}\right)\left(\frac{1}{c^{*}}\left\{-[b] t_{y}-\sqrt{[b]^{2} t_{y}^{2}-[a] c^{*}}\right\}\right)_{\max }\right]_{c}^{2}$

where the subscript $c$ denotes the critical value.

From Equation 4.17, characterizing the fracture resistance with the SIF and the Tstress is not as simple as defining a critical SIF and a critical T-stress for the reason that it is the combined effect of both the SIF and the T-stress that determines the crack-tip PZS and therefore the material fracture resistance.

The fracture resistance, characterized by Equation 4.17 , is the result of the combined effect of SIF and T-stress such that numerous combinations of SIF and T-stress will produce the same result. Furthermore, Equation 4.17 can be applied to mixed mode I and mode II loading conditions such that, if the fracture toughness/critical effective SIF is known for a given material, then Equation 4.17 can be used to determine if and how the T-stress may affect it.

Equation 4.17 is a proposed means of illustrating how fracture toughness may be influenced by $\mathrm{T}$-stress and it has not been validated through experimental results; it is a theory that is recommended for future study.

\section{Influence of T-stress and Phase Angle on Fracture Resistance}

The idea behind Equation 4.17 is to calculate the maximum critical PZS for a critical SIF/fracture toughness and compare it to the critical PZS calculated from the combined effect of the critical SIF and accompanying T-stress for a given test specimen, thereby determining if and how the T-stress may affect fracture toughness. 
The normalized critical maximum PZS for a linear elastic material of Poisson's ratio 0.3 can be obtained from Figures 4.11-4.14.

From Figures 4.12 and 4.13, it can be observed that for a given phase angle and SIF, the critical maximum PZS increases with the absolute value of T-stress. Therefore, for a given SIF and phase angle, a larger absolute T-stress will result in a larger PZS and therefore a larger fracture resistance.

From Figure 4.14, for a given phase angle, the critical maximum PZS for a given SIF and therefore the fracture resistance for a negative T-stress compared to that of a positive T-stress is:

a) Larger only if the mode II to mode I SIF ratio of less than $\tan 30^{\circ}$.

b) Smaller only if the mode II to mode I SIF ratio is between $\tan 30^{\circ}$ and $\tan 90^{\circ}$.

c) Equal only when the mode II to mode I SIF ratio is equal to $\tan 30^{\circ}$ or $\tan 90^{\circ}$.

The observations in point a) above correlate well with experimental results [Larsson \& Carlsson, 1983; Du \& Hancock, 1991; Ayatollahi et al, 1998].

Also from Figure 4.14, for a given T-stress, the maximum critical PZS for a given SIF increases with the phase angle implying that the fracture resistance for a given critical SIF increases for loading conditions with a predominantly larger mode II component.

Knowing the trend of fracture resistance with respect to phase angle and T-stress can help establish the conditions under which a material will fracture, and therefore also provide an accurate means of characterizing material fracture toughness using mixedmode SIF's and T-stress. 


\subsection{Conclusion}

The crack-tip plastic zone was analyzed for various mixed mode I and mode II loading conditions influenced by $\mathrm{T}$-stress. It was determined that the size and orientation of the plastic zone varied greatly with the mode II to mode I SIF ratio as well as the Tstress, demonstrating that the T-stress is a important parameter to consider in addition to the SIF when analyzing fracture.

By expanding the relationship between T-stress, constraint, and the plastic zone to mixed-mode loading conditions, it is observed that the characterization of constraint (with respect to the PZS) using T-stress depends on the value of the mode II to mode I SIF ratio.

The plastic zone was also used to propose a relation for fracture resistance that considers the T-stress effect under mixed-mode loading conditions. The maximum PZS was used as a measure for determining the fracture resistance under critical loading conditions, where a material with a larger crack-tip plastic zone at fracture exhibited a greater resistance to fracture. 
Table 4.1: Data for mapping the normalized plastic zone $r_{p}(\theta) / h$ for $\phi=0^{\circ}$

\begin{tabular}{cccccccccc}
\hline Theta & \multicolumn{1}{c}{ Normalized T-stress, $t_{y}$} \\
\cline { 2 - 10 }$\theta$ & -1 & -0.75 & -0.5 & -0.25 & 0 & 0.25 & 0.5 & 0.75 & 1 \\
\hline$-180^{\circ}$ & 0 & 0 & 0 & 0 & 0 & 0 & 0 & 0 & 0 \\
$-165^{\circ}$ & 0.0085 & 0.0105 & 0.0135 & 0.0183 & 0.0265 & 0.0424 & 0.0804 & 0.2161 & 1.8772 \\
$-150^{\circ}$ & 0.0484 & 0.0529 & 0.0612 & 0.0751 & 0.0991 & 0.1447 & 0.2493 & 0.6017 & 4.6017 \\
$-135^{\circ}$ & 0.2166 & 0.1731 & 0.1647 & 0.1734 & 0.1992 & 0.2533 & 0.3741 & 0.7428 & 4.1547 \\
$-120^{\circ}$ & 1.1461 & 0.4888 & 0.3544 & 0.3086 & 0.3013 & 0.3254 & 0.3976 & 0.6014 & 1.7955 \\
$-105^{\circ}$ & 5.3755 & 1.1511 & 0.6332 & 0.4567 & 0.3795 & 0.3490 & 0.3532 & 0.4053 & 0.6076 \\
$-90^{\circ}$ & 13.850 & 2.0380 & 0.9168 & 0.5695 & 0.4150 & 0.3346 & 0.2917 & 0.2737 & 0.2820 \\
$-75^{\circ}$ & 20.278 & 2.5768 & 1.0442 & 0.5948 & 0.4002 & 0.2980 & 0.2382 & 0.2014 & 0.1791 \\
$-60^{\circ}$ & 18.375 & 2.2905 & 0.9140 & 0.5136 & 0.3413 & 0.2509 & 0.1978 & 0.1647 & 0.1437 \\
$-45^{\circ}$ & 10.140 & 1.4001 & 0.6035 & 0.3622 & 0.2558 & 0.1999 & 0.1683 & 0.1514 & 0.1463 \\
$-30^{\circ}$ & 2.9897 & 0.5751 & 0.3006 & 0.2091 & 0.1684 & 0.1501 & 0.1465 & 0.1597 & 0.2151 \\
$-15^{\circ}$ & 0.4708 & 0.1826 & 0.1277 & 0.1085 & 0.1038 & 0.1098 & 0.1309 & 0.1909 & 0.5185 \\
$0^{\circ}$ & 0.1633 & 0.0965 & 0.0798 & 0.0760 & 0.0800 & 0.0932 & 0.1246 & 0.2148 & 0.8889 \\
$15^{\circ}$ & 0.4708 & 0.1826 & 0.1277 & 0.1085 & 0.1038 & 0.1098 & 0.1309 & 0.1909 & 0.5185 \\
$30^{\circ}$ & 2.9897 & 0.5751 & 0.3006 & 0.2091 & 0.1684 & 0.1501 & 0.1465 & 0.1597 & 0.2151 \\
$45^{\circ}$ & 10.140 & 1.4001 & 0.6035 & 0.3622 & 0.2558 & 0.1999 & 0.1683 & 0.1514 & 0.1463 \\
$60^{\circ}$ & 18.375 & 2.2905 & 0.9140 & 0.5136 & 0.3413 & 0.2509 & 0.1978 & 0.1647 & 0.1437 \\
$75^{\circ}$ & 20.278 & 2.5768 & 1.0442 & 0.5948 & 0.4002 & 0.2980 & 0.2382 & 0.2014 & 0.1791 \\
$90^{\circ}$ & 13.850 & 2.0380 & 0.9168 & 0.5695 & 0.4150 & 0.3346 & 0.2917 & 0.2737 & 0.2820 \\
$105^{\circ}$ & 5.3755 & 1.1511 & 0.6332 & 0.4567 & 0.3795 & 0.3490 & 0.3532 & 0.4053 & 0.6076 \\
$120^{\circ}$ & 1.1461 & 0.4888 & 0.3544 & 0.3086 & 0.3013 & 0.3254 & 0.3976 & 0.6014 & 1.7955 \\
$135^{\circ}$ & 0.2166 & 0.1731 & 0.1647 & 0.1734 & 0.1992 & 0.2533 & 0.3741 & 0.7428 & 4.1547 \\
$150^{\circ}$ & 0.0484 & 0.0529 & 0.0612 & 0.0751 & 0.0991 & 0.1447 & 0.2493 & 0.6017 & 4.6017 \\
$165^{\circ}$ & 0.0085 & 0.0105 & 0.0135 & 0.0183 & 0.0265 & 0.0424 & 0.0804 & 0.2161 & 1.8772 \\
$180^{\circ}$ & 0 & 0 & 0 & 0 & 0 & 0 & 0 & 0 & 0 \\
\hline & & & & & & & & & \\
\hline
\end{tabular}


Table 4.2: Data for mapping the normalized plastic zone $r_{p}(\theta) / h$ for $\phi=10^{\circ}$

\begin{tabular}{|c|c|c|c|c|c|c|c|c|c|}
\hline \multirow{2}{*}{$\begin{array}{c}\text { Theta } \\
\boldsymbol{\theta}\end{array}$} & \multicolumn{9}{|c|}{ Normalized T-stress, $t_{y}$} \\
\hline & -1 & -0.75 & -0.5 & -0.25 & 0 & 0.25 & 0.5 & 0.75 & 1 \\
\hline$-180^{\circ}$ & 0.0132 & 0.0170 & 0.0226 & 0.0317 & 0.0474 & 0.0785 & 0.1542 & 0.4291 & 3.8660 \\
\hline$-165^{\circ}$ & 0.0421 & 0.0530 & 0.0691 & 0.0946 & 0.1385 & 0.2243 & 0.4308 & 1.1721 & 10.321 \\
\hline$-150^{\circ}$ & 0.1059 & 0.1216 & 0.1463 & 0.1857 & 0.2528 & 0.3810 & 0.6788 & 1.7032 & 13.685 \\
\hline$-135^{\circ}$ & 0.2855 & 0.2612 & 0.2683 & 0.2990 & 0.3613 & 0.4832 & 0.7557 & 1.6190 & 10.368 \\
\hline$-120^{\circ}$ & 0.9817 & 0.5524 & 0.4472 & 0.4197 & 0.4366 & 0.5027 & 0.6620 & 1.1177 & 4.4033 \\
\hline$-105^{\circ}$ & 3.8172 & 1.0729 & 0.6624 & 0.5174 & 0.4605 & 0.4535 & 0.4971 & 0.6402 & 1.2597 \\
\hline$-90^{\circ}$ & 9.6787 & 1.6711 & 0.8261 & 0.5516 & 0.4287 & 0.3686 & 0.3454 & 0.3562 & 0.4305 \\
\hline$-75^{\circ}$ & 13.487 & 1.8847 & 0.8192 & 0.4950 & 0.3517 & 0.2766 & 0.2345 & 0.2126 & 0.2080 \\
\hline$-60^{\circ}$ & 10.813 & 1.4523 & 0.6138 & 0.3624 & 0.2521 & 0.1941 & 0.1608 & 0.1417 & 0.1333 \\
\hline$-45^{\circ}$ & 4.6724 & 0.7208 & 0.3340 & 0.2124 & 0.1580 & 0.1301 & 0.1161 & 0.1122 & 0.1212 \\
\hline$-30^{\circ}$ & 0.8860 & 0.2352 & 0.1419 & 0.1091 & 0.0958 & 0.0931 & 0.1005 & 0.1265 & 0.2351 \\
\hline$-15^{\circ}$ & 0.1855 & 0.1046 & 0.0847 & 0.0796 & 0.0828 & 0.0954 & 0.1257 & 0.2125 & 0.8388 \\
\hline $0^{\circ}$ & 0.2954 & 0.1604 & 0.1279 & 0.1189 & 0.1226 & 0.1399 & 0.1824 & 0.3035 & 1.1538 \\
\hline $15^{\circ}$ & 1.4806 & 0.4464 & 0.2834 & 0.2256 & 0.2040 & 0.2042 & 0.2281 & 0.3020 & 0.6374 \\
\hline $30^{\circ}$ & 6.7324 & 1.1727 & 0.5825 & 0.3904 & 0.3043 & 0.2625 & 0.2468 & 0.2558 & 0.3119 \\
\hline $45^{\circ}$ & 17.294 & 2.3068 & 0.9700 & 0.5703 & 0.3952 & 0.3030 & 0.2500 & 0.2193 & 0.2047 \\
\hline $60^{\circ}$ & 26.917 & 3.2512 & 1.2637 & 0.6935 & 0.4505 & 0.3239 & 0.2494 & 0.2022 & 0.1710 \\
\hline $75^{\circ}$ & 27.390 & 3.2980 & 1.2783 & 0.6998 & 0.4535 & 0.3253 & 0.2498 & 0.2020 & 0.1703 \\
\hline $90^{\circ}$ & 18.294 & 2.4067 & 1.0017 & 0.5838 & 0.4013 & 0.3053 & 0.2497 & 0.2168 & 0.1996 \\
\hline $105^{\circ}$ & 7.4708 & 1.2469 & 0.6050 & 0.3984 & 0.3058 & 0.2598 & 0.2400 & 0.2430 & 0.2839 \\
\hline $120^{\circ}$ & 1.6495 & 0.4535 & 0.2775 & 0.2155 & 0.1908 & 0.1869 & 0.2037 & 0.2600 & 0.5004 \\
\hline $135^{\circ}$ & 0.2154 & 0.1143 & 0.0903 & 0.0833 & 0.0855 & 0.0970 & 0.1257 & 0.2071 & 0.7693 \\
\hline $150^{\circ}$ & 0.0208 & 0.01575 & 0.0146 & 0.0151 & 0.0170 & 0.0213 & 0.0308 & 0.0596 & 0.3167 \\
\hline $165^{\circ}$ & 0.2687 & 0.0294 & 0.0104 & 0.0052 & 0.0031 & 0.0021 & 0.0014 & 0.0011 & 0.0008 \\
\hline $180^{\circ}$ & 3.8660 & 0.4291 & 0.1542 & 0.0785 & 0.0474 & 0.0317 & 0.0226 & 0.0170 & 0.0132 \\
\hline
\end{tabular}


Table 4.3: Data for mapping the normalized plastic zone $r_{p}(\theta) / h$ for $\phi=20^{\circ}$

\begin{tabular}{|c|c|c|c|c|c|c|c|c|c|}
\hline \multirow{2}{*}{$\begin{array}{c}\text { Theta } \\
\boldsymbol{\theta}\end{array}$} & \multicolumn{9}{|c|}{ Normalized T-stress, $t_{y}$} \\
\hline & -1 & & & -0.25 & 0 & 0.25 & & 0.75 & 1 \\
\hline & 0.0518 & 0666 & 0886 & & & & 0.5988 & 1.6629 & 14.951 \\
\hline$-165^{\circ}$ & 0985 & 1241 & 1622 & 2224 & 3263 & 5297 & & & 24.521 \\
\hline & 1817 & 24 & 3 & & .4602 & & & & .422 \\
\hline$-135^{\circ}$ & 7 & 6 & 55 & 15 & 25 & & & & \\
\hline-120 & 269 & & & & 58 & 988 & 0.9 & & \\
\hline & & & & & & & & & \\
\hline$-90^{\circ}$ & 5 & & & & 0.4 & 56 & 0.4 & 0.4722 & 0.7102 \\
\hline$-75^{\circ}$ & & & & & & & & & \\
\hline$-60^{\circ}$ & 31 & & & 561 & 0.19 & 0.1 & 0.1 & & 0.1671 \\
\hline$-45^{\circ}$ & & & & & & & & & \\
\hline$-30^{\circ}$ & & & & & & & & & \\
\hline$-15^{0}$ & & & & & & & & & \\
\hline $0^{\circ}$ & 0.7 & & & & 51 & 36 & 0.3 & & \\
\hline $15^{\circ}$ & & & & & & & & & \\
\hline $30^{\circ}$ & & & & & & & 0.3 & & \\
\hline $45^{\circ}$ & & & & & & & & & \\
\hline $60^{\circ}$ & & & & & & & 0.3 & & 0.2060 \\
\hline $75^{\circ}$ & 33.92 & 3.96 & 1.49 & 0.7984 & 0.50 & 0.3536 & 0.2650 & 0.2086 & \\
\hline $90^{\circ}$ & & & & 9 & & 0.2 & 0.2 & & \\
\hline & 10.0 & & & & & & 0.1 & & \\
\hline $120^{\circ}$ & 2.99 & 0.49 & & & & & 0.0 & & 074 \\
\hline $135^{\circ}$ & & & & & & & & & 0.0314 \\
\hline $150^{\circ}$ & 1.3598 & & & & & & & & \\
\hline $165^{\circ}$ & & & & & & & 0.0 & & 0.0209 \\
\hline $180^{\circ}$ & 14.951 & & & 3056 & & & 0.0886 & 0.0666 & 0.0518 \\
\hline
\end{tabular}


Table 4.4: Data for mapping the normalized plastic zone $r_{p}(\theta) / h$ for $\phi=30^{\circ}$

\begin{tabular}{|c|c|c|c|c|c|c|c|c|c|}
\hline \multirow{2}{*}{$\begin{array}{c}\text { Theta } \\
\theta\end{array}$} & \multicolumn{9}{|c|}{ Normalized T-stress, $t_{y}$} \\
\hline & -1 & -0.75 & -0.5 & -0.25 & 0 & 0.25 & 0.5 & 0.75 & 1 \\
\hline$-180^{\circ}$ & 0.1107 & 0.1422 & 0.1893 & 0.2644 & 0.3950 & 0.6529 & 1.2796 & 3.5539 & 31.955 \\
\hline$-165^{\circ}$ & 0.1703 & 0.2149 & 0.2812 & 0.3861 & 0.5670 & 0.9215 & 1.7755 & 4.8468 & 42.823 \\
\hline$-150^{\circ}$ & 0.2660 & 0.3139 & 0.3866 & 0.5012 & 0.6962 & 1.0702 & 1.9472 & 5.0018 & 41.331 \\
\hline$-135^{\circ}$ & 0.4540 & 0.4602 & 0.5046 & 0.5924 & 0.7501 & 1.0511 & 1.7314 & 3.9605 & 28.104 \\
\hline$-120^{\circ}$ & 0.8972 & 0.6723 & 0.6193 & 0.6365 & 0.7162 & 0.8918 & 1.2863 & 2.4716 & 12.966 \\
\hline$-105^{\circ}$ & 1.9894 & 0.9178 & 0.6860 & 0.6098 & 0.6057 & 0.6657 & 0.8303 & 1.2946 & 4.1811 \\
\hline$-90^{\circ}$ & 3.6428 & 1.0363 & 0.6428 & 0.5038 & 0.4497 & 0.4440 & 0.4884 & 0.6320 & 1.2585 \\
\hline$-75^{\circ}$ & 3.7718 & 0.8440 & 0.4735 & 0.3463 & 0.2913 & 0.2711 & 0.2782 & 0.3256 & 0.5100 \\
\hline$-60^{\circ}$ & 1.7980 & 0.4504 & 0.2653 & 0.2007 & 0.1738 & 0.1665 & 0.1767 & 0.2171 & 0.3808 \\
\hline$-45^{\circ}$ & 0.4232 & 0.1953 & 0.1460 & 0.1298 & 0.1289 & 0.1417 & 0.1767 & 0.2756 & 0.8901 \\
\hline$-30^{\circ}$ & 0.2340 & 0.1671 & 0.1503 & 0.1519 & 0.1684 & 0.2066 & 0.2930 & 0.5499 & 2.7482 \\
\hline$-15^{\circ}$ & 0.4485 & 0.2996 & 0.2613 & 0.2582 & 0.2809 & 0.3381 & 0.4690 & 0.8532 & 3.9894 \\
\hline $0^{\circ}$ & 1.5000 & 0.6743 & 0.4989 & 0.4405 & 0.4350 & 0.4754 & 0.5889 & 0.9090 & 2.8605 \\
\hline $15^{\circ}$ & 5.8742 & 1.498 & 0.8889 & 0.6759 & 0.5877 & 0.5656 & 0.6034 & 0.7469 & 1.3334 \\
\hline $30^{\circ}$ & 17.323 & 2.8690 & 1.3860 & 0.9097 & 0.6962 & 0.5897 & 0.5431 & 0.5473 & 0.6345 \\
\hline $45^{\circ}$ & 33.060 & 4.3576 & 1.8163 & 1.0598 & 0.7294 & 0.5555 & 0.4548 & 0.3955 & 0.3649 \\
\hline $60^{\circ}$ & 42.727 & 5.0777 & 1.9458 & 1.0541 & 0.6762 & 0.4801 & 0.3649 & 0.2917 & 0.2427 \\
\hline $75^{\circ}$ & 39.141 & 4.4983 & 1.6720 & 0.8801 & 0.5491 & 0.3791 & 0.2800 & 0.2171 & 0.1747 \\
\hline $90^{\circ}$ & 25.956 & 3.0185 & 1.1344 & 0.6034 & 0.3804 & 0.2653 & 0.1980 & 0.1552 & 0.1264 \\
\hline $105^{\circ}$ & 12.846 & 1.5495 & 0.6015 & 0.3298 & 0.2140 & 0.1537 & 0.1182 & 0.0957 & 0.0808 \\
\hline $120^{\circ}$ & 5.6408 & 0.6800 & 0.2638 & 0.1446 & 0.0938 & 0.0673 & 0.0517 & 0.0419 & 0.0353 \\
\hline $135^{\circ}$ & 4.0992 & 0.4580 & 0.1657 & 0.0849 & 0.0516 & 0.0347 & 0.0250 & 0.0188 & 0.0147 \\
\hline $150^{\circ}$ & 7.5648 & 0.8539 & 0.3120 & 0.1615 & 0.0991 & 0.0673 & 0.0489 & 0.0373 & 0.0294 \\
\hline $165^{\circ}$ & 17.419 & 1.9575 & 0.7121 & 0.3671 & 0.2243 & 0.1517 & 0.1097 & 0.0833 & 0.0655 \\
\hline $180^{\circ}$ & 31.955 & 3.5539 & 1.2796 & 0.6529 & 0.3950 & 0.2644 & 0.1893 & 0.1422 & 0.1107 \\
\hline
\end{tabular}


Table 4.5: Data for mapping the normalized plastic zone $r_{p}(\theta) / h$ for $\phi=40^{\circ}$

\begin{tabular}{|c|c|c|c|c|c|c|c|c|c|}
\hline \multirow{2}{*}{$\begin{array}{c}\text { Theta } \\
\theta\end{array}$} & \multicolumn{9}{|c|}{ Normalized T-stress, $t_{y}$} \\
\hline & -1 & -0.75 & -0.5 & -0.25 & 0 & 0.25 & 0.5 & 0.75 & 1 \\
\hline$-180^{\circ}$ & 0.1827 & 0.2348 & 3126 & 4367 & 0.6525 & 9789 & 2.1150 & .8753 & 52.841 \\
\hline$-165^{\circ}$ & 485 & 140 & 0.4113 & 5653 & 0.8311 & 3521 & 2.6077 & & 63.027 \\
\hline$-150^{\circ}$ & & 0 & & 6671 & 9316 & 4395 & & & \\
\hline$-135^{\circ}$ & & 32 & 70 & & 0.92 & 204 & & & \\
\hline$-120^{\circ}$ & & & 6733 & 43 & .8263 & 1.0577 & & & \\
\hline$-105^{\circ}$ & & & 31 & & & 0.7506 & & & \\
\hline$-90^{\circ}$ & & & & & & & & & \\
\hline & & & & & & & & & \\
\hline$-60^{\circ}$ & & & & & & & & & \\
\hline$-45^{\circ}$ & & & & & & & & & \\
\hline$-30^{\circ}$ & & & & & & & & & \\
\hline$-15^{\circ}$ & & & & & & & & & \\
\hline $0^{\circ}$ & & $1.0^{\circ}$ & 7 & 6823 & 0.6665 & 0.7204 & 12 & & \\
\hline $15^{\circ}$ & & & & & & & & & \\
\hline $30^{\circ}$ & & & & & & 0.7636 & & & \\
\hline $45^{\circ}$ & & & & & & & & & \\
\hline $60^{\circ}$ & & & & & & 0.5 & & & 0.27 \\
\hline $75^{\circ}$ & 2.425 & 4.841 & 1.78 & & & & & & \\
\hline $90^{\circ}$ & & & & & & 0.2 & & & \\
\hline & & 1.77 & & & & 0.14 & & & \\
\hline $120^{\circ}$ & & & & & & & & & 0.0336 \\
\hline & & & & 1 & & 0.0923 & 0.0 & & \\
\hline $150^{\circ}$ & 18.216 & & & & & & & & \\
\hline & & & & & & 0.29 & & & \\
\hline $180^{\circ}$ & 5 & 5 & & & & 0.4367 & 0.3126 & 0.2348 & 0.1021 \\
\hline
\end{tabular}


Table 4.6: Data for mapping the normalized plastic zone $r_{p}(\theta) / h$ for $\phi=50^{\circ}$

\begin{tabular}{|c|c|c|c|c|c|c|c|c|c|}
\hline \multirow{2}{*}{$\begin{array}{c}\text { Theta } \\
\theta\end{array}$} & \multicolumn{9}{|c|}{ Normalized T-stress, $t_{y}$} \\
\hline & -1 & -0.75 & -0.5 & -0.25 & 0 & 0.25 & 0.5 & & 1 \\
\hline$-180^{\circ}$ & 2601 & 340 & 4446 & 9 & 9275 & 5329 & .0040 & 3422 & .003 \\
\hline$-165^{\circ}$ & 3249 & 7 & & & & 706 & & & .616 \\
\hline$-150^{\circ}$ & & 0.4995 & & & 394 & 7670 & & & .383 \\
\hline-13 & 66 & 0.60 & 26 & & 1.0695 & 1.5368 & & & 45.787 \\
\hline$-120^{\circ}$ & & 0.69 & & 0 & 0.8985 & 1.1778 & & & 2.818 \\
\hline-10 & & & & & & & & & 4166 \\
\hline$-90^{\circ}$ & 273 & 0.6029 & 9 & 21 & 0.4544 & 0.5167 & 08 & & 4.1526 \\
\hline$-75^{\circ}$ & & & & & & 3457 & & & 50 \\
\hline$-60^{\circ}$ & 192 & 0.2381 & & 0.2 & 0.2524 & 0.3139 & 0.4 & & 4.5268 \\
\hline$-45^{\circ}$ & & & & & & 33 & & & 3735 \\
\hline$-30^{\circ}$ & & & & & & 04 & & & .265 \\
\hline$-15^{\circ}$ & & & & & 556 & & & & 9.5853 \\
\hline $0^{\circ}$ & & & & 24 & 9135 & 0.9799 & & & 5.1352 \\
\hline $15^{\circ}$ & & & & & & 1.0003 & & & 2.1414 \\
\hline $30^{\circ}$ & & & & & 0880 & 0.9175 & 0.8 & & 0.9652 \\
\hline $45^{\circ}$ & & & & & 1.0040 & 0.7674 & & & 0.5118 \\
\hline $60^{\circ}$ & & 6.09 & & 1.2773 & 0.8234 & 0.5873 & 0.4 & 0.3606 & 0.3018 \\
\hline $75^{\circ}$ & & 4.94 & & 0.9569 & 0.5935 & 0.4074 & 0.2992 & & 0.1843 \\
\hline $90^{\circ}$ & 29.2 & 3.28 & & 0.6 & 0.3756 & 0.2538 & 0.1 & 0.1391 & 0.1093 \\
\hline $105^{\circ}$ & & 2.02 & & & 2281 & 0.1534 & & 332 & 0.0651 \\
\hline $120^{\circ}$ & 1 & & & & & 0.1306 & & 731 & 0.0581 \\
\hline $135^{\circ}$ & & 2.15 & & & 0.2758 & 0.1933 & & & 0.0934 \\
\hline $150^{\circ}$ & & & & & 0.4609 & 0.3202 & 0.2381 & & 0.1506 \\
\hline $165^{\circ}$ & & & & & 0.6987 & 0.4736 & & & 0.2060 \\
\hline $180^{\circ}$ & 5.003 & & & & 0.9275 & 0.6209 & 0.4446 & 0.3340 & 0.2601 \\
\hline
\end{tabular}


Table 4.7: Data for mapping the normalized plastic zone $r_{p}(\theta) / h$ for $\phi=60^{\circ}$

\begin{tabular}{|c|c|c|c|c|c|c|c|c|c|}
\hline \multirow{2}{*}{$\begin{array}{c}\text { Theta } \\
\boldsymbol{\theta}\end{array}$} & \multicolumn{9}{|c|}{ Normalized T-stress, $t_{y}$} \\
\hline & -1 & -0.75 & -0.5 & -0.25 & 0 & 0.25 & 0.5 & 0.75 & 1 \\
\hline$-180^{\circ}$ & 0.3322 & 0.4266 & 0.5680 & 0.7933 & 1.1850 & 1.9588 & 3.8388 & 10.661 & 95.860 \\
\hline$-165^{\circ}$ & 0.3891 & 0.4920 & 0.6450 & 0.8873 & 1.3054 & 2.1253 & 4.1022 & 11.219 & 99.306 \\
\hline$-150^{\circ}$ & 0.4682 & 0.5614 & 0.7008 & 0.9200 & 1.2934 & 2.0121 & 3.7067 & 9.6519 & 81.016 \\
\hline$-135^{\circ}$ & 0.5812 & 0.6283 & 0.7214 & 0.8796 & 1.1534 & 1.6735 & 2.8636 & 6.8580 & 51.904 \\
\hline$-120^{\circ}$ & 0.7221 & 0.6635 & 0.6832 & 0.7630 & 0.9237 & 1.2375 & 1.9395 & 4.1658 & 26.796 \\
\hline$-105^{0}$ & 0.8055 & 0.6135 & 0.5697 & 0.5891 & 0.6663 & 0.8339 & 1.2098 & 2.3437 & 12.496 \\
\hline$-90^{\circ}$ & 0.6583 & 0.4585 & 0.4076 & 0.4085 & 0.4496 & 0.5477 & 0.7701 & 1.4284 & 6.9638 \\
\hline$-75^{\circ}$ & 0.3774 & 0.2952 & 0.2779 & 0.2902 & 0.3311 & 0.4181 & 0.6126 & 1.2031 & 6.5894 \\
\hline$-60^{\circ}$ & 0.2633 & 0.2432 & 0.2512 & 0.2813 & 0.3413 & 0.4582 & 0.7198 & 1.5509 & 10.028 \\
\hline$-45^{\circ}$ & 0.3388 & 0.3229 & 0.3401 & 0.3867 & 0.4756 & 0.6473 & 1.0326 & 2.2688 & 15.140 \\
\hline$-30^{\circ}$ & 0.6520 & 0.5559 & 0.5481 & 0.5922 & 0.6963 & 0.9058 & 1.3734 & 2.8246 & 16.860 \\
\hline$-15^{\circ}$ & 1.6106 & 1.0404 & 0.8936 & 0.8740 & 0.9420 & 1.1236 & 1.5420 & 2.7629 & 12.494 \\
\hline $0^{\circ}$ & 4.8586 & 1.9540 & 1.3853 & 1.1883 & 1.1450 & 1.2209 & 1.4695 & 2.1733 & 6.1187 \\
\hline $15^{\circ}$ & 14.422 & 3.4170 & 1.9656 & 1.4628 & 1.2489 & 1.1798 & 1.2321 & 1.4785 & 2.4522 \\
\hline $30^{\circ}$ & 31.406 & 5.1347 & 2.4624 & 1.6072 & 1.2241 & 1.0317 & 0.9449 & 0.9453 & 1.0819 \\
\hline $45^{\circ}$ & 47.031 & 6.2763 & 2.6401 & 1.5525 & 1.0761 & 0.8254 & 0.6811 & 0.5977 & 0.5583 \\
\hline $60^{\circ}$ & 50.764 & 6.1191 & 2.3741 & 1.3008 & 0.8437 & 0.6056 & 0.4656 & 0.3768 & 0.3180 \\
\hline $75^{\circ}$ & 41.813 & 4.8106 & 1.7899 & 0.9431 & 0.5890 & 0.4070 & 0.3009 & 0.2336 & 0.1881 \\
\hline $90^{\circ}$ & 28.688 & 3.2481 & 1.1903 & 0.6180 & 0.3804 & 0.2591 & 0.1887 & 0.1443 & 0.1144 \\
\hline $105^{\circ}$ & 19.973 & 2.2830 & 0.8443 & 0.4422 & 0.2746 & 0.1887 & 0.1387 & 0.1070 & 0.0856 \\
\hline $120^{\circ}$ & 19.224 & 2.2781 & 0.8708 & 0.4706 & 0.3013 & 0.2134 & 0.1618 & 0.1290 & 0.1071 \\
\hline $135^{\circ}$ & 27.873 & 3.3419 & 1.2906 & 0.7042 & 0.4549 & 0.3252 & 0.2490 & 0.2006 & 0.1683 \\
\hline $150^{\circ}$ & 47.250 & 5.5034 & 2.0711 & 1.1032 & 0.6963 & 0.4863 & 0.3635 & 0.2853 & 0.2327 \\
\hline $165^{\circ}$ & 73.903 & 8.3293 & 3.0388 & 1.5709 & 0.9627 & 0.6529 & 0.4736 & 0.3604 & 0.2844 \\
\hline $180^{\circ}$ & 95.860 & 10.661 & 3.8388 & 1.9588 & 1.1850 & 0.7933 & 0.5680 & 0.4266 & 0.3322 \\
\hline
\end{tabular}


Table 4.8: Data for mapping the normalized plastic zone $r_{p}(\theta) / h$ for $\phi=70^{\circ}$

\begin{tabular}{|c|c|c|c|c|c|c|c|c|c|}
\hline \multirow{2}{*}{$\begin{array}{c}\text { Theta } \\
\boldsymbol{\theta}\end{array}$} & \multicolumn{9}{|c|}{ Normalized T-stress, $t_{y}$} \\
\hline & -1 & -0.75 & -0.5 & -0.25 & 0 & 0.25 & 0.5 & 0.75 & 1 \\
\hline$-180^{\circ}$ & 0.3908 & 0.5021 & 0.6685 & 0.9338 & 1.3951 & 2.3065 & 4.5211 & 12.558 & 112.93 \\
\hline$-165^{\circ}$ & 0.4335 & 0.5485 & 0.7194 & 0.9901 & 1.4575 & 2.3742 & 4.5852 & 12.546 & 111.12 \\
\hline$-150^{\circ}$ & 0.4913 & 0.5913 & 0.7405 & 0.9752 & 1.3750 & 2.1454 & 3.9645 & 10.358 & 87.269 \\
\hline$-135^{\circ}$ & 0.5630 & 0.6182 & 0.7181 & 0.8843 & 1.1703 & 1.7138 & 2.9618 & 7.1766 & 55.163 \\
\hline$-120^{\circ}$ & 0.6207 & 0.5987 & 0.6354 & 0.7266 & 0.8984 & 1.2294 & 1.9727 & 4.3669 & 29.487 \\
\hline$-105^{\circ}$ & 0.5841 & 0.5007 & 0.4952 & 0.5363 & 0.6317 & 0.8235 & 1.2514 & 2.5815 & 15.493 \\
\hline$-90^{\circ}$ & 0.4127 & 0.3519 & 0.3469 & 0.3748 & 0.4407 & 0.5733 & 0.8692 & 1.7877 & 10.670 \\
\hline$-75^{\circ}$ & 0.2672 & 0.2547 & 0.2683 & 0.3050 & 0.3751 & 0.5105 & 0.8144 & 1.7893 & 11.940 \\
\hline$-60^{\circ}$ & 0.2640 & 0.2708 & 0.2994 & 0.3538 & 0.4508 & 0.6356 & 1.0540 & 2.4309 & 17.458 \\
\hline$-45^{\circ}$ & 0.4109 & 0.4088 & 0.4427 & 0.5145 & 0.6455 & 0.8962 & 1.4614 & 3.3012 & 22.993 \\
\hline$-30^{\circ}$ & 0.8254 & 0.7117 & 0.7061 & 0.7665 & 0.9049 & 1.1822 & 1.8009 & 3.7271 & 22.496 \\
\hline$-15^{\circ}$ & 2.0236 & 1.2929 & 1.1051 & 1.0771 & 1.1576 & 1.3766 & 1.8827 & 3.3570 & 15.015 \\
\hline $0^{\circ}$ & 5.9040 & 2.3211 & 1.6313 & 1.3911 & 1.3339 & 1.4152 & 1.6935 & 2.4829 & 6.8334 \\
\hline $15^{0}$ & 16.447 & 3.8425 & 2.1970 & 1.6281 & 1.3850 & 1.3038 & 1.3558 & 1.6171 & 2.6448 \\
\hline $30^{\circ}$ & 33.291 & 5.4405 & 2.6084 & 1.7022 & 1.2962 & 1.0923 & 1.0002 & 1.0004 & 1.1445 \\
\hline $45^{\circ}$ & 46.660 & 6.2727 & 2.6527 & 1.5670 & 1.0907 & 0.8401 & 0.6963 & 0.6143 & 0.5781 \\
\hline $60^{\circ}$ & 47.680 & 5.8129 & 2.2772 & 1.2587 & 0.8233 & 0.5959 & 0.4622 & 0.3777 & 0.3224 \\
\hline $75^{\circ}$ & 38.024 & 4.4418 & 1.6761 & 0.8950 & 0.5662 & 0.3964 & 0.2971 & 0.2338 & 0.1912 \\
\hline $90^{\circ}$ & 26.680 & 3.0992 & 1.1635 & 0.6183 & 0.3893 & 0.2713 & 0.2023 & 0.1584 & 0.1288 \\
\hline $105^{\circ}$ & 20.971 & 2.5125 & 0.9696 & 0.5287 & 0.3414 & 0.2439 & 0.1866 & 0.1502 & 0.1260 \\
\hline $120^{\circ}$ & 23.855 & 2.9609 & 1.1774 & 0.6596 & 0.4370 & 0.3203 & 0.2518 & 0.2089 & 0.1815 \\
\hline $135^{\circ}$ & 37.327 & 4.5675 & 1.7949 & 0.9949 & 0.6525 & 0.4735 & 0.3683 & 0.3019 & 0.2586 \\
\hline $150^{\circ}$ & 62.205 & 7.2794 & 2.7513 & 1.4714 & 0.9323 & 0.6537 & 0.4906 & 0.3868 & 0.3169 \\
\hline $165^{\circ}$ & 92.267 & 10.402 & 3.7963 & 1.9631 & 1.2035 & 0.8164 & 0.5924 & 0.4510 & 0.3559 \\
\hline $180^{\circ}$ & 112.93 & 12.558 & 4.5211 & 2.3065 & 1.3951 & 0.9338 & 0.6685 & 0.5021 & 0.3908 \\
\hline
\end{tabular}


Table 4.9: Data for mapping the normalized plastic zone $r_{p}(\theta) / h$ for $\phi=80^{\circ}$

\begin{tabular}{|c|c|c|c|c|c|c|c|c|c|}
\hline \multirow{2}{*}{$\begin{array}{l}\text { Theta } \\
\theta\end{array}$} & \multicolumn{9}{|c|}{ Normalized T-stress, $t_{y}$} \\
\hline & -1 & -0.75 & -0.5 & -0.25 & 0 & 0.25 & 0.5 & 0.75 & 1 \\
\hline$-180^{\circ}$ & 0.4295 & 517 & 1345 & 0259 & 1.5326 & 5335 & 4.9655 & 3.791 & 4.01 \\
\hline$-165^{\circ}$ & & & & & 1.5266 & .4875 & & & \\
\hline & 4859 & 5867 & 73 & 9730 & 1.3755 & 1517 & & 46 & 297 \\
\hline & 156 & & 6744 & 0.8385 & 1.1195 & 1.6540 & 2.8856 & & \\
\hline$-120^{\circ}$ & 5073 & 5116 & 91 & 0.6545 & 0.8267 & 1.1555 & & & \\
\hline$-105^{\circ}$ & 141 & & & 0.4 & 5744 & 7802 & 1.2 & & \\
\hline$-90^{\circ}$ & 2788 & 2752 & 0.29 & 0.34 & 0.4287 & 0.5929 & 0.9 & & \\
\hline$-75^{\circ}$ & & & 0. & 0.3 & & 148 & & & \\
\hline$-60^{\circ}$ & & 7 & 23 & 0.43 & 0.5670 & 0.8252 & 1.4167 & & \\
\hline$-45^{\circ}$ & & & & & & & & & \\
\hline & & & 0. & & 1.0882 & 1.4250 & & & \\
\hline$-15^{\circ}$ & & 4970 & 1.27 & 1.2 & 1.3258 & 1.5721 & 2.1 & & \\
\hline $0^{\circ}$ & 4 & 3 & 6 & 1. & 4574 & 1.5395 & 1.83 & & 1914 \\
\hline $15^{\circ}$ & & 4.0619 & 2.31 & & 1.4469 & 1.3579 & 1.40 & & 2.65 \\
\hline $30^{\circ}$ & & & & & & 1.0931 & & & \\
\hline $45^{\circ}$ & & 9 & & 1.4 & 1.0470 & 0.8107 & $0.6^{\circ}$ & & 0.5706 \\
\hline $60^{\circ}$ & & 5.2070 & 2.0 & & 0.7 & 0.5604 & 0.4 & & 0.31 \\
\hline $75^{\circ}$ & & & & & & 0.3777 & 0.2 & & \\
\hline $90^{\circ}$ & & 2.8515 & & & & 0.2899 & & & \\
\hline & & & & & & 134 & & & \\
\hline $120^{\circ}$ & 544 & 3.5763 & 1.47 & 0.8517 & 0.5809 & 0.4384 & 0.3555 & & 0.2778 \\
\hline & & & & & 0.8437 & 0.6197 & 0.4882 & 0.4060 & \\
\hline $150^{\circ}$ & 74.936 & 8.8052 & 3.3403 & 1.7 & & 0.8018 & 0.6038 & 0.47 & 0.3930 \\
\hline $165^{\circ}$ & 106.46 & 12.009 & 4.38 & 2.26 & 1.3913 & 0.9443 & 0.6855 & 221 & 0.4123 \\
\hline $180^{\circ}$ & 124.01 & 13.791 & 4.9655 & 2.5335 & 1.5326 & 1.0259 & 0.7345 & 0.5517 & 0.4295 \\
\hline
\end{tabular}


Table 4.10: Data for mapping the normalized plastic zone $r_{p}(\theta) / h$ for $\phi=90^{\circ}$

\begin{tabular}{|c|c|c|c|c|c|c|c|c|c|}
\hline \multirow{2}{*}{$\begin{array}{c}\text { Theta } \\
\theta\end{array}$} & \multicolumn{9}{|c|}{ Normalized T-stress, $t_{y}$} \\
\hline & -1 & -0.75 & -0.5 & -0.25 & 0 & 0.25 & 0.5 & 0.75 & 1 \\
\hline$-180^{\circ}$ & 0.4429 & 0.5688 & 0.7573 & 1.0577 & 1.5800 & 2.6117 & 5.1186 & 14.216 & 127.82 \\
\hline$-165^{\circ}$ & 0.4462 & 0.5648 & 0.7412 & 1.0207 & 1.5033 & 2.4500 & 4.7340 & 12.960 & 114.85 \\
\hline$-150^{\circ}$ & 0.4517 & 0.5472 & 0.6893 & 0.9126 & 1.2934 & 2.0285 & 3.7687 & 9.9030 & 83.980 \\
\hline$-135^{\circ}$ & 0.4429 & 0.5005 & 0.5945 & 0.7464 & 1.0058 & 1.4997 & 2.6423 & 6.5470 & 51.787 \\
\hline$-120^{\circ}$ & 0.3895 & 0.4102 & 0.4620 & 0.5544 & 0.7163 & 1.0240 & 1.7242 & 4.0508 & 29.864 \\
\hline$-105^{\circ}$ & 0.2848 & 0.2952 & 0.3288 & 0.3909 & 0.5007 & 0.7098 & 1.1841 & 2.7508 & 19.962 \\
\hline$-90^{\circ}$ & 0.2016 & 0.2206 & 0.2557 & 0.3142 & 0.4150 & 0.6066 & 1.0460 & 2.5284 & 19.371 \\
\hline$-75^{\circ}$ & 0.2039 & 0.2330 & 0.2793 & 0.3535 & 0.4800 & 0.7212 & 1.2808 & 3.2027 & 25.623 \\
\hline$-60^{\circ}$ & 0.3011 & 0.3390 & 0.4016 & 0.5030 & 0.6763 & 1.0061 & 1.7683 & 4.3695 & 34.443 \\
\hline$-45^{\circ}$ & 0.5365 & 0.5574 & 0.6217 & 0.7401 & 0.9492 & 1.3471 & 2.2503 & 5.2360 & 38.082 \\
\hline$-30^{\circ}$ & 1.0899 & 0.9493 & 0.9473 & 1.0329 & 1.2241 & 1.6053 & 2.4563 & 5.1128 & 31.176 \\
\hline$-15^{\circ}$ & 2.6020 & 1.6275 & 1.3781 & 1.3344 & 1.4260 & 1.6863 & 2.2912 & 4.0472 & 17.721 \\
\hline $0^{\circ}$ & 7.1429 & 2.6997 & 1.8692 & 1.5779 & 1.5000 & 1.5779 & 1.8692 & 2.6997 & 7.1429 \\
\hline $15^{\circ}$ & 17.721 & 4.0472 & 2.2912 & 1.6863 & 1.4260 & 1.3344 & 1.3781 & 1.6275 & 2.6020 \\
\hline $30^{\circ}$ & 31.176 & 5.1128 & 2.4563 & 1.6053 & 1.2241 & 1.0329 & 0.9473 & 0.9493 & 1.0899 \\
\hline $45^{\circ}$ & 38.082 & 5.2360 & 2.2503 & 1.3471 & 0.9492 & 0.7401 & 0.6217 & 0.5574 & 0.5365 \\
\hline $60^{\circ}$ & 34.443 & 4.3695 & 1.7683 & 1.0061 & 0.6763 & 0.5030 & 0.4016 & 0.3390 & 0.3011 \\
\hline $75^{\circ}$ & 25.623 & 3.2027 & 1.2808 & 0.7212 & 0.4800 & 0.3535 & 0.2793 & 0.2330 & 0.2039 \\
\hline $90^{\circ}$ & 19.372 & 2.5284 & 1.0460 & 0.6066 & 0.4150 & 0.3142 & 0.2557 & 0.2206 & 0.2016 \\
\hline $105^{\circ}$ & 19.962 & 2.7508 & 1.1841 & 0.7098 & 0.5007 & 0.3909 & 0.3288 & 0.2952 & 0.2848 \\
\hline $120^{\circ}$ & 29.864 & 4.0508 & 1.7242 & 1.0240 & 0.7173 & 0.5544 & 0.4620 & 0.4102 & 0.3895 \\
\hline $135^{\circ}$ & 51.787 & 6.5470 & 2.6423 & 1.4997 & 1.0058 & 0.7464 & 0.5945 & 0.5005 & 0.4429 \\
\hline $150^{\circ}$ & 83.980 & 9.9030 & 3.7687 & 2.0285 & 1.2934 & 0.9126 & 0.6893 & 0.5472 & 0.4517 \\
\hline $165^{\circ}$ & 114.85 & 12.960 & 4.7340 & 2.4500 & 1.5033 & 1.0207 & 0.7412 & 0.5648 & 0.4462 \\
\hline $180^{\circ}$ & 127.82 & 14.216 & 5.1186 & 2.6117 & 1.5800 & 1.0577 & 0.7573 & 0.5688 & 0.4429 \\
\hline
\end{tabular}


Table 4.11: Table of maximum plastic zone size for varying mode II to mode I SIF ratios and normalized T-stress values

\begin{tabular}{|c|c|c|c|c|c|c|c|c|c|}
\hline \multirow{2}{*}{$\phi$} & \multicolumn{9}{|c|}{ T-stress Normalized, $t_{y}$} \\
\hline & -1 & -0.75 & -0.5 & -0.25 & 0 & 0.25 & 0.5 & 0.75 & 1 \\
\hline $0^{\circ}$ & 20.675 & 2.5900 & 1.0442 & 0.5986 & 0.4161 & 0.3491 & 0.4003 & 0.7442 & 4.8724 \\
\hline $10^{\circ}$ & 28.589 & 3.4119 & 1.3123 & 0.7134 & 0.4607 & 0.5053 & 0.7569 & 1.746 & 13.685 \\
\hline $20^{\circ}$ & 36.344 & 4.2712 & 1.6262 & 0.8808 & 0.5809 & 0.7600 & 1.2863 & 3.2303 & 26.974 \\
\hline $30^{\circ}$ & 43.117 & 5.0802 & 1.9523 & 1.0792 & 0.7507 & 1.0827 & 1.9485 & 5.1032 & 43.949 \\
\hline $40^{\circ}$ & 52.841 & 5.8753 & 2.2517 & 1.2821 & 0.9438 & 1.4404 & 2.6854 & 7.2055 & 63.184 \\
\hline $50^{\circ}$ & 75.003 & 8.3422 & 3.0040 & 1.5329 & 1.1398 & 1.8005 & 3.4294 & 9.3377 & 82.751 \\
\hline $60^{\circ}$ & 95.860 & 10.661 & 3.8388 & 1.9588 & 1.3171 & 2.1265 & 4.1061 & 11.288 & 100.74 \\
\hline $70^{\circ}$ & 112.93 & 12.558 & 4.5211 & 2.3065 & 1.4579 & 2.3866 & 4.6490 & 12.857 & 115.26 \\
\hline $80^{\circ}$ & 124.01 & 13.791 & 4.9655 & 2.5335 & 1.5490 & 2.5544 & 4.9988 & 13.870 & 124.62 \\
\hline $90^{\circ}$ & 127.83 & 14.216 & 5.1186 & 2.6117 & 1.5800 & 2.6117 & 5.1186 & 14.216 & 127.83 \\
\hline
\end{tabular}

Note: $\mathrm{K}_{\mathrm{II}} / \mathrm{K}_{\mathrm{I}}=\tan \phi$ 


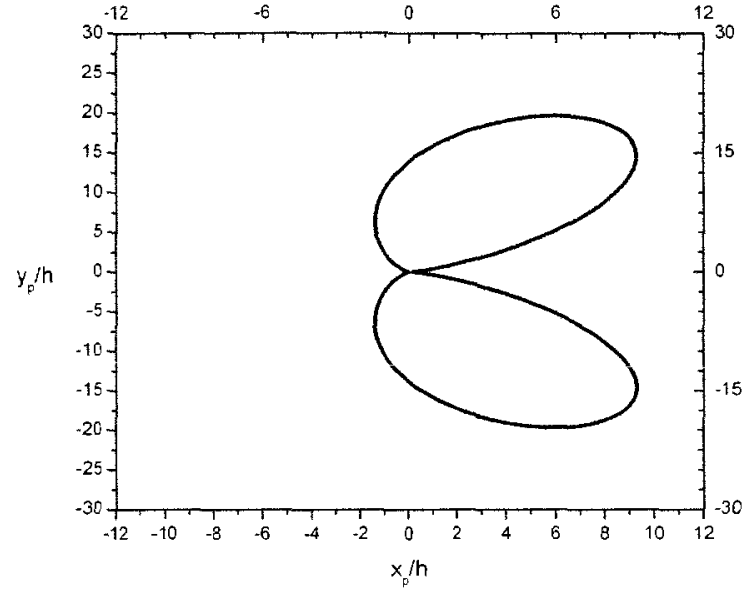

(a) $t_{y}=-1$

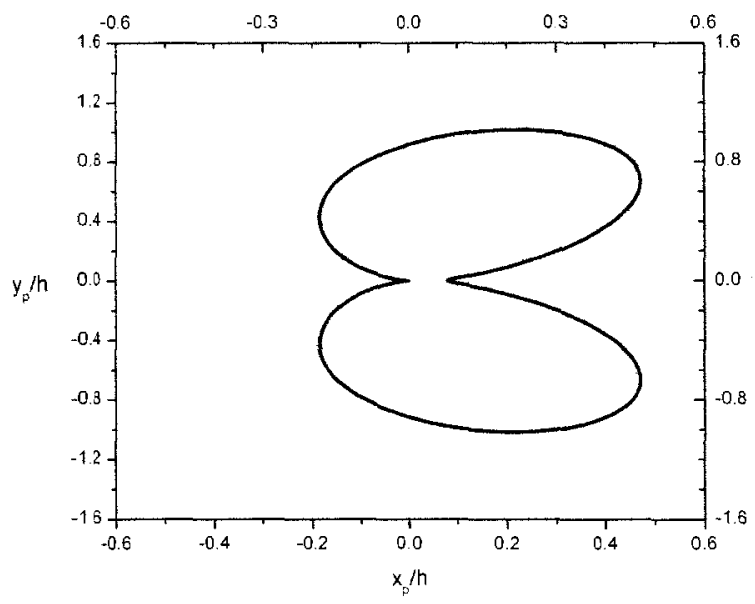

(c) $t_{y}=-0.5$

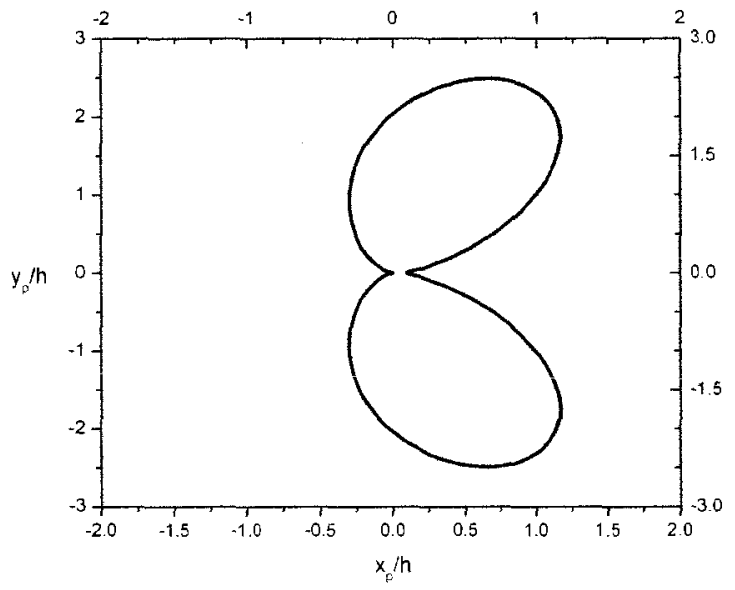

(b) $t_{y}=-0.75$

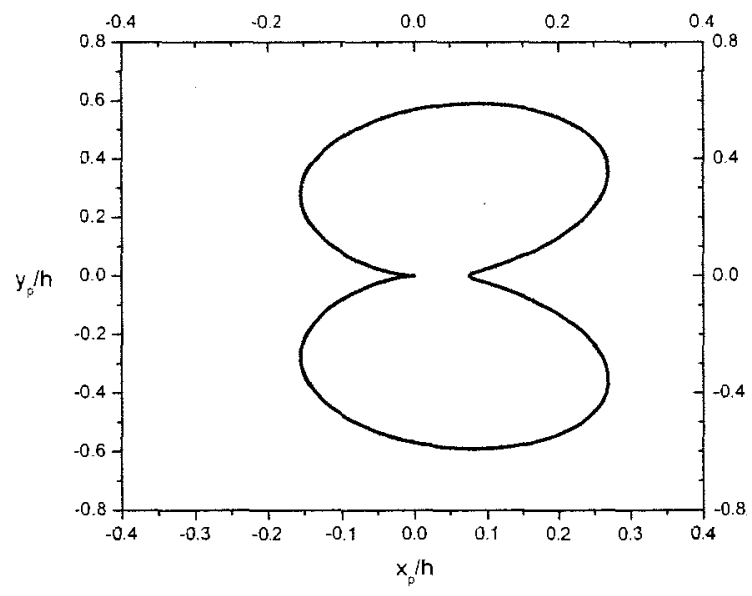

(d) $t_{y}=-0.25$

Figure 4.1: Plastic zone maps for $\phi=\theta^{\circ}$ (a)-(d)

ฉ̊ 


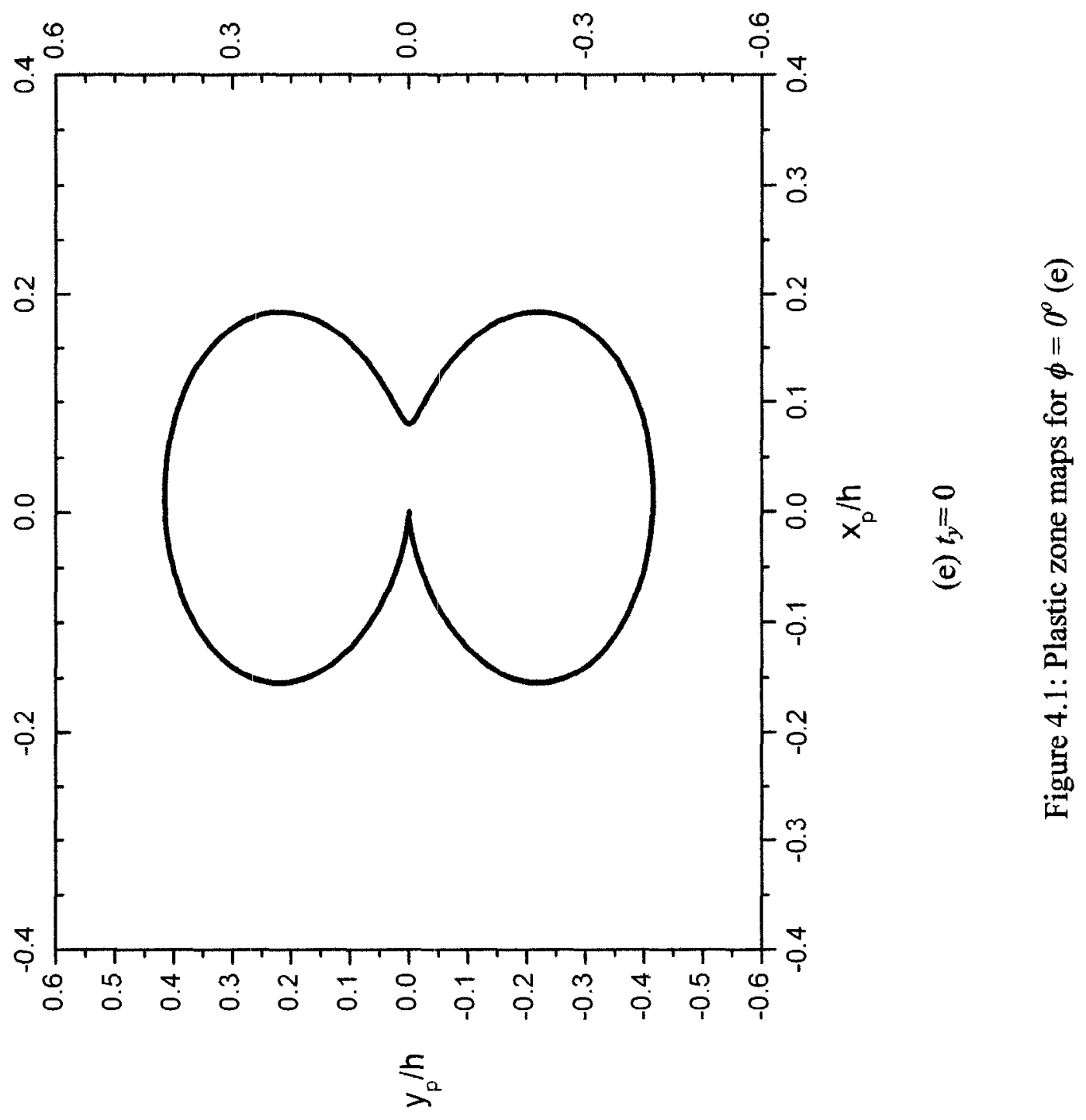




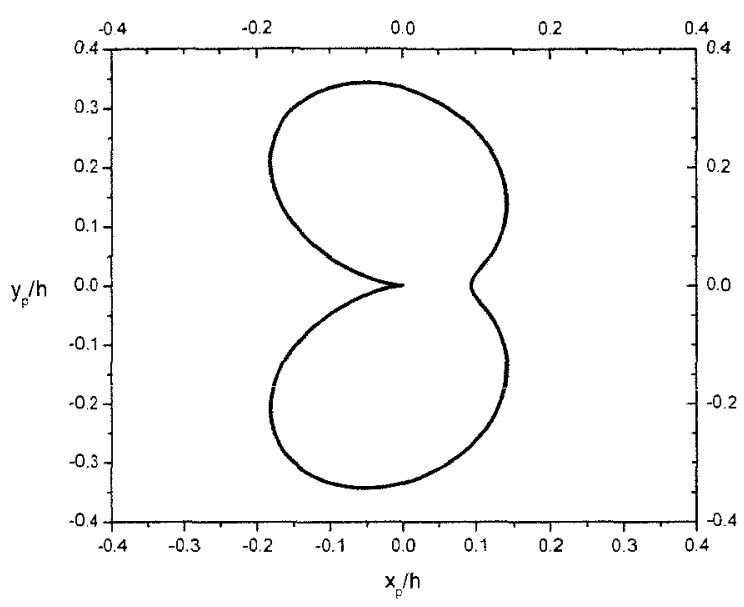

(f) $t_{y}=0.25$

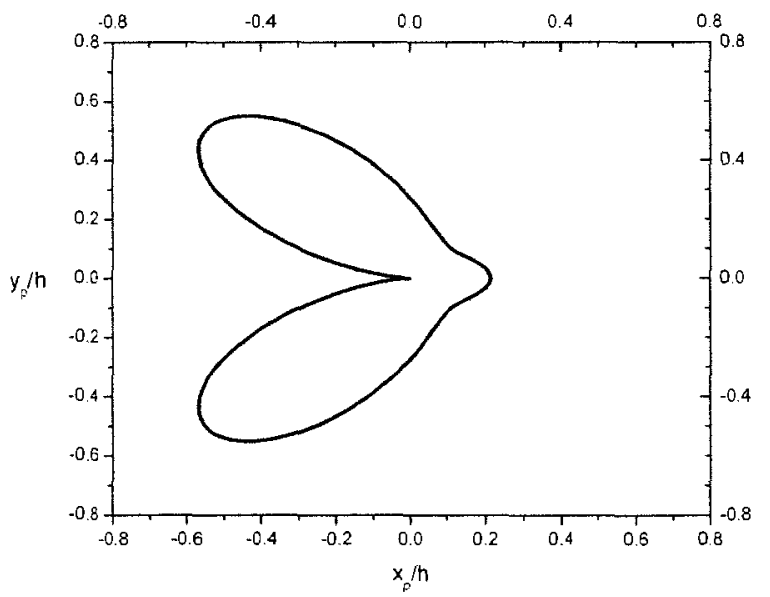

(h) $t_{y}=0.75$

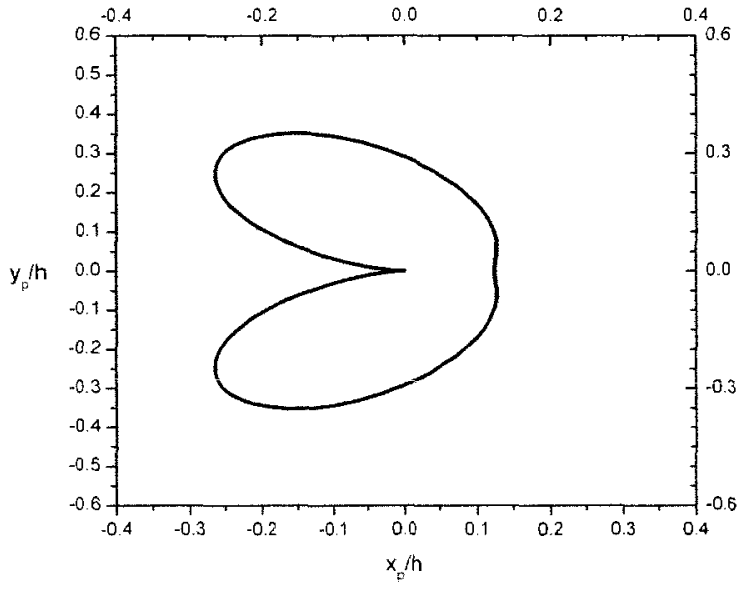

(g) $t_{y}=0.5$

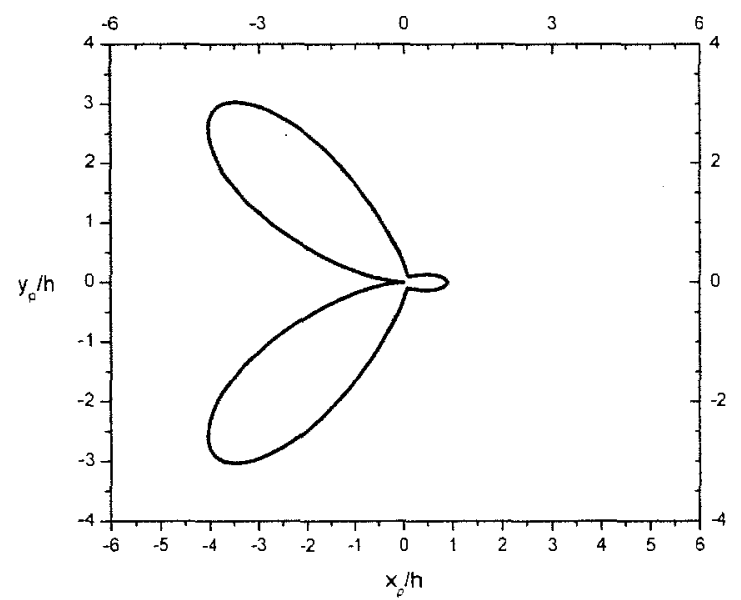

(i) $t_{y}=1$

Figure 4.1: Plastic zone maps for $\phi=0^{\circ}$ (f)-(i) 


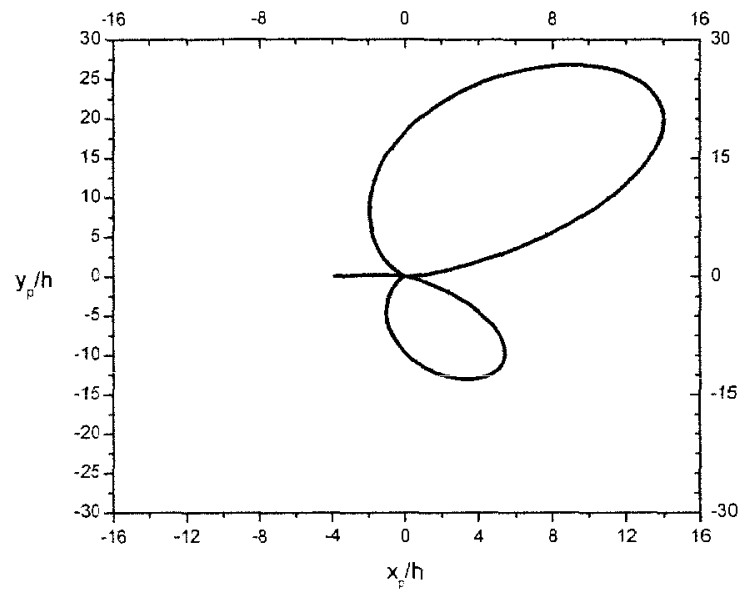

(a) $t_{y}=-1$

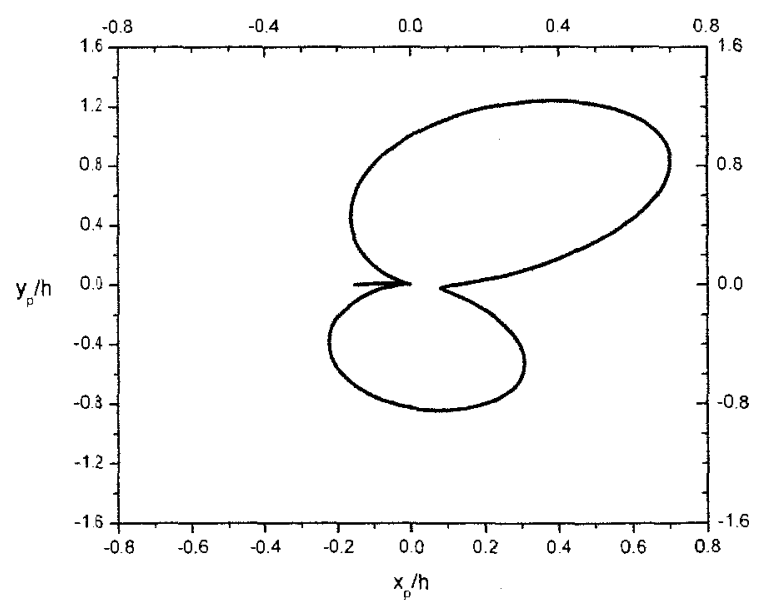

(c) $t_{y}=-0.5$

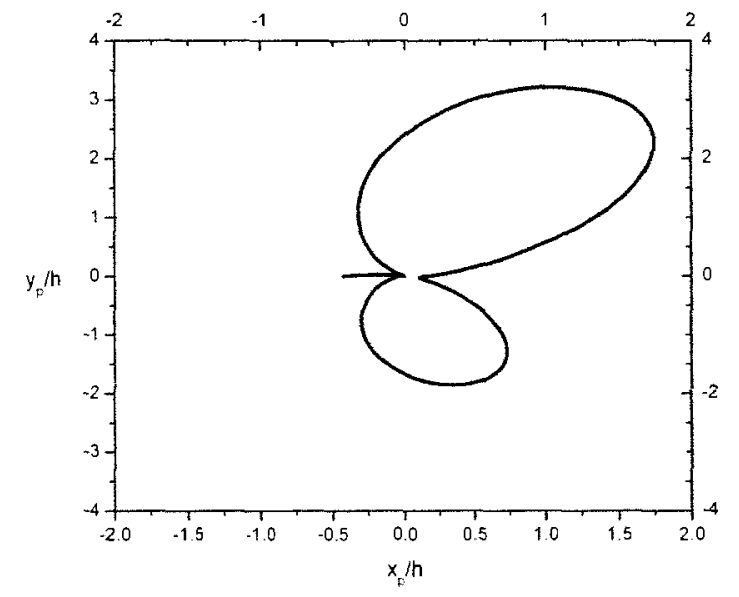

(b) $t_{y}=-0.75$

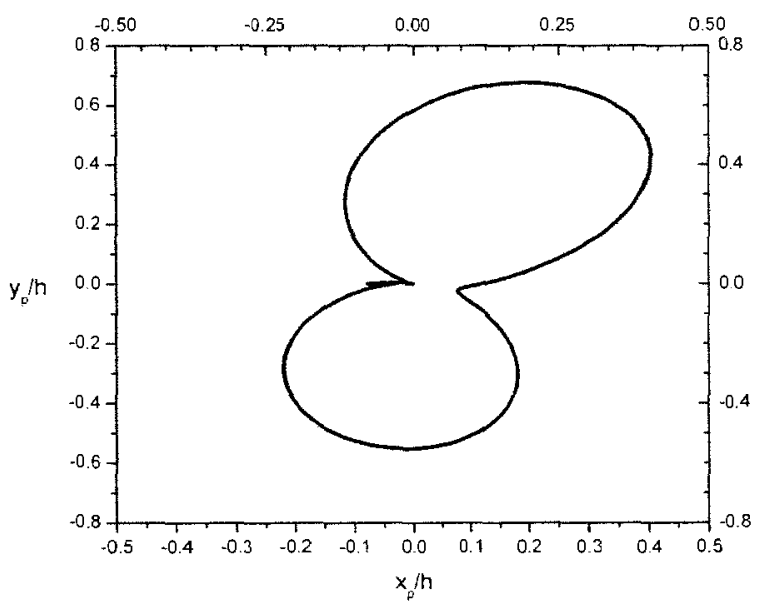

(d) $t_{y}=-0.25$

Figure 4.2: Plastic zone maps for $\phi=10^{\circ}$ (a)-(d) 


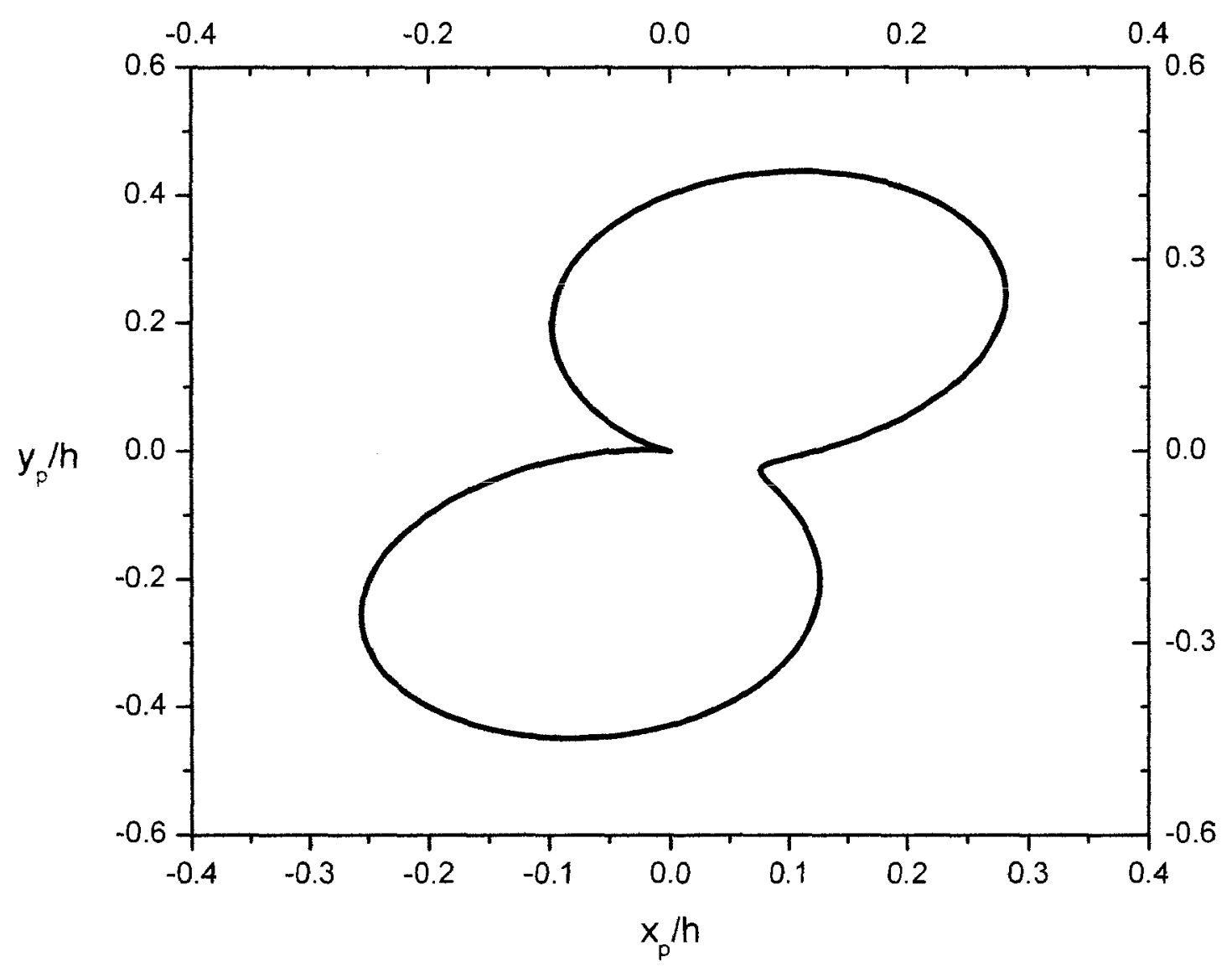

(e) $t_{y}=0$

Figure 4.2: Plastic zone maps for $\phi=10^{\circ}(\mathrm{e})$ 


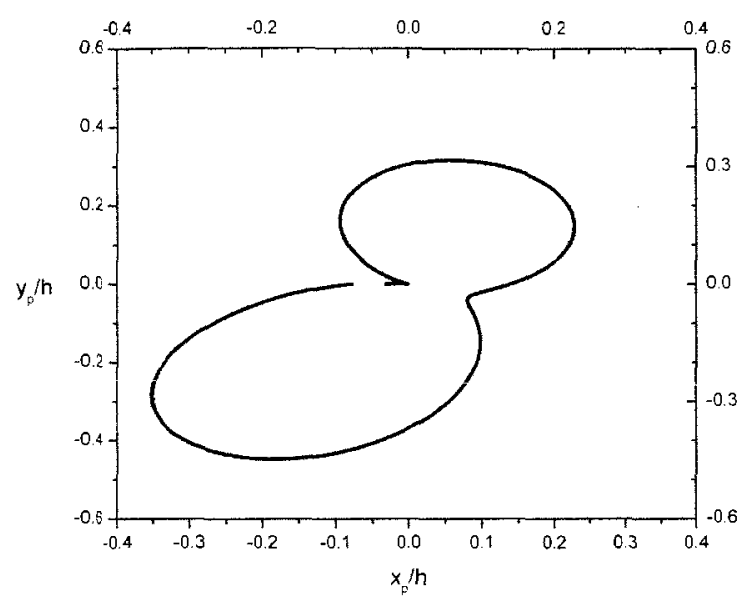

(f) $t_{y}=0.25$

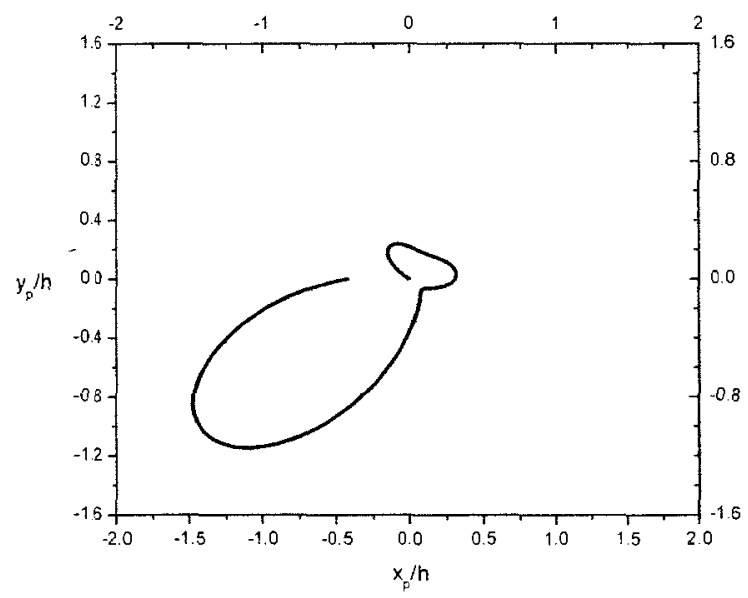

(h) $t_{y}=0.75$

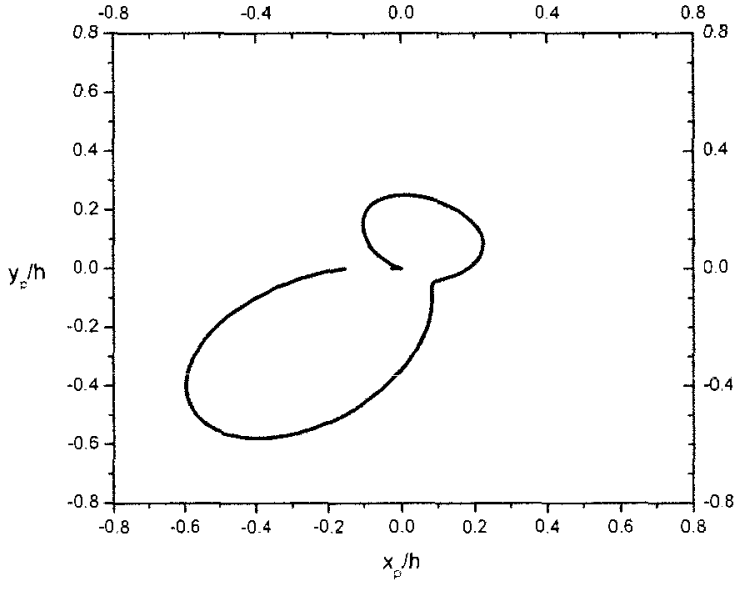

(g) $t_{y}=0.5$

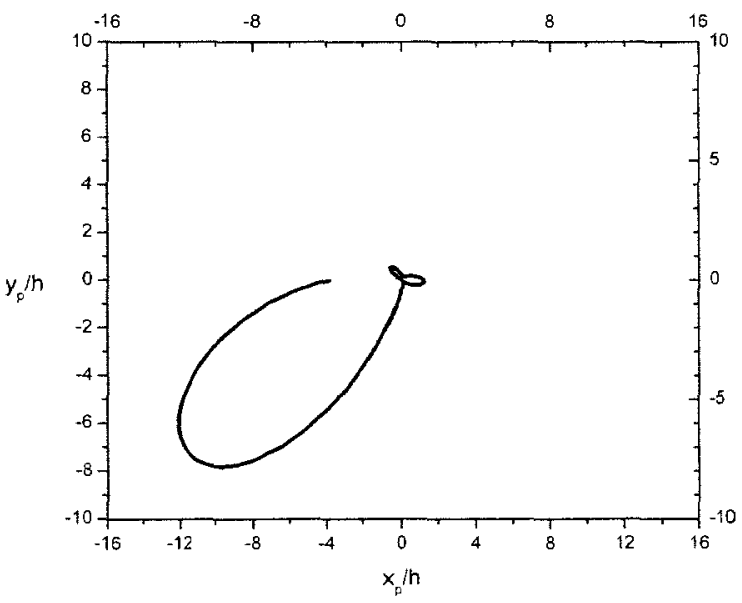

(i) $t_{y}=1$

Figure 4.2: Plastic zone maps for $\phi=10^{\circ}$ (f)-(i)

$\overrightarrow{0}$ 


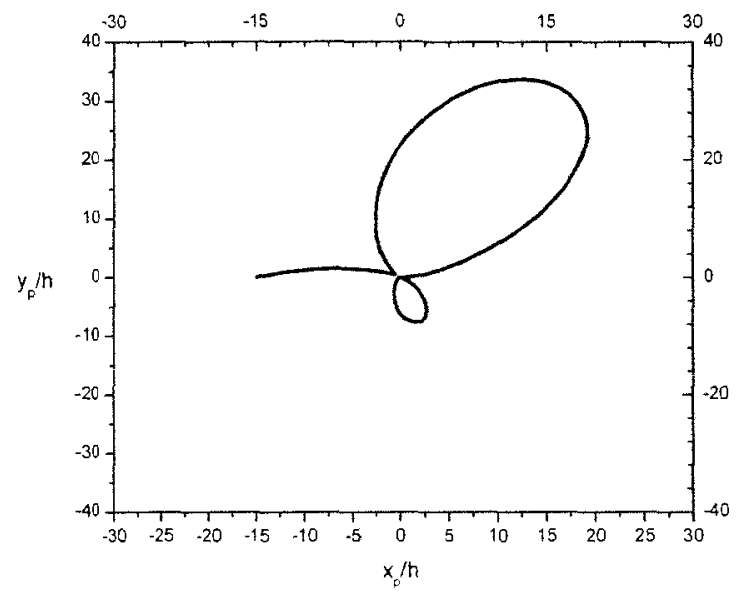

(a) $t_{y}=-1$

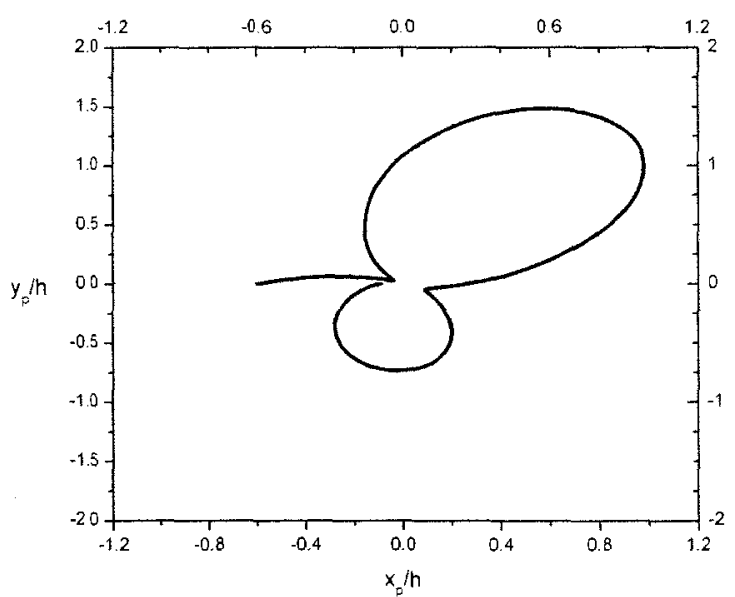

(c) $t_{y}=-0.5$

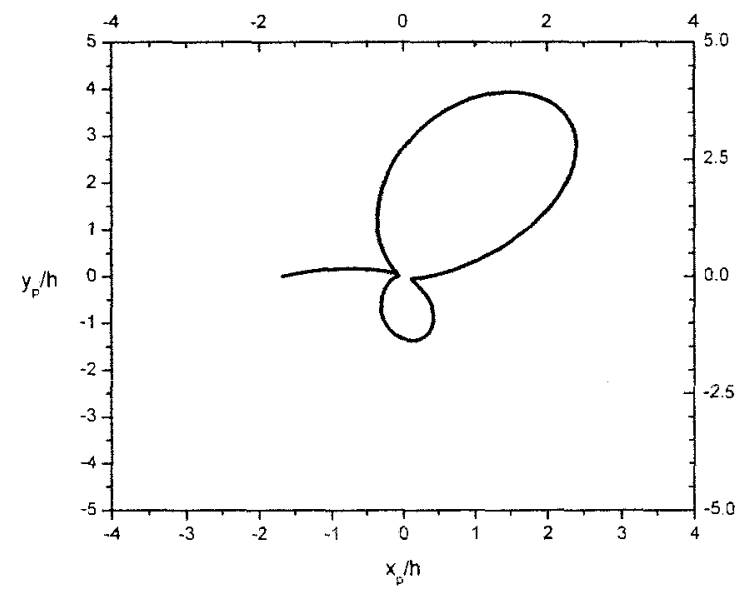

(b) $t_{y}=-0.75$

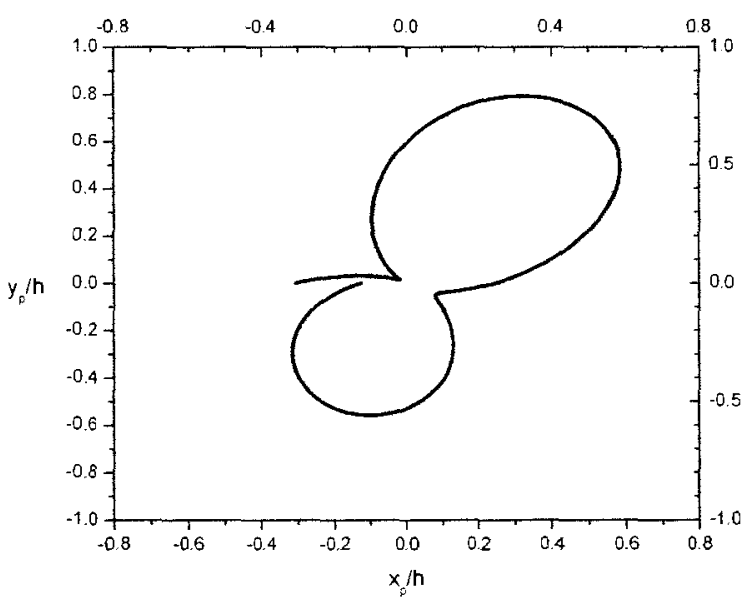

(d) $t_{y}=-0.25$

Figure 4.3: Plastic zone maps for $\phi=20^{\circ}$ (a)-(d) 


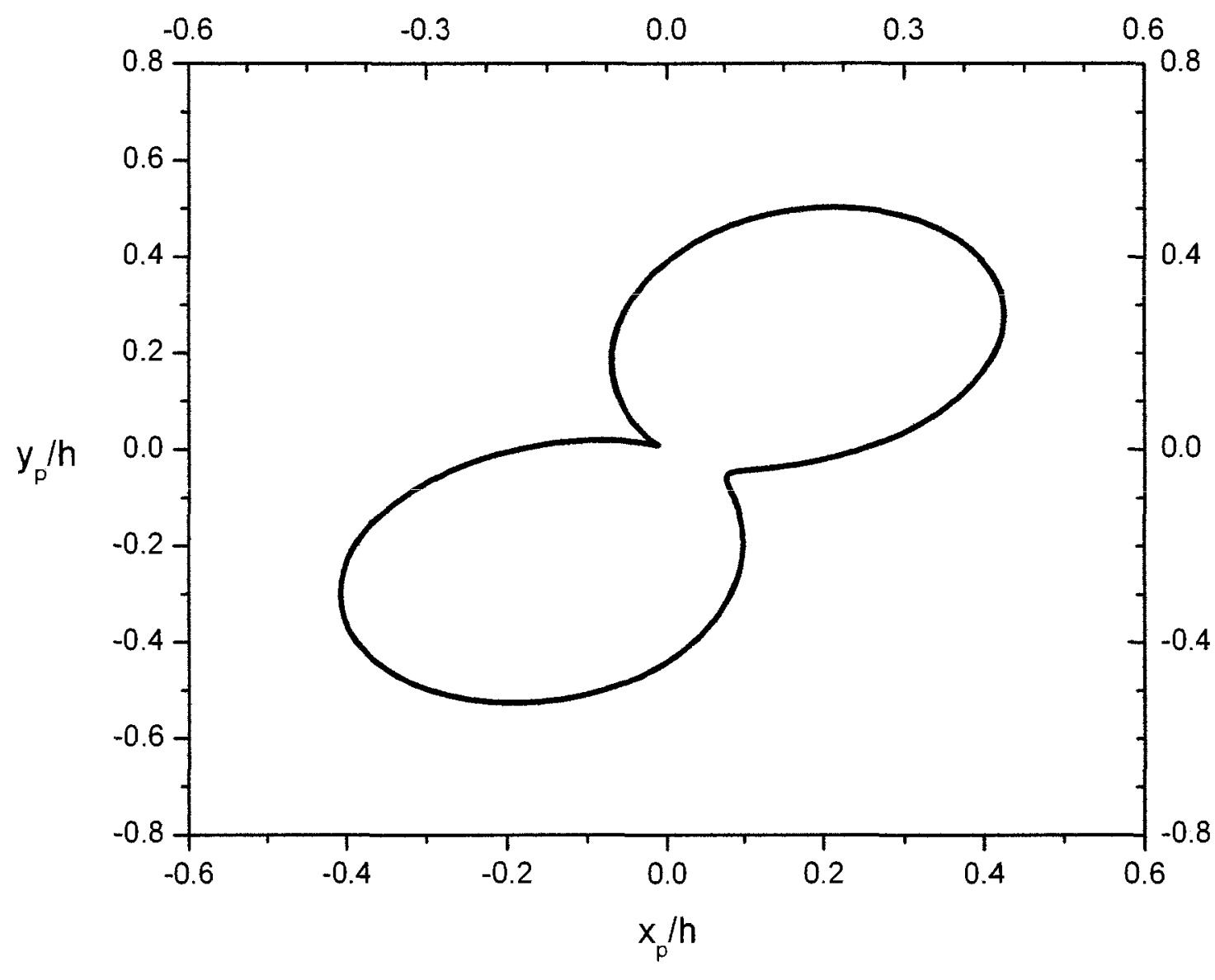

(e) $t_{y}=0$

Figure 4.3: Plastic zone maps for $\phi=20^{\circ}$ (e) 


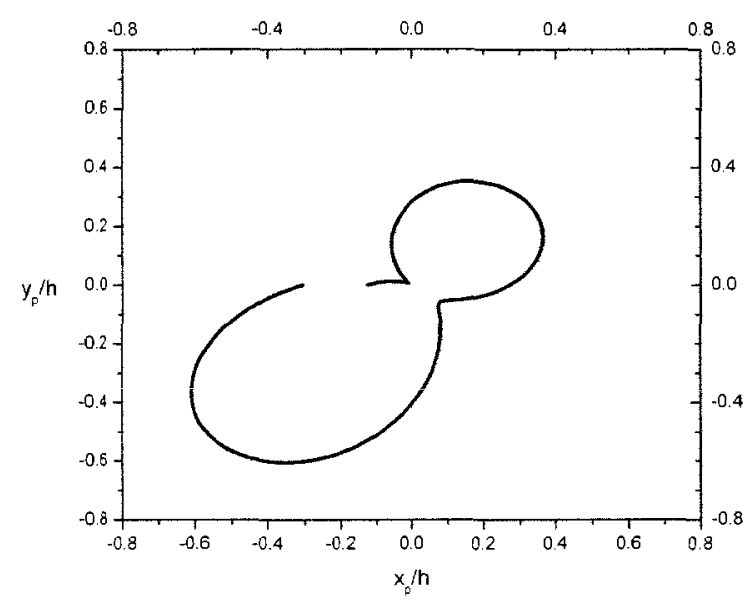

(f) $t_{y}=0.25$

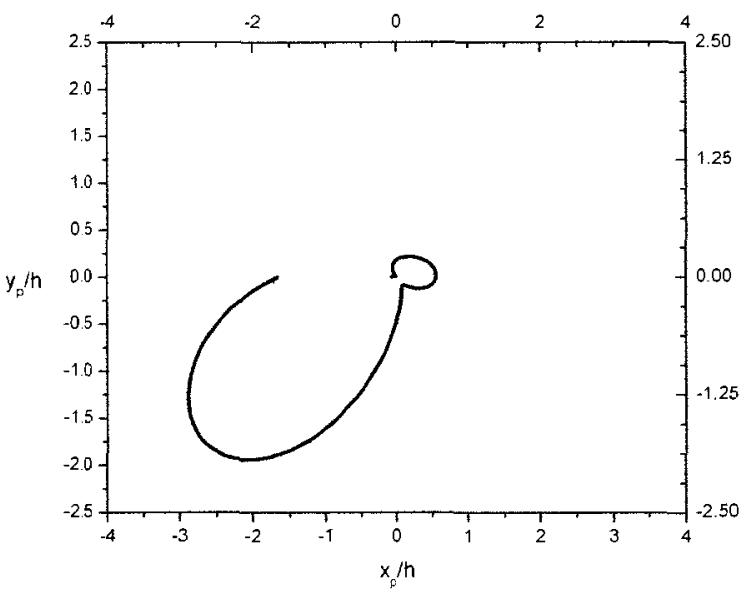

(h) $t_{y}=0.75$

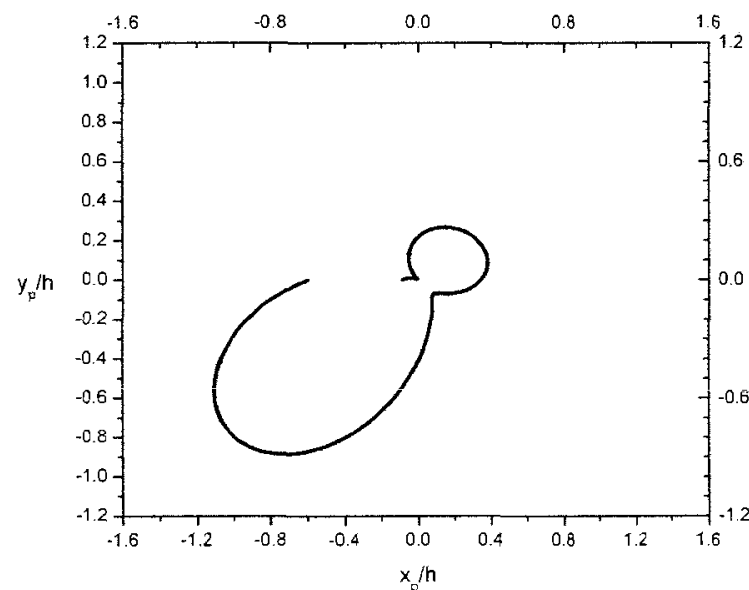

(g) $t_{y}=0.5$

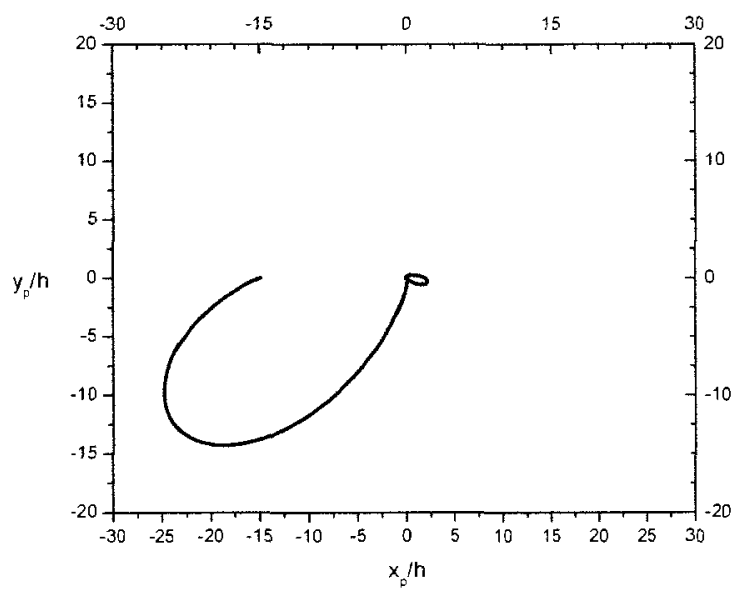

(i) $t_{y}=1$

Figure 4.3: Plastic zone maps for $\phi=20^{\circ}$ (f)-(i)

$\overrightarrow{8}$ 


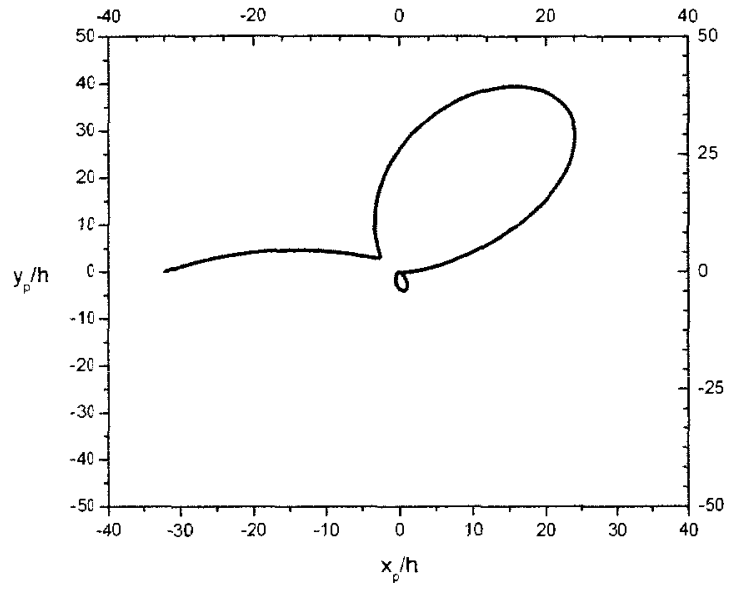

(a) $t_{y}=-1$

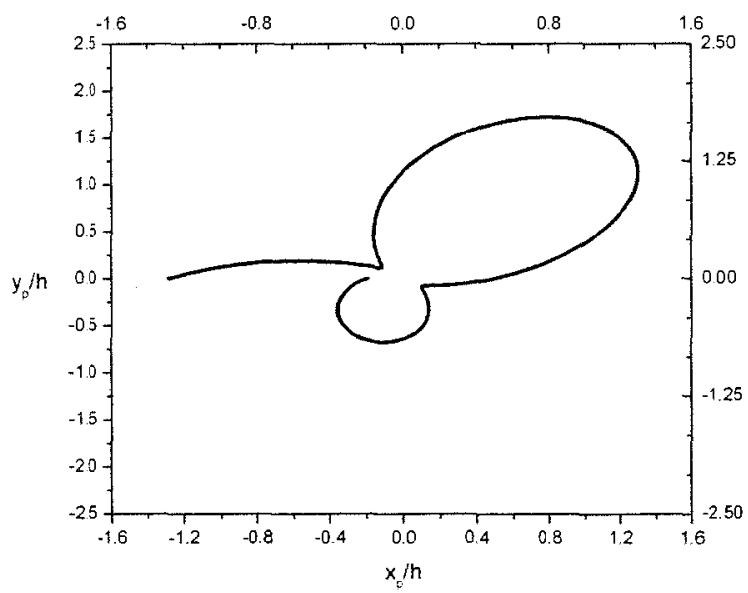

(c) $t_{y}=-0.5$

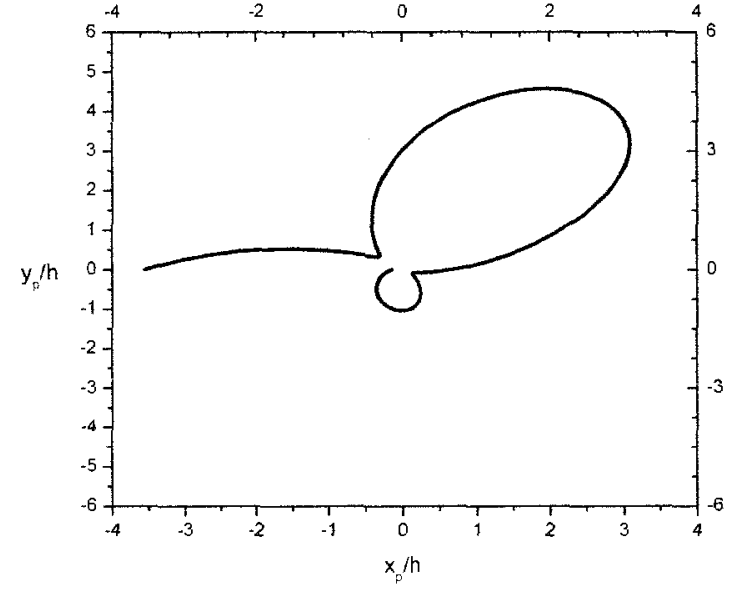

(b) $t_{y}=-0.75$

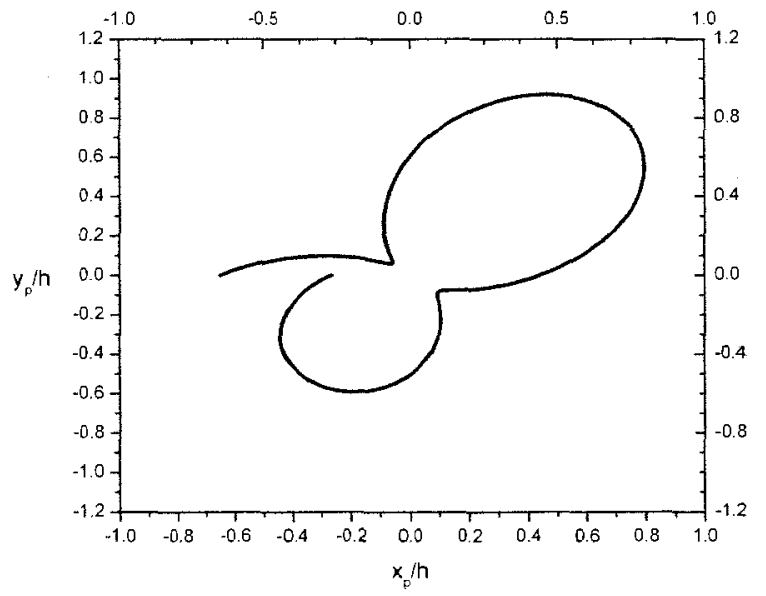

(d) $t_{y}=-0.25$

Figure 4.4: Plastic zone maps for $\phi=30^{\circ}$ (a)-(d) 


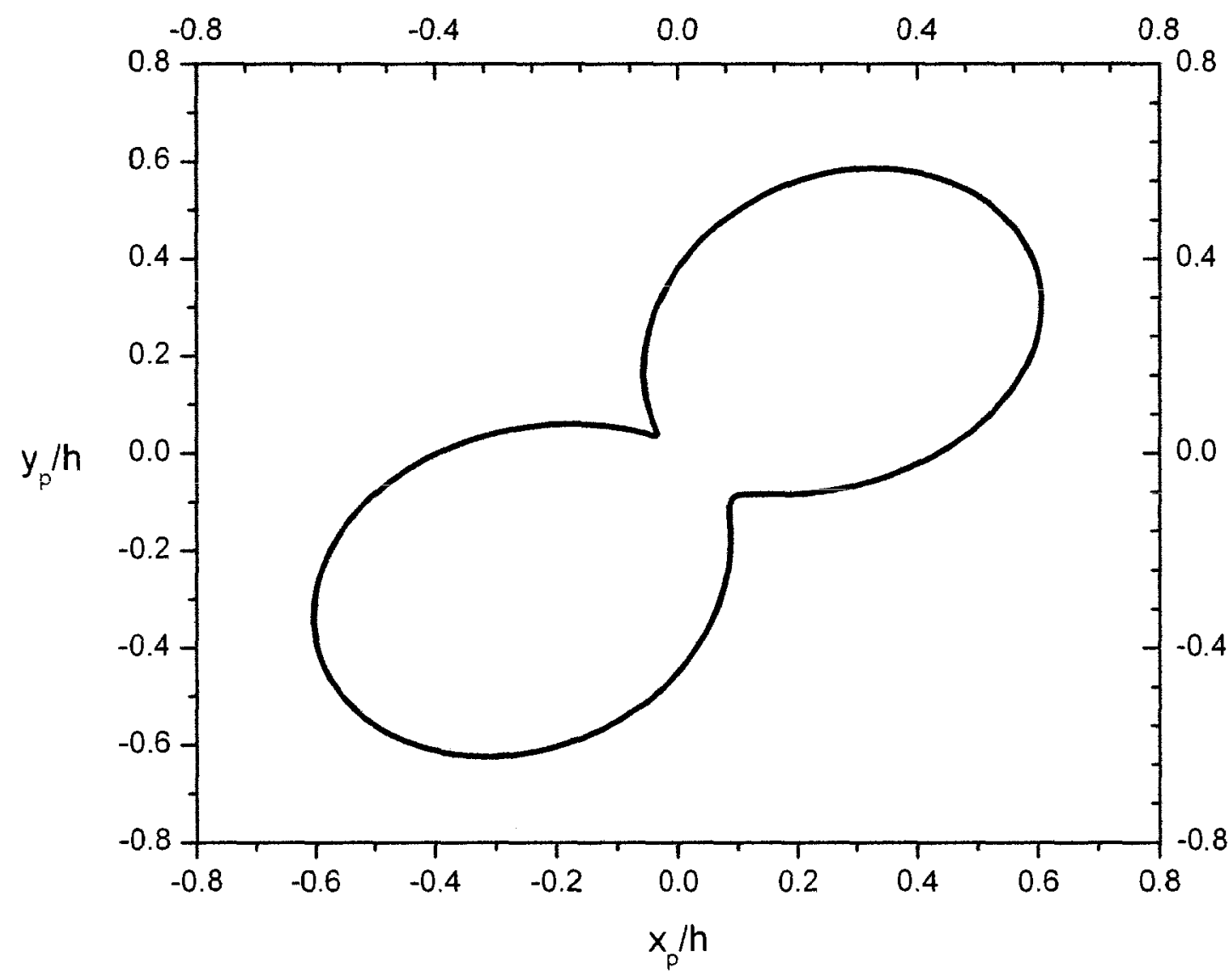

(e) $t_{y}=0$

Figure 4.4: Plastic zone maps for $\phi=30^{\circ}$ (e) 


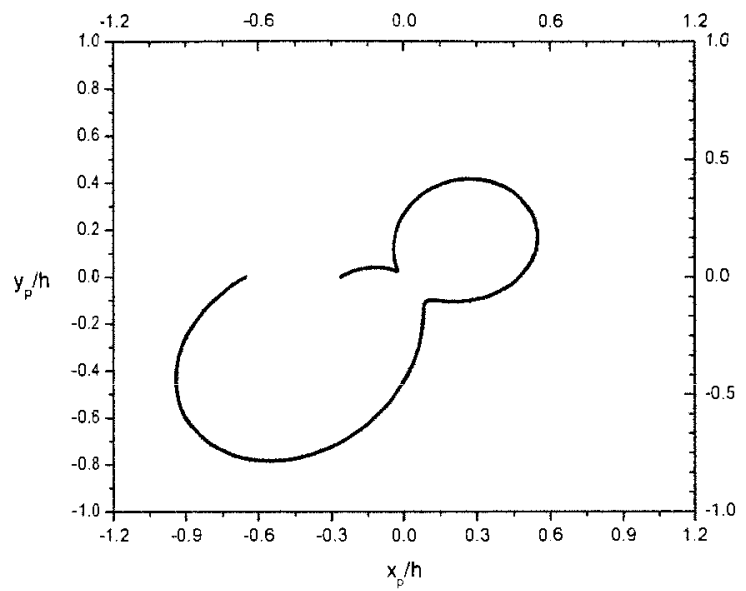

(f) $t_{y}=0.25$

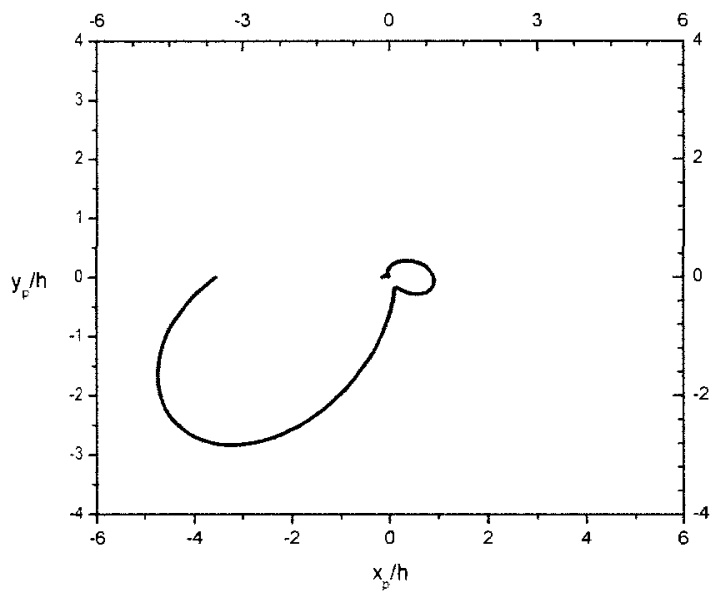

(h) $t_{y}=0.75$

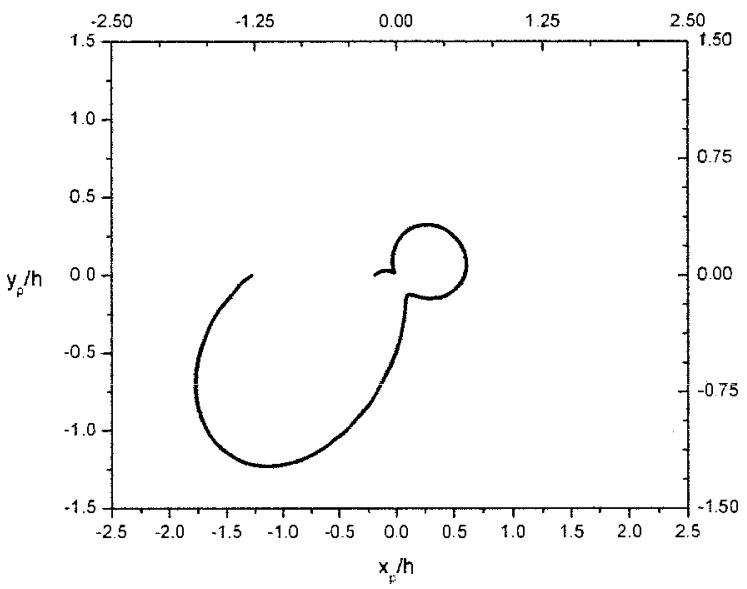

(g) $t_{y}=0.5$

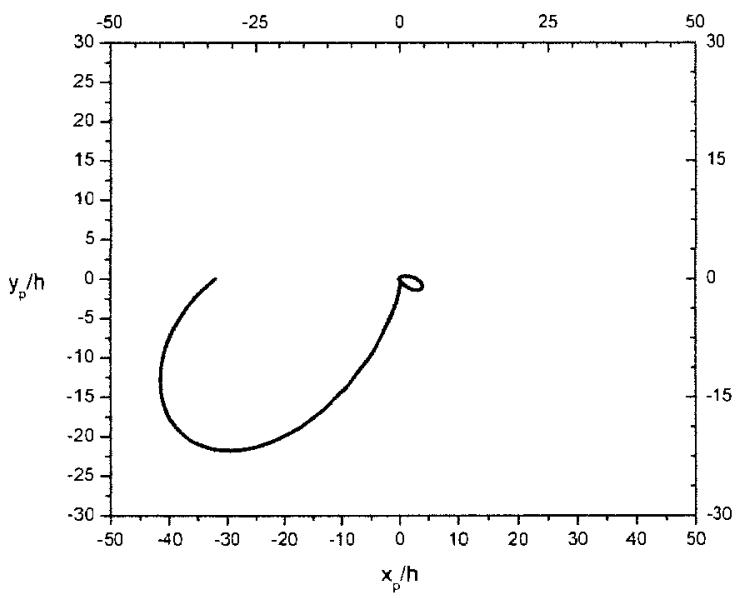

(i) $t_{y}=1$

Figure 4.4: Plastic zone maps for $\phi=30^{\circ}$ (f)-(i)

 


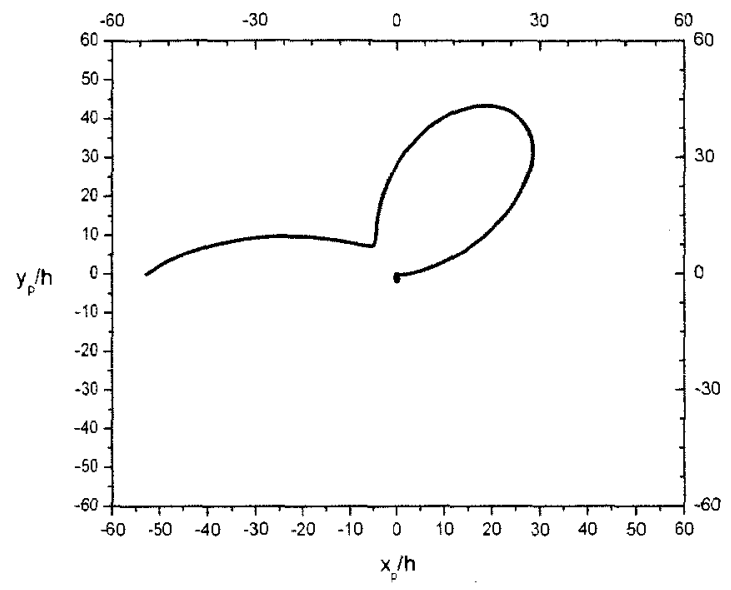

(a) $t_{y}=-1$

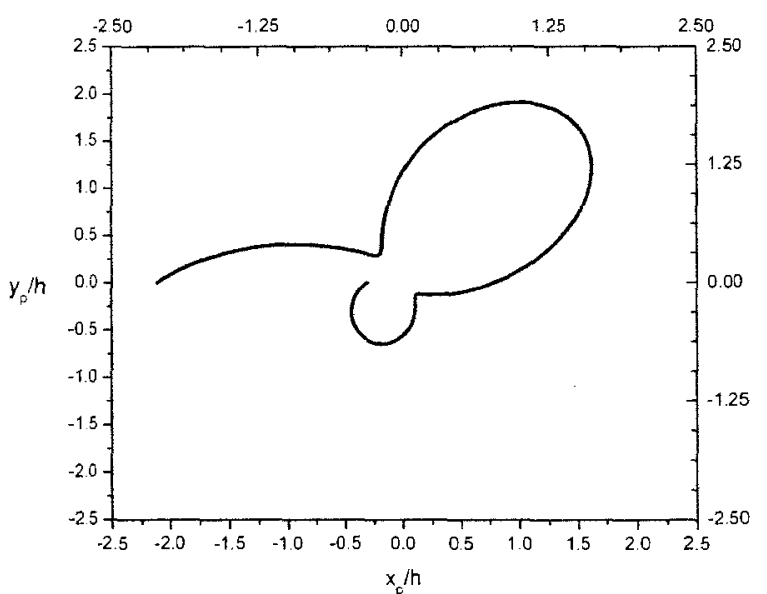

(c) $t_{y}=-0.5$

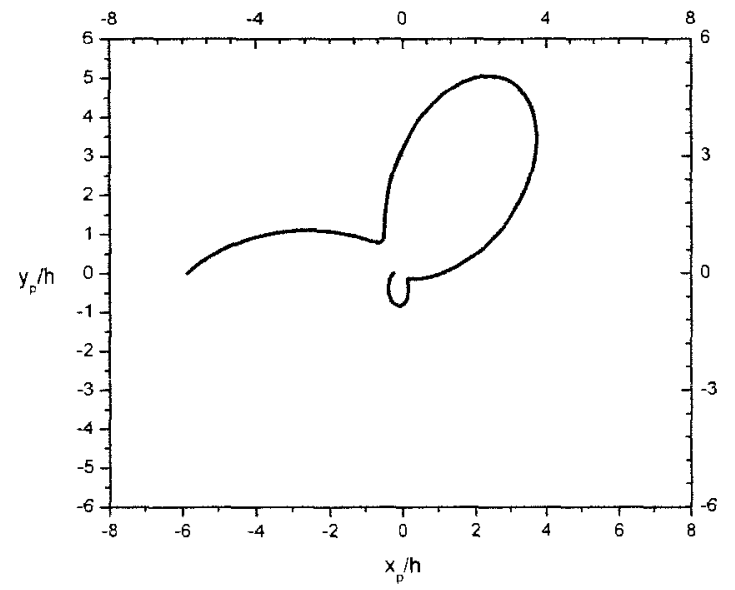

(b) $t_{y}=-0.75$

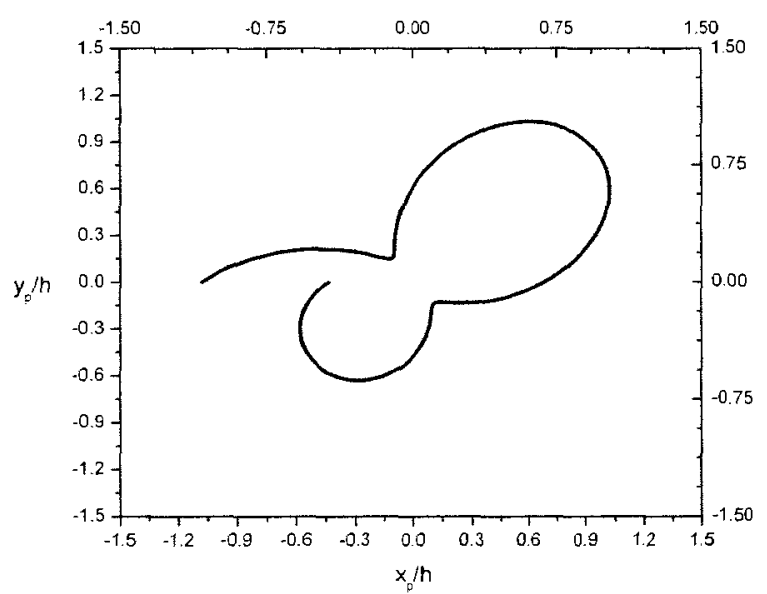

(d) $t_{y}=-0.25$

Figure 4.5: Plastic zone maps for $\phi=40^{\circ}$ (a)-(d) 


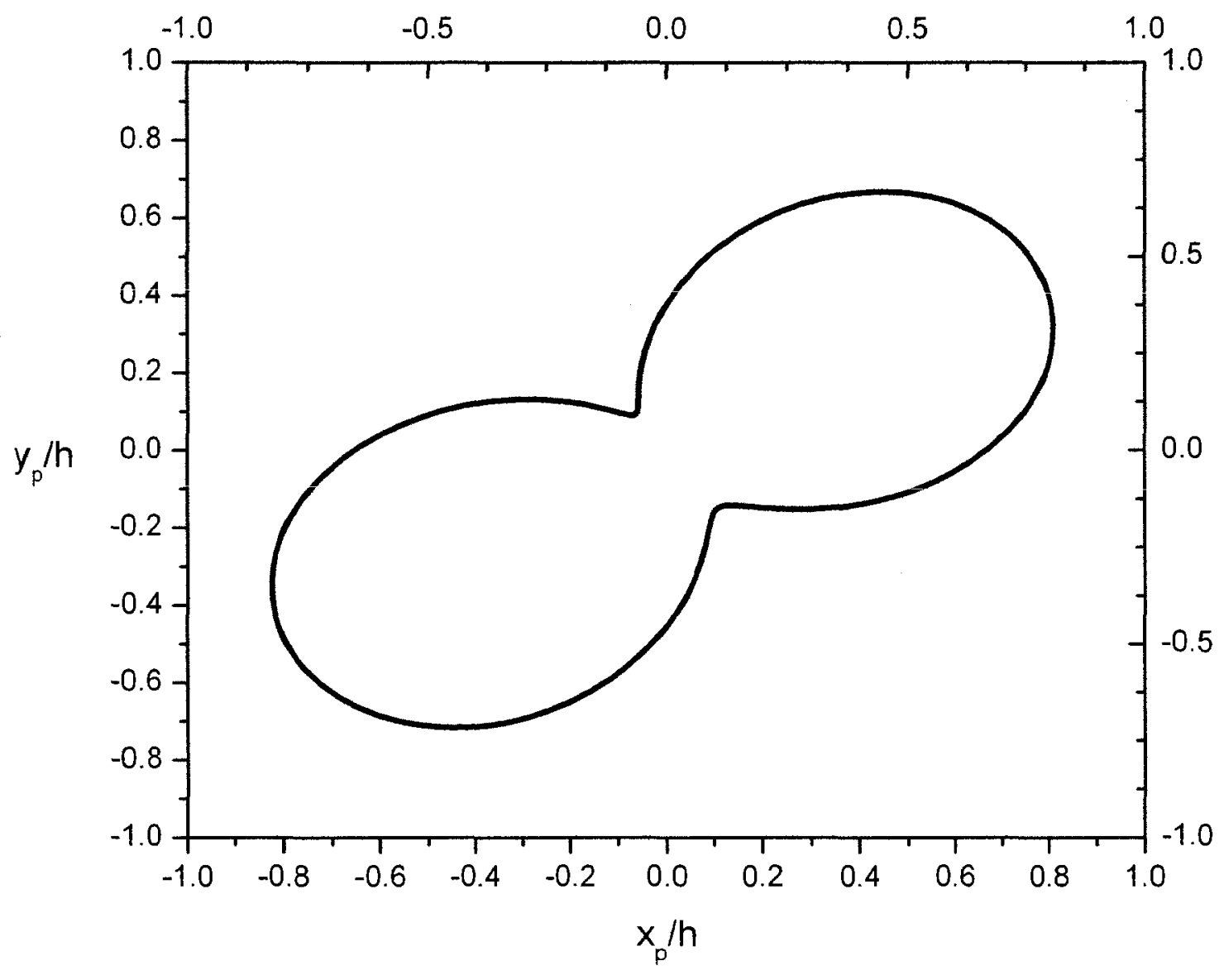

(e) $t_{y}=0$

Figure 4.5: Plastic zone maps for $\phi=40^{\circ}$ (e) 


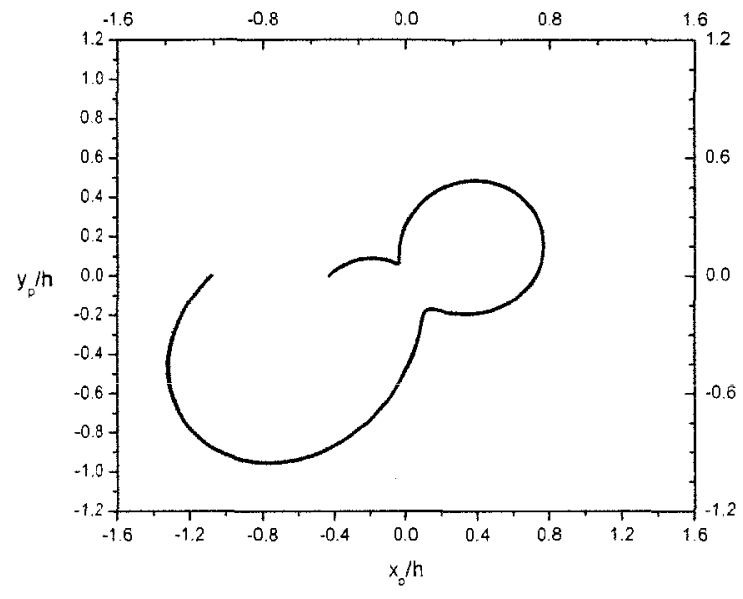

(f) $t_{y}=0.25$

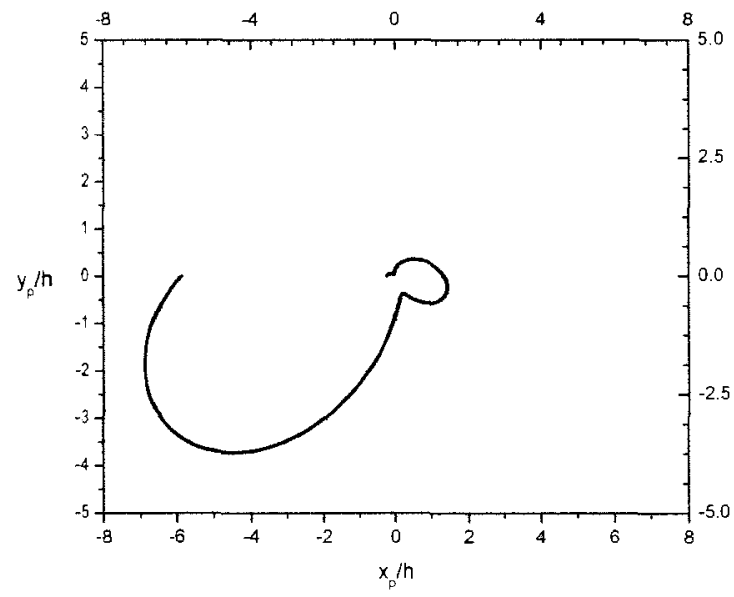

(h) $t_{y}=0.75$

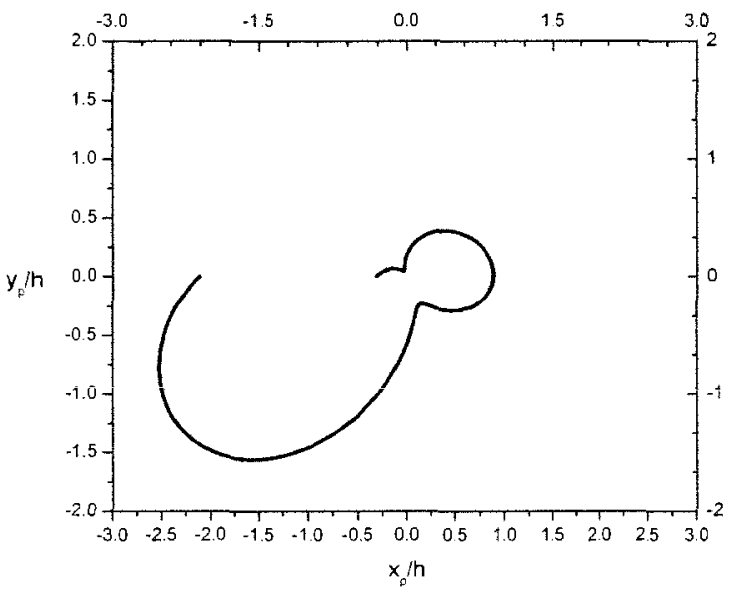

(g) $t_{y}=0.5$

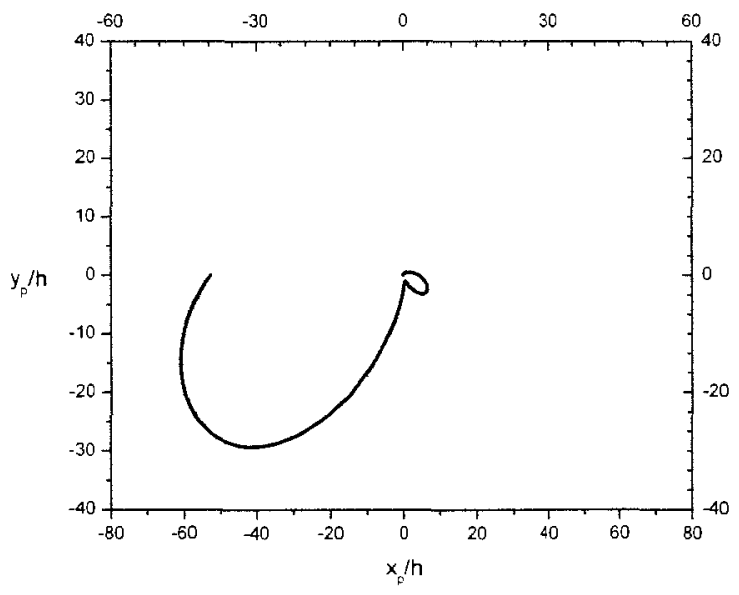

(i) $t_{y}=1$

Figure 4.5: Plastic zone maps for $\phi=40^{\circ}$ (f)-(i)

$\overline{0}$ 


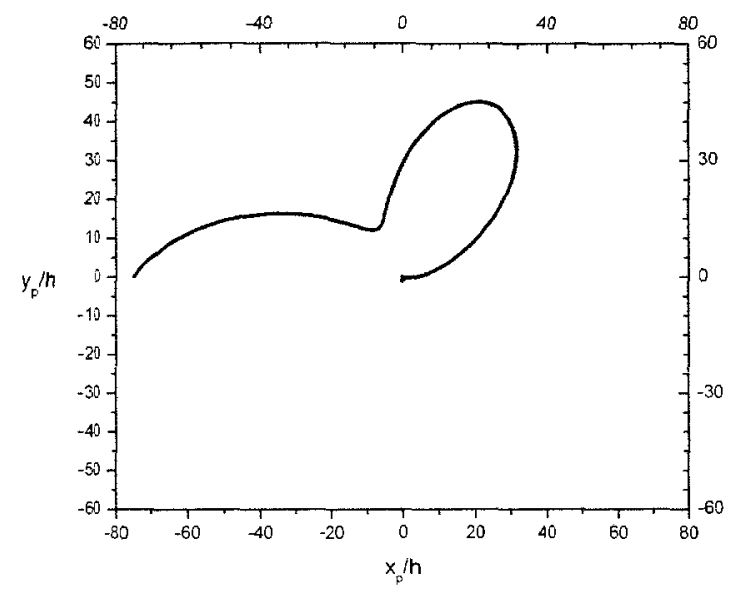

(a) $t_{y}=-1$

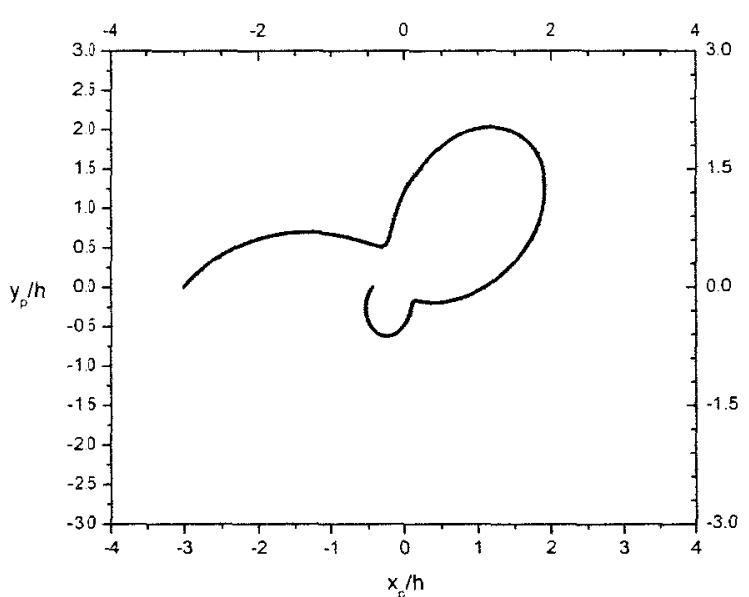

(c) $t_{y}=-0.5$

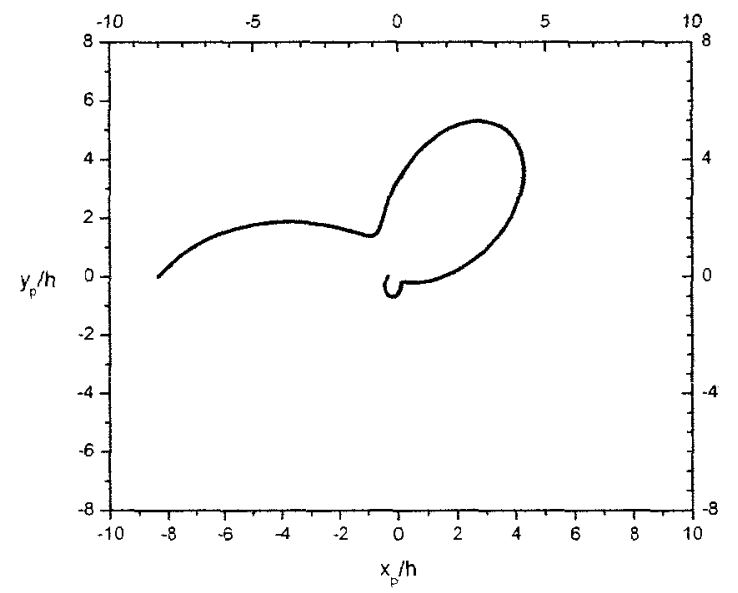

(b) $t_{y}=-0.75$

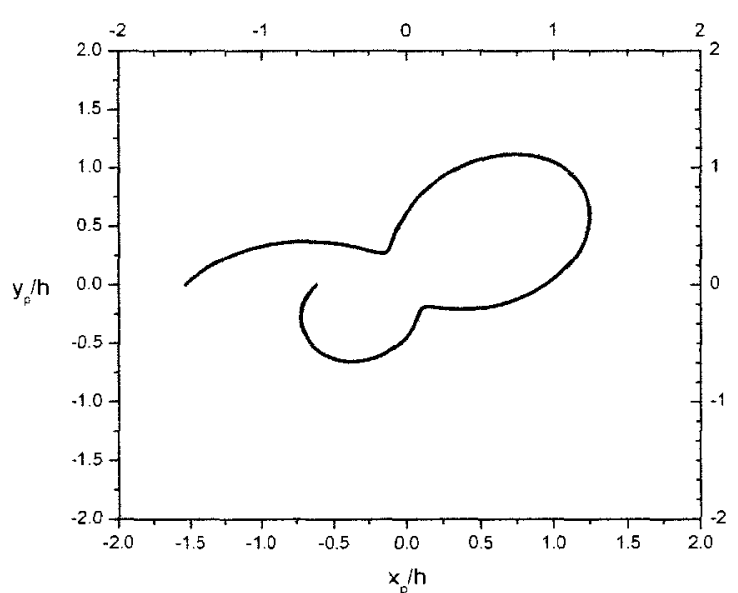

(d) $t_{y}=-0.25$

Figure 4.6: Plastic zone maps for $\phi=50^{\circ}$ (a)-(d) 


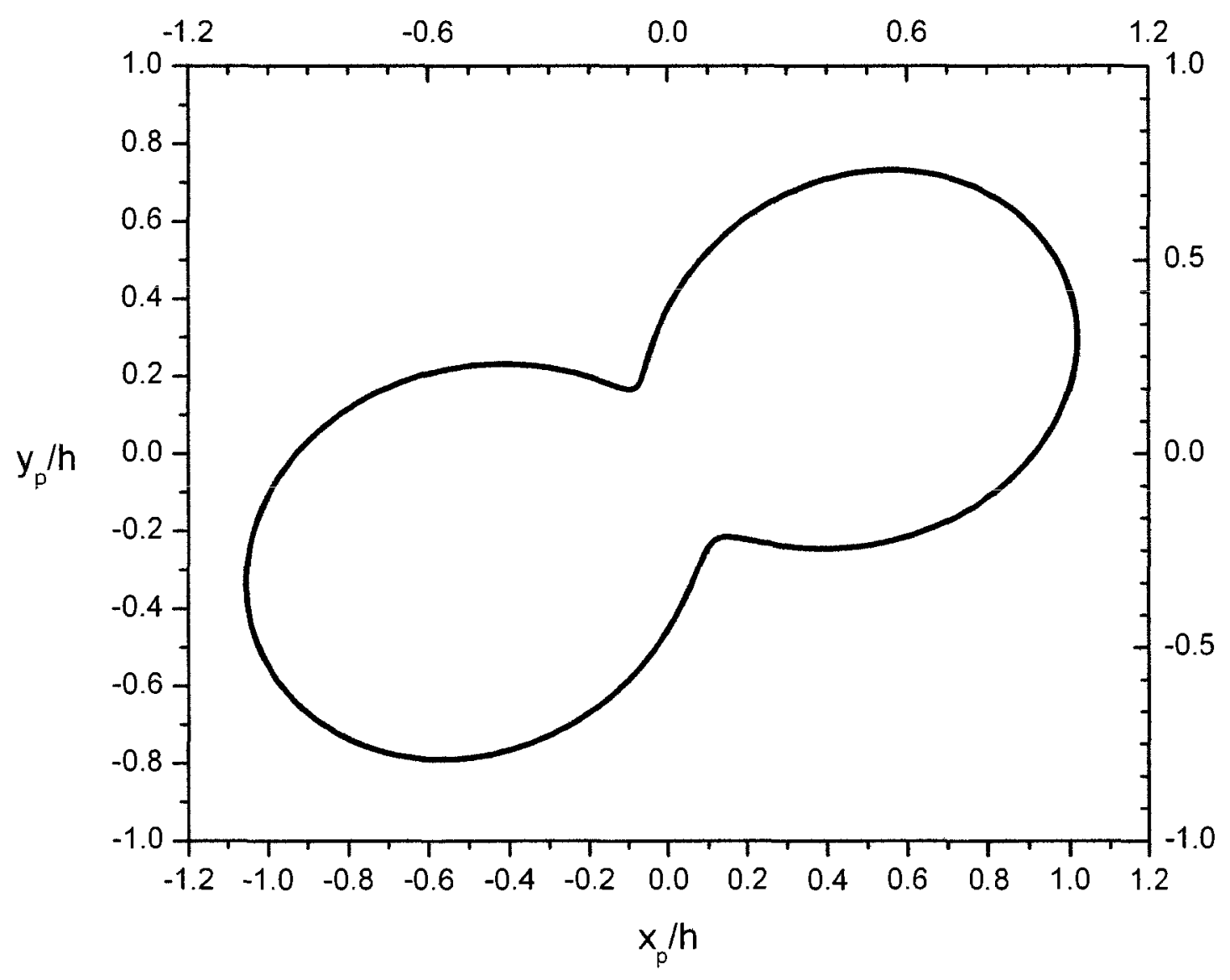

(e) $t_{y}=0$

Figure 4.6: Plastic zone maps for $\phi=50^{\circ}$ (e) 


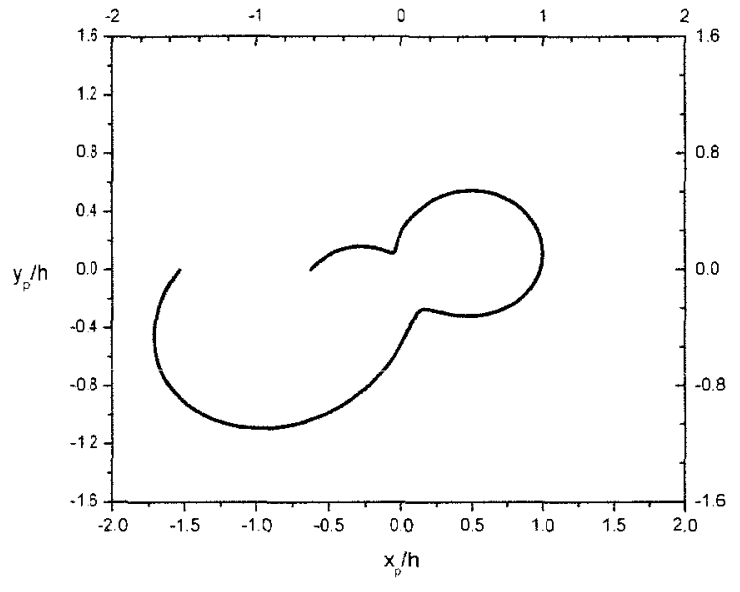

(f) $t_{y}=0.25$

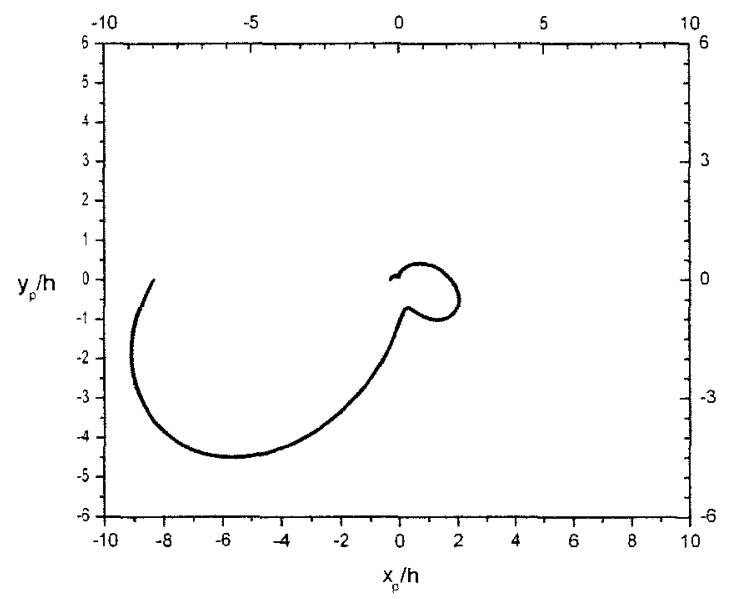

(h) $t_{y}=0.75$

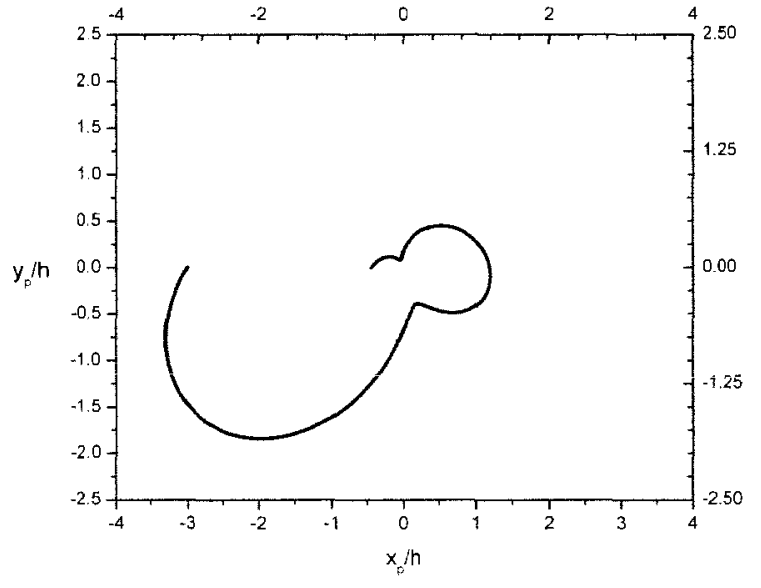

(g) $t_{y}=0.5$

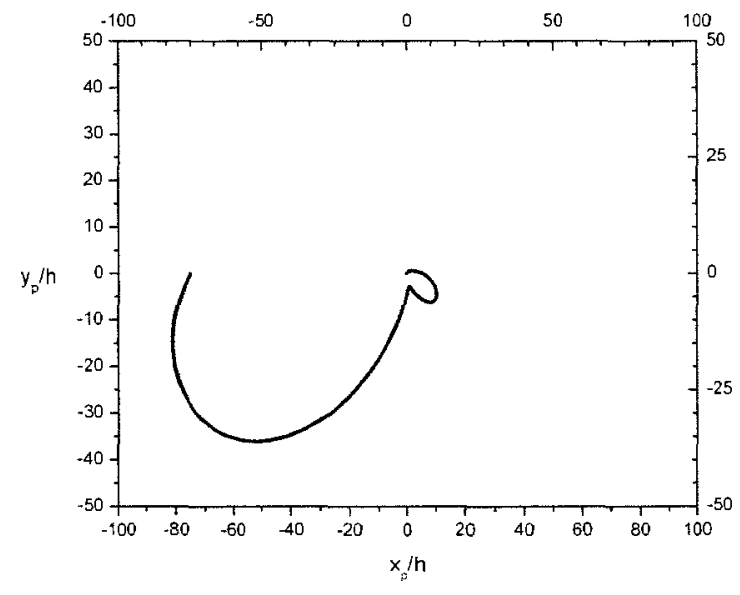

(i) $t_{y}=1$

Figure 4.6: Plastic zone maps for $\phi=50^{\circ}$ (f)-(i) 


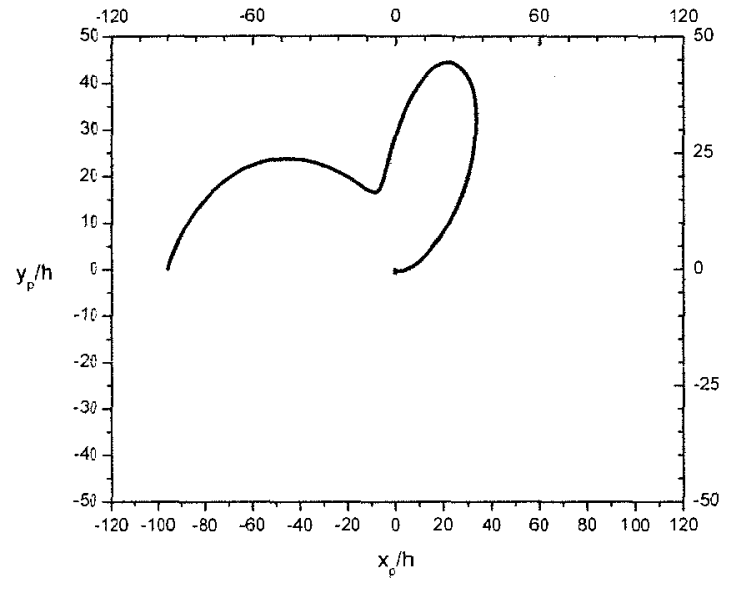

(a) $t_{y}=-1$

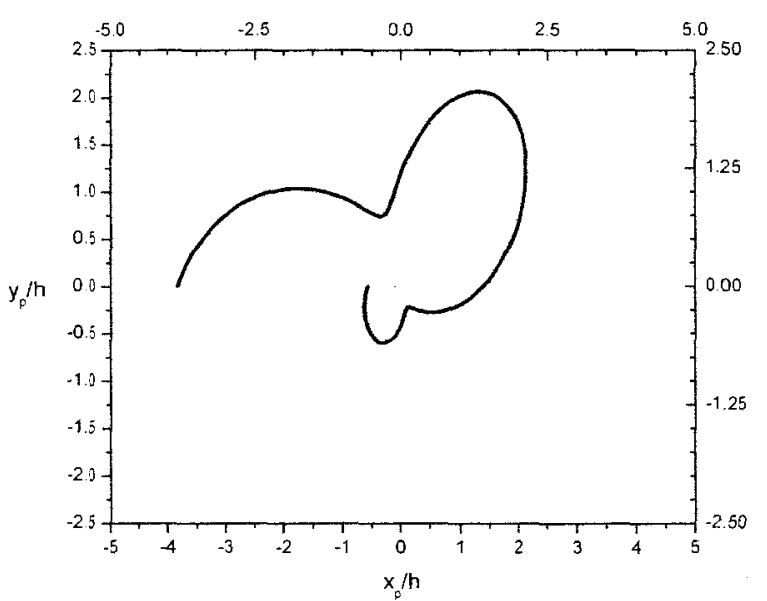

(c) $t_{y}=-0.5$

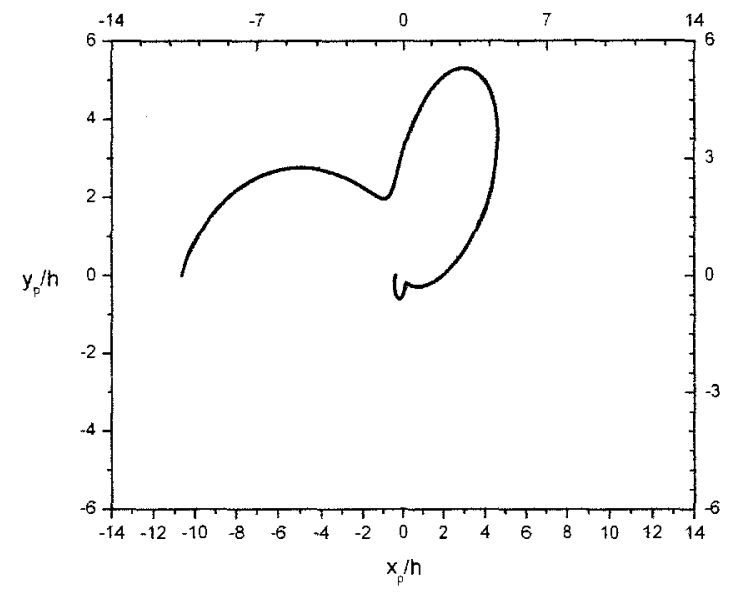

(b) $t_{y}=-0.75$

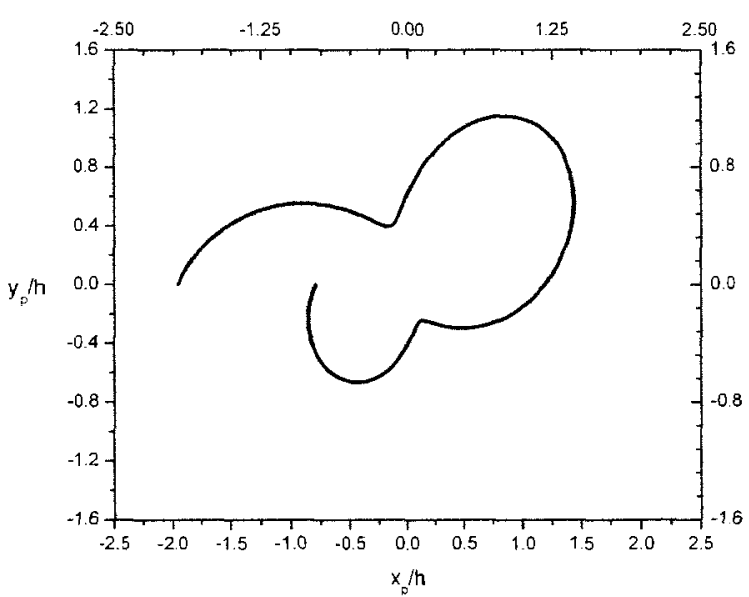

(d) $t_{y}=-0.25$

Figure 4.7: Plastic zone maps for $\phi=60^{\circ}$ (a)-(d) 


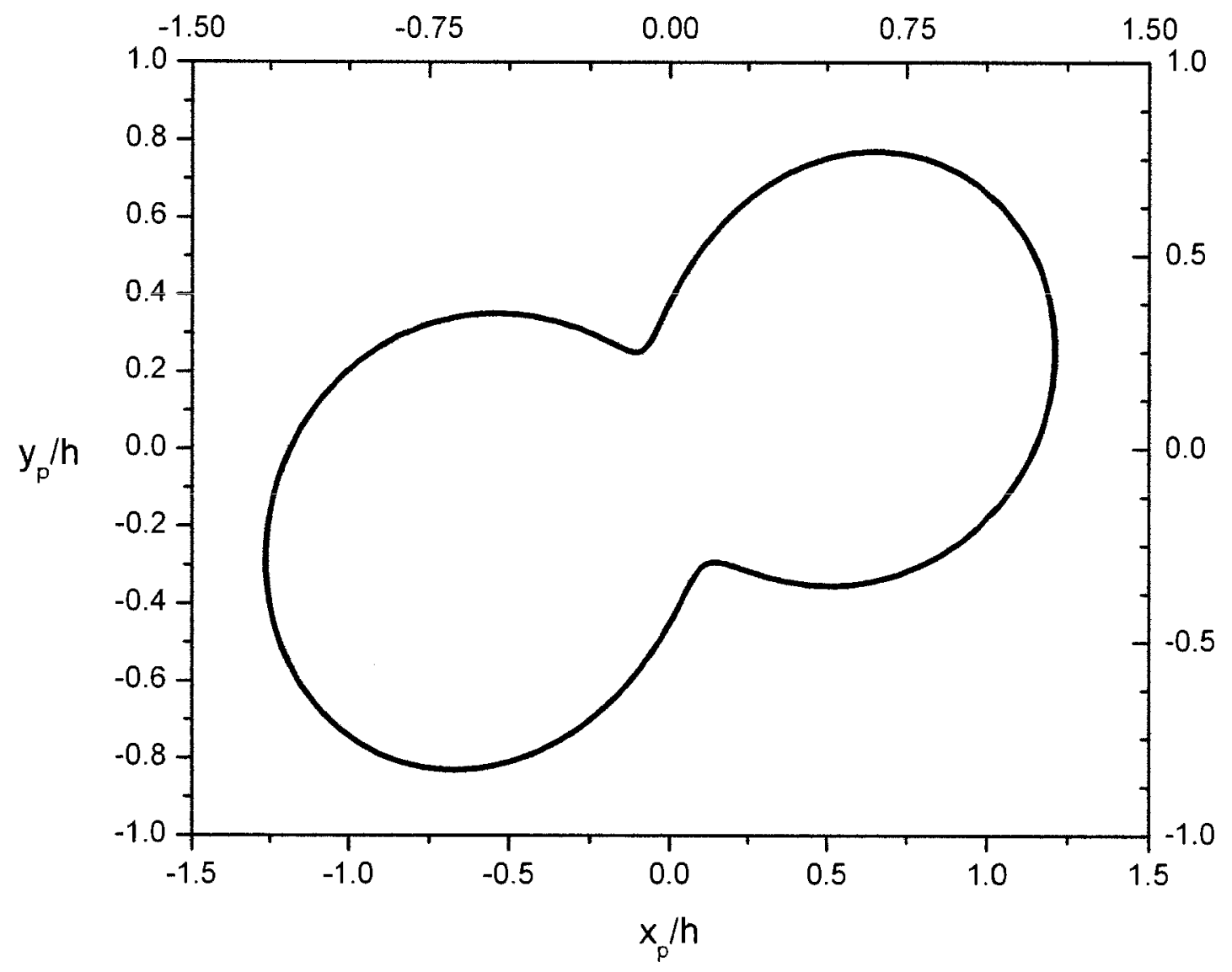

(e) $t_{y}=0$

Figure 4.7: Plastic zone maps for $\phi=60^{\circ}$ (e) 


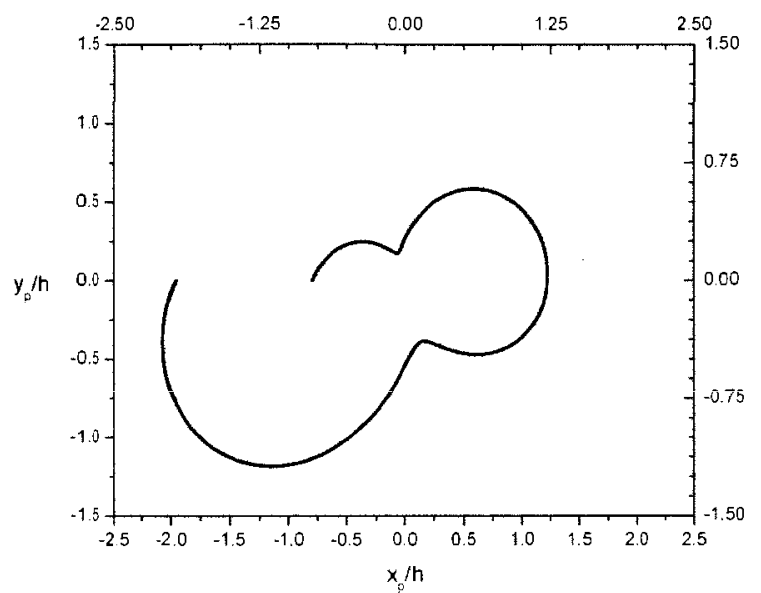

(f) $t_{y}=0.25$

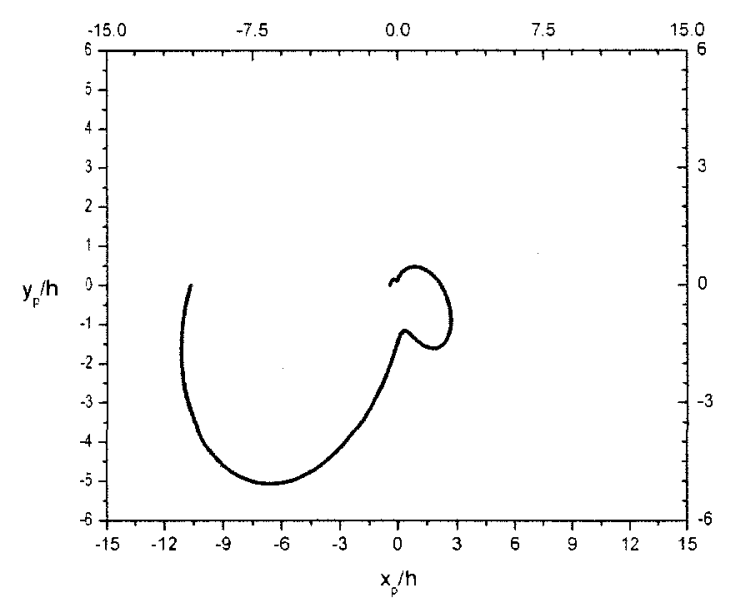

(h) $t_{y}=0.75$

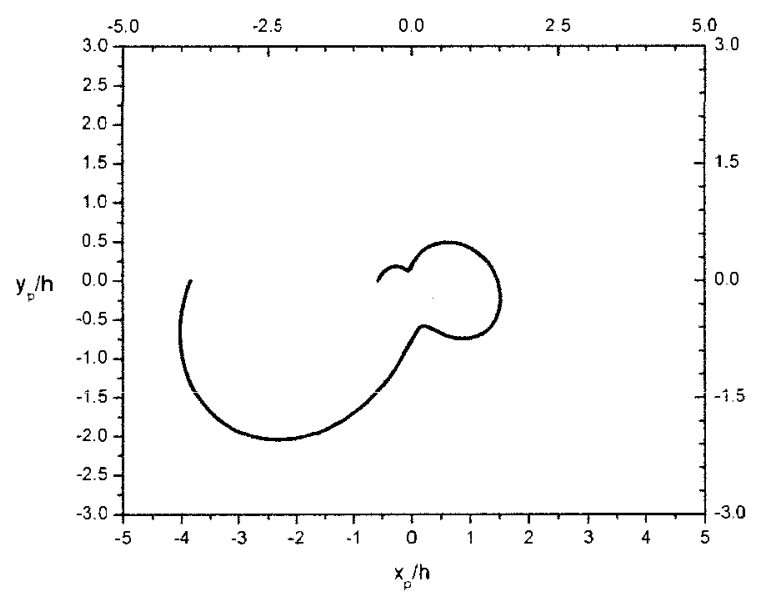

(g) $t_{y}=0.5$

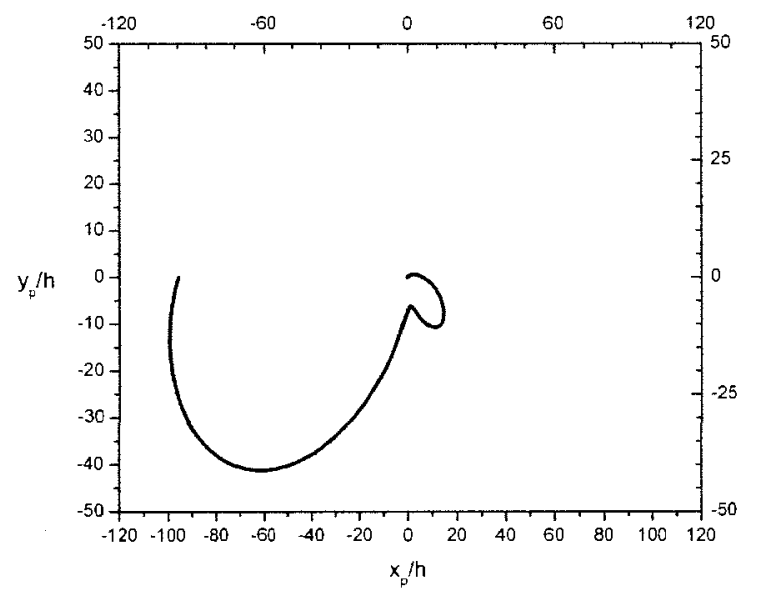

(i) $t_{y}=1$

Figure 4.7: Plastic zone maps for $\phi=60^{\circ}$ (f)-(i) 


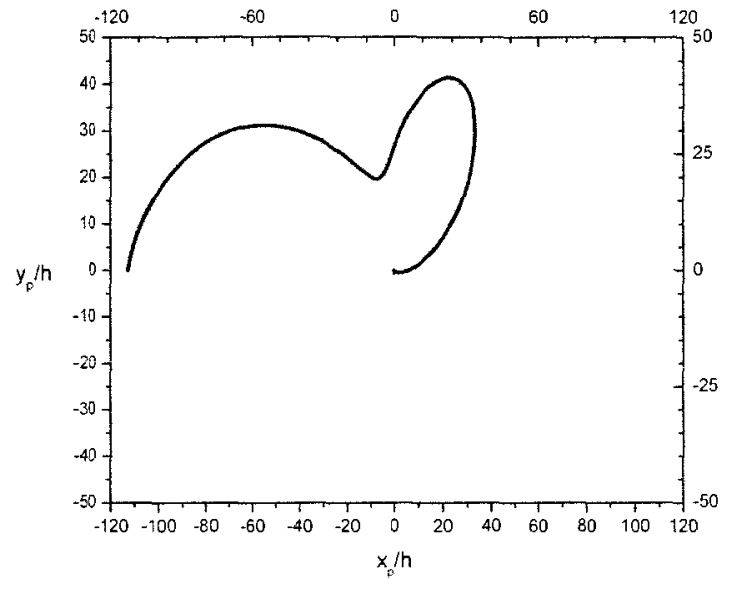

(a) $t_{y}=-1$

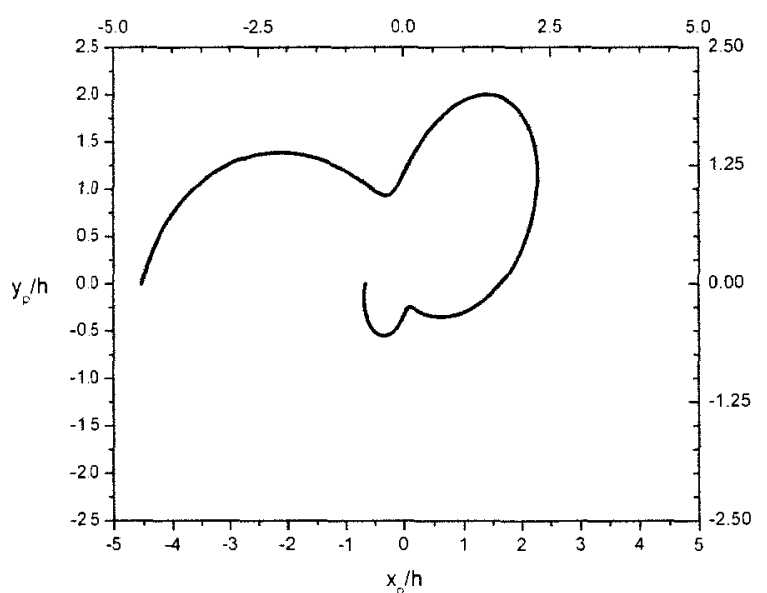

(c) $t_{y}=-0.5$

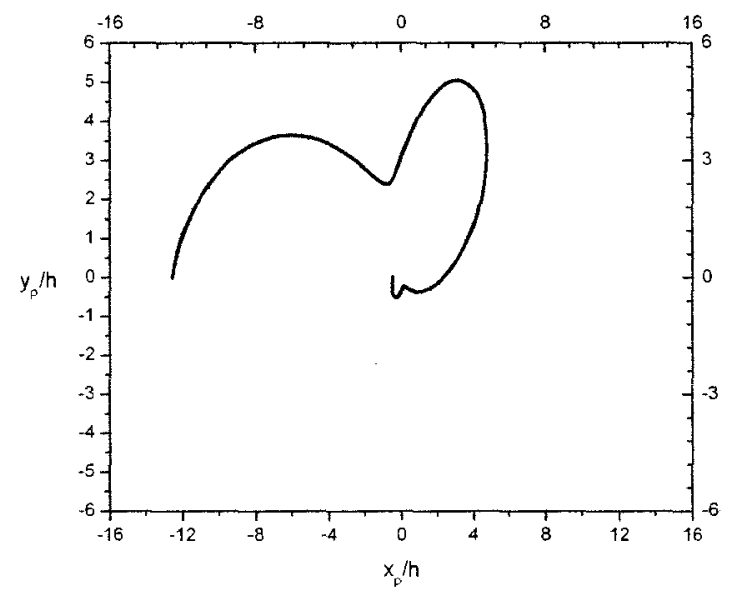

(b) $t_{y}=-0.75$

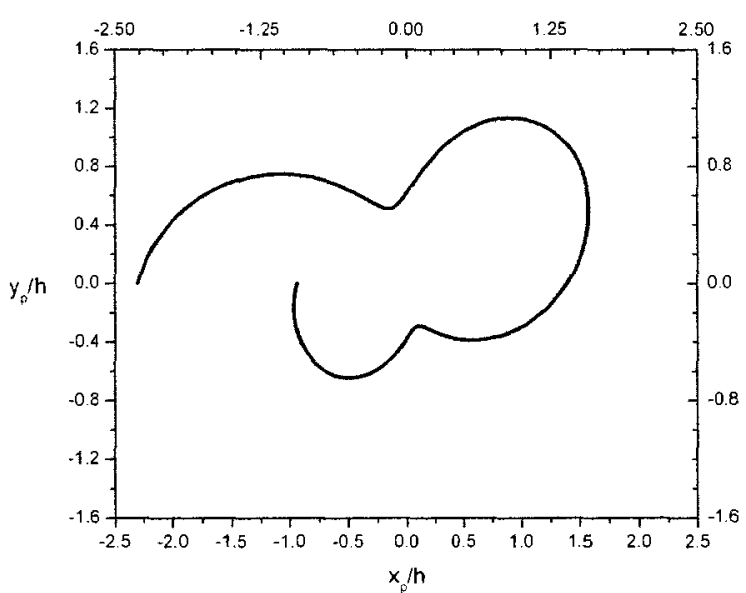

(d) $t_{y}=-0.25$

Figure 4.8: Plastic zone maps for $\phi=70^{\circ}$ (a)-(d) 


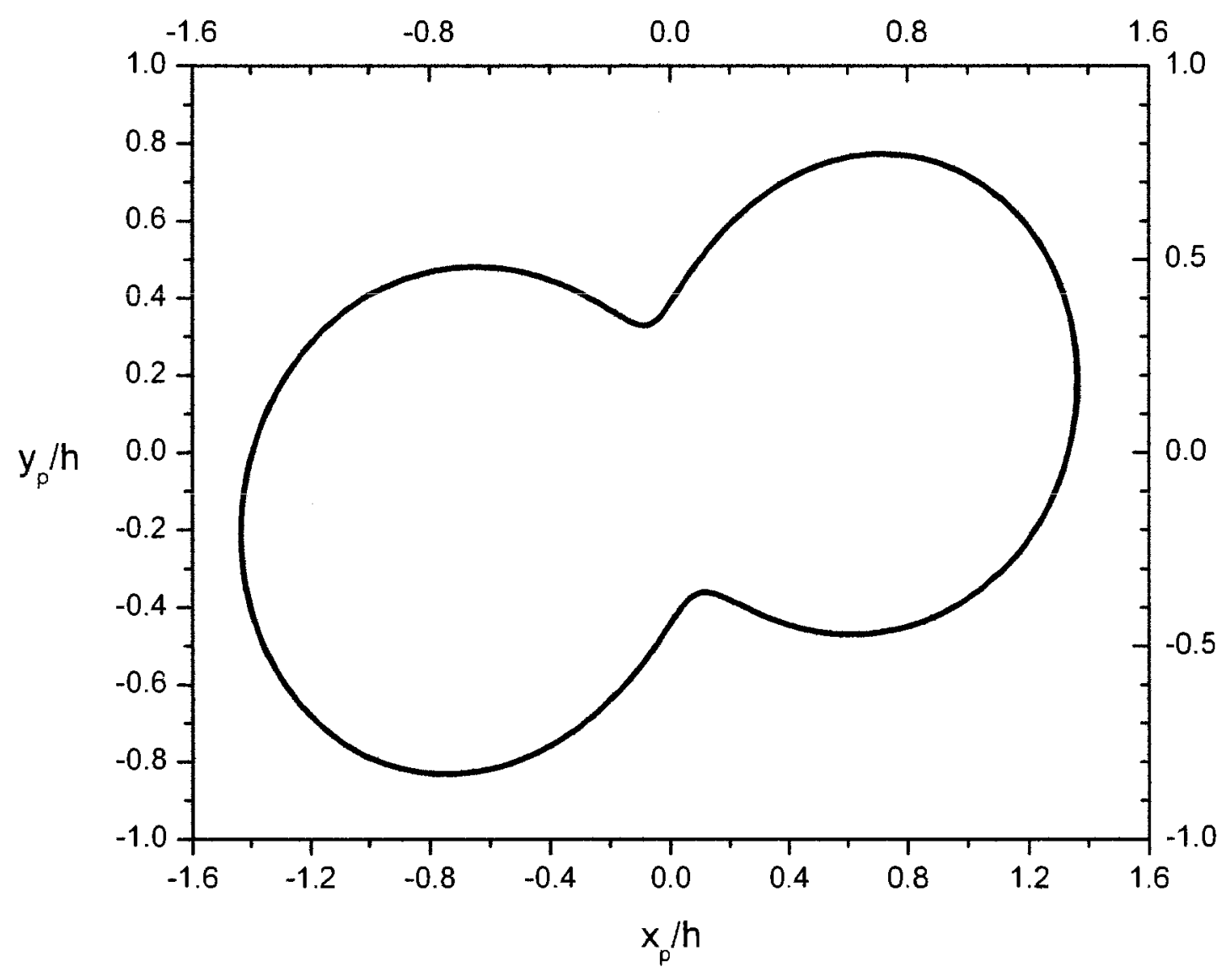

(e) $t_{y}=0$

Figure 4.8: Plastic zone maps for $\phi=70^{\circ}$ (e) 


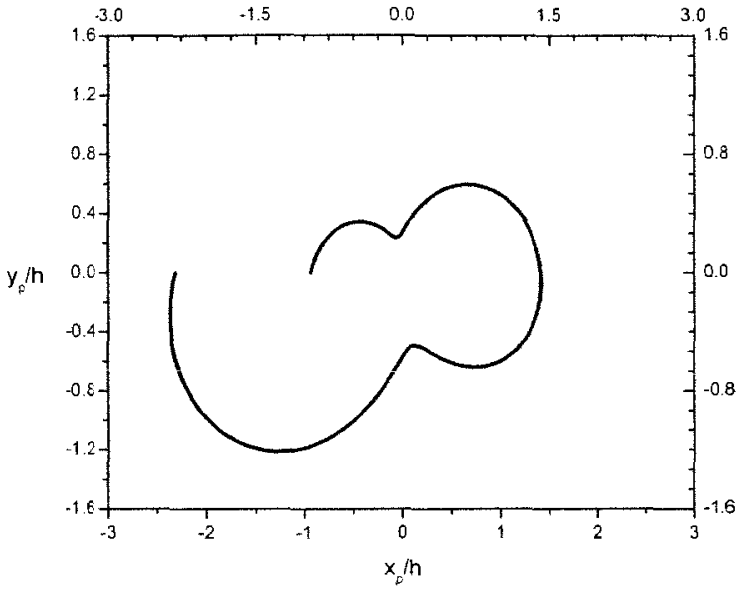

(f) $t_{y}=0.25$

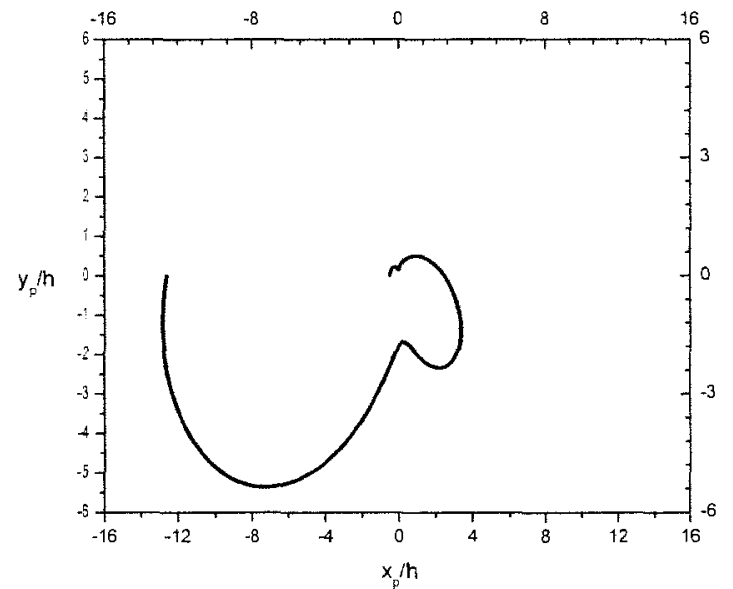

(h) $t_{y}=0.75$

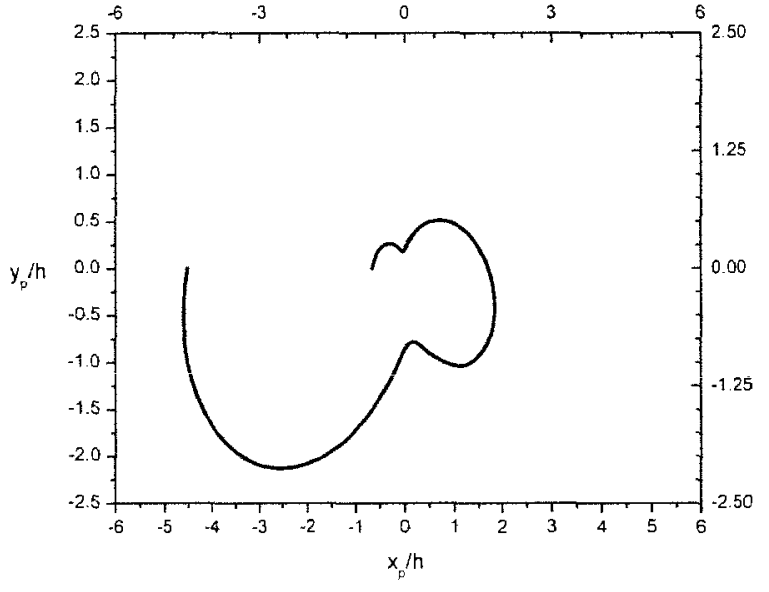

(g) $t_{y}=0.5$

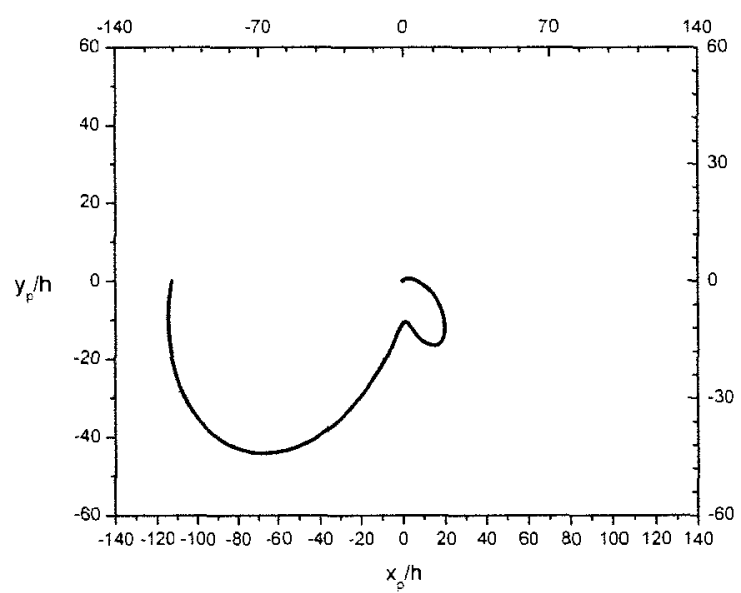

(i) $t_{y}=1$

Figure 4.8: Plastic zone maps for $\phi=70^{\circ}$ (f)-(i) 


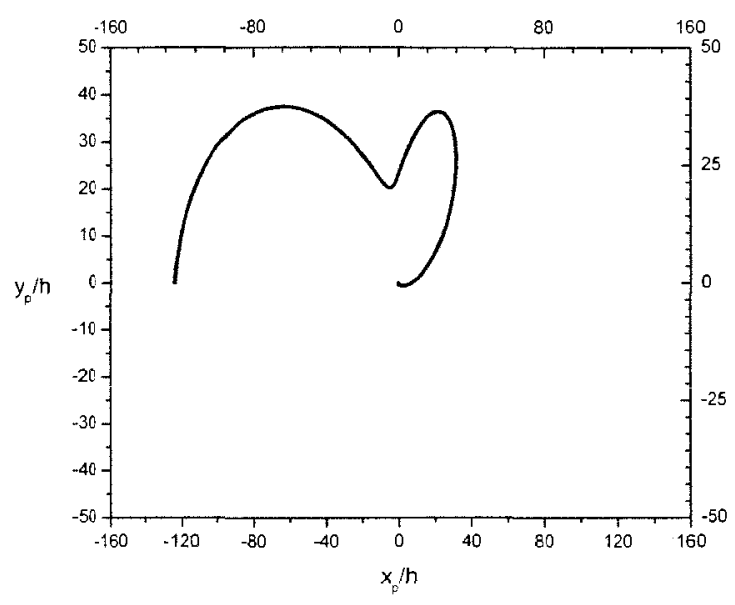

(a) $t_{y}=-1$

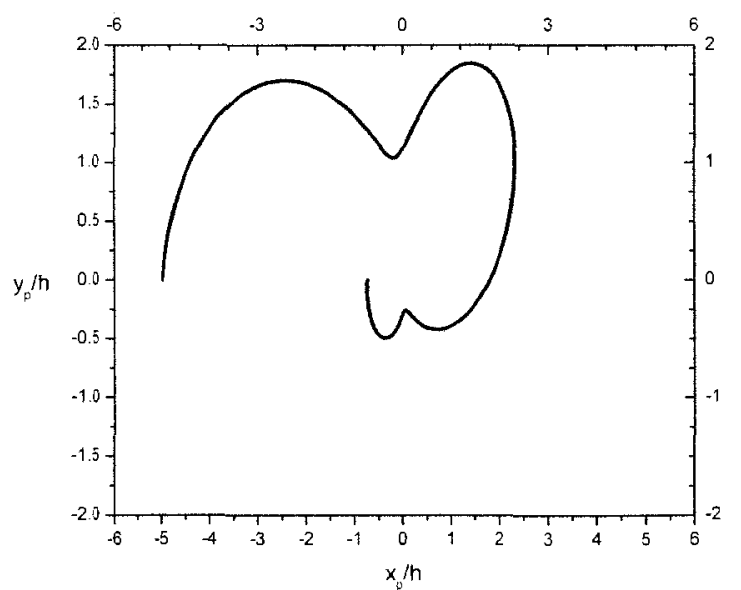

(c) $t_{y}=-0.5$

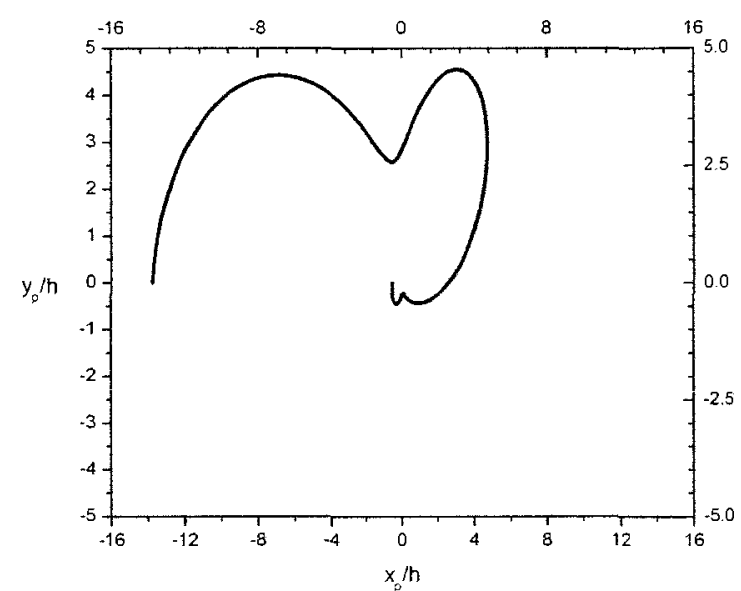

(b) $t_{y}=-0.75$

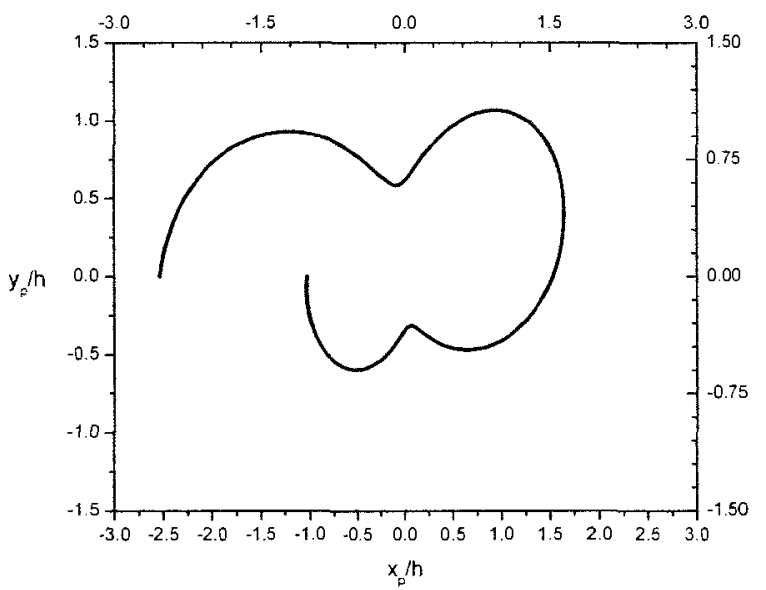

(d) $t_{y}=-0.25$

Figure 4.9: Plastic zone maps for $\phi=80^{\circ}$ (a)-(d) 


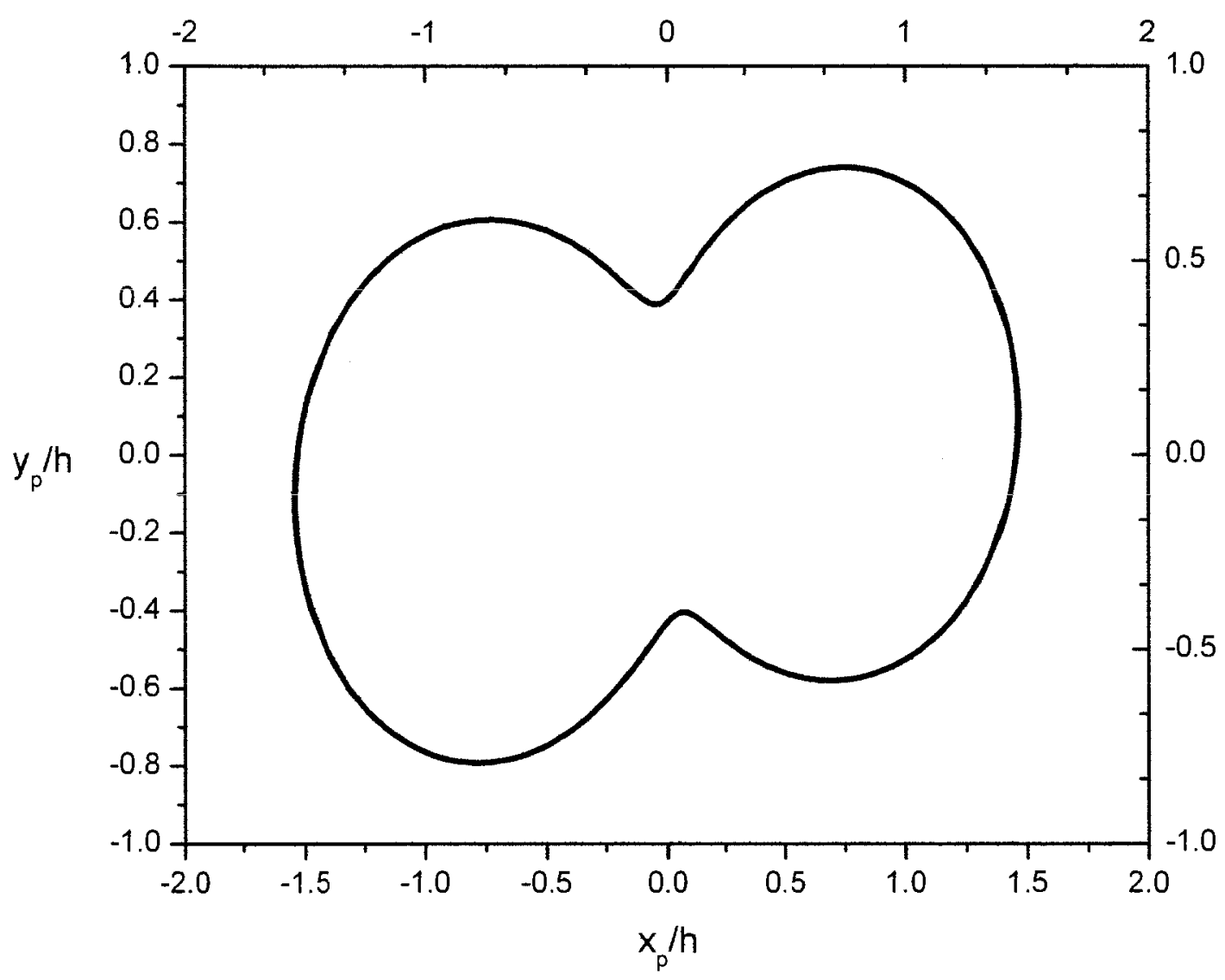

(e) $t_{y}=0$

Figure 4.9: Plastic zone maps for $\phi=80^{\circ}$ (e) 


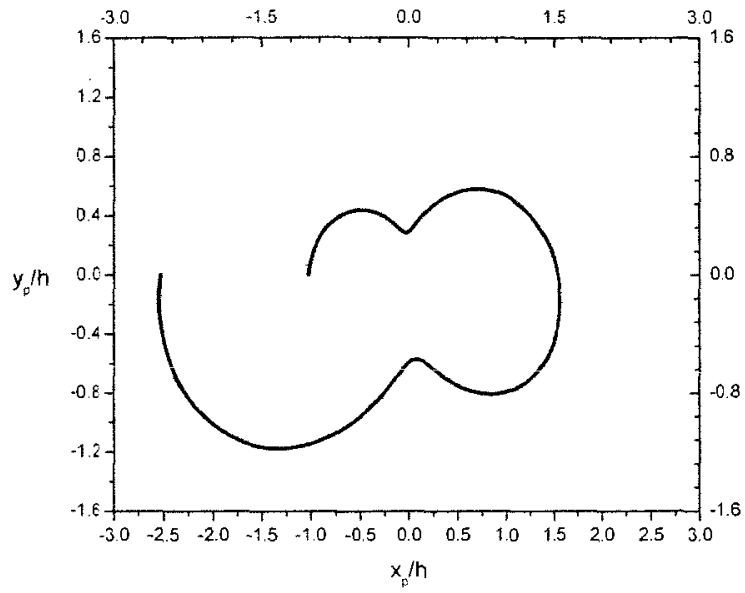

(f) $t_{y}=0.25$

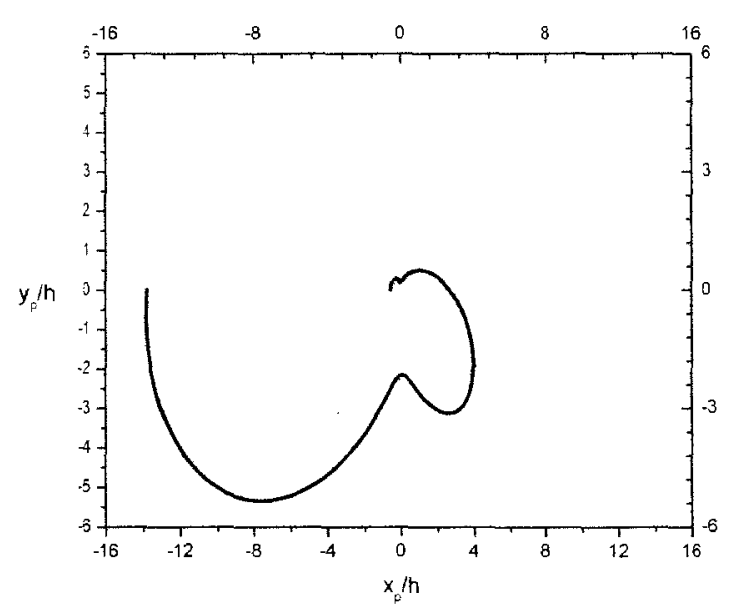

(h) $t_{y}=0.75$

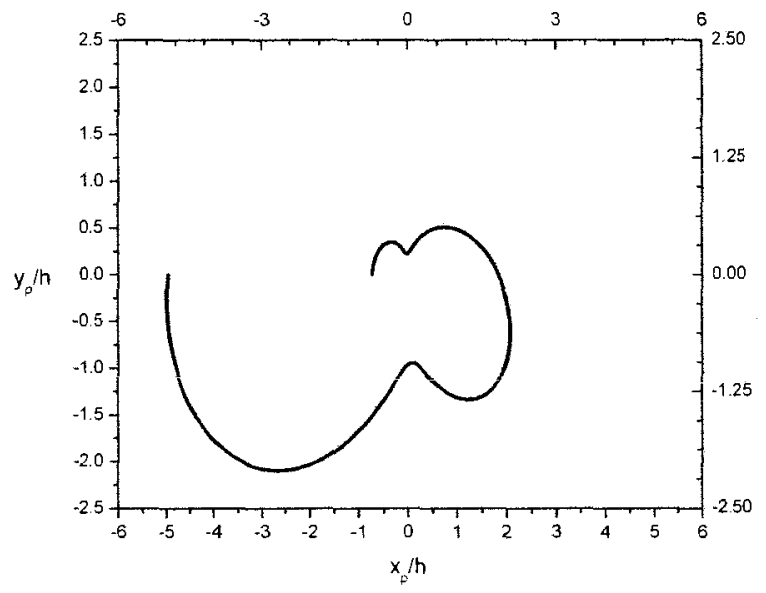

(g) $t_{y}=0.5$

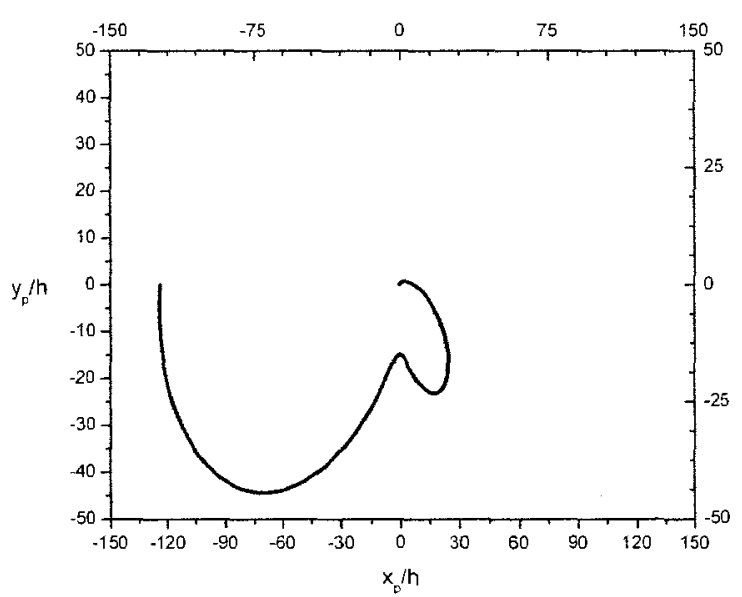

(i) $t_{y}=1$

Figure 4.9: Plastic zone maps for $\phi=80^{\circ}$ (f)-(i) 


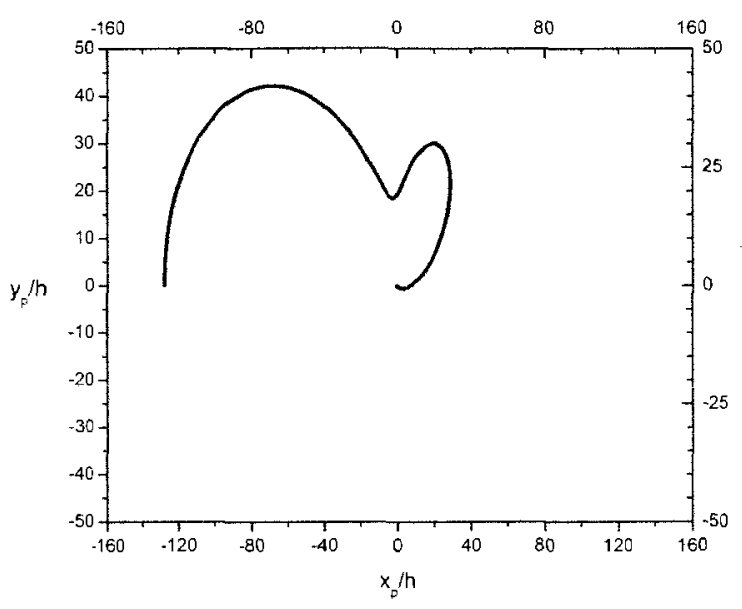

(a) $t_{y}=-1$

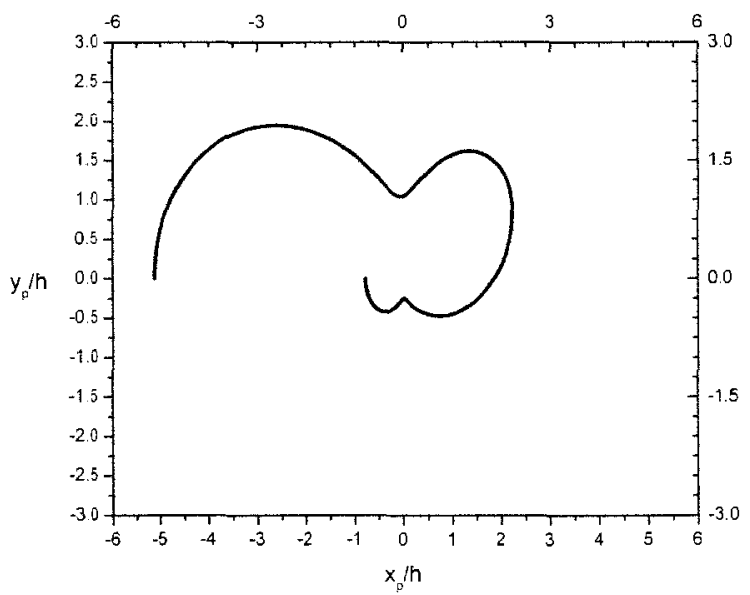

(c) $t_{y}=-0.5$

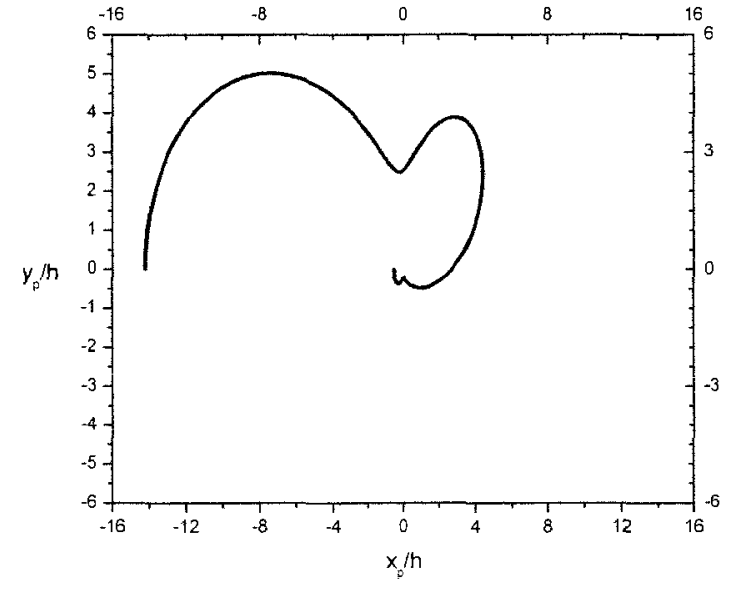

(b) $t_{y}=-0.75$

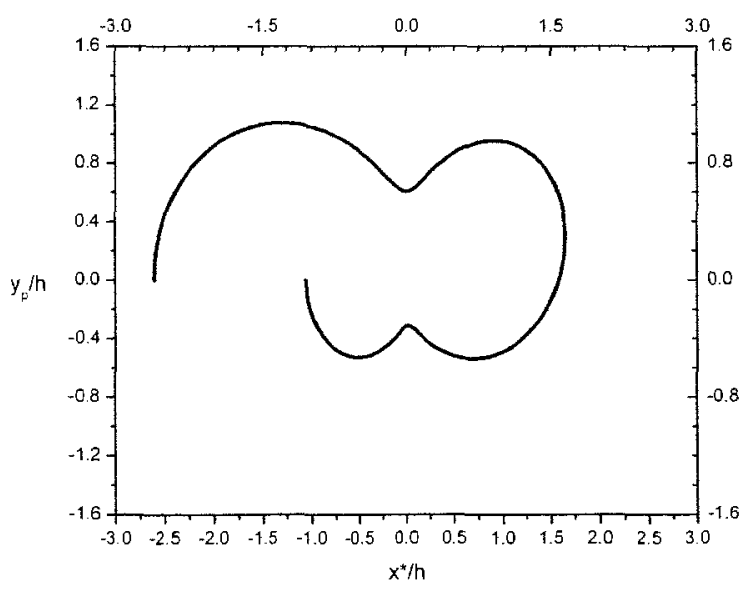

(d) $t_{y}=-0.25$

Figure 4.10: Plastic zone maps for $\phi=90^{\circ}$ (a)-(d) 


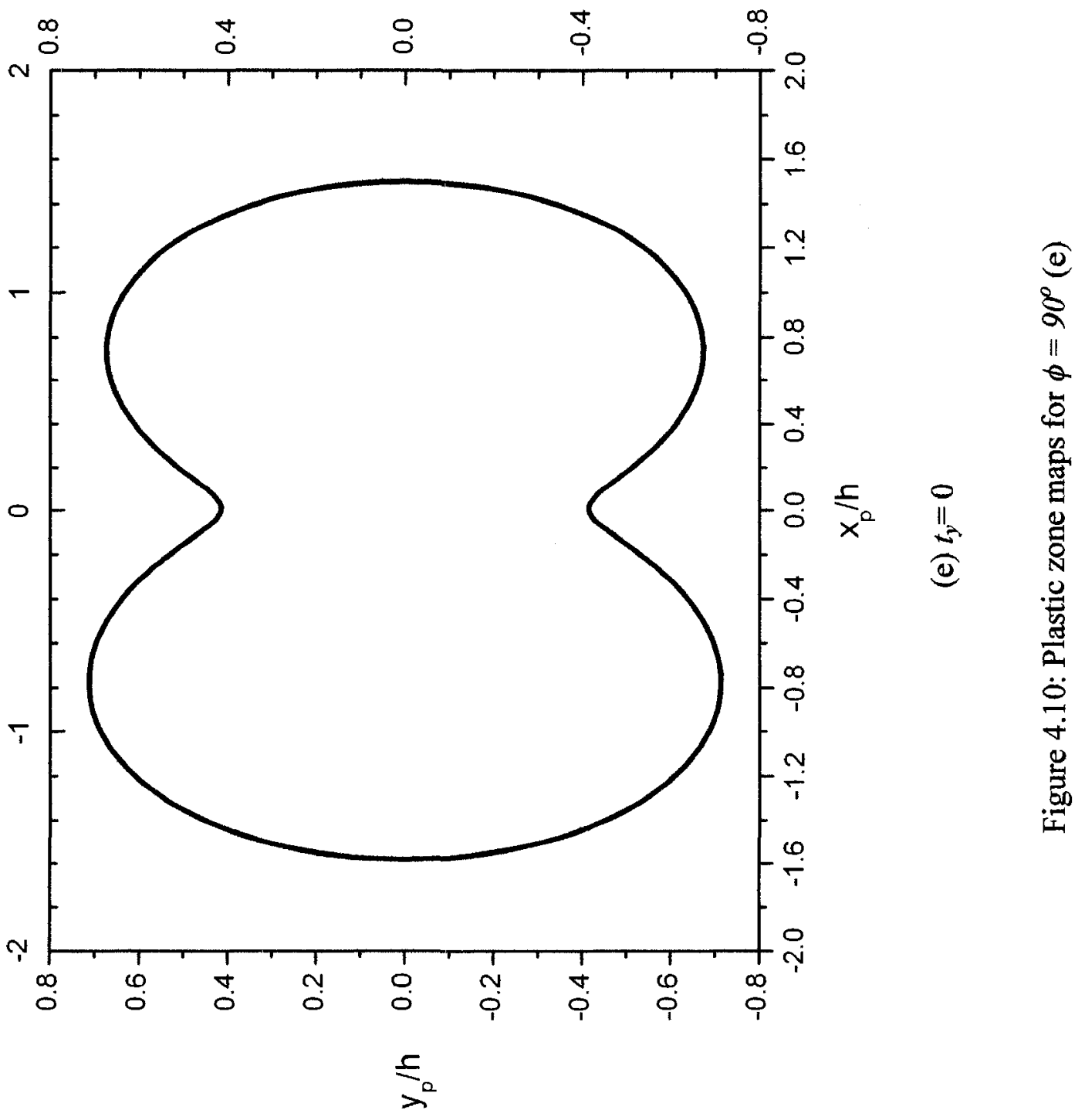




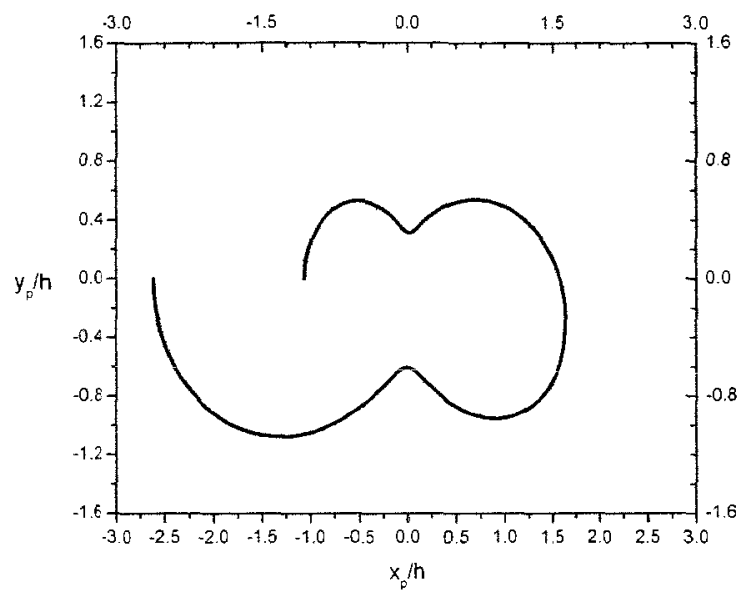

(f) $t_{y}=0.25$

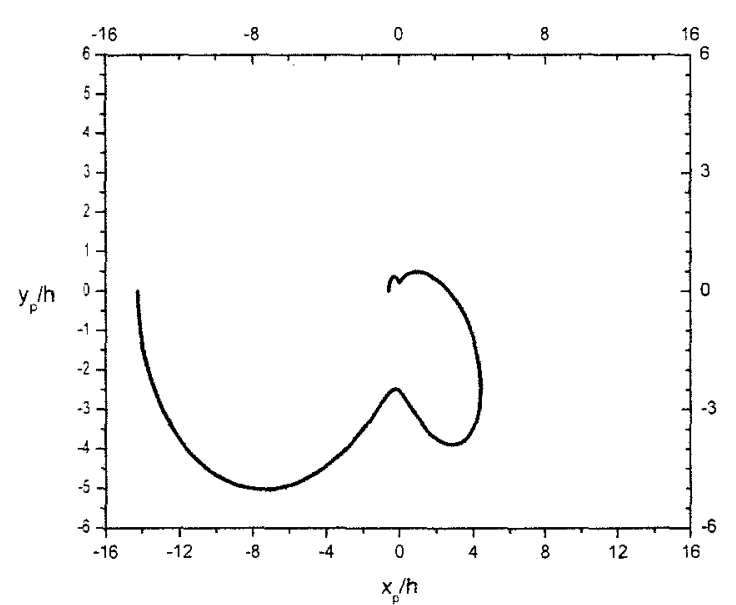

(h) $t_{y}=0.75$

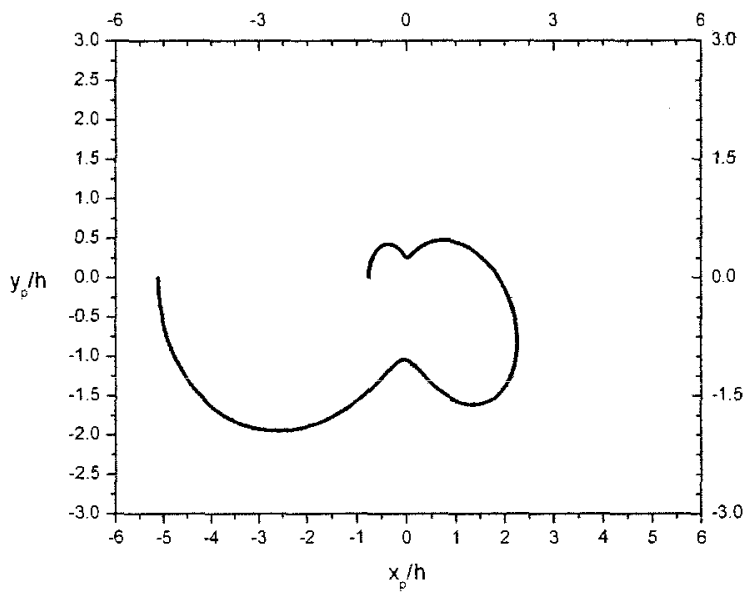

(g) $t_{y}=0.5$

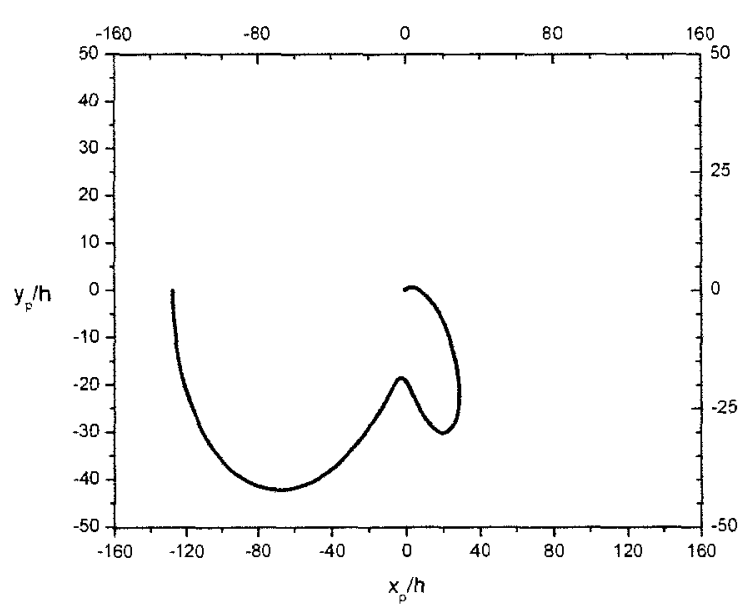

(i) $t_{y}=1$

Figure 4.10: Plastic zone maps for $\phi=90^{\circ}$ (f)-(i) 


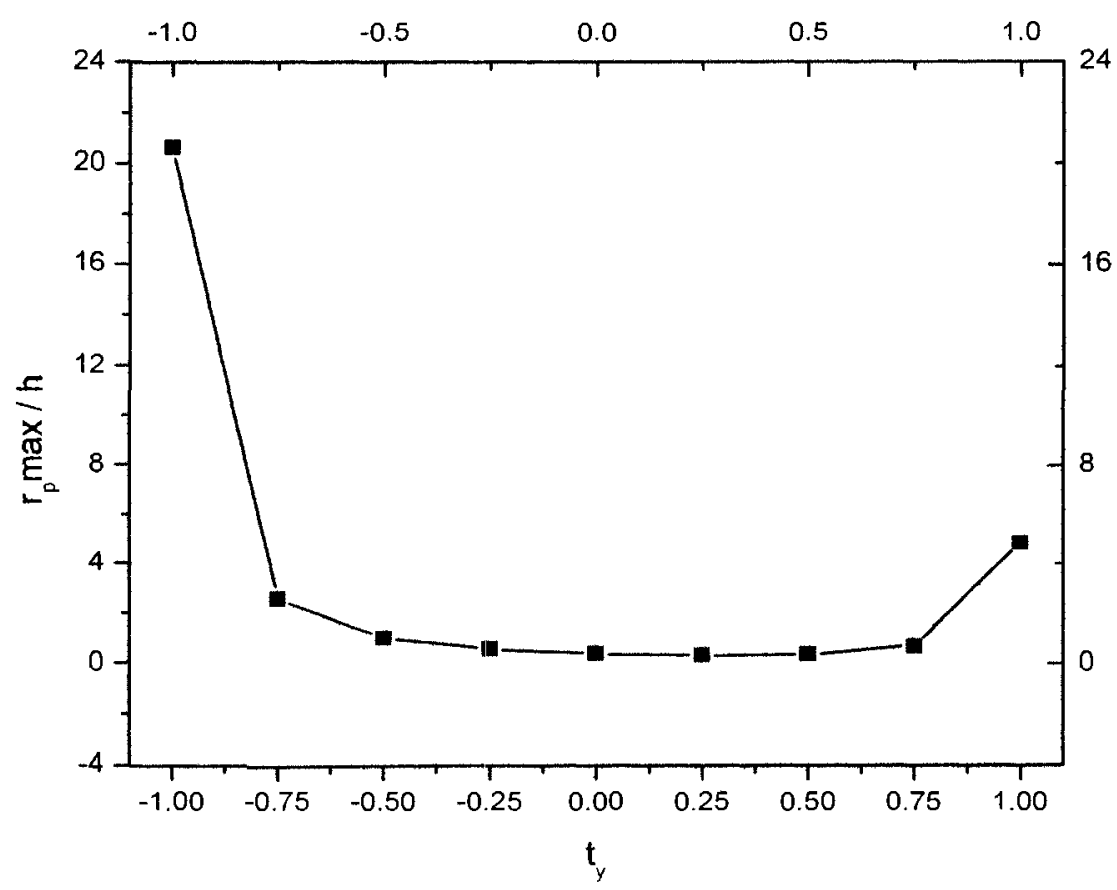

(a) $\phi=0^{\circ}$

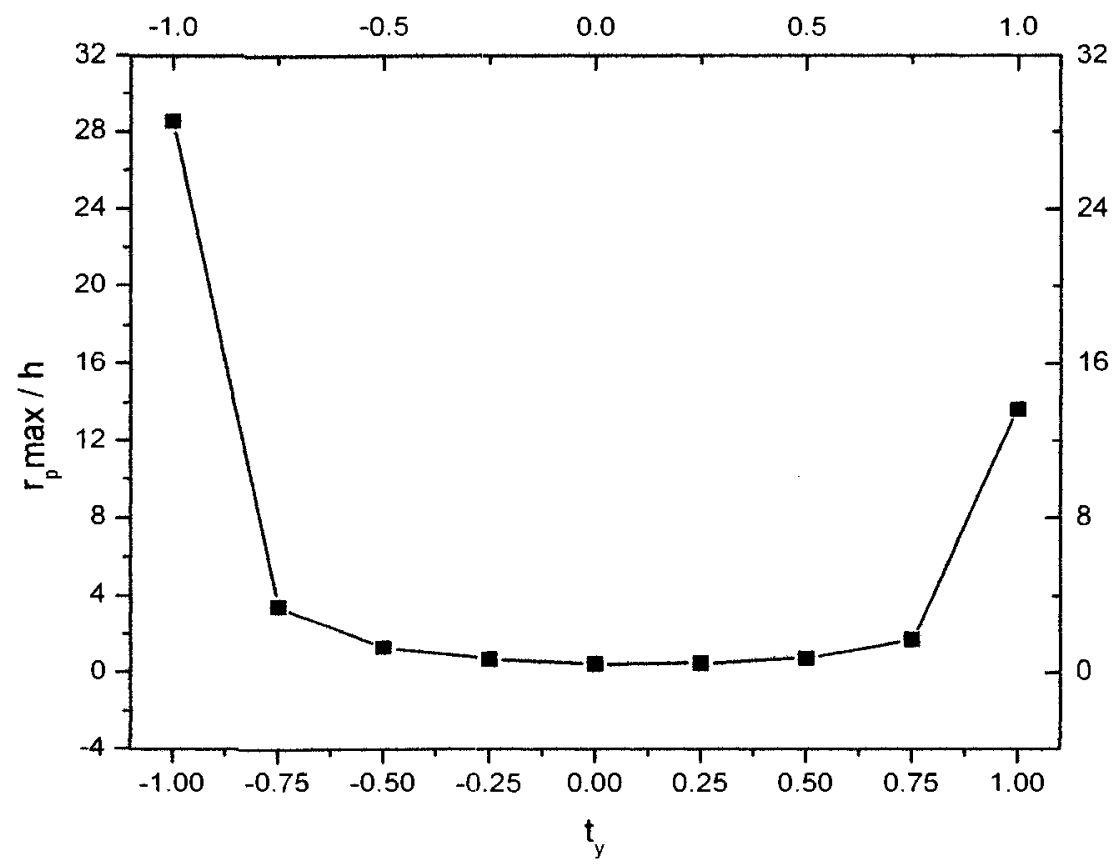

(b) $\phi=10^{\circ}$

Figure 4.11: Graphs of maximum plastic zone size as a function of the normalized Tstress for various phase angles (a)-(b) 


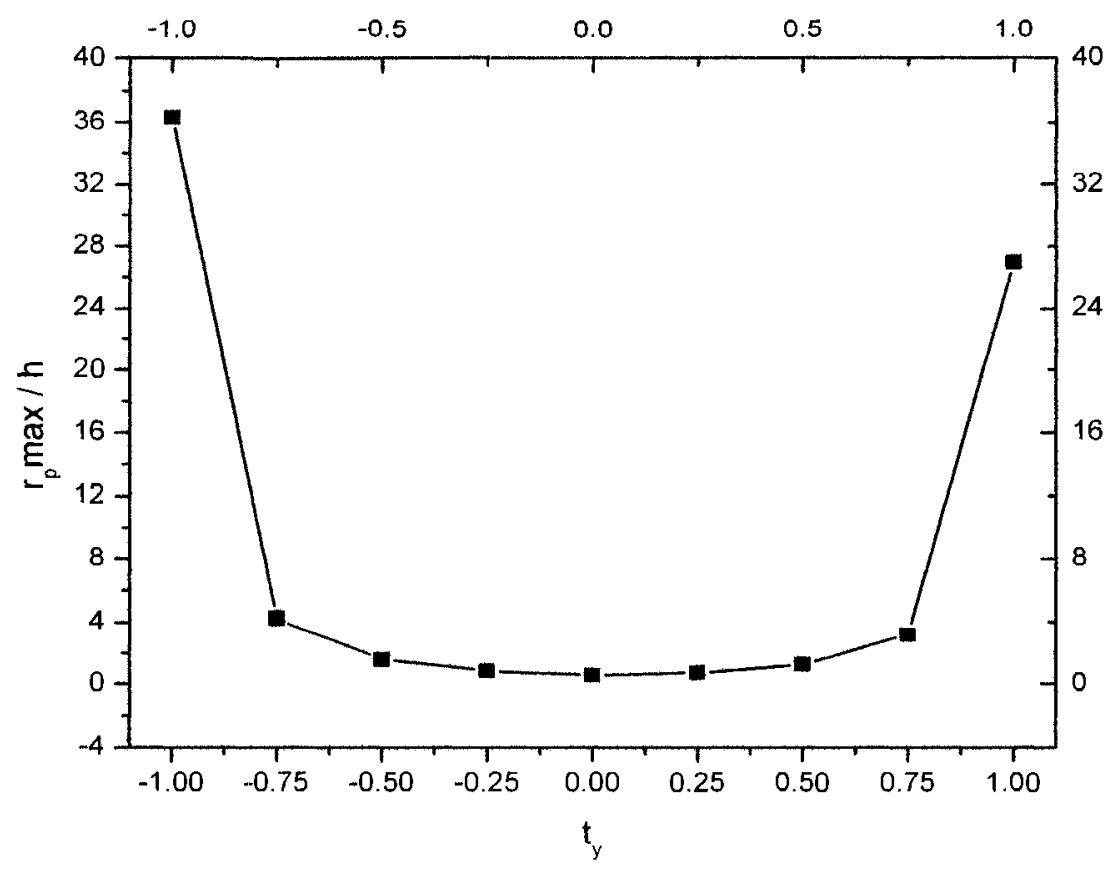

(c) $\phi=20^{\circ}$

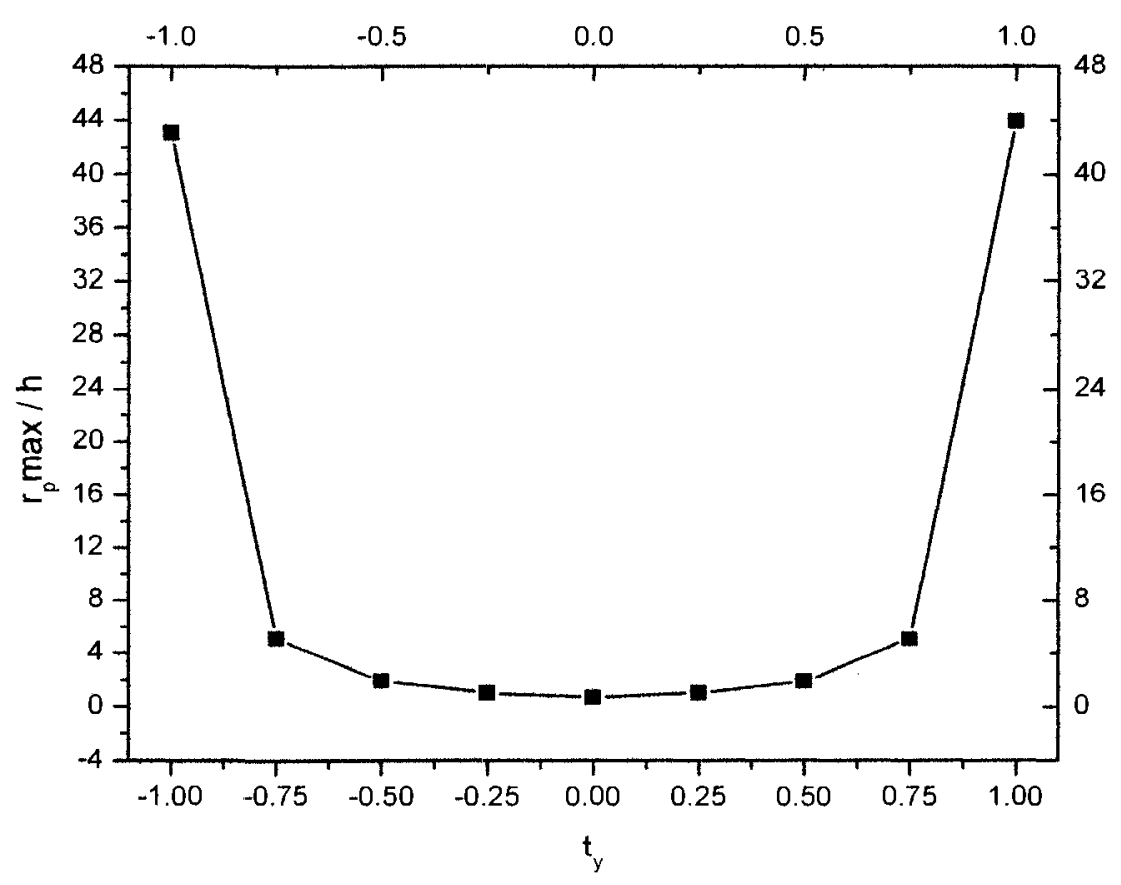

(d) $\phi=30^{\circ}$

Figure 4.11: Graphs of maximum plastic zone size as a function of the normalized Tstress for various phase angles (c)-(d) 


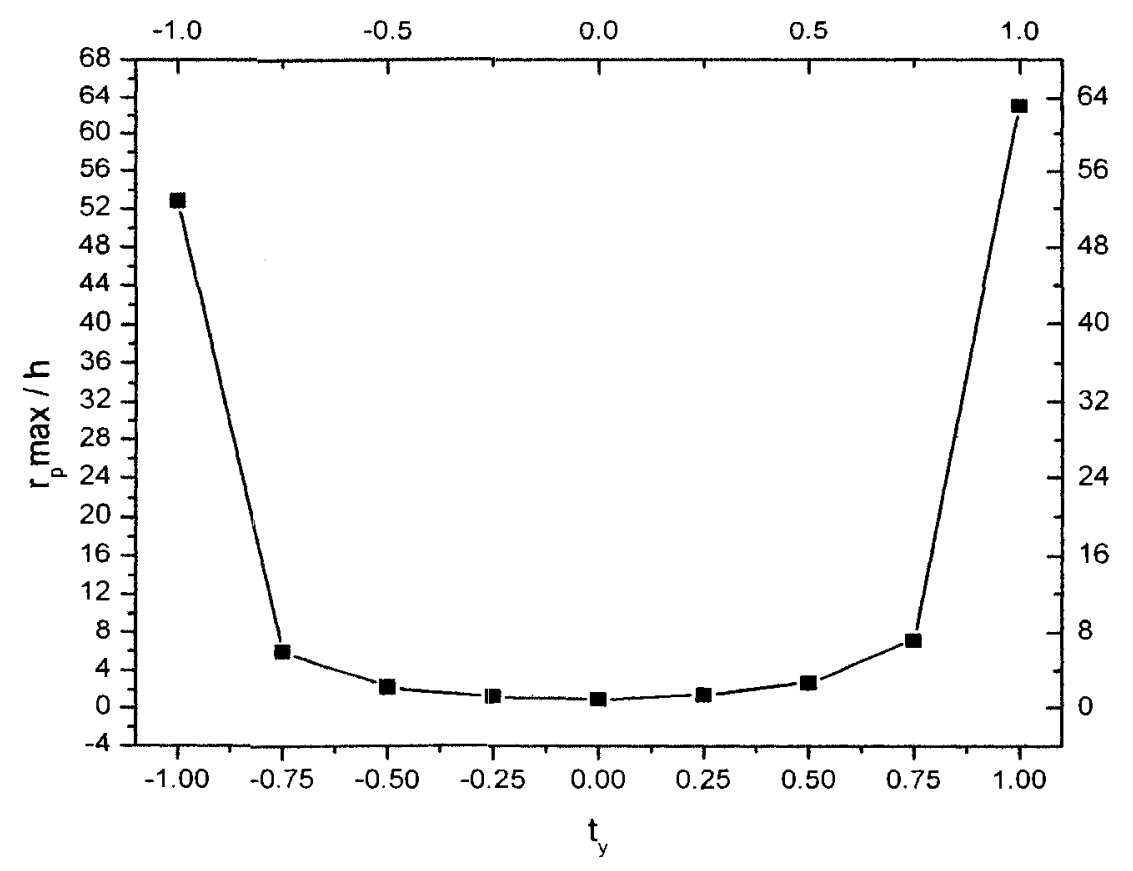

(e) $\phi=40^{\circ}$

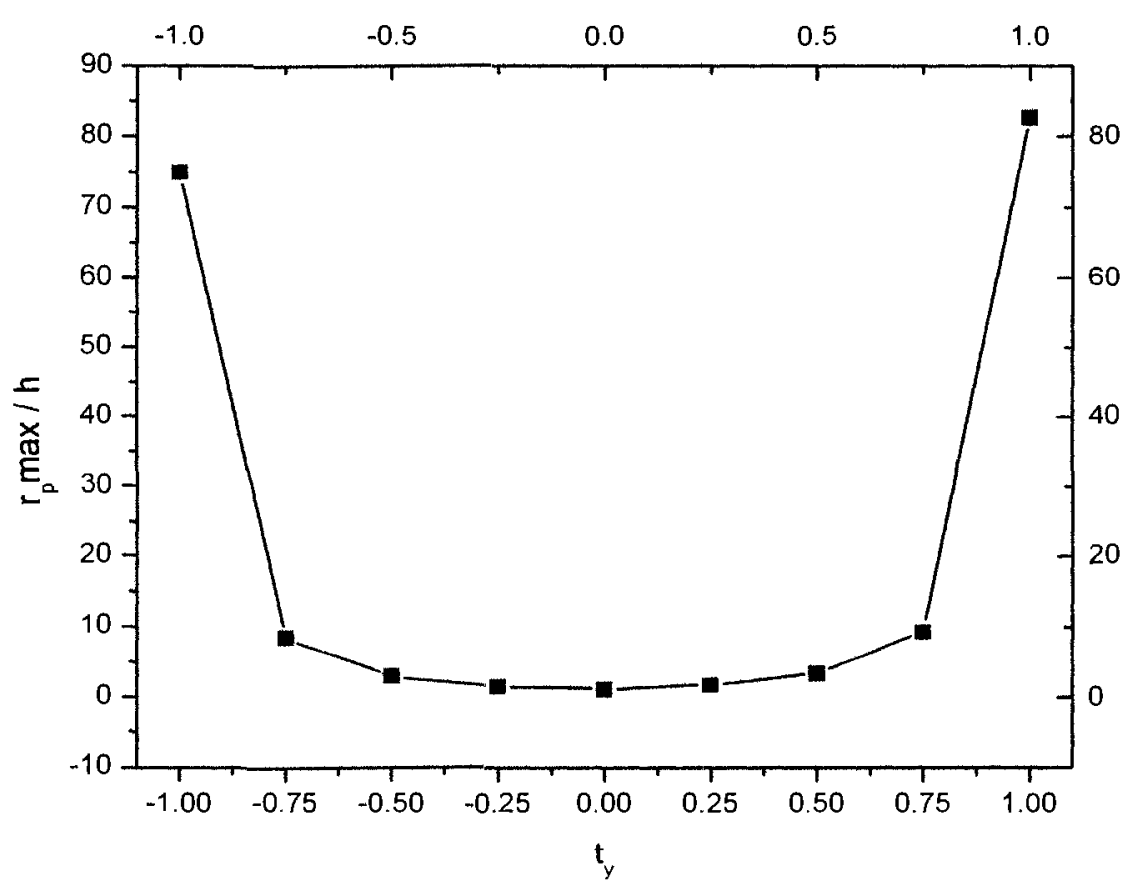

(f) $\phi=50^{\circ}$

Figure 4.11: Graphs of maximum plastic zone size as a function of the normalized Tstress for various phase angles (e)-(f) 


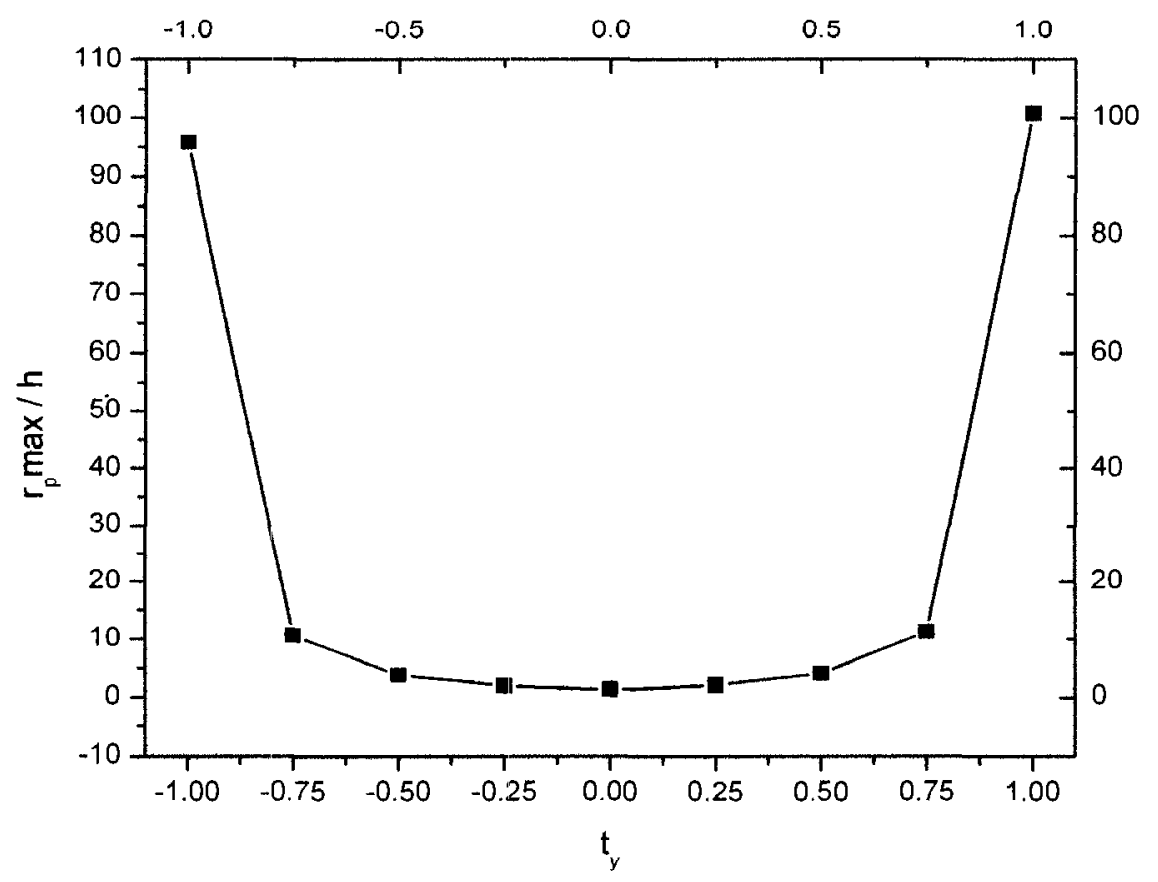

(g) $\phi=60^{\circ}$

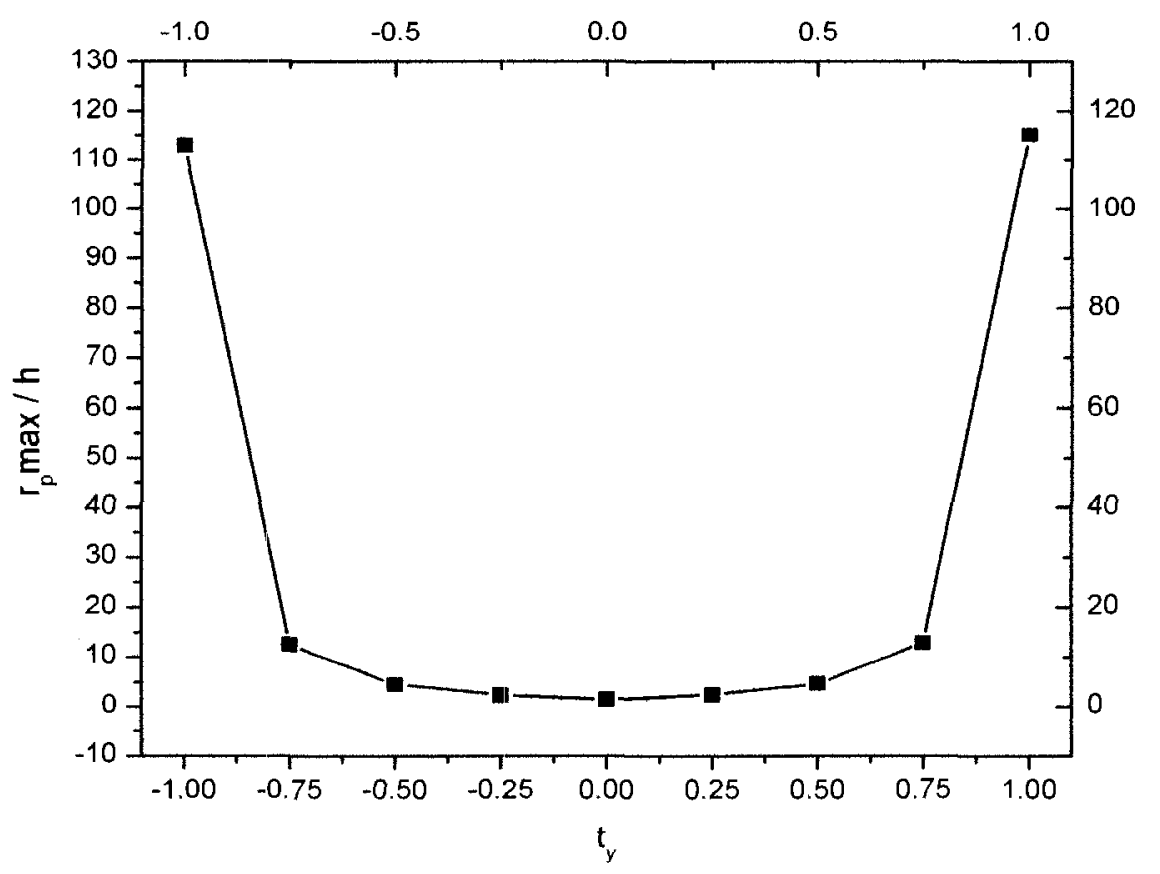

(h) $\phi=70^{\circ}$

Figure 4.11: Graphs of maximum plastic zone size as a function of the normalized Tstress for various phase angles $(\mathrm{g})-(\mathrm{h})$ 


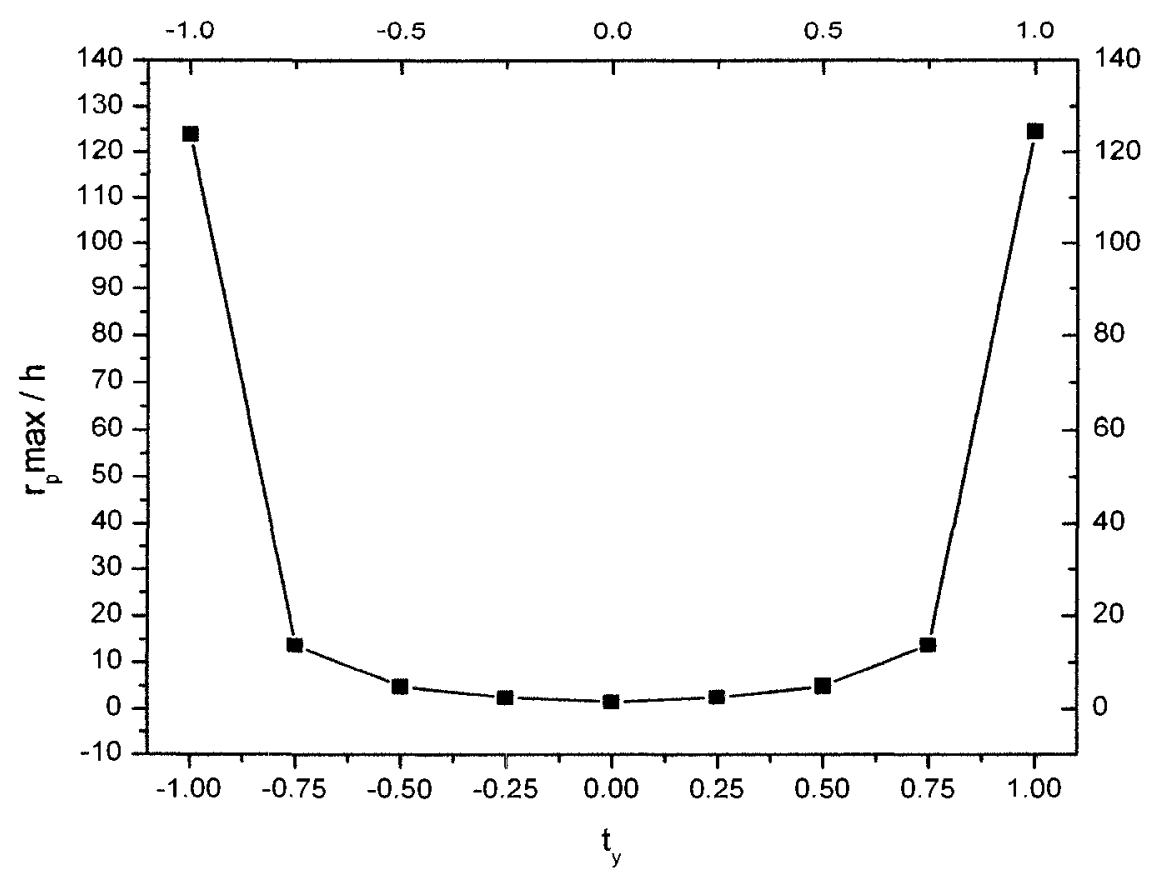

(i) $\phi=80^{\circ}$

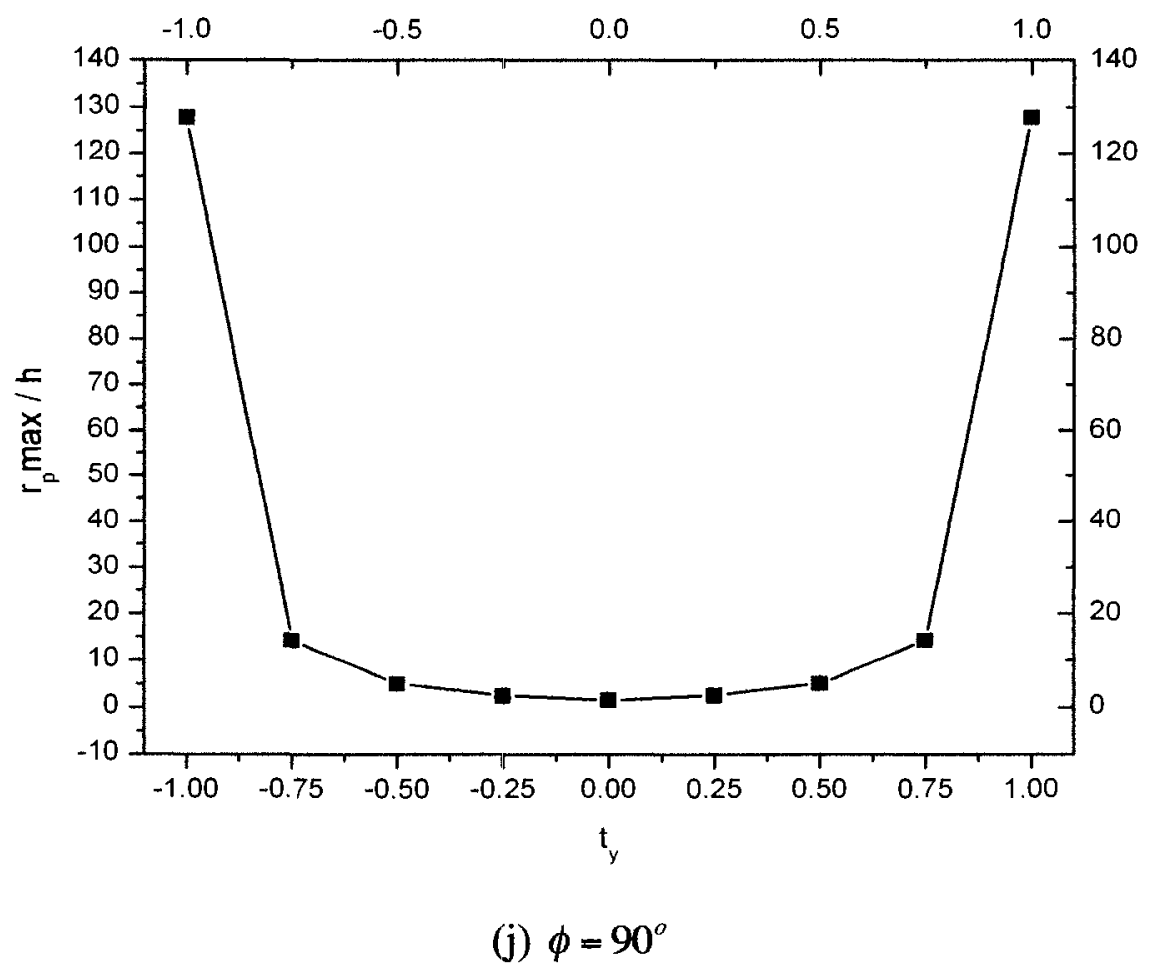

Figure 4.11: Graphs of maximum plastic zone size as a function of the normalized Tstress for various phase angles (i)-(j) 


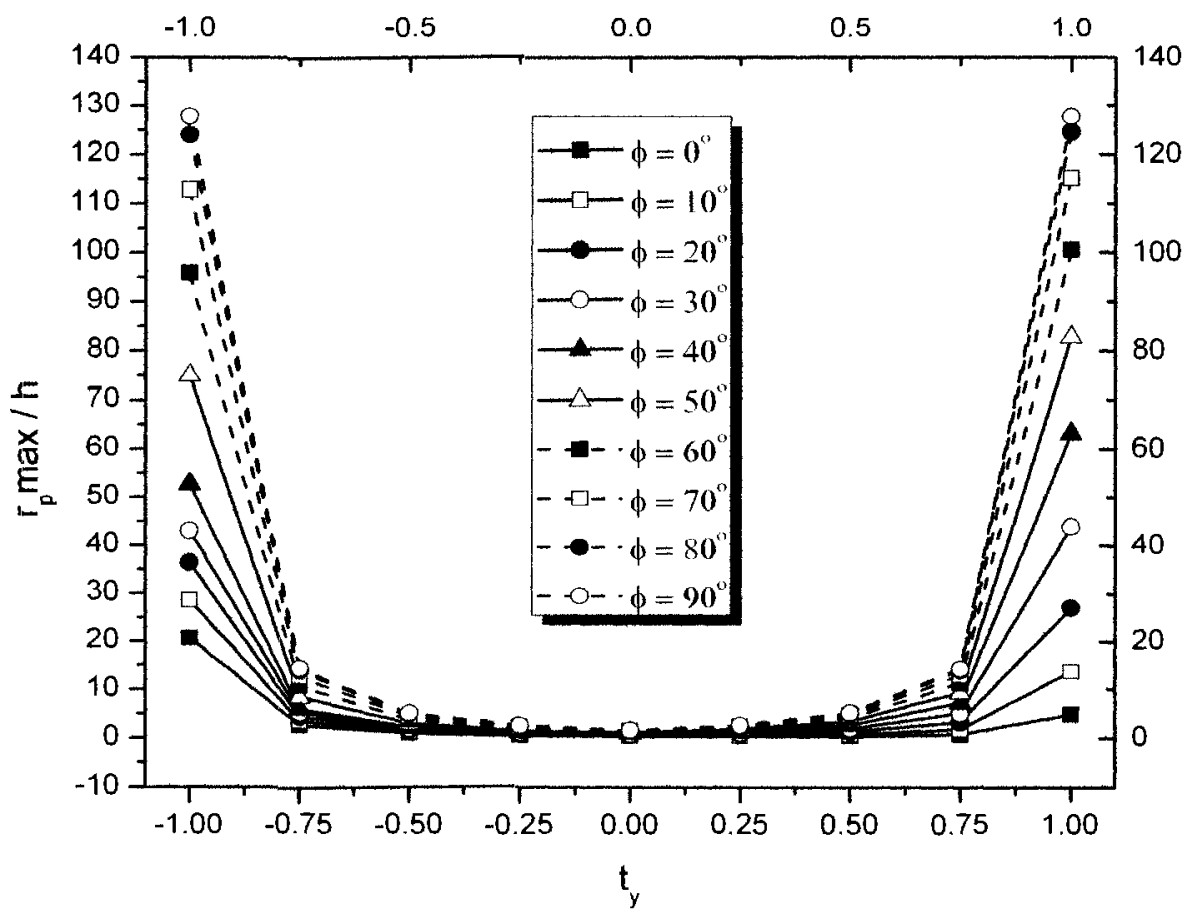

Figure 4.12: Combined graphs of maximum plastic zone size as a function of the normalized $\mathrm{T}$-stress $\left(-1 \leq t_{y} \leq 1\right)$ for various phase angles

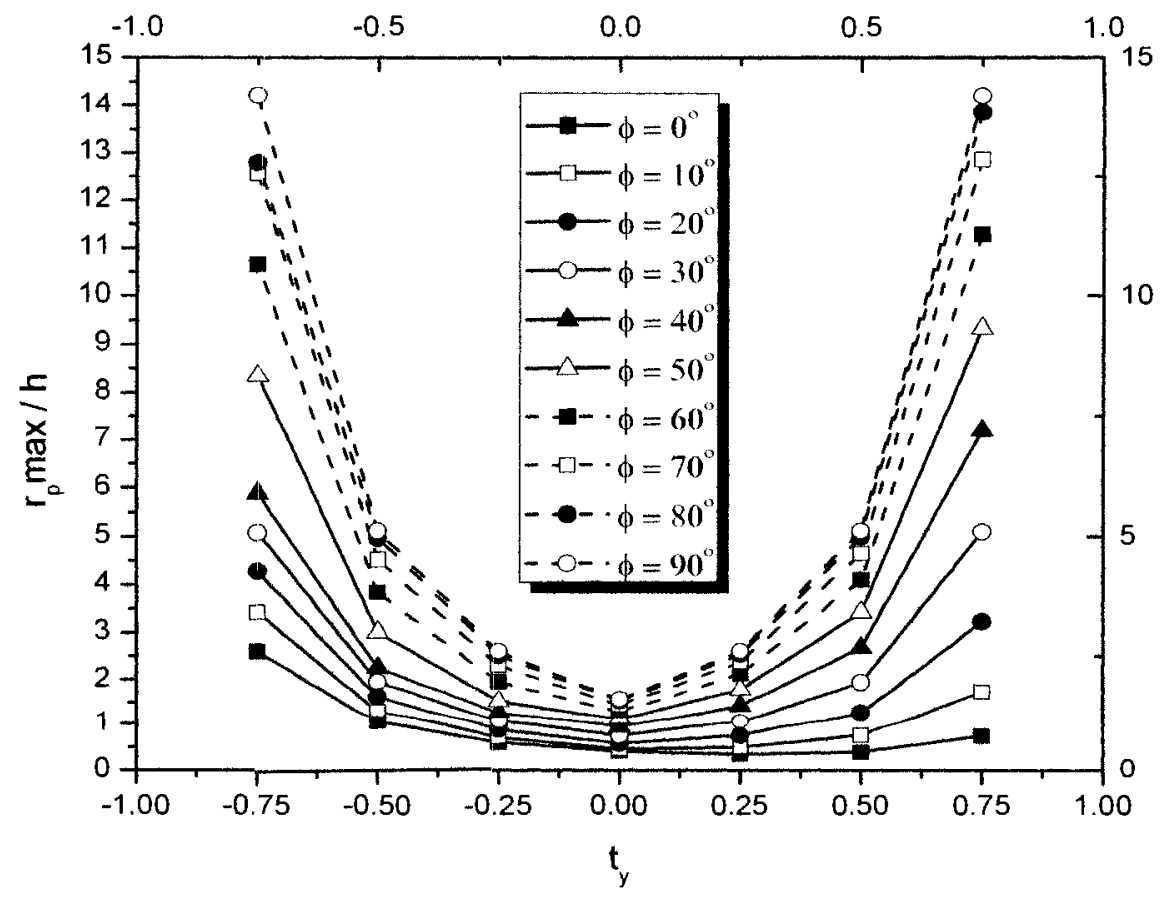

Figure 4.13: Combined graphs of maximum plastic zone size as a function of the normalized T-stress $\left(-0.75 \leq t_{y} \leq 0.75\right)$ for various phase angles 


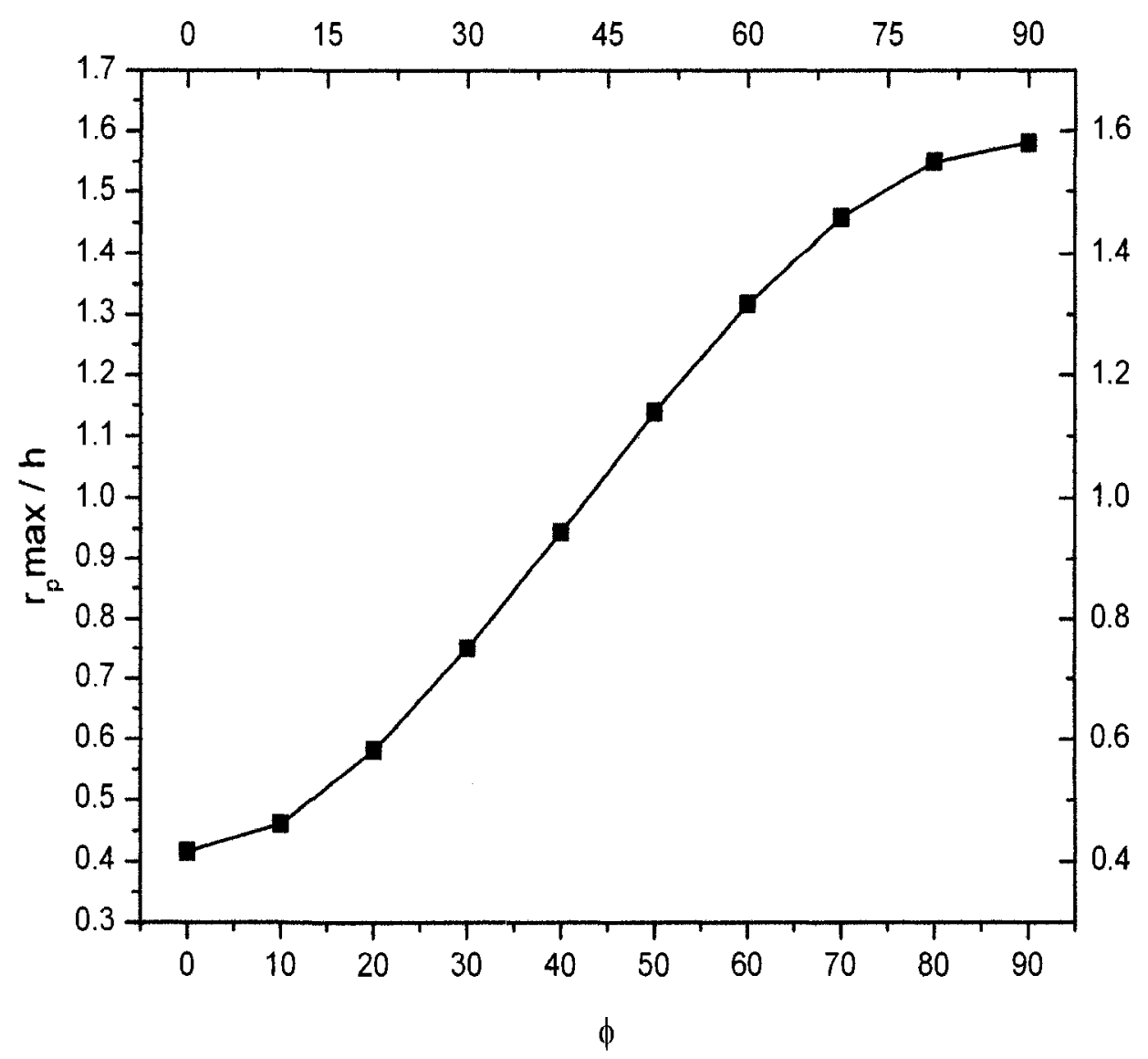

(a) $t_{y}=0$

Figure 4.14: Graphs of maximum plastic zone size as a function of phase angle for various normalized cyclic $\mathrm{T}$-stress values (a) 


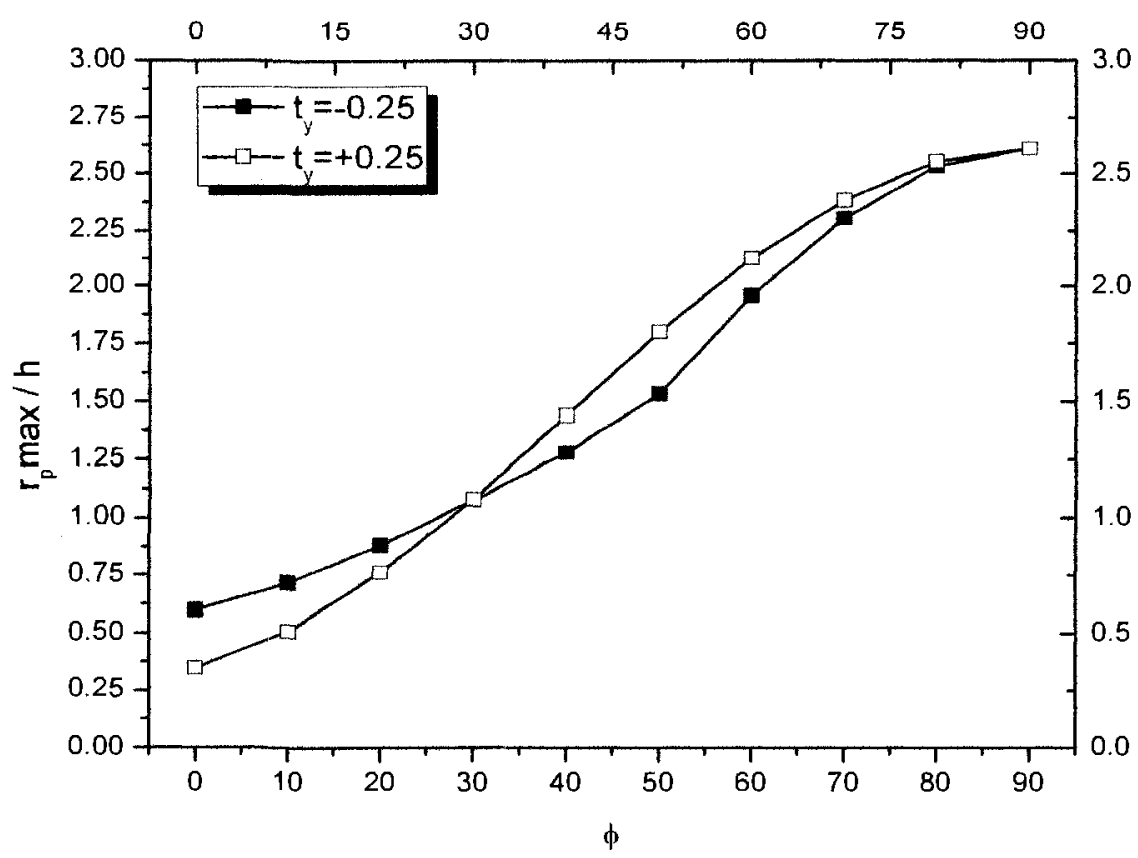

(b) $t_{y}= \pm 0.25$

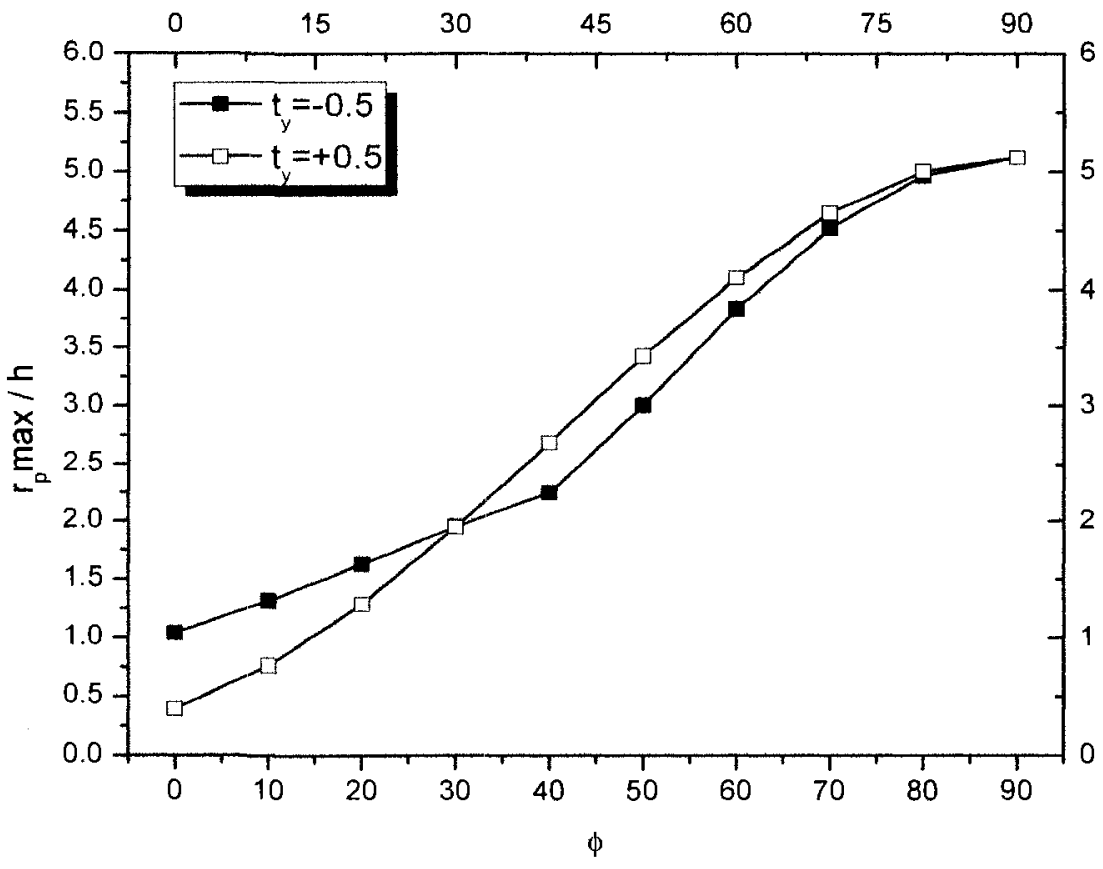

(c) $t_{y}= \pm 0.5$

Figure 4.14: Graphs of maximum plastic zone size as a function of phase angle for various normalized cyclic $\mathrm{T}$-stress values (b)-(c) 


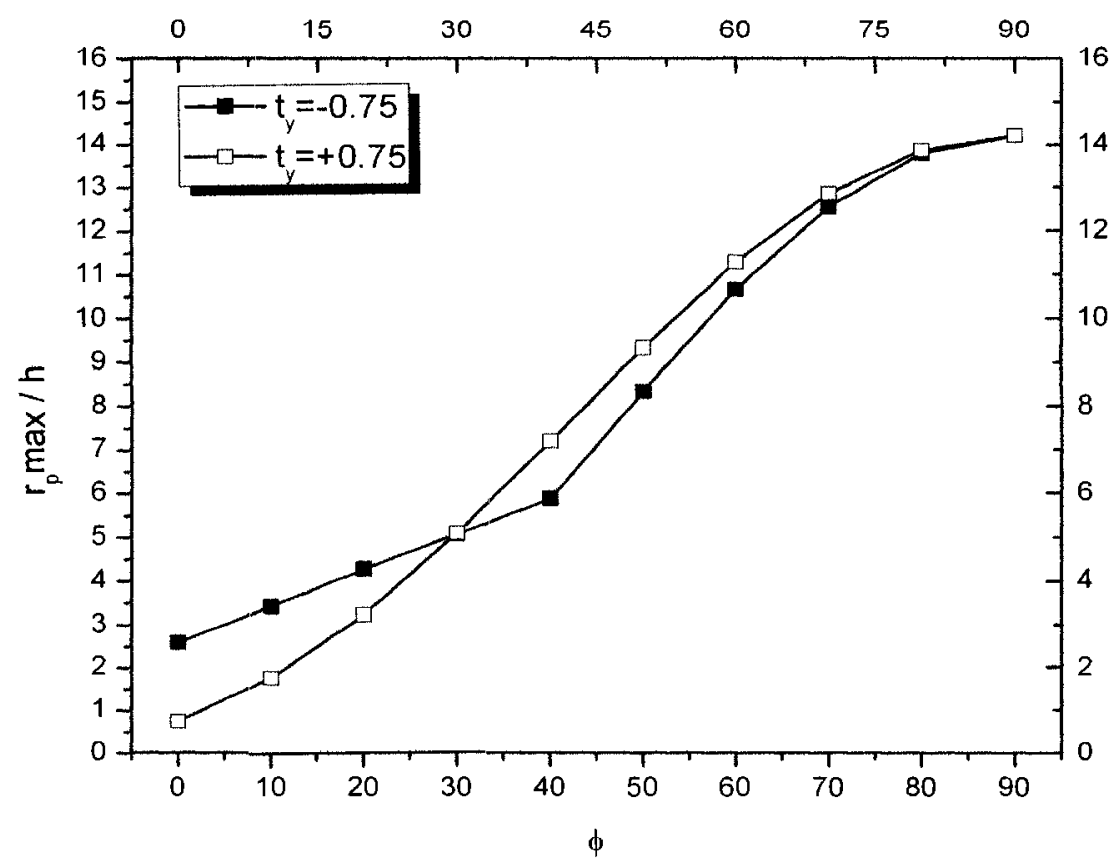

(d) $t_{y}= \pm 0.75$

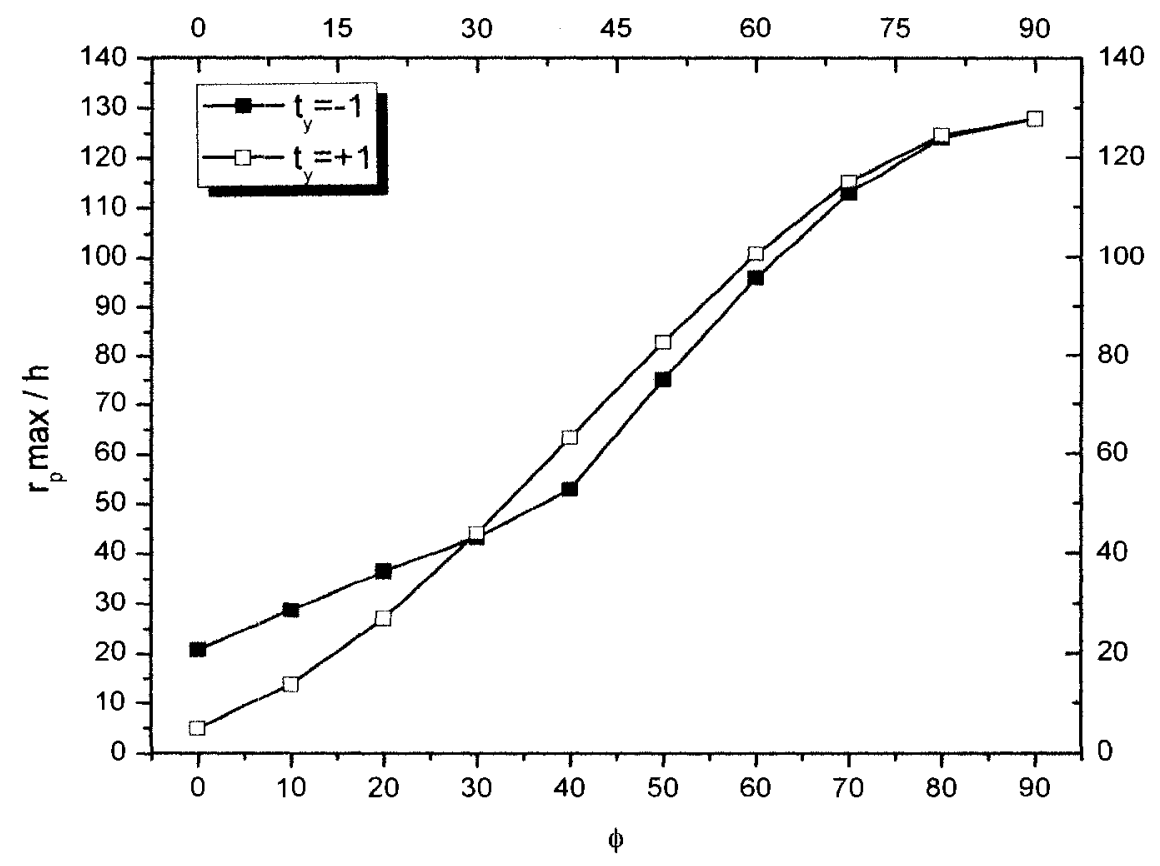

(e) $t_{y}= \pm 1$

Figure 4.14: Graphs of maximum plastic zone size as a function of phase angle for various normalized cyclic $T$-stress values (d)-(e) 


\section{Chapter 5}

\section{Crack-Tip Plastic Zone Analysis Under Cyclic Mixed-Mode Conditions}

In this chapter, the plastic zone expression developed in Chapter 4 for static loading conditions is adapted for cyclic loading. The cyclic plastic zone expression is then used to develop a mathematical model to better predict fatigue crack growth rates by taking into account the influence of not only the stress intensity factor (SIF) but of the Tstress as well.

This chapter also describes the application of the results developed in Chapter 3 to Gao et al's [1985] center cracked plate (CCP) specimen subjected to bi-axial mixed-mode loading for the purpose of demonstrating the significance of the T-stress when predicting fatigue crack growth rates.

\subsection{The Cyclic Plastic Zone and Crack Propagation Rates}

In this section, an expression to model the cyclic plastic zone is adapted from the static plastic zone expression developed in Chapter 4. The cyclic plastic zone model is then used to propose a crack propagation model that incorporates the effect of T-stress in addition to the SIF under mixed-mode loading.

\subsubsection{The Cyclic Plastic Zone}

The plastic zone under cyclic loading is developed from the plastic zone under static loading (Eqn 4.1) by replacing the SIF $K$, elastic T-stress $T$, and material yield stress $\sigma_{y}$ with their cyclic equivalents $\Delta K, \Delta T$, and $2 \sigma_{y}$ to yield: 
$r_{p-c y c i c}(\theta)=\frac{1}{2 \pi}\left(\frac{\Delta K}{2 \sigma_{y}}\right)^{2}\left[\frac{1}{\Delta c^{*}}\left\{-[b] \Delta t_{y}-\sqrt{[b]^{2} \Delta t_{y}^{2}-[a] \Delta c^{*}}\right\}\right]^{2}$

where,

The term $r_{p-c y c l i c}$ is the distance from the crack-tip where, for an applied stress field, the specimen begins to yield under cyclic loading.

The net cyclic SIF $\Delta K=\sqrt{\Delta K_{I}^{2}+\Delta K_{I I}^{2}}$

Phase angle $\phi=\tan ^{-1} \frac{\Delta K_{n}}{\Delta K_{I}}$

The Net cyclic SIF $K$ represents the net combined effect of the cyclic mode I SIF $\Delta K_{I}$ and mode II SIF $\Delta K_{I I}$ on the crack-tip, while $\phi$ represents the phase angle separating the cyclic mode I and mode II SIF's where $\tan \phi$ denotes the mode II to mode I cyclic SIF ratio. The boundary conditions of $\Delta K$ and $\phi$ are $\Delta K=\Delta K_{I}$ at $\phi=0^{\circ}$ for a pure mode I cyclic loading condition, and $\Delta K=\Delta K_{I}$ at $\phi=90^{\circ}$ for a pure mode II cyclic loading condition.

and

The normalized T-stress with respect to the yield stress $\Delta t_{y}=\frac{\Delta T}{2 \sigma_{y}}$

$\Delta c^{*}=[c] \Delta t_{y}^{2}-2$ 


$$
\begin{aligned}
{[a]=\left[\cos ^{2} \phi \cdot\left\{\frac{3}{2} \sin ^{2} \theta+V(\cos \theta+1)\right\}+\cos \phi \cdot \sin \phi \cdot\{\right.} & 2 \sin \theta \cdot(3 \cos \theta-V)\} \\
& \left.+\sin ^{2} \phi \cdot\left\{6-\frac{9}{2} \sin ^{2} \theta-V(\cos \theta-1)\right\}\right]
\end{aligned}
$$

$$
[b]=\left[\cos \phi \cdot\left\{\cos \frac{\theta}{2} \cdot\left(-3\left\{\cos ^{2} \frac{\theta}{2}-\cos ^{2} \theta\right\}+V\right)\right\}+\sin \phi \cdot\left\{\sin \frac{\theta}{2} \cdot\left(-3\left\{\cos ^{2} \frac{\theta}{2}+\cos ^{2} \theta\right\}-V\right)\right\}\right]
$$

$[c]=\left[\frac{1}{2}\{3+V\}\right]$

$$
V=(1-2 v)^{2}
$$

Note that $(r, \theta)$ are the polar coordinates centered at the crack-tip (Fig. 2.3), and $v$ is Poisson's ratio.

\subsubsection{Crack Propagation Rate Model}

To model crack propagation rates such that they take into account the effect of $\mathrm{T}$ stress on mixed mode I and mode II loading, the cyclic plastic zone size (PZS) expression developed in the previous sub-section (Eqn 5.1) is incorporated into the crack propagation relation developed by Wang \& Gao [2007] and introduced in Chapter 2 (Eqn 2.10).

Taking Equation 5.1 and incorporating it into the following crack propagation relation developed by Wang \& Gao [2007]:

$$
\frac{d a}{d N} \propto\left(r_{p-c y c l i c \max }\right)^{n / 2}
$$


where $d a / d N$ is the crack growth $(d a)$ per cycle $(d N), n$ is the experimentally determined material constant of the power law relationship, and $r_{p \text {-cyclic } \max }$ is the maximum cyclic PZS.

results in the following proportionality:

$\frac{d a}{d N} \propto\left(\frac{1}{2 \pi}\left(\frac{\Delta K}{2 \sigma_{y}}\right)^{2}\left[\frac{1}{\Delta c^{*}}\left\{-[b] \Delta t_{y}-\sqrt{[b]^{2} \Delta t_{y}^{2}-[a] \Delta c^{*}}\right\}\right]_{\max }^{2}\right)^{n / 2}$

Note that $[a],[b]$, and $c^{*}$ are trigonometric functions defined by Equations 4.6 and 5.5, and that the term $[a]$ is not the crack length $a$.

simplifying Equation 5.6 gives:

$\frac{d a}{d N} \propto\left((\Delta K)^{2}\left[\frac{1}{\Delta c^{*}}\left\{-[b] \Delta t_{y}-\sqrt{[b]^{2} \Delta t_{y}^{2}-[a] \Delta c^{*}}\right\}\right]_{\max }^{2}\right)^{n / 2}$

converting the proportionality in Equation 5.7 into an equality results in the following expression for the crack propagation rate:

$\frac{d a}{d N}=C\left(\Delta K \cdot f_{T}\right)^{n}$

where the term $f_{T}$, dubbed the cyclic T-factor by the author, is defined as:

$f_{T}=\left[\frac{1}{\Delta c^{*}}\left\{-[b] \Delta t_{y}-\sqrt{[b]^{2} \Delta t_{y}^{2}-[a] \Delta c^{*}}\right\}\right]_{\max }$

and $C$ is the experimentally determined material constant of the power law relationship. 
To better emulate the form of Paris \& Erdogan's original power law relationship, the net SIF and the cyclic T-factor of Equation 5.9 can be combined into a single term called the effective SIF as follows:

$$
\frac{d a}{d N}=C\left(\Delta K_{e f f}\right)^{n}
$$

where the effective SIF $\Delta K_{e f f}$ is defined as follows:

$$
\Delta K_{\text {eff }}=\left(\Delta K \cdot f_{T}\right)
$$

The crack propagation model described by Equation 5.10 can be used to predict the crack growth rate for any crack subjected to a general mixed mode I and mode II loading conditions influenced by T-stress, provided that the material constants of the power law relationship $C$ and $n$ are known.

\section{The Cyclic T-Factor}

The cyclic T-factor was calculated for a crack embedded in a linear elastic material with a Poisson's ratio of 0.3 subjected to mixed mode loading conditions with phase angles ranging from $\phi=0^{\circ}$ to $\phi=90^{\circ}$ and normalized cyclic T-stress $\Delta t_{y}$ values ranging from -1 to 1 . The results of the cyclic $T$-factor calculation are presented in Table 5.1 and, for convenience and ease of interpolation, in Figures 5.1a-j and 5.2 as a function of $\Delta t_{y}$, and Figures 5.3a-e as a function of $\phi$. Therefore, the cyclic T-factor can be interpolated from Figures 5.1 and/or 5.3 for a given loading condition and directly applied to Equation 5.10 to predict the crack growth rate. The symbols in Figures 5.1-5.3 represent the $\mathrm{T}$-factors calculated at those points using Equation 5.9, and the curves linking the data points are lines of fit used to show any trends present.

The variation of the cyclic T-factor as a function of the normalized cyclic T-stress can be observed in Figures 5.1a-j and 5.2. The figures show that the general trend of the cyclic T-factor versus normalized cyclic T-stress follows a squared-U-path. The cyclic T- 
factor drops sharply from $t_{y}=-1$ to $t_{y}=-0.75$ followed by a steady drop from $t_{y}=-0.75$ to $t_{y}=0$. The cyclic T-factor then shows a steady increase from $t_{y}=0$ to $t_{y}=0.75$ followed by a sharp increase from $t_{y}=0.75$ to $t_{y}=1$. From Figure 5.2, for $0^{\circ} \leq \phi \leq 20^{\circ}$ the cyclic T-factor is smaller for the positive T-stress values compared to the corresponding negative T-stress values with equal magnitude, while for $30^{\circ} \leq \phi \leq 70^{\circ}$ the reverse is true. For $\phi=90^{\circ}$, the curve is perfectly symmetric about the zero cyclic Tstress such that the cyclic T-factor is equal for cyclic T-stress values of equal magnitude but opposite sign. As $\phi$ increases from $0^{\circ}$ to $20^{\circ}$ the relative difference in the T-factor between the positive and corresponding negative cyclic T-stresses decreases. The cyclic $\mathrm{T}$-factor achieves almost perfect symmetry about the zero cyclic T-stress when $\phi=30^{\circ}$. As $\phi$ increases from $30^{\circ}$ to $50^{\circ}$ the relative difference in the cyclic $\mathrm{T}$-factor between the positive and corresponding negative cyclic T-stresses increases, and when $\phi$ increases from $60^{\circ}$ to $90^{\circ}$ the relative difference in the cyclic T-factor between the positive and corresponding negative cyclic $\mathrm{T}$-stresses again decreases until it reaches perfect equilibrium at $\phi=90^{\circ}$. The magnitude of the cyclic T-factor increases with $\phi$ at a given cyclic $\mathrm{T}$-stress. The largest and smallest values of the cyclic $\mathrm{T}$-factor are associated with $\phi=90^{\circ}$ and $\phi=0^{\circ}$ respectively.

The variation of the cyclic T-factor as a function of the phase angle is displayed in Figures 5.3a-e. The figures are such that the cyclic T-factors for cyclic T-stress values that are equal in magnitude but opposite in sign are grouped together in one graph. Note that curves with normalized cyclic T-stress values of equal magnitude but opposite signs are referred to as a "corresponding set". In general, the cyclic T-factor curves follow an $\mathrm{S}$-shape, with the cyclic $\mathrm{T}$-factor gradually increasing in value as the phase angle increases from $\phi=0^{\circ}$ to $\phi=90^{\circ}$. The cyclic T-factor value is larger for the negative Tstress compared to the corresponding positive T-stress when $0^{\circ} \leq \phi<30^{\circ}$, however for $30^{\circ}<\phi<90^{\circ}$ the trend reverses. The cyclic T-factor curves for all corresponding sets of T-stresses intersect at $\phi=30^{\circ}$ (where the switchover takes place) and then converge 
together at a single point when $\phi=90^{\circ}$. It is interesting to note that the above-mentioned characteristics are common to all corresponding sets.

\subsection{Application of the Cyclic Plastic Zone to a Test}

\section{Specimen}

It has been established in Chapter 3 that the PZS is dependent on the T-stress value, and it has also been determined that the rate of crack propagation can be directly related to the PZS. As such, it can be deduced that the rate of crack propagation is directly influenced by the T-stress. It has already been determined that the size of the cyclic plastic zone and therefore the fatigue crack propagation is dependent on the phase angle and the T-stress.

However, it has not yet been demonstrated if the T-stress inherent in an actual loaded part or specimen can be significant enough to have an effect on its fatigue crack propagation rate. To validate the significance of the T-stress in determining fatigue crack propagation rates accurately, the specimens tested by Gao et al [1985] to experimentally determine the relationship between the PZS and fatigue crack propagation rates was analyzed analytically using the information available in Chapters 2,3, and 4 of this thesis.

\subsubsection{The Test Specimen}

The experimental model used by Gao et al [1985] was a perfectly square center cracked plate (CCP) specimen (Fig. 5.4) of width $2 W$ and made of 316 stainless steel with a $2 \%$ offset yield stress of $246 \mathrm{MPa}$ and an ultimate tensile strength of $595 \mathrm{MPa}$. The CCP specimen was cyclically loaded bi-axially with a distributed load of $\sigma$ in the longitudinal direction and $\Lambda \sigma$ in the transverse direction, where $\Lambda$ is the bi-axial load ratio. The crack in the CCP specimen was of length $2 a$ oriented at an angle of $\alpha$ and $\beta$ with respect to the horizontal and vertical axes respectively. Gao et al [1985] conducted five tests on the CCP specimen; the details of which are described in Table 5.2. 


\subsubsection{Determining Stress Intensity Factors and T-Stress for the Test Specimen}

To determine the SIF's and T-stress values for the bi-axially loaded CCP specimen using data for a uniaxially loaded CCP specimen from Chapter 3, the principle of superposition was applied such that the SIF's and T-stress was first determined for the longitudinal and transverse loading conditions individually and then combined for a pure tensile case (Fig. 5.5a) as follows:

$$
\begin{aligned}
& \Delta K_{I}^{\text {Total }}=\left|\Delta K_{I}^{L}+\Delta K_{I}^{T}\right|=\sigma \sqrt{\pi a}\left\{\left|\Delta f_{I}^{L}+\Lambda \Delta f_{I}^{T}\right|\right\} \\
& \Delta K_{I I}^{\text {Total }}=\left|\Delta K_{I I}^{L}-\Delta K_{I I}^{T}\right|=\sigma \sqrt{\pi a}\left\{\left|\Delta f_{I I}^{L}-\Lambda \Delta f_{I I}^{T}\right|\right\} \\
& \Delta T^{\text {Total }}=\Delta T^{L}+\Delta T^{T}=\sigma\left\{\Delta t^{L}+\Lambda \Delta t^{T}\right\}
\end{aligned}
$$

where, $f_{I}$ and $f_{I I}$ are the normalized mode I and II SIF's respectively, and $t$ is the normalized T-stress with respect to the applied load. The superscripts: Total, L, and T, refer to the stress states in the Total (longitudinal and transverse), Longitudinal, and Transverse loading conditions respectively.

Note that the SIF and T-stress values cannot be directly obtained for the transversely loaded component when applying the principle of superposition (Fig 5.5a). An equivalent model to that of the transversely loaded component, as shown in Figure $5.5 \mathrm{~b}$, was first determined such that the equivalent model could be used to directly obtain SIF and T-stress data for the transversely loaded component from the data available in Chapter 3.

The values of the normalized SIF's and T-stress were obtained from Figures 3.103.12 of Chapter 3. The SIF and T-stress values were obtained directly from the figures for 
$\alpha=\beta=45^{\circ}$, and interpolated for $\alpha=67.5^{\circ}$ (from $\alpha=60^{\circ}$ and $\alpha=75^{\circ}$ ) and $\beta=22.5^{\circ}$ (from $\beta=15^{\circ}$ and $\beta=30^{\circ}$ ).

The results obtained from data in Chapter 3 for the tests conducted by Gao et al [1985] on the bi-axially loaded CCP specimen are displayed in Table 5.3, while comparisons of analytical values against those obtained experimentally by Gao et al [1985] are shown in Table 5.4 The maximum difference between the analytical values and that of Gao et al's [1985] values was found to be $8.8 \%$ while the majority of the values differed by less than 5\% (further validating the results obtained for the SIF's and T-stress in Chapter 3). From Table 5.3, it can be seen that Test 1 is a case of pure shear with a phase angle of $\phi=90^{\circ}$, that is, there exists only a mode II SIF component and no T-stress. Tests 2 and 5 have a comparably small negative T-stress value of the order 1 with phase angles of $\phi=71.2^{\circ}$ and $\phi=39.1^{\circ}$ respectively, while Tests 3 and 4 have a comparably large positive T-stress value of the order 10 with phase angles of $\phi=87.5^{\circ}$ and $\phi=53.5^{\circ}$ respectively.

\subsubsection{The Cyclic Plastic Zone for the Test Specimen}

The results provided in Table 5.3 for Gao et al's [1985] bi-axially loaded CCP specimen are used to calculate the normalized cyclic PZS $r_{p-c y c l i c} / \Delta h$ by applying the following expression developed from Equation 5.1:

$\frac{r_{p-c y c l i c}(\theta)}{\Delta h}=\frac{1}{2}\left[\frac{1}{\Delta c^{*}}\left\{-[b] \Delta t_{y}-\sqrt{[b]^{2} \Delta t_{y}^{2}-[a] \Delta c^{*}}\right\}\right]^{2}$

where,

$$
\Delta h=\frac{1}{\pi}\left(\frac{\Delta K}{2 \sigma_{y}}\right)^{2}
$$


The normalized cyclic plastic zones were calculated twice for each test and the maximum value identified; the first calculation neglected the inherent $T$-stress while the second calculation took the T-stress into account. The comparisons of the normalized cyclic plastic zones with and without accounting for T-stress are available in Tables 5.5 and 5.6 respectively with the corresponding maps displayed in Figures 5.6a-e.

Note that in Figures 5.6a-e the cracks emanate from the left-hand-side of the graphs with the crack-tips located at the origin in the center of the graph. The cyclic plastic zones are mapped with the Cartesian coordinate system using the polar to Cartesian conversions shown as follows:

$$
\begin{aligned}
& x_{p-c y c l i c}=r_{p-c y c l i c} \cdot \cos \theta \\
& y_{p-c y c l i c}=r_{p-c y c l i c} \cdot \sin \theta
\end{aligned}
$$

The normalized cyclic plastic zones for the purpose of research were calculated at one-degree intervals, however due to the amount of data involved, Tables 5.5 and 5.6 display the results at fifteen-degree intervals. Since Test 1 has zero T-stress, the cyclic plastic zone map derived from SIF's alone is not affected by T-stress and remains unchanged (Fig. 5.6a). From the plastic zone maps of Tests 2 and 5 (Fig. 5.6b and 5.6e), it can be seen that for small magnitudes of T-stress there is no perceptible variation in the normalized cyclic plastic zone when compared to the case without T-stress; the two plastic zones virtually overlap. From the plastic zone maps of Tests 3 and 4 (Fig. 5.6c and 5.6d), there was a significant variation in the maps of the plastic zones that took the Tstress in account and those that did not; the most notable difference is the discontinuity across the crack-front and the increased size of the plastic zone that took the T-stress into account. Overall, the observations of Tests 1-5, including the size, shape, and orientation of the plastic zone maps (Fig. 5.6a-e), corroborate the results detailed in Chapter 4.

Table 5.7 and Figure 5.7 provide comparisons of the maximum cyclic PZS calculated with and without considering the T-stress for all five of Gao et al's [1985] 
tests. Since Test 1 has zero T-stress, the maximum PZS calculated using SIF's alone is not affected by the T-stress. From Tests 2 and 5, the difference in the value of the maximum cyclic PZS with respect to the value that neglects the T-stress is less than $0.3 \%$, while the difference in the maximum PZS for Test 3 and 4 is over $15 \%$. Having a difference in cyclic PZS of $15 \%$ is a clear indicator that the SIF alone cannot accurately predict fatigue crack growth rates and as such, the T-stress must be considered for accurate results.

\subsection{Discussion}

From the analysis of Gao et al's [1985] bi-axially loaded model, it is observed that the T-stress for different loading scenarios are not always significant enough to affect the size of the specimen's plastic zone. However, in those cases where the T-stress was found to be significant, its influence on the PZS is considerable enough to necessitate its inclusion in the analysis and prediction of fatigue crack propagation rates. As such, to ensure improved accuracy when predicting crack growth rates, the T-stress effect must be considered.

The fatigue crack propagation rate is observed to be affected directly by the cyclic T-factor such that, for a given net SIF, the rate of crack growth increased with the magnitude of the cyclic $\mathrm{T}$-factor. The cyclic $\mathrm{T}$-factor quantifies the $\mathrm{T}$-stress effect by amplifying the net SIF to obtain the effective SIF. The effective SIF can be defined as the SIF, which by itself, produces the same crack propagation rate as that of the combined net SIF and T-stress effect.

The cyclic $\mathrm{T}$-factor was found to increase with the magnitude of the T-stress irrespective of its sign, and it was also found to increase with the phase angle. Therefore, cracks with either large values of T-stress and/or a dominant mode II SIF component had a larger cyclic T-factor and therefore resulted in faster growing fatigue cracks, while on the other hand, cracks with a zero or close to zero T-stress value and/or a dominant mode I SIF component had a smaller cyclic T-factor which resulted in a slower crack growth. As such, to maximize the fatigue life of a given part, the cyclic T-factor needs to be 
minimized by reducing the T-stress and/or the mode II to mode I SIF ratio to as close to zero as possible.

It was observed that the cyclic T-factor for a phase angle less than $30^{\circ}$ was larger for a negative $T$-stress than for a positive $T$-stress of equal magnitude, while for a phase angle greater than $30^{\circ}$ but less than $90^{\circ}$, the cyclic T-factor was observed to be larger for the positive $\mathrm{T}$-stress than for the corresponding negative T-stress. The cyclic T-factor for the phase angles of $30^{\circ}$ and $90^{\circ}$ were observed to be approximately equal. Therefore, the rate of crack growth for negative T-stress values as compared to that of positive T-stress values is:

a) Larger only if the mode II to mode I SIF ratio of less than $\tan 30^{\circ}$.

b) Smaller only if the mode II to mode I SIF ratio is between $\tan 30^{\circ}$ and $\tan 90^{\circ}$.

c) Equal only when the mode II to mode I SIF ratio is equal to $\tan 30^{\circ}$ or $\tan 90^{\circ}$.

The observation described in point a) above coincides with the observations of Hutar et al [2004] and Seitl \& Knesl [2008] for a mode I $\left(\phi=0^{\circ}\right)$ loading condition influenced by Tstress.

Note that above, the mode II to mode I SIF ratio is discussed with respect to its effect on the cyclic $\mathrm{T}$-factor and not its direct effect on the crack propagation rate.

\subsection{Conclusion}

The static plastic zone expression developed in Chapter 4 was adapted for cyclic loading conditions. The cyclic plastic zone expression was used to develop an expression to better predict crack propagation rates by taking into consideration the effect of T-stress under mixed-mode loading. 
Through the analysis of a bi-axially loaded center cracked plate specimen, it has been demonstrated that the T-stress effect is significant enough to warrant its implementation in the prediction of crack growth rates. By incorporating the $\mathrm{T}$-stress effect, a more accurate prediction of crack growth and therefore fatigue life can be obtained. 
Table 5.1: Table of T-factors for varying mode II to mode I SIF ratios and normalized T-stress values

\begin{tabular}{cccccccccc}
\hline & \multicolumn{7}{c}{ Cyclic Normalized $\tilde{T}$-stress, $\Delta \mathrm{t}_{\mathrm{y}}$} \\
\cline { 2 - 8 } & -1 & -0.75 & -0.5 & -0.25 & 0 & 0.25 & 0.5 & 0.75 & 1 \\
\hline $0^{\circ}$ & 6.4304 & 2.2760 & 1.4451 & 1.0942 & 0.9123 & 0.8356 & 0.8948 & 1.2200 & 3.1217 \\
$10^{\circ}$ & 7.5616 & 2.6122 & 1.6201 & 1.1945 & 0.9599 & 1.0053 & 1.2304 & 1.8687 & 5.2317 \\
$20^{\circ}$ & 8.5257 & 2.9227 & 1.8034 & 1.3273 & 1.0779 & 1.2329 & 1.6039 & 2.5418 \\
$30^{\circ}$ & 9.2862 & 3.1875 & 1.9760 & 1.4692 & 1.2253 & 1.7150 & 1.9741 & 3.1948 & 9.3754 \\
$40^{\circ}$ & 10.280 & 3.4279 & 2.1221 & 1.6013 & 1.3739 & 1.6973 & 2.3175 & 3.7962 & 11.241 \\
$50^{\circ}$ & 12.248 & 4.0847 & 2.4511 & 1.7509 & 1.5098 & 1.8976 & 2.6189 & 4.3215 & 12.865 \\
$60^{\circ}$ & 18.846 & 4.6176 & 2.7709 & 1.9793 & 1.6230 & 2.0623 & 2.8657 & 4.7514 & 14.194 \\
$70^{\circ}$ & 15.029 & 5.0116 & 3.0070 & 2.1478 & 1.7076 & 2.1848 & 3.0493 & 5.0710 & 15.183 \\
$80^{\circ}$ & 15.749 & 5.2519 & 3.1514 & 2.2510 & 1.7601 & 2.2603 & 3.1619 & 5.2668 & 15.788 \\
\hline 9 & 15.989 & 5.3321 & 3.1996 & 2.2855 & 1.7776 & 2.2855 & 3.1996 & 5.3321 & 15.989 \\
\hline
\end{tabular}

Note: $\mathrm{K}_{\mathrm{II}} / \mathrm{K}_{\mathrm{I}}=\tan \phi$ 
Table 5.2: Test details for Gao et al's [1985] bi-axially loaded CCP specimen

\begin{tabular}{cccccc}
\hline Test & $a(\mathrm{~mm})$ & $\begin{array}{c}a / W \\
(W=4 / \mathrm{mm})\end{array}$ & $\alpha$ & $\Delta \sigma(\mathrm{MPa})$ & $\Lambda$ \\
\hline 1 & 5.01 & 0.1222 & $45^{\circ}$ & 48.89 & -1 \\
2 & 7.52 & 0.1834 & $45^{\circ}$ & 48.89 & -0.5 \\
3 & 8.26 & 0.2015 & $67.5^{\circ}$ & 67.39 & -0.1716 \\
4 & 8.20 & 0.2 & $67.5^{\circ}$ & 74.82 & 0.082 \\
5 & 4.67 & 0.1139 & $45^{\circ}$ & 90.85 & 0.1 \\
\hline
\end{tabular}

Table 5.3: SIF's and T-stress results for Gao et al's [1985] specimen

\begin{tabular}{|c|c|c|c|c|c|}
\hline Test & $\Delta K_{I}\left(M N / \sqrt{m^{3}}\right)$ & $\Delta K_{I I}\left(M N / \sqrt{m^{3}}\right)$ & $\Delta K\left(M N / \sqrt{m^{3}}\right)$ & $\phi$ & $\Delta T(M P a)$ \\
\hline 1 & 0 & 6.17 & 6.17 & $0^{\circ}$ & 0 \\
\hline 2 & 1.94 & 5.70 & 6.02 & $71.2^{\circ}$ & -0.58 \\
\hline 3 & 0.19 & 4.43 & 4.43 & $87.5^{\circ}$ & 53.56 \\
\hline 4 & 2.85 & 3.85 & 4.79 & $53.5^{\circ}$ & 45.97 \\
\hline 5 & 6.12 & 4.98 & 7.89 & $39.1^{\circ}$ & -1.01 \\
\hline
\end{tabular}


Table 5.4: Comparison of SIF and T-stress results with that of Gao et al [1985]

\begin{tabular}{|c|c|c|c|c|c|c|c|c|c|}
\hline \multirow{2}{*}{ Test } & \multicolumn{3}{|c|}{$\Delta \mathrm{K}_{\mathrm{I}}\left(\mathrm{MN} / \mathrm{m}^{-3 / 2}\right)$} & \multicolumn{3}{|c|}{$\Delta \mathrm{K}_{\mathrm{II}}\left(\mathrm{MN} / \mathrm{m}^{-3 / 2}\right)$} & \multicolumn{3}{|c|}{$\Delta \mathrm{T}(\mathrm{MPa})$} \\
\hline & Analytical & $\begin{array}{c}\text { Gao et al } \\
{[1985]}\end{array}$ & $\begin{array}{c}\% \\
\text { Difference }\end{array}$ & Analytical & $\begin{array}{c}\text { Gao et al } \\
{[1985]}\end{array}$ & $\begin{array}{c}\% \\
\text { Difference }\end{array}$ & Analytical & $\begin{array}{c}\text { Gao et al } \\
{[1985]}\end{array}$ & $\begin{array}{c}\% \\
\text { Difference }\end{array}$ \\
\hline 1 & 0 & 0 & 0 & 6.17 & 6.18 & 0.2 & 0 & 0 & 0 \\
\hline 2 & 1.94 & 1.88 & 3.2 & 5.70 & 5.63 & 1.2 & -0.58 & 0 & N/A \\
\hline 3 & 0.19 & 0 & N/A & 4.43 & 4.54 & 2.4 & 53.56 & 55.83 & 4.1 \\
\hline 4 & 2.85 & 2.62 & 8.8 & 3.85 & 3.93 & 2.0 & 45.97 & 48.57 & 5.4 \\
\hline 5 & 6.12 & 6.08 & 0.7 & 4.98 & 4.98 & 0 & -1.01 & 0 & N/A \\
\hline
\end{tabular}


Table 5.5: Normalized cyclic plastic zone size results neglecting T-stress for Tests 1-5 of Gao et al [1985]

\begin{tabular}{cccccc}
\hline & Test 1 & Test 2 & Test 3 & Test 4 & Test 5 \\
\cline { 2 - 6 }$\theta$ & $\Delta K=6.17$, & $\Delta K=6.02$, & $\Delta K=4.43$, & $\Delta K=4.79$, & $\Delta K=7.89$, \\
& $\phi=0^{\circ}$ & $\phi=71.2^{\circ}$ & $\phi=87.5^{\circ}$ & $\phi=53.5^{\circ}$ & $\phi=39.1^{\circ}$ \\
\hline$-180^{\circ}$ & 0 & 1.4157 & 1.5768 & 1.0223 & 0.6288 \\
$-165^{\circ}$ & 0.0265 & 1.4704 & 1.5173 & 1.1711 & 0.8077 \\
$-150^{\circ}$ & 0.0991 & 1.3795 & 1.3206 & 1.2014 & 0.9116 \\
$-135^{\circ}$ & 0.1992 & 1.1679 & 1.0388 & 1.1066 & 0.9146 \\
$-120^{\circ}$ & 0.3012 & 0.8923 & 0.7462 & 0.9133 & 0.8179 \\
$-105^{\circ}$ & 0.3795 & 0.6261 & 0.5198 & 0.6741 & 0.6489 \\
$-90^{\circ}$ & 0.4150 & 0.4394 & 0.4184 & 0.4532 & 0.4541 \\
$-75^{\circ}$ & 0.4002 & 0.3809 & 0.4670 & 0.3095 & 0.2864 \\
$-60^{\circ}$ & 0.3413 & 0.4644 & 0.6506 & 0.2806 & 0.1908 \\
$-45^{\circ}$ & 0.2558 & 0.6656 & 0.9180 & 0.3730 & 0.1927 \\
$-30^{\circ}$ & 0.1684 & 0.9284 & 1.1958 & 0.5601 & 0.2905 \\
$-15^{\circ}$ & 0.1038 & 1.1804 & 1.4081 & 0.7899 & 0.4568 \\
$0^{\circ}$ & 0.0800 & 1.3523 & 1.4971 & 0.9987 & 0.6452 \\
$15^{\circ}$ & 0.1038 & 1.3965 & 1.4385 & 1.1286 & 0.8033 \\
$30^{\circ}$ & 0.1684 & 1.3002 & 1.2481 & 1.1428 & 0.8866 \\
$45^{\circ}$ & 0.2558 & 1.0886 & 0.9776 & 1.0359 & 0.8709 \\
$60^{\circ}$ & 0.3413 & 0.8184 & 0.7004 & 0.8353 & 0.7583 \\
$75^{\circ}$ & 0.4002 & 0.5625 & 0.4926 & 0.5942 & 0.5776 \\
$90^{\circ}$ & 0.4150 & 0.3906 & 0.4115 & 0.3768 & 0.3759 \\
$105^{\circ}$ & 0.3795 & 0.3502 & 0.4810 & 0.2417 & 0.2066 \\
$120^{\circ}$ & 0.3012 & 0.4539 & 0.6845 & 0.2262 & 0.1149 \\
$135^{\circ}$ & 0.1992 & 0.6759 & 0.9695 & 0.3355 & 0.1258 \\
$150^{\circ}$ & 0.0991 & 0.9589 & 1.2614 & 0.5422 & 0.2373 \\
$165^{\circ}$ & 0.0265 & 1.2290 & 1.4833 & 0.7928 & 0.4207 \\
$180^{\circ}$ & 0 & 1.4157 & 1.5768 & 1.0223 & 0.6288 \\
\hline
\end{tabular}

Note: Units for $\Delta K$ are $M N / \sqrt{m^{3}}$. 


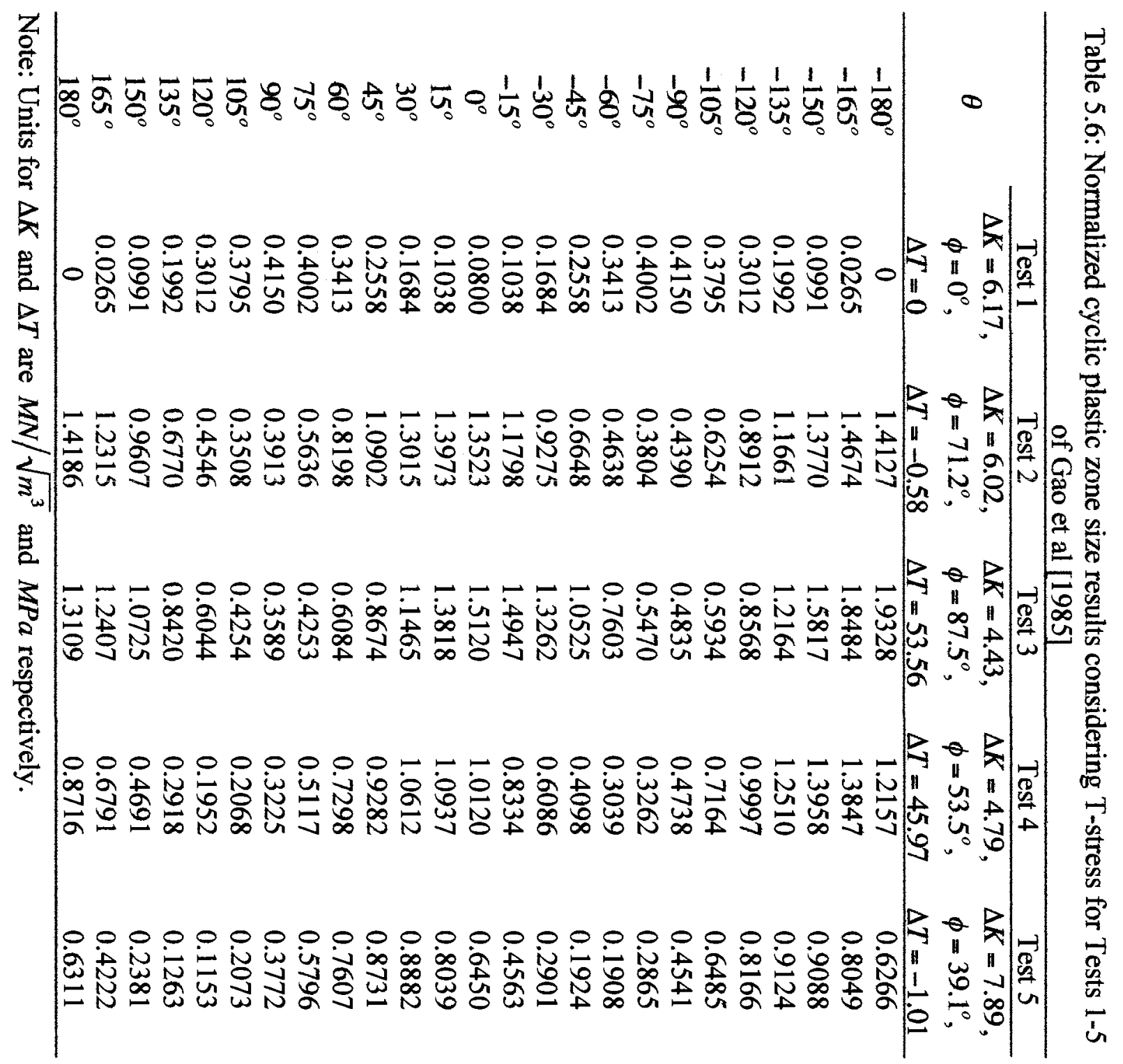


Table 5.7: Maximum normalized plastic zone results for Tests 1-5 of Gao et al [1985]

\begin{tabular}{cccc}
\hline \multirow{2}{*}{ Test } & \multicolumn{2}{c}{ Maximum Normalized Cyclic Plastic } & \multirow{2}{*}{ Zone Size Results } \\
\cline { 2 - 3 } & Neglecting T-Stress & $\begin{array}{c}\text { Considering T- } \\
\text { Stress }\end{array}$ & \\
\hline 1 & 0.4161 & 0.4161 & 0 \\
2 & 1.4716 & 1.4686 & 0.20 \\
3 & 1.5778 & 1.9338 & 22.56 \\
4 & 1.2059 & 1.4114 & 17.04 \\
5 & 0.9264 & 0.9238 & 0.28 \\
\hline
\end{tabular}




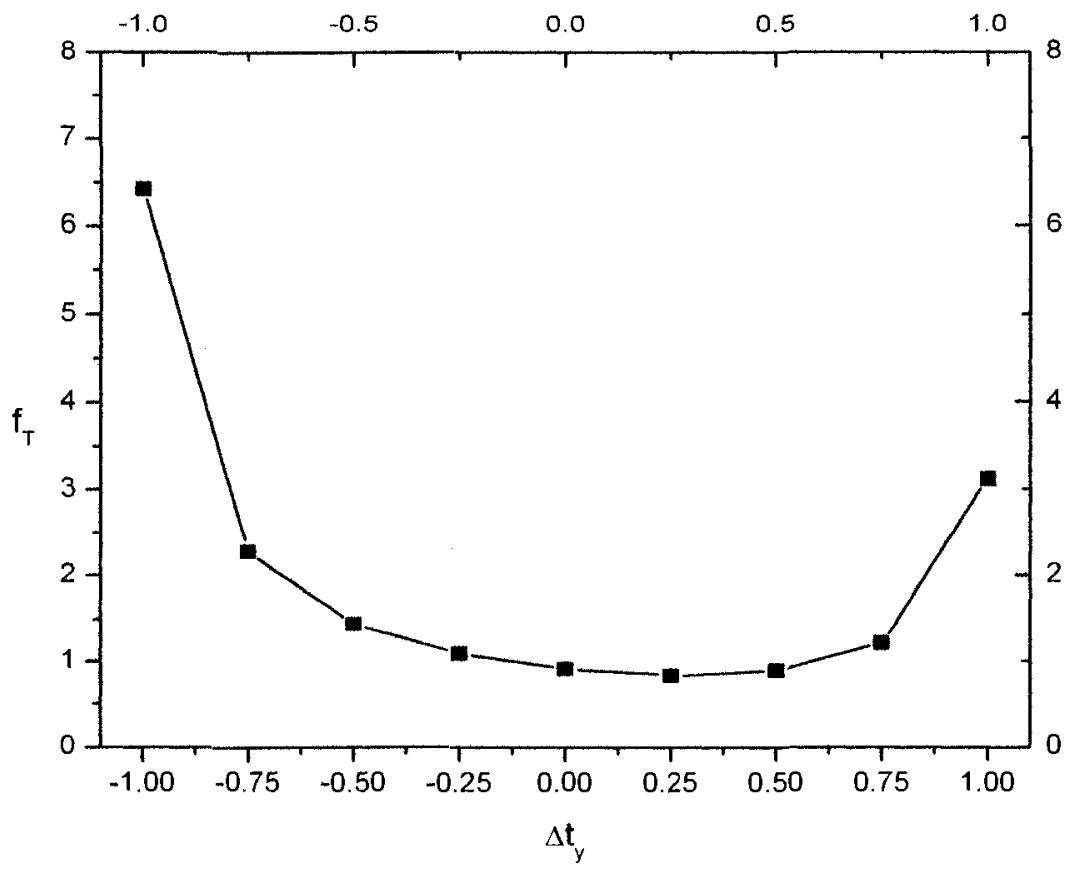

(a) $\phi=0^{\circ}$

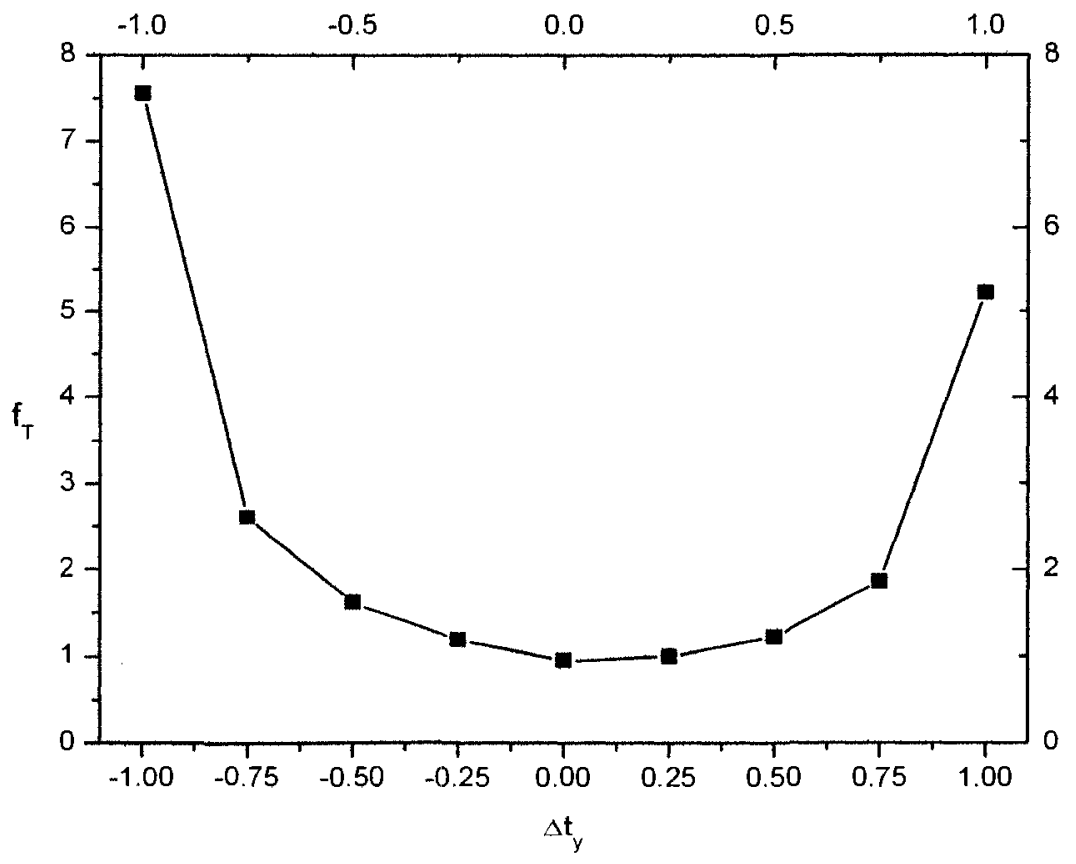

(b) $\phi=10^{\circ}$

Figure 5.1: Graphs of T-factor as a function of the normalized cyclic T-stress for various phase angles (a)-(b) 


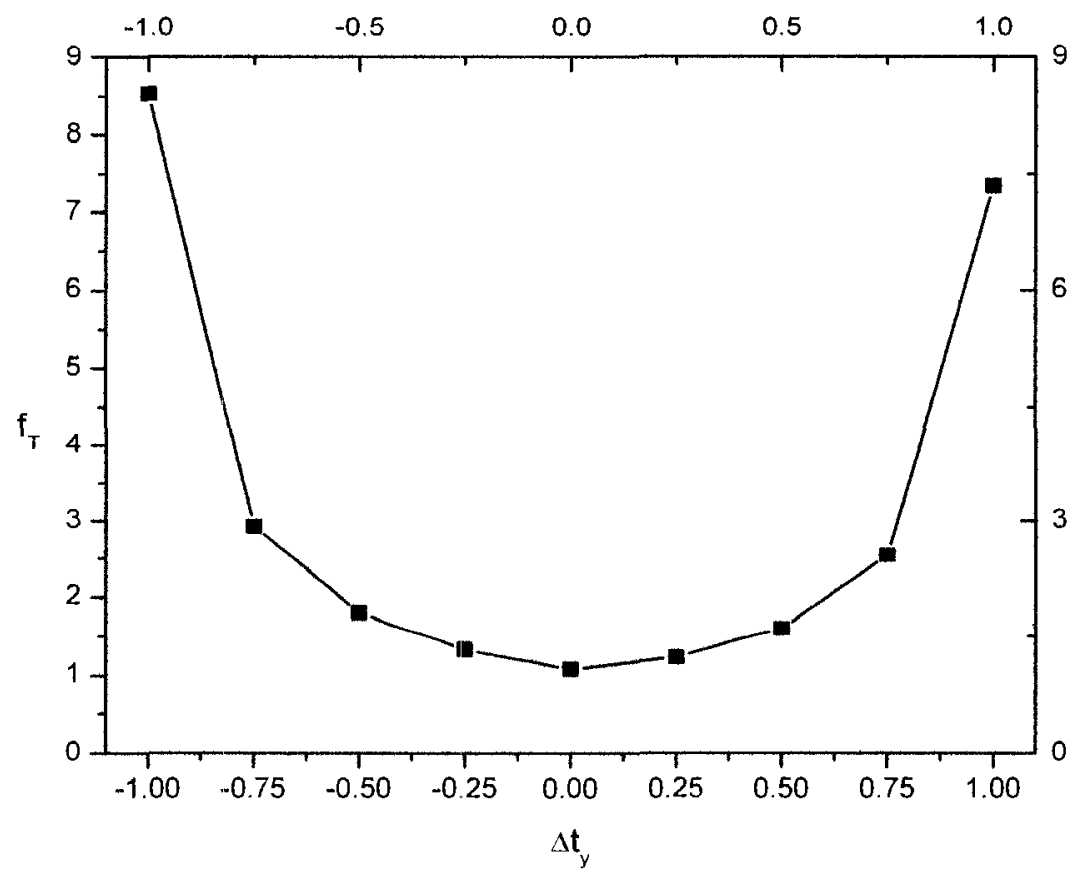

(c) $\phi=20^{\circ}$

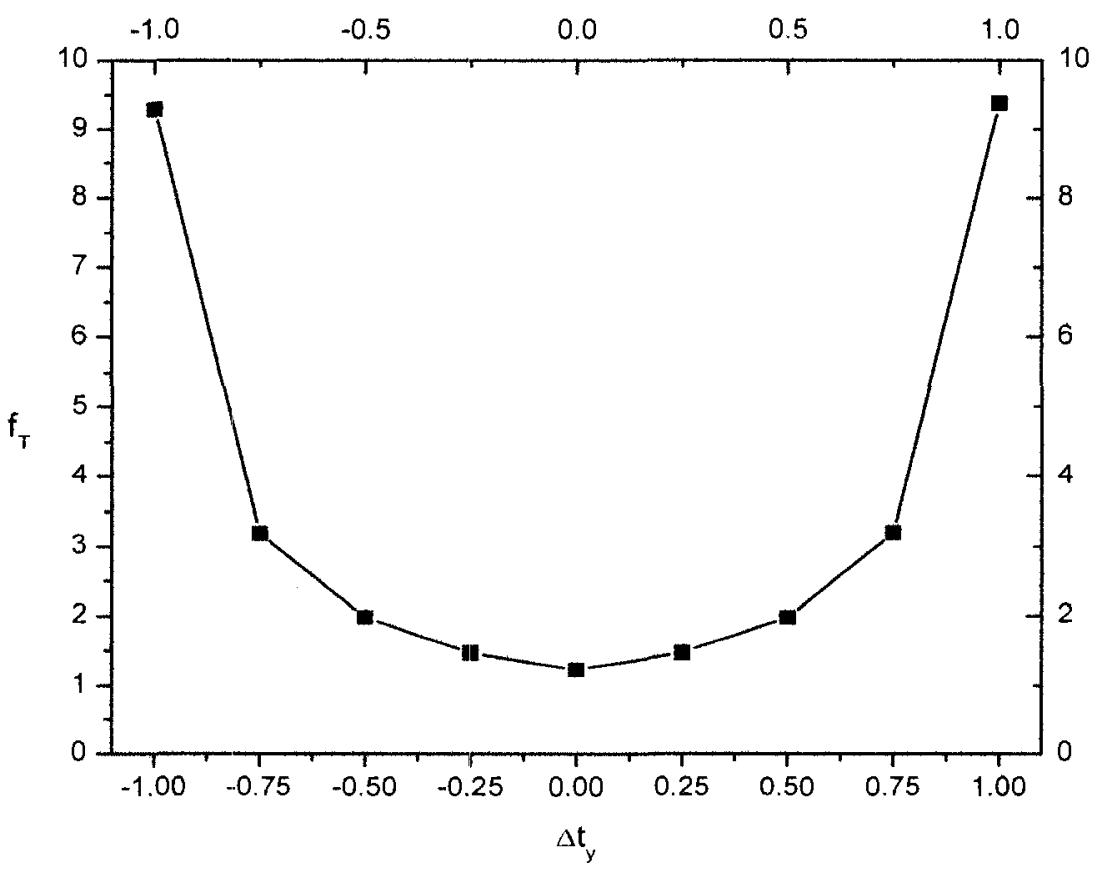

(d) $\phi=30^{\circ}$

Figure 5.1: Graphs of T-factor as a function of the normalized cyclic T-stress for various phase angles (c)-(d) 


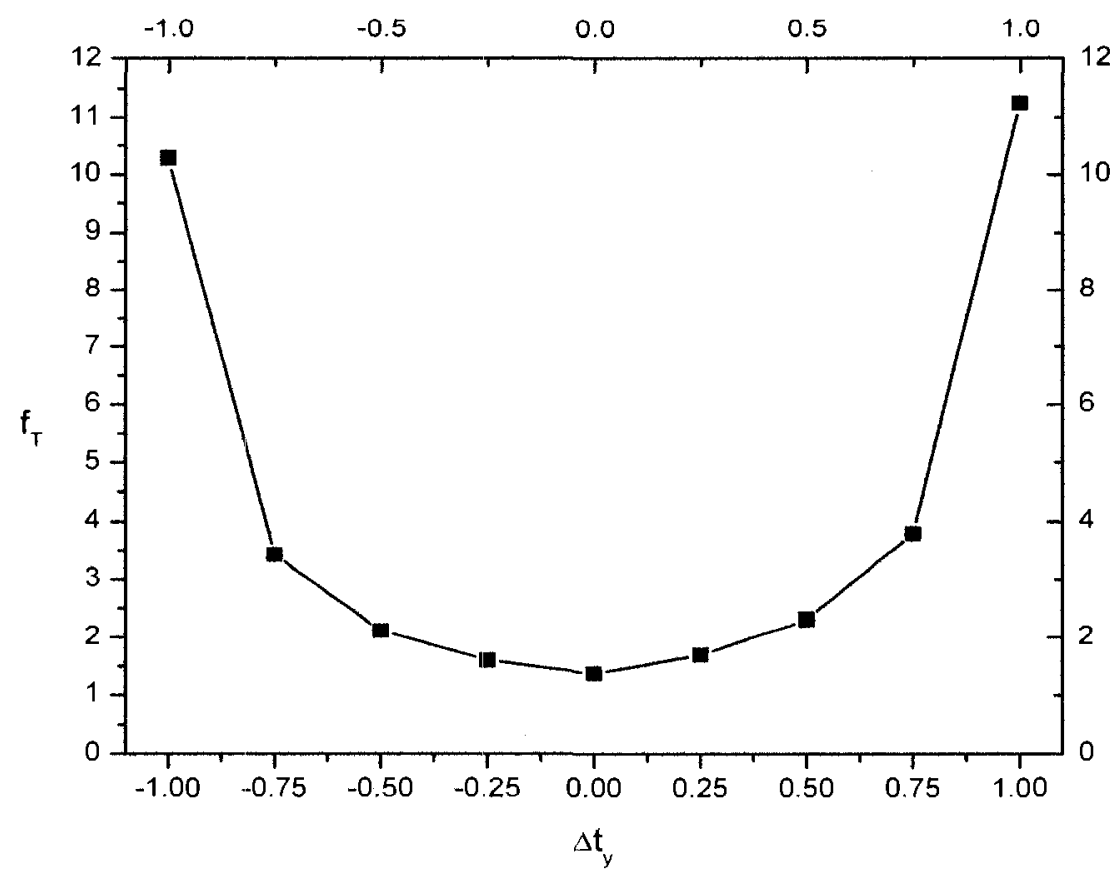

(e) $\phi=40^{\circ}$

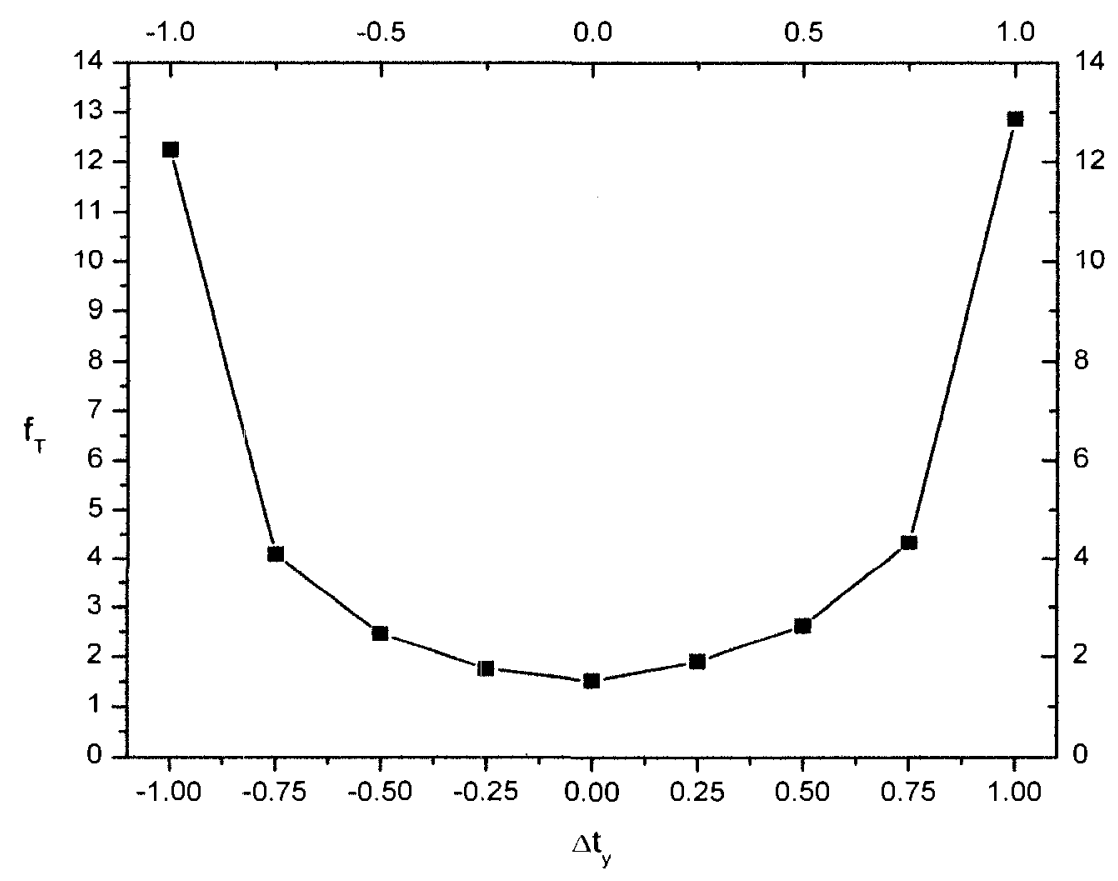

(f) $\phi=50^{\circ}$

Figure 5.1: Graphs of T-factor as a function of the normalized cyclic T-stress for various phase angles (e)-(f) 


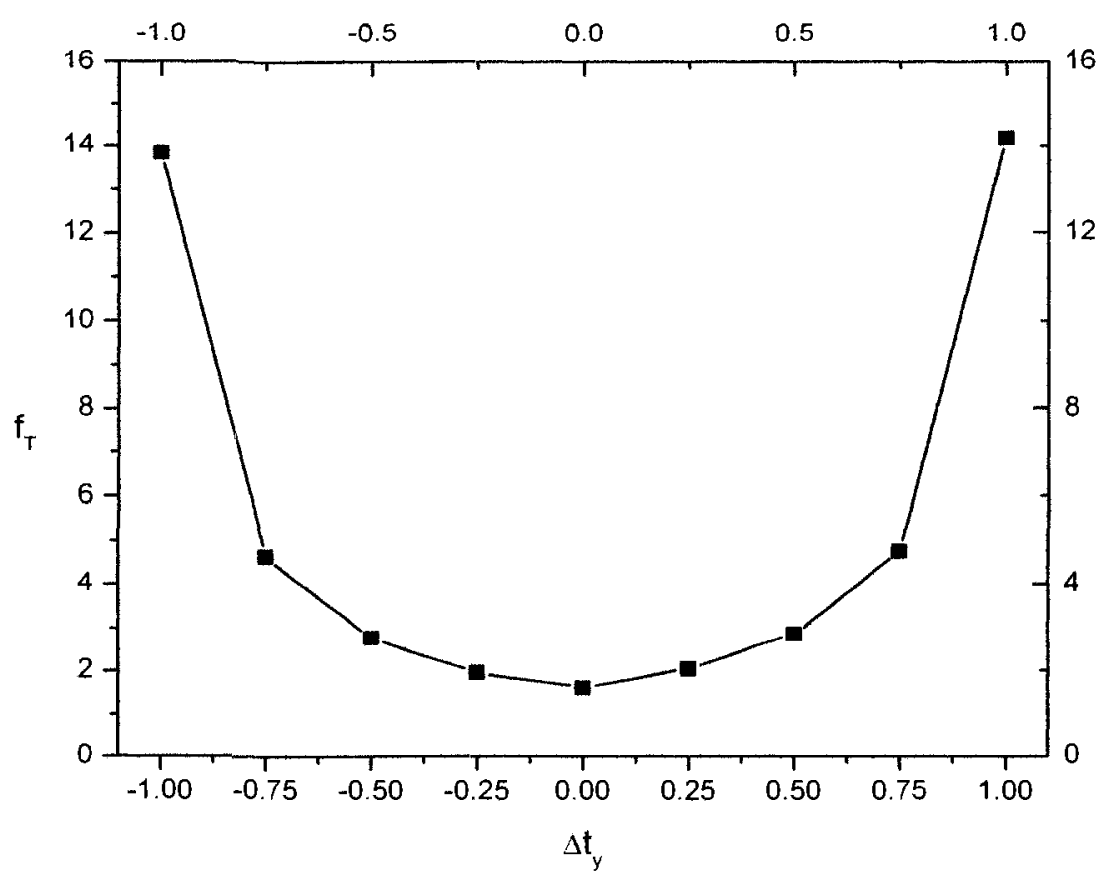

(g) $\phi=60^{\circ}$

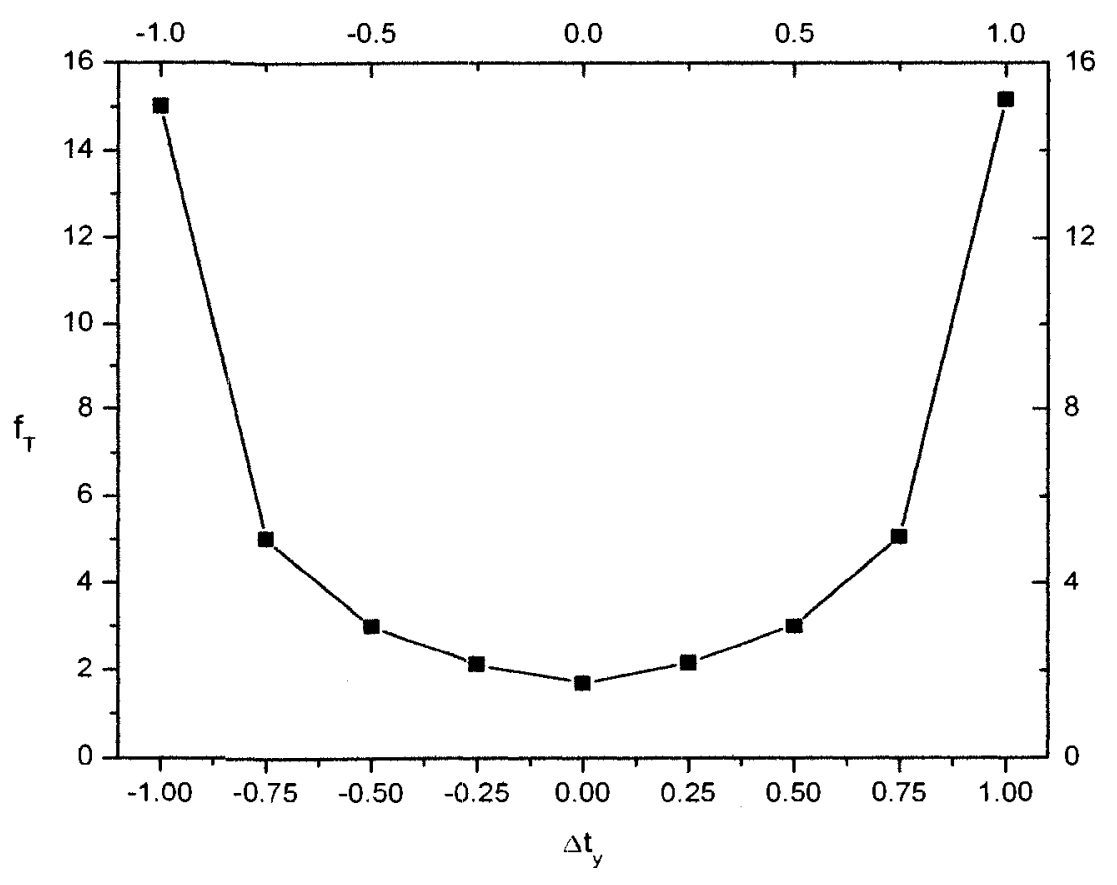

(h) $\phi=70^{\circ}$

Figure 5.1: Graphs of T-factor as a function of the normalized cyclic T-stress for various phase angles $(\mathrm{g})-(\mathrm{h})$ 


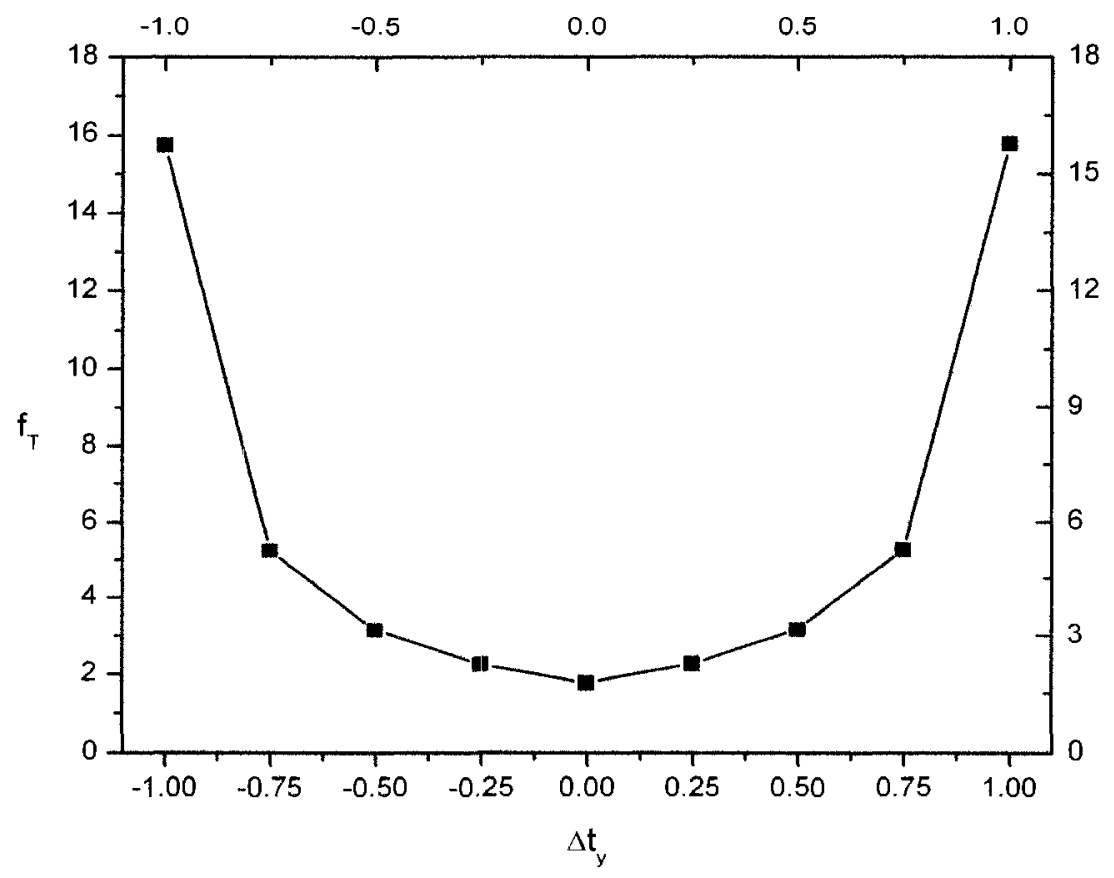

(i) $\phi=80^{\circ}$

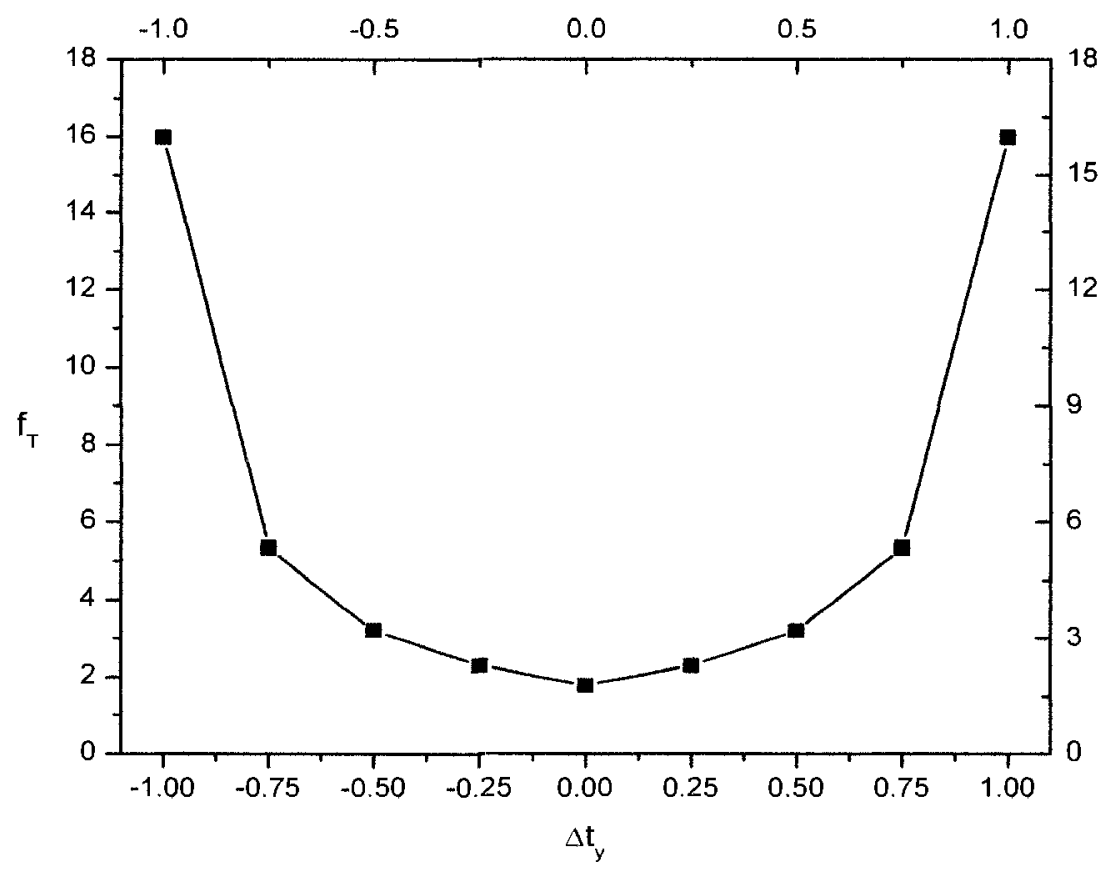

(j) $\phi=90^{\circ}$

Figure 5.1: Graphs of T-factor as a function of the normalized cyclic T-stress for various phase angles (i)-(j) 


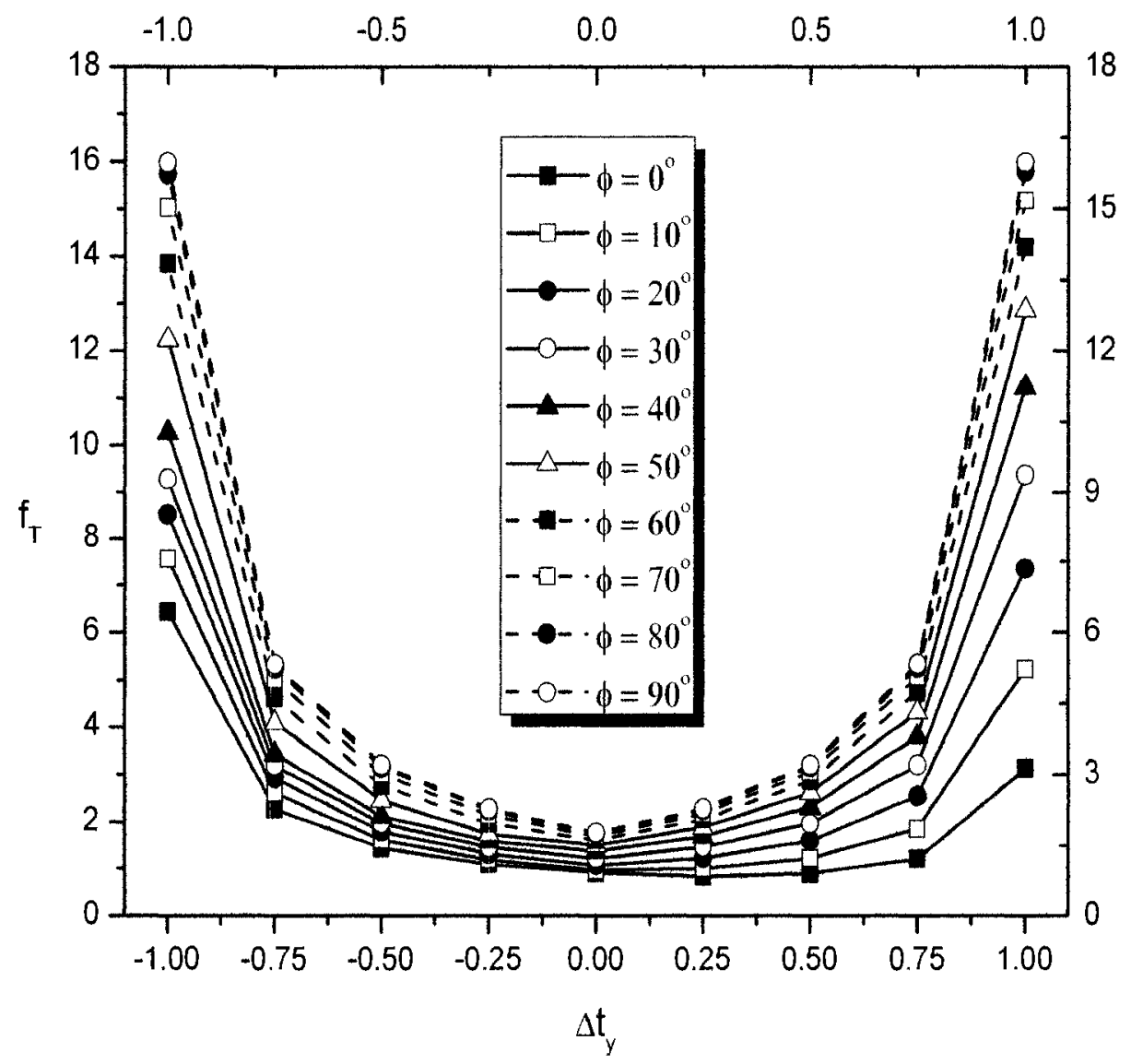

Figure 5.2: Combined graphs of T-factor as a function of the normalized cyclic T-stress for various phase angles 


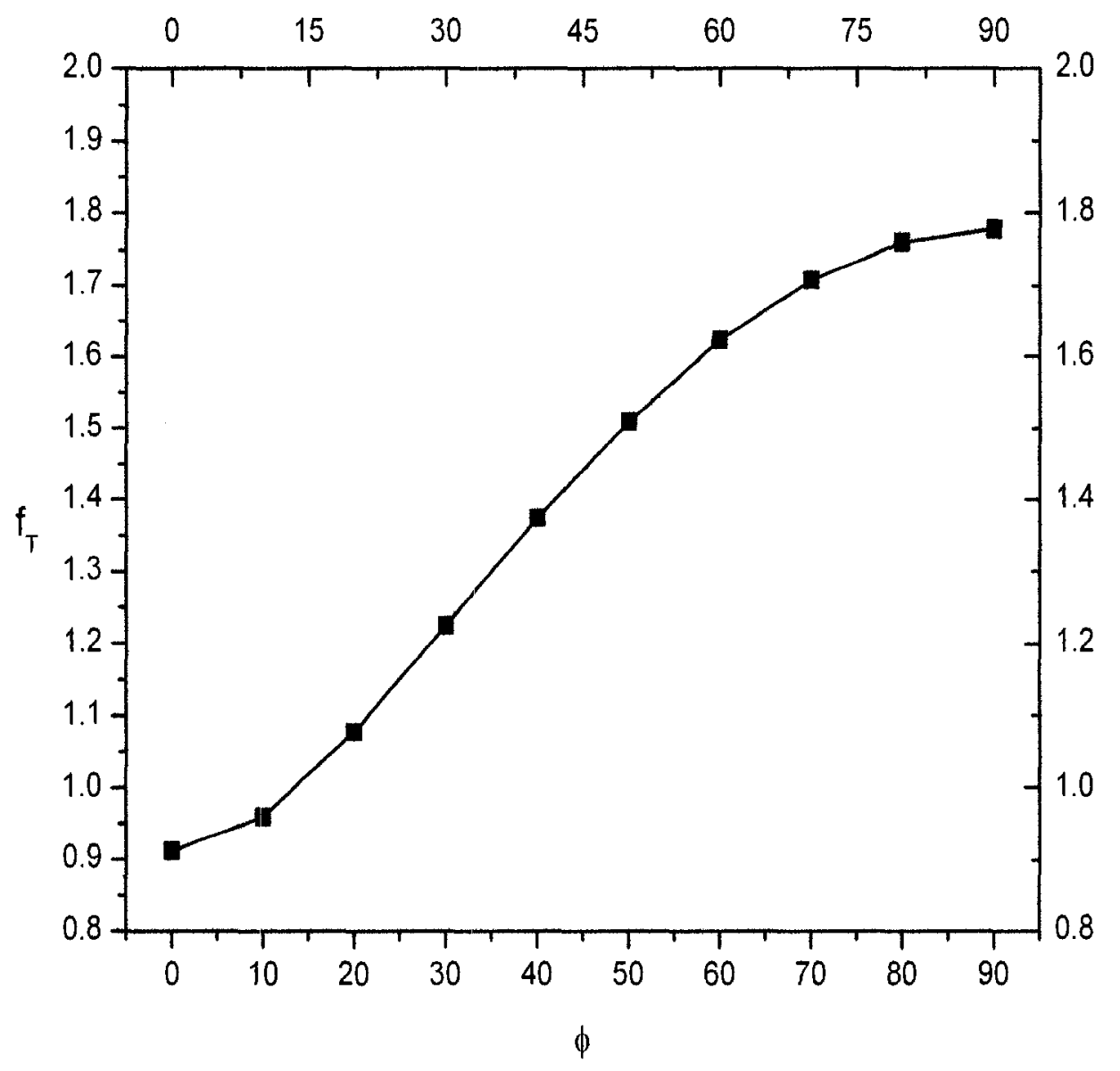

(a) $\Delta t_{y}=0$

Figure 5.3: Graphs of $\mathrm{T}$-factor as a function of phase angle for various normalized cyclic T-stress values (a) 


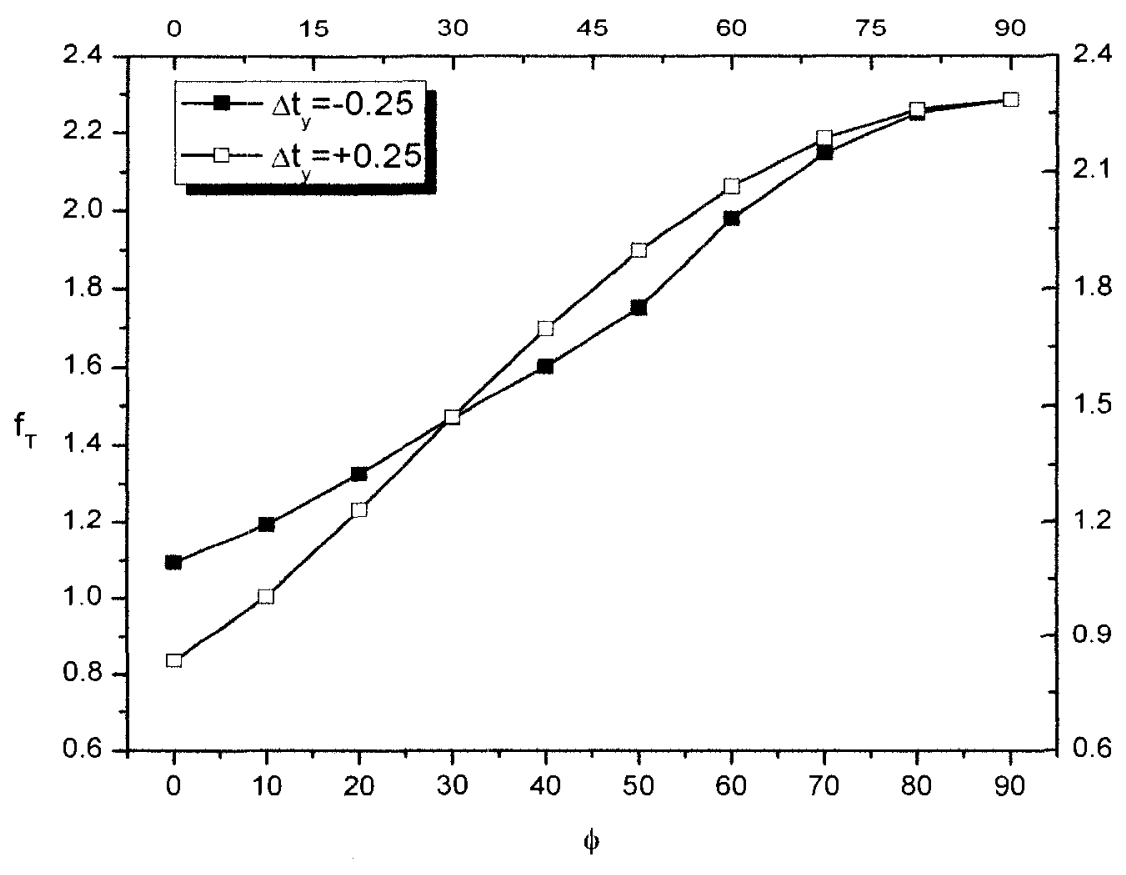

(b) $\Delta t_{y}= \pm 0.25$

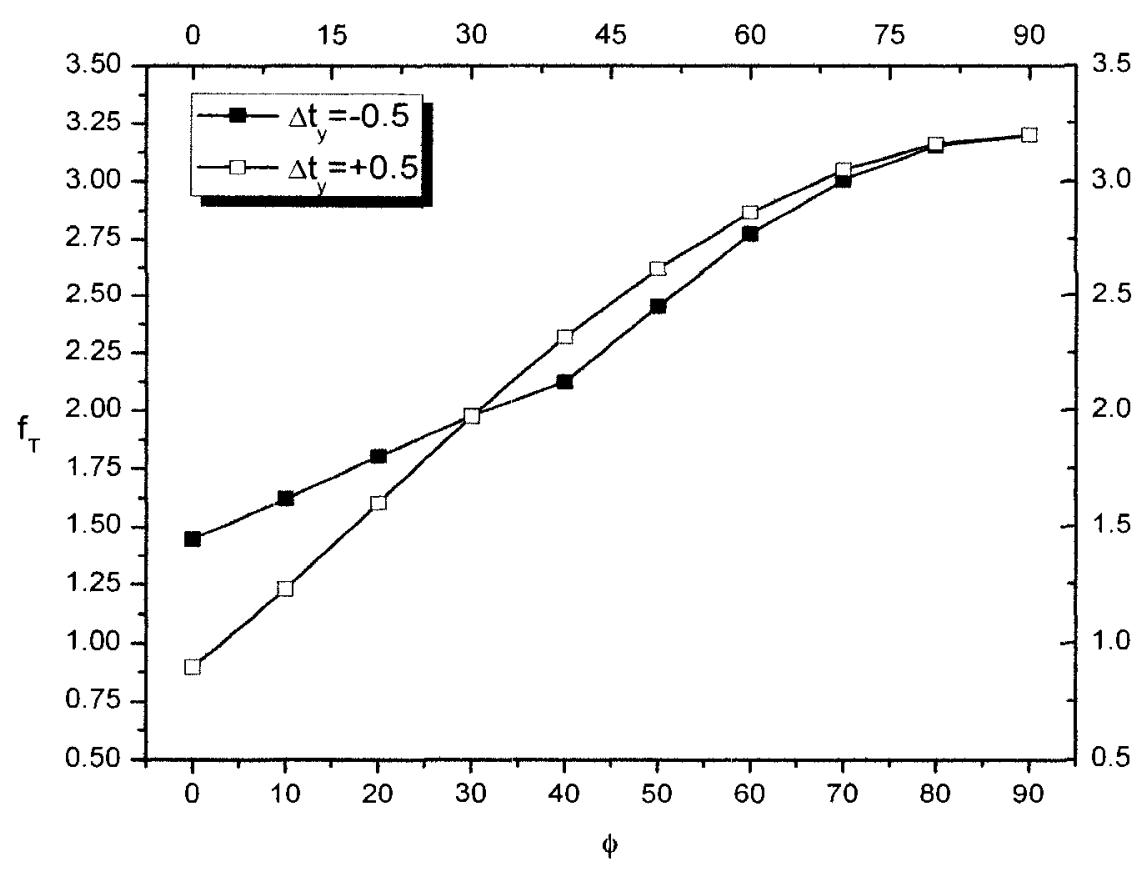

(c) $\Delta t_{y}= \pm 0.5$

Figure 5.3: Graphs of $\mathrm{T}$-factor as a function of phase angle for various normalized cyclic T-stress values (b)-(c) 


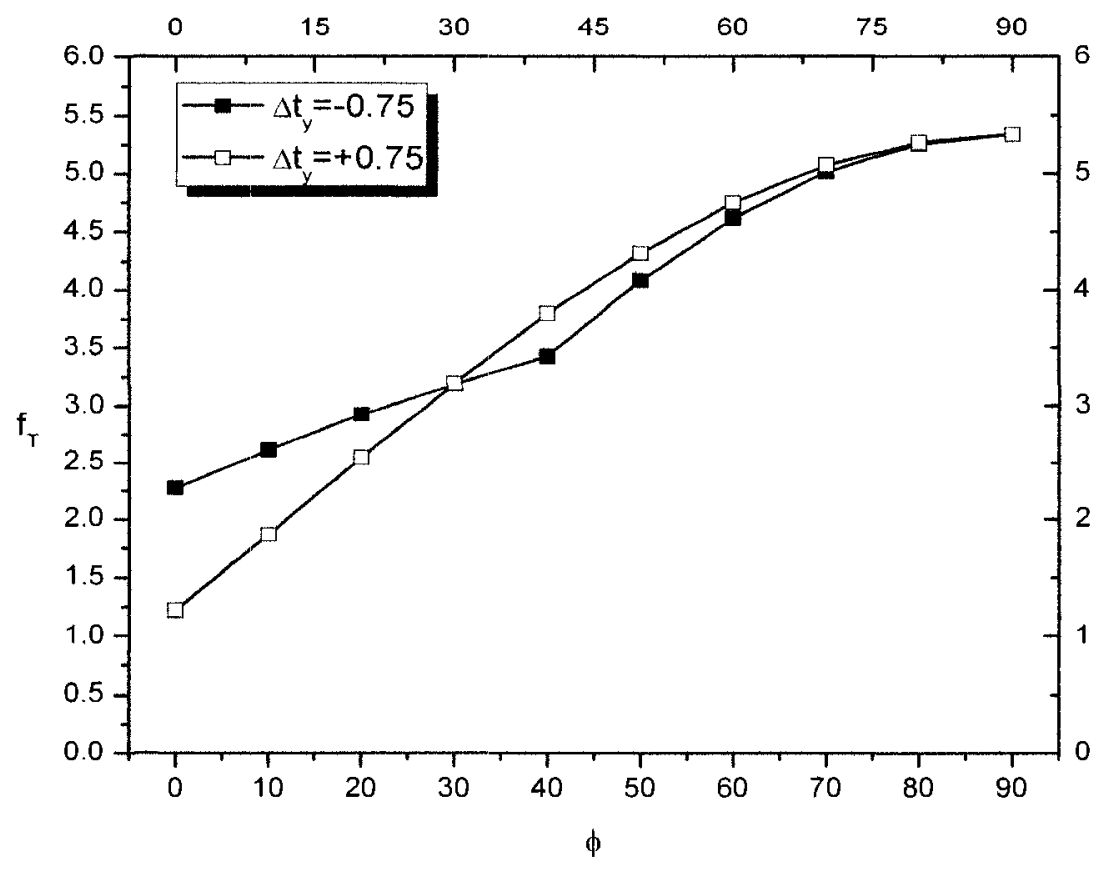

(d) $\Delta t_{y}= \pm 0.75$

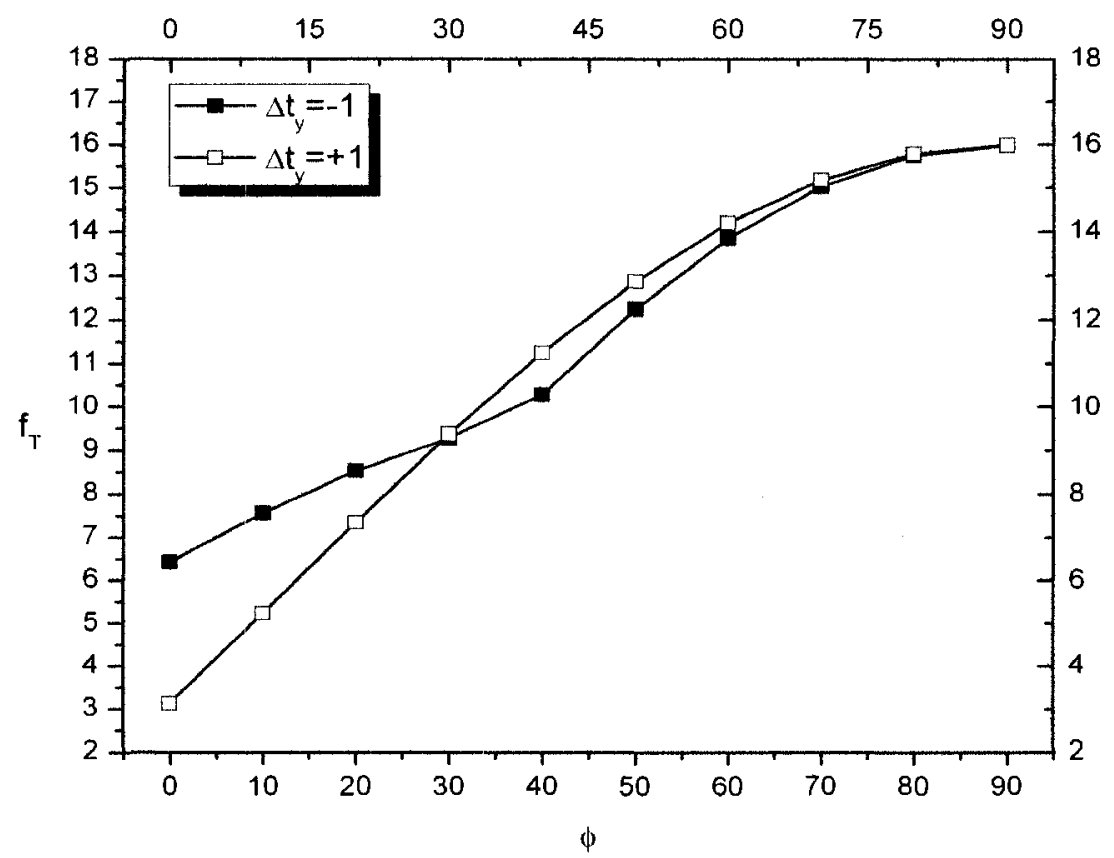

(e) $\Delta t_{y}= \pm 1$

Figure 5.3: Graphs of $\mathrm{T}$-factor as a function of phase angle for various normalized cyclic T-stress values (d)-(e) 


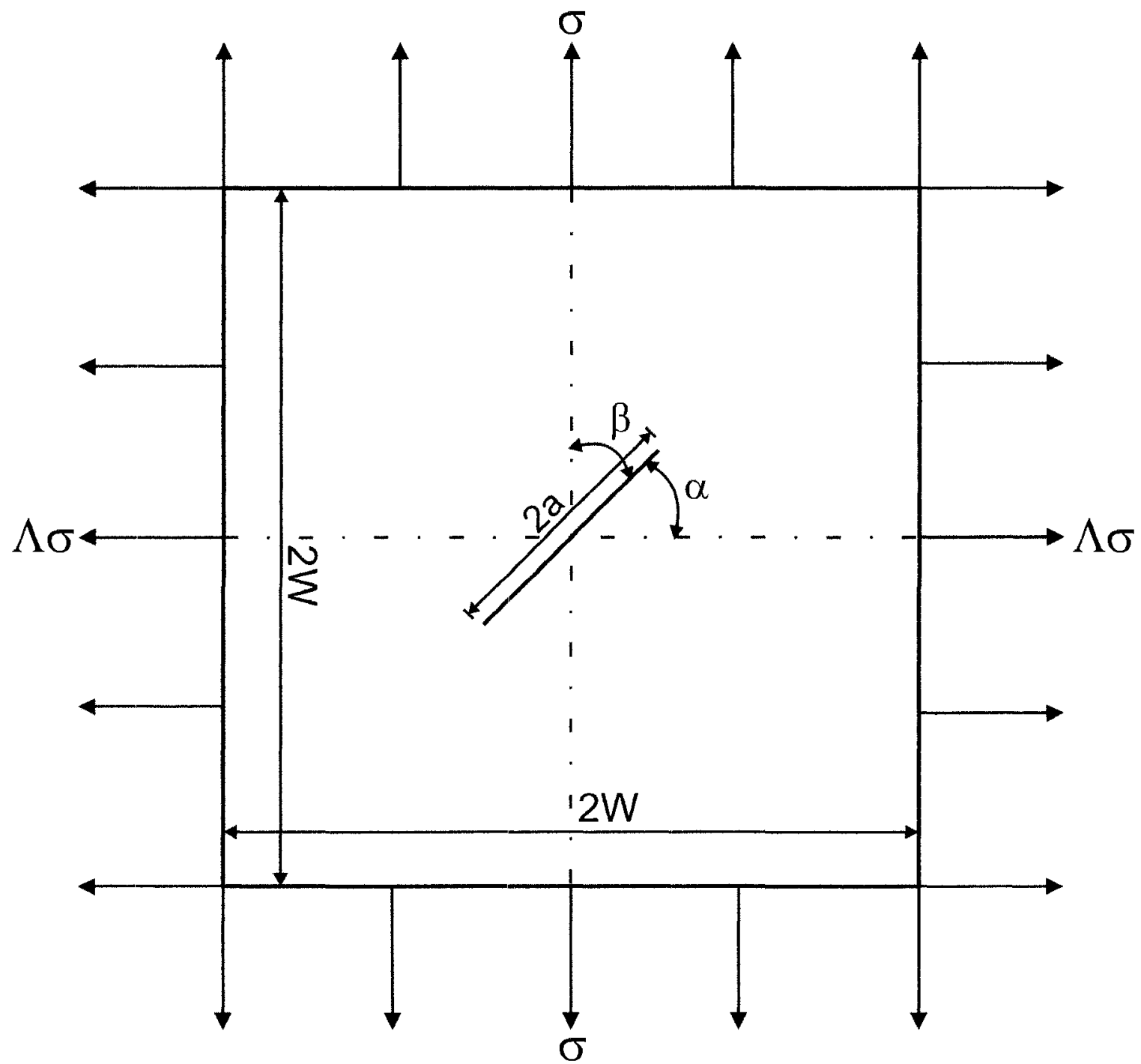

Figure 5.4: Bi-axially loaded CCP specimen that was used by Gao et al [1985] 


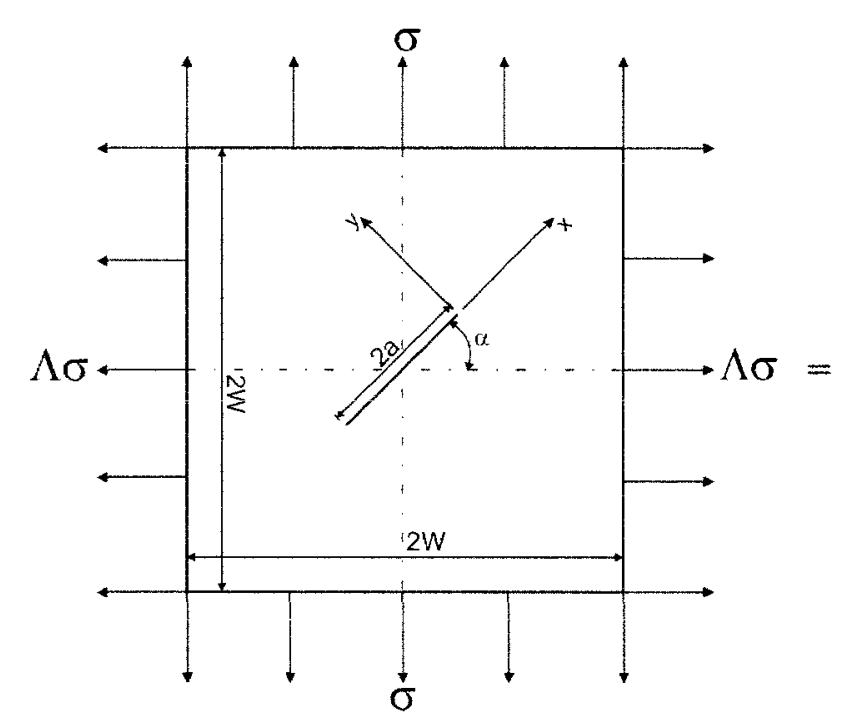

Bi-axial Loading

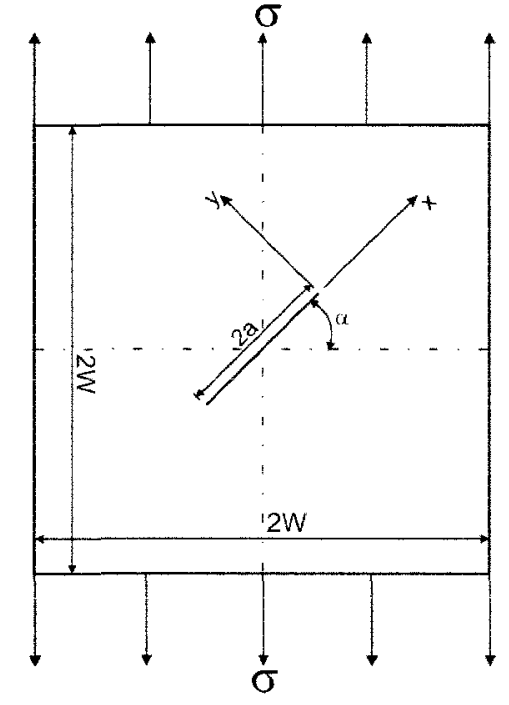

Longitudinal Loading

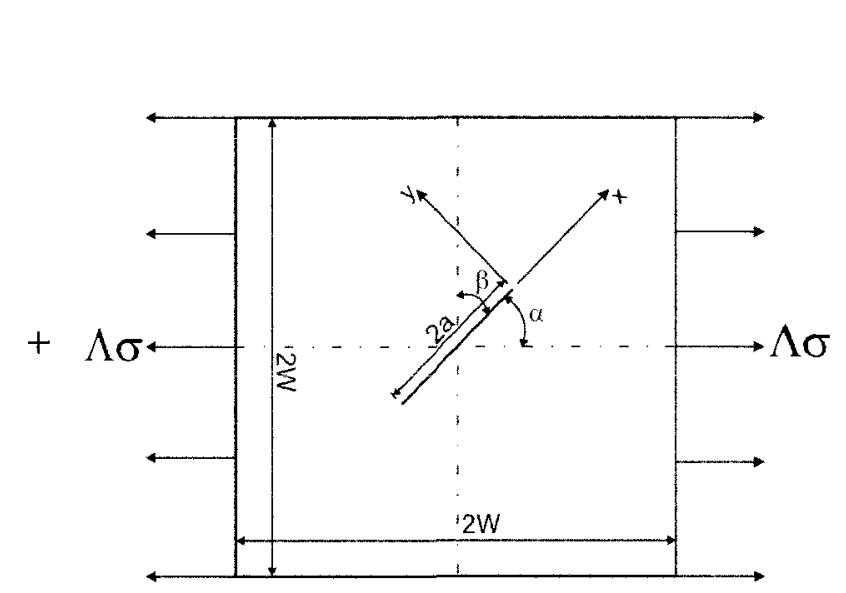

Transverse Loading

Figure 5.5a: Principle of superposition as applied to Gao et al's [1985] bi-axially loaded CCP specimen 


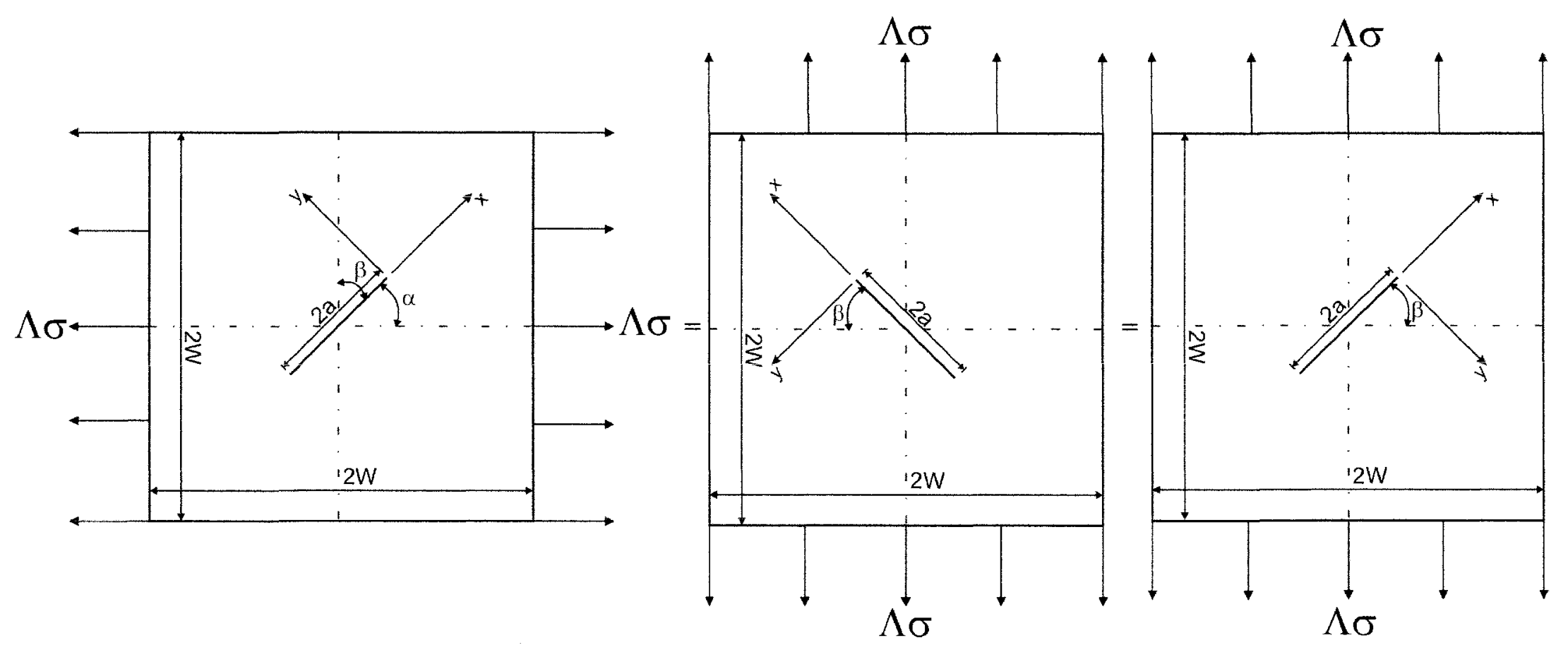

Figure 5.5b: Equivalent models of the transversely loaded component of the superposition principle as applied to the bi-axially loaded CCP in Figure 5.5a 


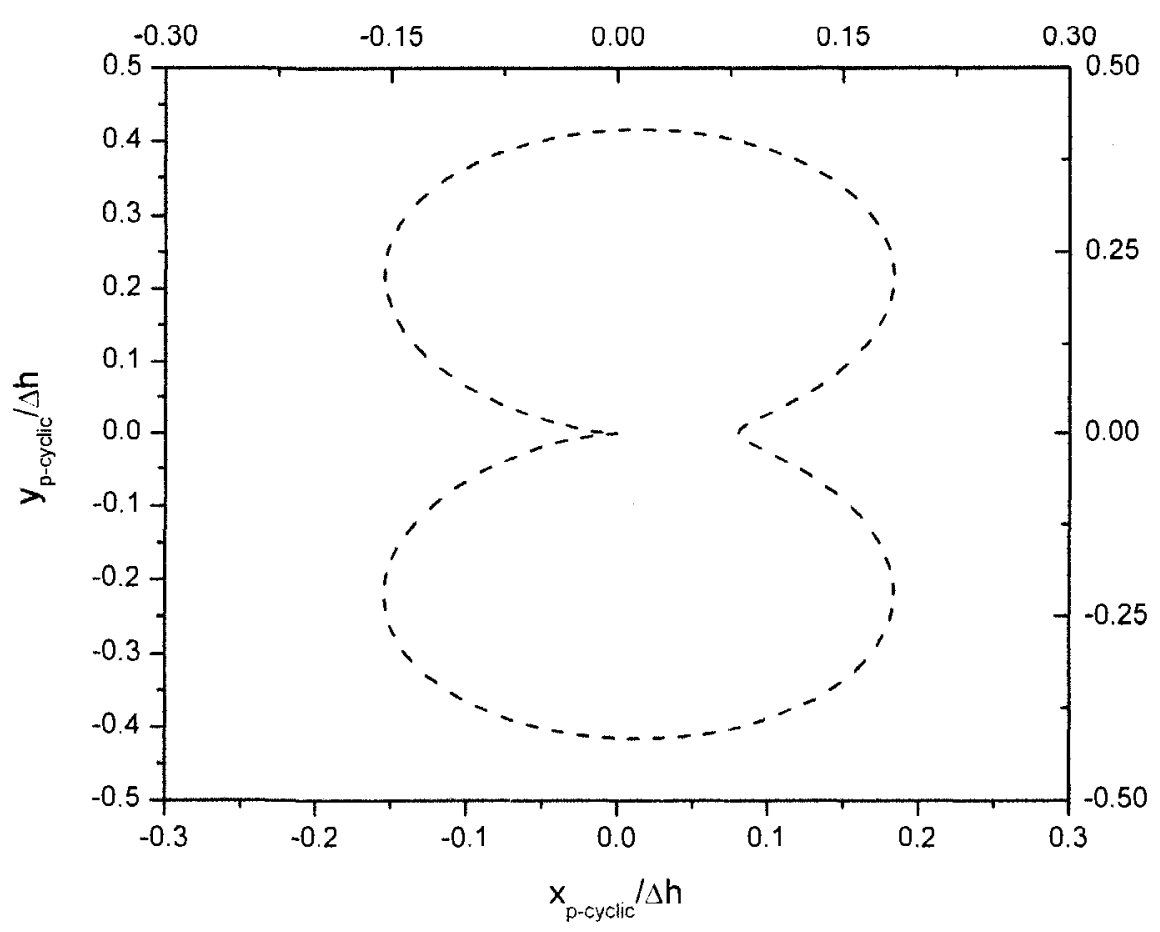

(a) Test 1: $\phi=0^{\circ}, \Delta T=0 M P a$

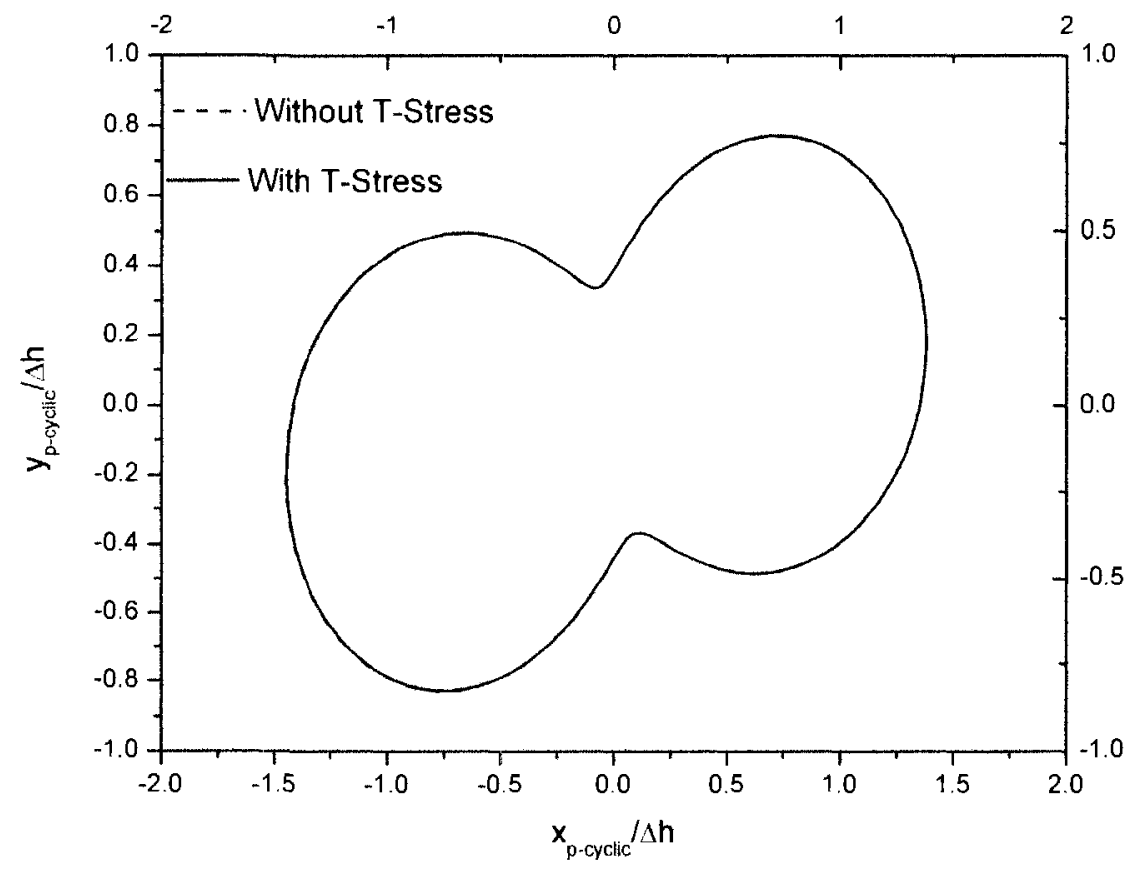

(b) Test 2: $\phi=71.2^{\circ}, \Delta T=-0.58 \mathrm{MPa}$

Figure 5.6: Normalized cyclic plastic zone maps calculated for Tests 1-5 of Gao et al's [1985] bi-axially loaded CCP specimen (a)-(b) 


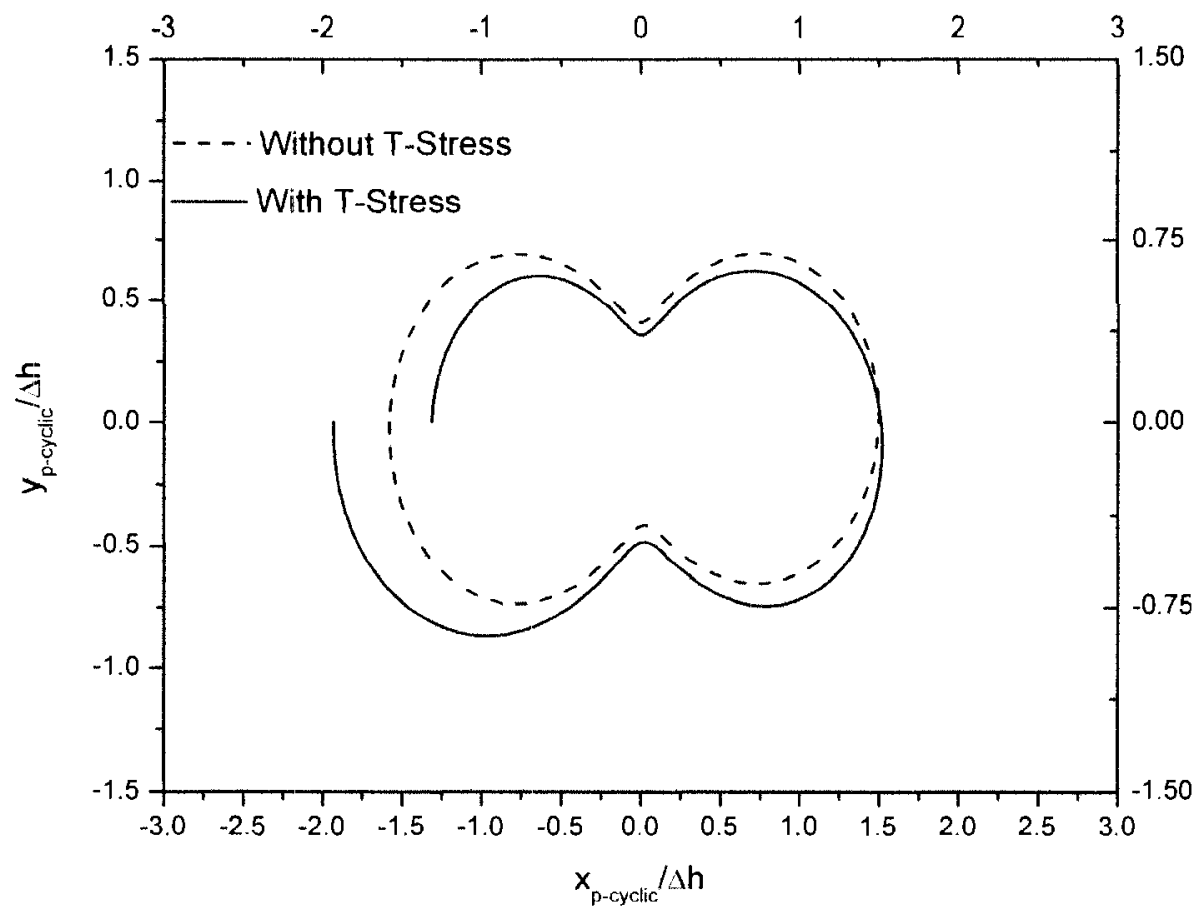

(c) Test 3: $\phi=87.5^{\circ}, \Delta T=53.56 \mathrm{MPa}$

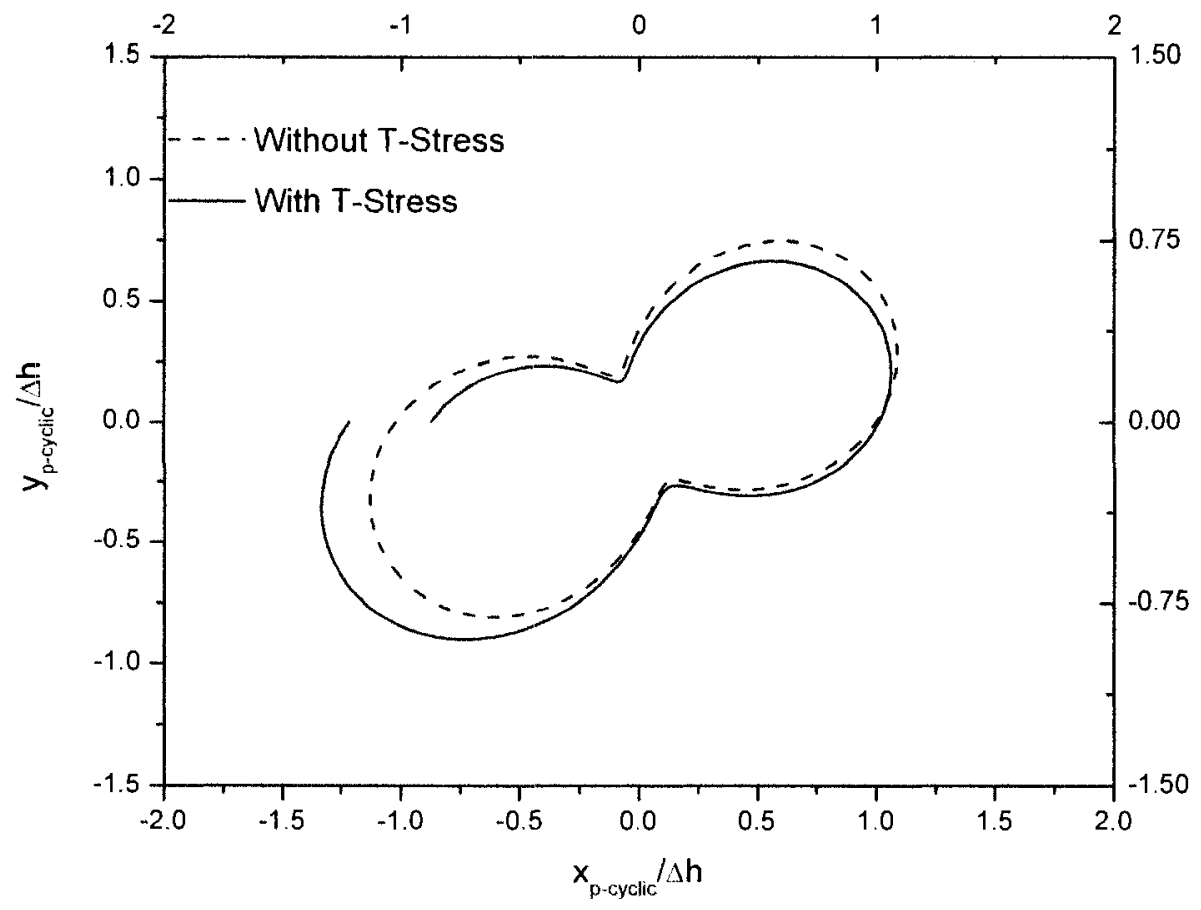

(d) Test 4: $\phi=53.5^{\circ}, \Delta T=45.97 M P a$

Figure 5.6: Normalized cyclic plastic zone maps calculated for Tests 1-5 of Gao et al's [1985] bi-axially loaded CCP specimen (c)-(d) 


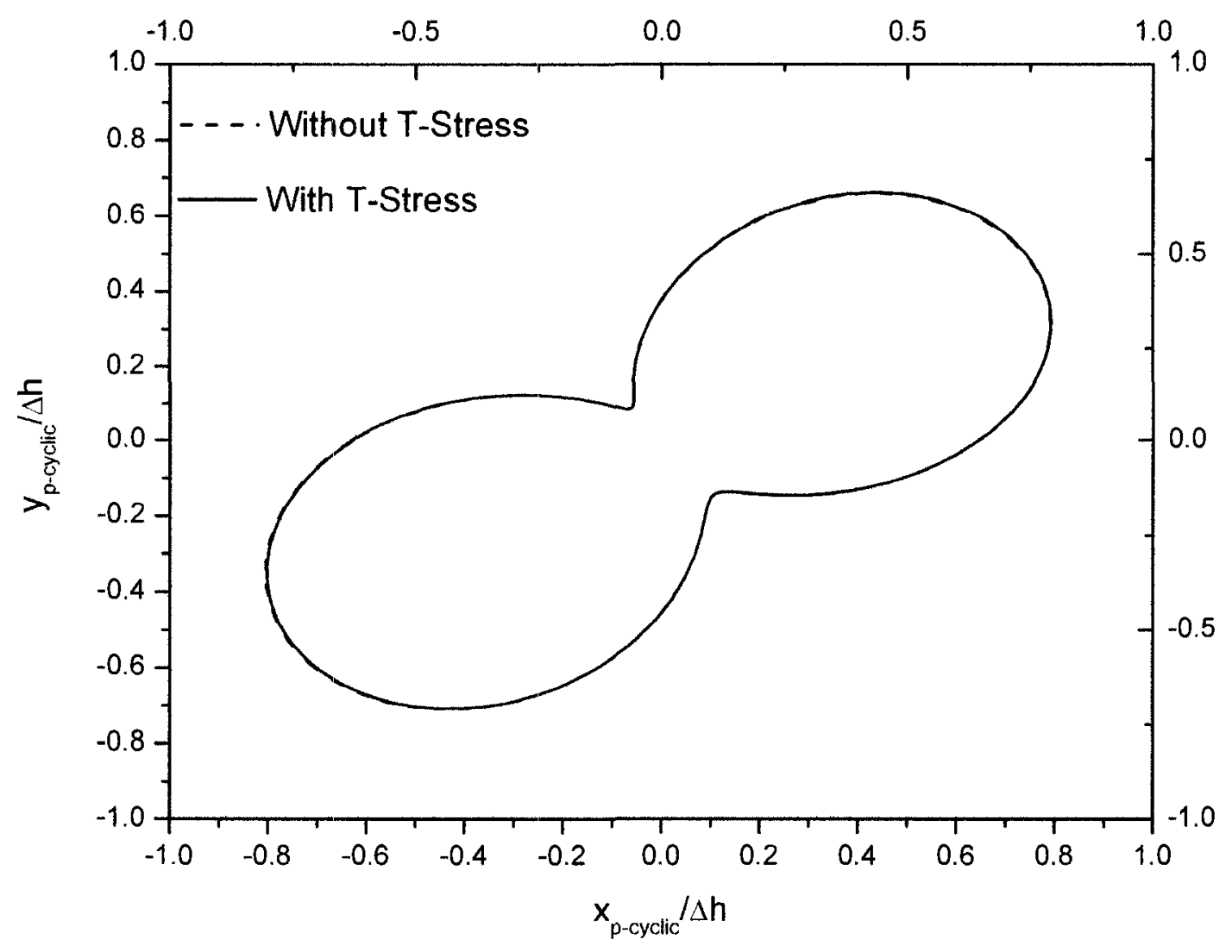

(e) Test 5: $\phi=39.1^{\circ}, \Delta T=-1.01 M P a$

Figure 5.6: Normalized cyclic plastic zone maps calculated for Tests 1-5 of Gao et al's [1985] bi-axially loaded CCP specimen (e) 


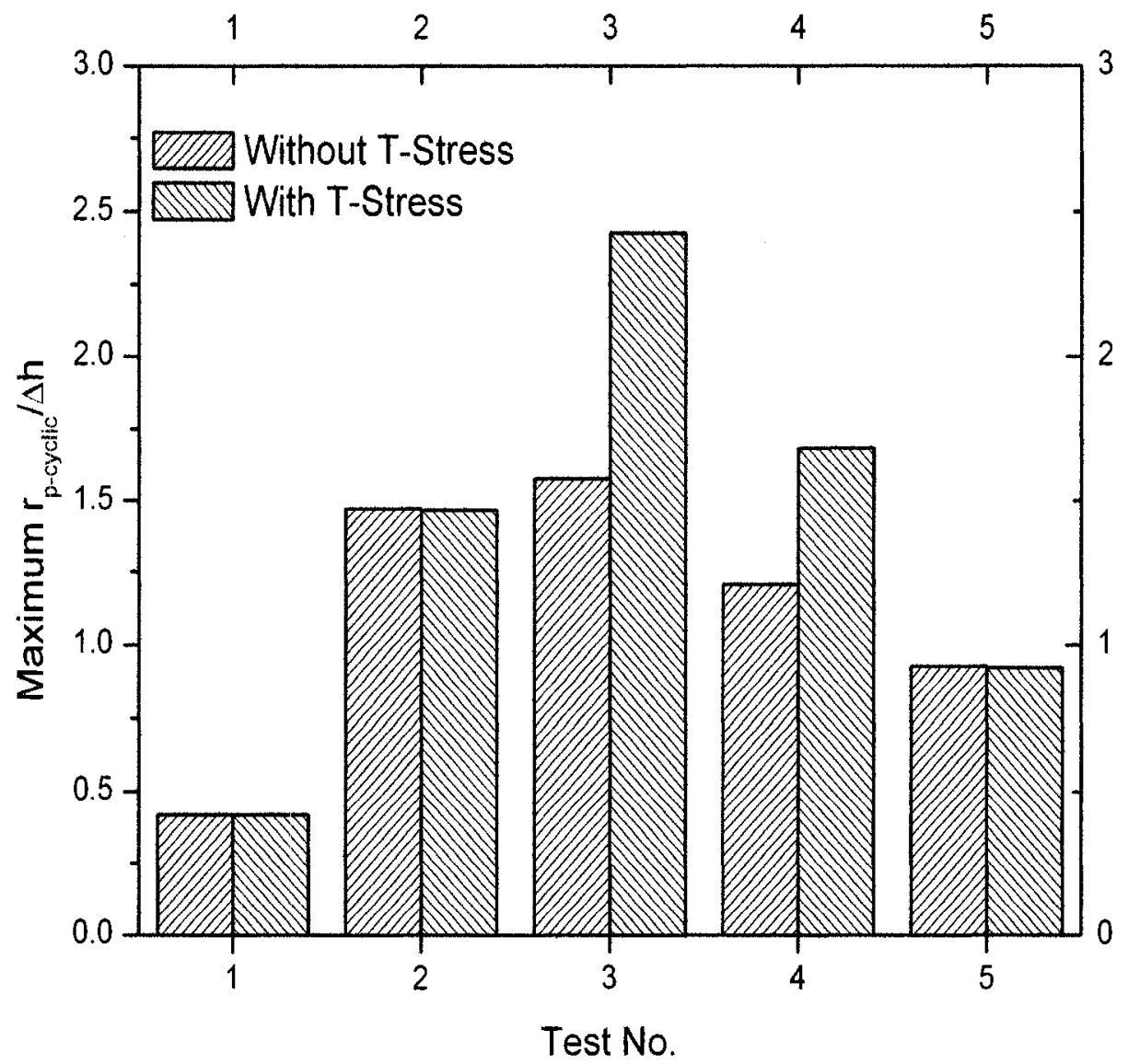

Figure 5.7: Comparison of maximum cyclic plastic zone size with and without $T$-stress for Tests $1-5$ of Gao et al's [1985] CCP specimen 


\section{Chapter 6}

\section{Conclusions and Recommendations}

The purpose of this thesis is to demonstrate the significance of T-stress: a constant stress acting parallel to the crack-front, in mixed-mode fatigue and fracture analysis. The thesis describes the parametric study conducted on center cracked plate (CCP) and singleedge cracked plate (SECP) specimens subjected to mixed-mode loading conditions. Also, the thesis describes the influence of the T-stress on the plastic zone size (PZS) under static and cyclic loading, as well as their effects on material fracture resistance and fatigue crack propagation rates.

\subsection{Conclusions}

Mixed-mode parametric studies using finite element analysis were conducted on a CCP and SECP specimen both of which are uniformly loaded longitudinally (Fig. 1.1). The purpose of the parametric study is to calculate the stress intensity factor (SIF) and Tstress values of both the CCP and SECP specimens for cracks of relative length 0.1-0.8 with respect to the specimen width and oriented between $0^{\circ}$ and $90^{\circ}$ with respect to the horizontal. The results of the study show that the T-stress is a significant factor that, in addition to the SIF, should be considered when analyzing fracture to achieve better accuracy.

An expression is developed to model the crack-tip plastic zone under static mixed-mode loading conditions that are influenced by T-stress. The plastic zone is then mapped and subsequently analyzed for mode II to mode I SIF ratios ranging from 0 to $\infty$, and normalized T-stress $\left(T / \sigma_{y}\right)$ values within the range of \pm 1 . The results show that the plastic zone rotates clockwise as the mode II to mode I SIF ratio increases, and that the size of the plastic zone increases as the absolute T-stress value becomes larger and the mode II loading condition becomes more dominant. Furthermore, the maximum PZS is found to be larger for positive T-stress values compared to negative $T$-stress values of equal magnitude only if the mode II to mode I SIF ratio is less than $0.577\left(\phi=30^{\circ}\right)$. 
An expression is then proposed relating the plastic zone under static loading conditions to material fracture resistance such that a material with a larger PZS at fracture exhibits a higher fracture resistance. From the fracture resistance expression, it was observed that for a given SIF, the fracture resistance increased with both the mode II to mode I SIF ratio and the absolute T-stress value, and that the fracture resistance for a given SIF is found to be lower for positive T-stress values compared to negative T-stress values of equal magnitude only if the mode II to mode I SIF ratio is less than 0.577 $\left(\phi=30^{\circ}\right)$.

Also, the plastic zone expression for static loading was adapted for cyclic loading conditions and then used to develop a relation for fatigue crack propagation rates. It was observed that for a given SIF, the fatigue crack propagation rates increased with both the mode II to mode I SIF ratio and the absolute T-stress value. The fatigue crack propagation rates for a given SIF are found to be lower for positive $\mathrm{T}$-stress values compared to negative T-stress values of equal magnitude only if the mode II to mode I SIF ratio is less than $0.577\left(\phi=30^{\circ}\right)$.

\subsection{Recommendations for Future Work}

Based on the research of this thesis, a number of recommendations are suggested:

1. T-stress and SIF values should be determined for a variety of different specimens and loading conditions. As such, the necessary T-stress and SIF data will be available to better analyze and predict fracture for "in-service" parts subjected to similar conditions.

2. In this thesis, a Poisson's ratio of 0.3 for steels is used. The plastic zone under both static and cyclic loading condition should be studied for other values of Poisson's ratio so that the necessary data (e.g. maximum PZS, T-factor, etc) are available to analyze different materials, as well as determine any trends in the plastic zone size with respect to Poisson's ratio. 
3. In the current study, it was proposed that both the crack-tip constraint and material fracture resistance could be characterized by the crack-tip plastic zone size. Further research should be conducted to confirm or refute the conclusions that were arrived at as a result of assuming a direct relation between the crack-tip plastic zone size and the both the crack-tip constraint and material fracture resistance.

4. It is also recommended that the crack propagation relation developed in Chapter 5 of this thesis be applied to actual testing data to determine its robustness at analyzing various mixed-mode fracture propagation problems.

5. Finally, it would be beneficial to study the plastic zone under mixed-mode loading condition influenced by $\mathrm{T}$-stress to determine how the direction in which a crack propagates may be affected by mixed-mode loading and the Tstress, thereby proving a means of better predicting the direction of crack propagation. 


\section{Appendix A}

\section{The Crack-tip Plastic Zone Approximation}

This Appendix contains the complete derivation for the approximation of the plastic zone around a crack-tip.

In constraint-based fracture mechanics using T-stress, for a two dimensional crack embedded in an isotropic linear elastic material subjected to mixed mode I and II planestrain loading conditions, the stress field around the crack tip (Fig. 2.3) is given by the following expressions (Chapter 2):

$$
\begin{aligned}
& \sigma_{x x}=\frac{K_{I}}{\sqrt{2 \pi r}} \cos \frac{\theta}{2} \cdot\left[1-\sin \frac{\theta}{2} \cdot \sin \frac{3 \theta}{2}\right]-\frac{K_{I I}}{\sqrt{2 \pi r}} \sin \frac{\theta}{2} \cdot\left[2+\cos \frac{\theta}{2} \cdot \cos \frac{3 \theta}{2}\right]+T \\
& \sigma_{y y}=\frac{K_{I}}{\sqrt{2 \pi r}} \cos \frac{\theta}{2} \cdot\left[1+\sin \frac{\theta}{2} \cdot \sin \frac{3 \theta}{2}\right]+\frac{K_{I}}{\sqrt{2 \pi r}} \sin \frac{\theta}{2} \cdot \cos \frac{\theta}{2} \cdot \cos \frac{3 \theta}{2} \\
& \sigma_{z z}=v\left(\sigma_{x x}+\sigma_{y y}\right)=2 v\left\{\frac{K_{I}}{\sqrt{2 \pi r}} \cos \frac{\theta}{2}-\frac{K_{I I}}{\sqrt{2 \pi r}} \sin \frac{\theta}{2}\right\}+v T \\
& \tau_{x y}=\frac{K_{I}}{\sqrt{2 \pi r}} \sin \frac{\theta}{2} \cdot \cos \frac{\theta}{2} \cdot \cos \frac{3 \theta}{2}+\frac{K_{I I}}{\sqrt{2 \pi r}} \cos \frac{\theta}{2} \cdot\left[1-\sin \frac{\theta}{2} \cdot \sin \frac{3 \theta}{2}\right] \\
& \tau_{y z}, \tau_{z x}=0
\end{aligned}
$$

where, $(r, \theta)$ are the polar coordinates and $(x, y)$ are the Cartesian coordinates both with origins at the crack tip (Fig. 2.3). $K_{\mathrm{I}}$ and $K_{\mathrm{II}}$ are the mode I and mode II stress intensity factors (SIF) respectively, and $v$ is Poisson's ratio. The $T$ term in Equation 2.4a is the elastic T-stress, which under plane strain loading conditions induces a stress equivalent to $v T$ in the z-direction. $\sigma$ and $\tau$ are the normal and shear stress respectively. 
The crack-tip-yielding zone, better known as the plastic zone, is by definition the region surrounding a crack tip that has yielded as a result of a localized stress field, which in this case is represented by Equation's 2.4a-e for plane strain conditions. As such, the plastic zone around a crack-tip can be estimated by employing the Von Mises yield criterion below [Shigley, 2003]:

$$
\left[\left(\sigma_{x x}-\sigma_{y y}\right)^{2}+\left(\sigma_{y y}-\sigma_{z z}\right)^{2}+\left(\sigma_{z z}-\sigma_{x x}\right)^{2}+6\left(\tau_{x y}^{2}+\tau_{y z}^{2}+\tau_{z x}^{2}\right)\right]=2 \sigma_{y}^{2}
$$

where, $\sigma_{y}$ is the material yield stress.

Equation 2.7 was used to determine the crack-tip yield zone by substituting into it Equations 2.4a-e and solving for $r=r_{y}$. The first step in solving for $r=r_{y}$ begins by determining the following terms in Equation 2.7:

$$
\left(\sigma_{x x}-\sigma_{y y}\right)^{2}=\left(\frac{2 K}{\sqrt{2 \pi r}}\left\{\cos \phi \cdot\left(\cos \frac{\theta}{2} \cdot\left[-\sin \frac{\theta}{2} \cdot \sin \frac{3 \theta}{2}\right]\right)-\sin \phi \cdot\left(\sin \frac{\theta}{2} \cdot\left[1+\cos \frac{\theta}{2} \cdot \cos \frac{3 \theta}{2}\right]\right)\right\}+T\right)^{2}
$$

$$
\begin{aligned}
\left(\sigma_{y y}-\sigma_{z z}\right)^{2}=\left(\frac { K } { \sqrt { 2 \pi r } } \left\{\cos \phi \cdot\left(\cos \frac{\theta}{2} \cdot\left[1+\sin \frac{\theta}{2} \cdot \sin \frac{3 \theta}{2}-2 v\right]\right)\right.\right. & \\
& \left.\left.+\sin \phi \cdot\left(\sin \frac{\theta}{2} \cdot\left[\cos \frac{\theta}{2} \cdot \cos \frac{3 \theta}{2}+2 v\right]\right)\right\}-v T\right)^{2}
\end{aligned}
$$

$$
\begin{aligned}
\left(\sigma_{z z}-\sigma_{x x}\right)^{2}=\left(\frac { K } { \sqrt { 2 \pi r } } \left\{\operatorname { c o s } \phi \cdot \left(\cos \frac{\theta}{2} \cdot[\right.\right.\right. & \left.\left.-1+\sin \frac{\theta}{2} \cdot \sin \frac{3 \theta}{2}+2 v\right]\right) \\
& \left.\left.+\sin \phi \cdot\left(\sin \frac{\theta}{2} \cdot\left[2+\cos \frac{\theta}{2} \cdot \cos \frac{3 \theta}{2}-2 v\right]\right)\right\}+\{v-1\} T\right)^{2}
\end{aligned}
$$


$6\left(\tau_{x y}\right)^{2}=6\left(\frac{K}{\sqrt{2 \pi r}}\left\{\cos \phi \cdot\left(\sin \frac{\theta}{2} \cdot \cos \frac{\theta}{2} \cdot \cos \frac{3 \theta}{2}\right)+\sin \phi \cdot\left(\cos \frac{\theta}{2} \cdot\left[1-\sin \frac{\theta}{2} \cdot \sin \frac{3 \theta}{2}\right]\right)\right\}\right)^{2}$

where,

$K=\sqrt{K_{I}^{2}+K_{I I}^{2}}$

$\phi=\tan ^{-1} \frac{K_{I I}}{K_{I}}$

and therefore,

$K_{I}=K \cos \phi$

$K_{I I}=K \sin \phi$

The term $K$, defined as the Net Stress Intensity Factor, represents the net combined effect of the mode I and mode II SIF's on the crack-tip, while $\phi$ represents the phase angle of the mode I and mode II SIF's where $\tan \phi$ denotes the mode II to mode I SIF ratio. Note, the boundary conditions of $K$ and $\phi$ are $K=K_{I}$ at $\phi=0^{\circ}$ for a pure mode I loading condition, and $K=K_{I I}$ at $\phi=90^{\circ}$ for a pure mode II loading condition.

Expanding, bringing together like-terms, and using trigonometric identities (Appendix C) to simplify Equations A1-A4 when adding them together transforms Equation 2.7 into:

$R^{2} K^{2}[a]+2 R K T[b]+T^{2}[c] \leq 2 \sigma_{y}^{2}$ 
where,

$$
\begin{aligned}
& R=\frac{1}{\sqrt{2 \pi r}} \text { (A6) } \\
& {[a]=\left[\cos ^{2} \phi \cdot\left\{\frac{3}{2} \sin ^{2} \theta+V(\cos \theta+1)\right\}+\cos \phi \cdot \sin \phi \cdot\{2 \sin \theta \cdot(3 \cos \theta-V)\}\right.} \\
&\left.+\sin ^{2} \phi \cdot\left\{6-\frac{9}{2} \sin ^{2} \theta-V(\cos \theta-1)\right\}\right]
\end{aligned}
$$

$[b]=\left[\cos \phi \cdot\left\{\cos \frac{\theta}{2} \cdot\left(-3\left\{\cos ^{2} \frac{\theta}{2}-\cos ^{2} \theta\right\}+V\right)\right\}+\sin \phi \cdot\left\{\sin \frac{\theta}{2} \cdot\left(-3\left\{\cos ^{2} \frac{\theta}{2}+\cos ^{2} \theta\right\}-V\right)\right\}\right]$

$[c]=\left[\frac{1}{2}\{3+V\}\right]$

and,

$V=(1-2 v)^{2}$

Setting the inequality in Equation A5 into an equality gives a formula for the boundary of the plastic zone, $r=r_{p}$ :

$$
\left.\left.R_{p}^{2} K^{2}[a]+2 R_{p} K T\right] b\right]+T^{2}[c]=2 \sigma_{y}^{2}
$$

where, $R_{p}=\frac{1}{\sqrt{2 \pi r_{p}}}$ 
dividing through by $2 \sigma_{y}^{2}$ and rearranging gives:

$$
R_{p}^{2}\left(\frac{K}{\sigma_{y}}\right)^{2} \frac{[a]}{2}+R_{p}\left(\frac{K}{\sigma_{y}}\right)\left(\frac{T}{\sigma_{y}}\right)[b]+\left(\frac{T}{\sigma_{y}}\right)^{2} \frac{[c]}{2}-1=0
$$

Equation A9 can be factored in two ways to yield two different quadratic equations, namely:

$$
\begin{aligned}
& R_{p}^{2}\left(\frac{K}{\sigma_{y}}\right)^{2}\left(\frac{\sigma_{y}}{T}\right)^{2} \frac{[a]}{2}+R_{p}\left(\frac{K}{\sigma_{y}}\right)\left(\frac{\sigma_{y}}{T}\right)[b]+\left[\frac{[c]}{2}-\left(\frac{\sigma_{y}}{T}\right)^{2}\right]=0 \\
& \frac{[a]}{2}+\frac{1}{R_{p}}\left(\frac{\sigma_{y}}{K}\right)\left(\frac{T}{\sigma_{y}}\right)[b]+\frac{1}{R_{p}^{2}}\left(\frac{\sigma_{y}}{K}\right)^{2}\left(\frac{T}{\sigma_{y}}\right)^{2}\left[\frac{[c]}{2}-\left(\frac{\sigma_{y}}{T}\right)^{2}\right]=0
\end{aligned}
$$

Equation A10b provides a simpler solution for $r=r_{p}$ than does Equation A10a. This is because the solution for $r_{p}$ provided by solving Equation Al0b is much simpler than that provided by Equation A10a. The solution provided by Equation A10b has a denominator that is independent of $\theta$, while the solution provided by Equation A10a has a complicated denominator dependent on $\theta$ and involves a square root. As such, for a given Poisson's ratio, material yield stress, and T-stress, the denominator in the quadratic formula for Equation A10b is constant for all $\theta$, thus making it easier to calculate the plastic zone compared to using equation A10a.

Applying the quadratic equation to Equation A10b yields the following solution:

$$
\frac{1}{R_{p}}\left(\frac{\sigma_{y}}{K}\right)\left(\frac{T}{\sigma_{y}}\right)=\frac{-[b] \pm \sqrt{[b]^{2}-4 \frac{[a]}{2}\left\{\frac{[c]}{2}-\left(\frac{\sigma_{y}}{T}\right)^{2}\right\}}}{2\left\{\frac{[c]}{2}-\left(\frac{\sigma_{y}}{T}\right)^{2}\right\}}
$$


substituting in Equation A8, factoring, and rearranging gives,

$r_{p}(\theta)=\frac{1}{2 \pi}\left(\frac{K}{\sigma_{y}}\right)^{2}\left[\frac{1}{c^{*}}\left\{-[b] t_{y} \pm \sqrt{[b]^{2} t_{y}^{2}-[a] c^{*}}\right\}\right]^{2}$

where,

$$
t_{y}=\frac{T}{\sigma_{y}}
$$

$c^{*}=[c] t_{y}^{2}-2$

The $t_{y}$ term is the normalized T-stress with respect to the material yield stress.

The next step for solving $r_{p}$ is to determine the nature of the \pm sign in Equation A12, which is accomplished using a test involving the core of Equation A12 denoted as $z_{c}:$

$z_{c}=\frac{1}{c^{*}}\left\{-[b] t_{y} \pm \sqrt{[b]^{2} t_{y}^{2}-[a] c^{*}}\right\}$

The core, $z_{c}$ is used so that the effects of squaring the core are avoided.

The test involves determining the value of $z_{c}$ in Equation A13 for $-180^{\circ} \leq \theta \leq 180^{\circ}$ (i.e. the region surrounding the crack-tip), $-1 \leq t_{y} \leq 1,0^{\circ} \leq \phi \leq 90^{\circ}$, and a Poisson"s ratio of 0.3 and $0^{\circ} \leq \phi \leq 90^{\circ}$. The results obtained for $\phi=20^{\circ}$ and $\phi=60^{\circ}$ are representative of all tests performed and are displayed in Tables A1 and A2. The results show that the largest positive value of $z_{c}$ is obtained by using the negative sign of the \pm sign. 
The solution for $r_{p}$ is finally given by:

$$
r_{p}(\theta)=\frac{1}{2 \pi}\left(\frac{K}{\sigma_{y}}\right)^{2}\left[\frac{1}{c^{*}}\left\{-[b] t_{y}-\sqrt{[b]^{2} t_{y}^{2}-[a] c^{*}}\right\}\right]^{2}
$$

where, $r_{p}$ represents the plastic zone.

Equation 4.1 provides approximations of the plastic zones for pure mode I, mixed mode I and mode II, and pure mode II loading conditions as used in Chapter 4 and Appendix B. Equation 4.1 demonstrates that the shape of the plastic zone as a function of $\theta$ is dependent on the value of $\phi, t_{y}$, and Poisson's ratio, while the size of the plastic zone as a function of $\theta$ is dependent on the value of the net SIF i.e. the combined net effect of the mode I and mode II SIF's. 
Table A1: Evaluation of the plus-minus-effect on the core $z_{c}$ for $\phi=20^{\circ}$

\begin{tabular}{|c|c|c|c|c|c|c|c|c|c|c|}
\hline \multirow{3}{*}{ Theta } & \multicolumn{10}{|c|}{ Normalized T-stress } \\
\hline & \multicolumn{2}{|c|}{-1} & \multicolumn{2}{|c|}{-0.5} & \multicolumn{2}{|c|}{0} & \multicolumn{2}{|c|}{0.5} & \multicolumn{2}{|c|}{1} \\
\hline & $\begin{array}{c}\text { Plus } \\
\text { Effect }\end{array}$ & $\begin{array}{l}\text { Minus } \\
\text { Effect }\end{array}$ & $\begin{array}{c}\text { Plus } \\
\text { Effect }\end{array}$ & $\begin{array}{l}\text { Minus } \\
\text { Effect }\end{array}$ & $\begin{array}{c}\text { Plus } \\
\text { Effect }\end{array}$ & $\begin{array}{l}\text { Minus } \\
\text { Effect }\end{array}$ & $\begin{array}{c}\text { Plus } \\
\text { Effect }\end{array}$ & $\begin{array}{l}\text { Minus } \\
\text { Effect }\end{array}$ & $\begin{array}{c}\text { Plus } \\
\text { Effect }\end{array}$ & $\begin{array}{l}\text { Minus } \\
\text { Effect }\end{array}$ \\
\hline$-180^{\circ}$ & -5.47 & 0.32 & -1.09 & 0.42 & -0.61 & 0.61 & -0.42 & 1.09 & -0.32 & 5.47 \\
\hline$-130^{\circ}$ & -6.11 & 0.86 & -1.57 & 0.88 & -1.05 & 1.05 & -0.88 & 1.57 & -0.86 & 6.11 \\
\hline$-90^{\circ}$ & -1.19 & 3.52 & -0.91 & 1.21 & -0.94 & 0.94 & -1.21 & 0.91 & -3.52 & 1.19 \\
\hline$-45^{\circ}$ & -0.63 & 1.73 & -0.47 & 0.61 & -0.48 & 0.48 & -0.61 & 0.47 & -1.73 & 0.63 \\
\hline $0^{\mathrm{o}}$ & -1.93 & 1.21 & -0.83 & 0.74 & -0.70 & 0.70 & -0.74 & 0.83 & -1.21 & 1.93 \\
\hline $45^{\circ}$ & -0.75 & 7.11 & -0.84 & 1.67 & -1.06 & 1.06 & -1.67 & 0.84 & -7.11 & 0.75 \\
\hline $90^{\circ}$ & -0.55 & 6.71 & -0.66 & 1.47 & -0.88 & 0.88 & -1.47 & 0.66 & -6.71 & 0.55 \\
\hline $135^{\circ}$ & -0.25 & 1.32 & -0.23 & 0.37 & -0.26 & 0.26 & -0.37 & 0.23 & -1.32 & 0.25 \\
\hline $180^{\circ}$ & -0.32 & 5.47 & -0.42 & 1.09 & -0.61 & 0.61 & -1.09 & 0.42 & -5.47 & 0.32 \\
\hline
\end{tabular}


Table A2: Evaluation of the plus-minus-effect on the core $z_{c}$ for $\phi=60^{\circ}$

\begin{tabular}{|c|c|c|c|c|c|c|c|c|c|c|}
\hline \multirow{3}{*}{ Theta } & \multicolumn{10}{|c|}{ Normalized T-stress } \\
\hline & \multicolumn{2}{|c|}{-1} & \multicolumn{2}{|c|}{-0.5} & \multicolumn{2}{|c|}{0} & \multicolumn{2}{|c|}{0.5} & \multicolumn{2}{|c|}{1} \\
\hline & $\begin{array}{c}\text { Plus } \\
\text { Effect }\end{array}$ & $\begin{array}{l}\text { Minus } \\
\text { Effect }\end{array}$ & $\begin{array}{c}\text { Plus } \\
\text { Effect }\end{array}$ & $\begin{array}{l}\text { Minus } \\
\text { Effect }\end{array}$ & $\begin{array}{c}\text { Plus } \\
\text { Effect }\end{array}$ & $\begin{array}{l}\text { Minus } \\
\text { Effect }\end{array}$ & $\begin{array}{c}\text { Plus } \\
\text { Effect }\end{array}$ & $\begin{array}{l}\text { Minus } \\
\text { Effect }\end{array}$ & $\begin{array}{c}\text { Plus } \\
\text { Effect }\end{array}$ & $\begin{array}{l}\text { Minus } \\
\text { Effect }\end{array}$ \\
\hline$-180^{\circ}$ & -13.8 & 0.82 & -2.77 & 1.07 & -1.54 & 1.54 & -1.07 & 2.77 & -0.82 & 13.8 \\
\hline$-130^{\circ}$ & -10.2 & 1.08 & -2.39 & 1.20 & -1.52 & 1.52 & -1.20 & 2.39 & -1.08 & 10.2 \\
\hline$-90^{\circ}$ & -3.73 & 1.15 & -1.24 & 0.90 & -0.95 & 0.95 & -0.90 & 1.24 & -1.15 & 3.73 \\
\hline$-45^{\circ}$ & -5.50 & 0.82 & -1.44 & 0.82 & -0.98 & 0.98 & -0.82 & 1.44 & -0.82 & 5.50 \\
\hline $0^{\circ}$ & -3.50 & 3.12 & -1.71 & 1.66 & -1.51 & 1.51 & -1.66 & 1.71 & -3.12 & 3.50 \\
\hline $45^{\circ}$ & -1.06 & 9.70 & -1.17 & 2.30 & -1.47 & 1.47 & -2.30 & 1.17 & -9.70 & 1.06 \\
\hline $90^{\circ}$ & -0.48 & 7.57 & -0.61 & 1.54 & -0.87 & 0.87 & -1.54 & 0.61 & -7.57 & 0.48 \\
\hline $135^{\circ}$ & -0.58 & 7.47 & -0.71 & 1.61 & -0.95 & 0.95 & -1.61 & 0.71 & -7.47 & 0.58 \\
\hline $180^{\circ}$ & -0.82 & 13.8 & -1.07 & 2.77 & -1.54 & 1.54 & -2.77 & 1.07 & -13.8 & 0.82 \\
\hline
\end{tabular}




\section{Appendix B}

\section{The Plastic Zone Discontinuity}

This Appendix contains the derivation of a mathematical model that is used to explain the occurrence of a discontinuity in the plastic zone across the crack-front in a number of mixed-mode applications involving $T$-stress.

The discontinuity in the plastic zone occurs across the crack-front i.e. at $\theta= \pm 180^{\circ}$. As such, the plastic zone will be examined explicitly at $\theta=-180^{\circ}$ and $\theta=180^{\circ}$ in order to determine the nature of the discontinuity.

For a two-dimensional crack embedded in an isotropic linear elastic material subjected to mixed mode I and II plain strain loading and T-stress, the general expressions for a stress field (Fig. 2.3) around the crack-tip are:

$$
\begin{aligned}
& \sigma_{x x}=\frac{K_{I}}{\sqrt{2 \pi r}} \cos \frac{\theta}{2} \cdot\left[1-\sin \frac{\theta}{2} \cdot \sin \frac{3 \theta}{2}\right]-\frac{K_{I}}{\sqrt{2 \pi r}} \sin \frac{\theta}{2} \cdot\left[2+\cos \frac{\theta}{2} \cdot \cos \frac{3 \theta}{2}\right]+T \\
& \sigma_{y y}=\frac{K_{I}}{\sqrt{2 \pi r}} \cos \frac{\theta}{2} \cdot\left[1+\sin \frac{\theta}{2} \cdot \sin \frac{3 \theta}{2}\right]+\frac{K_{I I}}{\sqrt{2 \pi r}} \sin \frac{\theta}{2} \cdot \cos \frac{\theta}{2} \cdot \cos \frac{3 \theta}{2} \\
& \sigma_{z z}=v\left(\sigma_{x x}+\sigma_{y y}\right)=2 v\left\{\frac{K_{I}}{\sqrt{2 \pi r}} \cos \frac{\theta}{2}-\frac{K_{I I}}{\sqrt{2 \pi r}} \sin \frac{\theta}{2}\right\}+v T \\
& \tau_{x y}=\frac{K_{I}}{\sqrt{2 \pi r}} \sin \frac{\theta}{2} \cdot \cos \frac{\theta}{2} \cdot \cos \frac{3 \theta}{2}+\frac{K_{I}}{\sqrt{2 \pi r}} \cos \frac{\theta}{2} \cdot\left[1-\sin \frac{\theta}{2} \cdot \sin \frac{3 \theta}{2}\right] \\
& \tau_{y z}, \tau_{z x}=0
\end{aligned}
$$


where, $(r, \theta)$ are the polar coordinates and $(x, y)$ are the Cartesian coordinates both with origins at the crack tip (Fig. 2.3). $K_{\mathrm{I}}$ and $K_{\mathrm{II}}$ are the mode I and mode II stress intensity factors (SIF) respectively, and $v$ is Poisson's ratio. The $T$ term in Equation 2.4a is the elastic $T$-stress, which under plane strain loading conditions induces a stress equivalent to $v T$ in the z-direction. $\sigma$ and $\tau$ are the normal and shear stress respectively.

At $\theta=-180^{\circ}$ the stress field (Eqn's 4.1a-e) becomes:

$$
\begin{aligned}
& \sigma_{x x}=\frac{2 K_{I I}}{\sqrt{2 J r}}+T \\
& \sigma_{y y}=0 \\
& \sigma_{z z}=v\left(\sigma_{x x}+\sigma_{y y}\right)=v\left(\sigma_{x x}\right)=v\left\{\frac{2 K_{I I}}{\sqrt{2 \pi r}}+T\right\} \\
& \tau_{x y}, \tau_{y z}, \tau_{z x}=0
\end{aligned}
$$

while at $\theta=180^{\circ}$ the stress field (Eqn's 4.1a-e) becomes:

$$
\begin{aligned}
& \sigma_{x x}=\frac{-2 K_{I I}}{\sqrt{2 \pi r}}+T \\
& \sigma_{y y}=0
\end{aligned}
$$

$\sigma_{z z}=v\left(\sigma_{x x}+\sigma_{y y}\right)=v\left(\sigma_{x x}\right)=v\left\{\frac{-2 K_{I I}}{\sqrt{2 \pi r}}+T\right\}$

$$
\tau_{x y}, \tau_{y z}, \tau_{z x}=0
$$


Note that, the stress field for $\theta=-180^{\circ}$ and $\theta=180^{\circ}$ are identical except for the difference in sign associated with the mode II SIF in $\sigma_{x x}$ and inherently $\sigma_{z z}$. Also, both stress fields share the same pattern i.e. $\sigma_{y y}=0, \sigma_{z z}=v\left(\sigma_{x x}\right)$ and $\tau_{x y}, \tau_{y z}, \tau_{z x}=0$.

To determine the location, $r=r_{y}$ where the material will just begin to yield at $\theta= \pm 180^{\circ}$, the Von Mises yield criterion below was used [Shigley, 2003]:

$$
\left[\left(\sigma_{x x}-\sigma_{y y}\right)^{2}+\left(\sigma_{y y}-\sigma_{z z}\right)^{2}+\left(\sigma_{z z}-\sigma_{x x}\right)^{2}+6\left(\tau_{x y}^{2}+\tau_{y z}^{2}+\tau_{z x}^{2}\right)\right]=2 \sigma_{y}^{2}
$$

where, $\sigma_{y}$ is the material yield stress.

To calculate the crack-tip yield point more efficiently the stress-field pattern shared by $\theta=-180^{\circ}$ and $\theta=180^{\circ}$ was made use of to reduce Equation 2.7 into:

$$
\left[\left(\sigma_{x x}\right)^{2}+\left(v \sigma_{x x}\right)^{2}+\left(\{v-1\} \sigma_{x x}\right)^{2}\right]=2 \sigma_{y}^{2}
$$

simplifying and rearranging Equation B9 gives:

$$
\frac{\sigma_{x x}^{2}}{\sigma_{y}^{2}} W-1=0
$$

where, $W=\left\{v^{2}-v+1\right\}$ 
The Point of Yielding at $\theta=-180^{\circ}$

For $\theta=-180^{\circ}$, substituting Equation $\mathrm{B} 1$ into Equation $\mathrm{B} 10$ and expanding yields:

$4 R_{p}^{2}\left(\frac{K_{I I}}{\sigma_{y}}\right)^{2} W+4 R_{p}\left(\frac{K_{I I}}{\sigma_{y}}\right)\left(\frac{T}{\sigma_{y}}\right) W+\left(\frac{T}{\sigma_{y}}\right)^{2} W-1=0$

where, $R_{p}=\frac{1}{\sqrt{2 \pi r_{p}}}$

factoring Equation $\mathrm{B} 11$ in the same manner as was done in Appendix A gives the following quadratic equation:

$4 W+4 W \frac{1}{R_{p}}\left(\frac{\sigma_{y}}{K_{I I}}\right)\left(\frac{T}{\sigma_{y}}\right)+\left\{W-\left(\frac{\sigma_{y}}{T}\right)^{2}\right\} \frac{1}{R_{p}^{2}}\left(\frac{\sigma_{y}}{K_{I I}}\right)^{2}\left(\frac{T}{\sigma_{y}}\right)^{2}=0$

solving Equation B12 using the quadratic formula gives:

$\frac{1}{R_{p}}\left(\frac{\sigma_{y}}{K_{I I}}\right)\left(\frac{T}{\sigma_{y}}\right)=\frac{-4 W \pm \sqrt{16 W^{2}-16 W\left\{W-\left(\frac{\sigma_{y}}{T}\right)^{2}\right\}}}{2\left\{W-\left(\frac{\sigma_{y}}{T}\right)^{2}\right\}}$

substituting in Equation A8, factoring, and rearranging gives the solution for $r_{p}$ at $\theta=-180^{\circ}$ :

$r_{p}(\theta=-180)=\frac{1}{2 \pi}\left(\frac{K_{I}}{\sigma_{y}}\right)^{2}\left[\frac{2}{\left\{t_{y}^{2} W-1\right\}}\left\{-t_{y} W \pm \sqrt{W}\right\}\right]^{2}$ 
where, $t_{y}=\frac{T}{\sigma_{y}}$ is the normalized T-stress

Determining the nature of the \pm sign in Equation B14 is the next step to solving for $r_{p}$ and is done by using a test involving the core of Equation B14 denoted as $z_{-180}$ below:

$z_{-180}=\frac{2}{\left\{t_{y}^{2} W-1\right\}}\left\{-t_{y} W \pm \sqrt{W}\right\}$

the core, $z_{-180}$ is used so that the effects of squaring the core are avoided.

The test involves determining the value of $z_{-180}$ in Equation B15 for $-1 \leq t_{y} \leq 1$, and a Poisson's ratio of 0.3 . The results obtained from the test are displayed in Table B1. Note that the core is independent of the phase angle, $\phi$. From the results it was determined that the largest positive value of $z_{-180}$ was achieved by using the negative sign of the \pm sign.

The final solution for the point of yielding $r_{p}$ at $\theta=-180^{\circ}$ is:

$r_{p}(\theta=-180)=\frac{1}{2 \pi}\left(\frac{K_{I}}{\sigma_{y}}\right)^{2}\left[\frac{2}{\left\{t_{y}^{2} W-1\right\}}\left\{-t_{y} W-\sqrt{W}\right\}\right]^{2}$ 


\section{The Point of Yielding at $\theta=180^{\circ}$}

The same procedure used to solve for $r_{p}$ at $\theta=-180^{\circ}$ is applied to solving for $r_{p}$ at $\theta=180^{\circ}$.

Substituting Equation B5 into Equation B10 for $\theta=180^{\circ}$ and expanding yields:

$4 R_{p}^{2}\left(\frac{K_{I I}}{\sigma_{y}}\right)^{2} W-4 R_{p}\left(\frac{K_{I I}}{\sigma_{y}}\right)\left(\frac{T}{\sigma_{y}}\right) W+\left(\frac{T}{\sigma_{y}}\right)^{2} W-1=0$

similar to what was done in Appendix A, Equation B17 was factored to give the following quadratic equation:

$4 W-4 W \frac{1}{R_{p}}\left(\frac{\sigma_{y}}{K_{I I}}\right)\left(\frac{T}{\sigma_{y}}\right)+\left\{W-\left(\frac{\sigma_{y}}{T}\right)^{2}\right\} \frac{1}{R_{p}^{2}}\left(\frac{\sigma_{y}}{K_{I I}}\right)^{2}\left(\frac{T}{\sigma_{y}}\right)^{2}=0$

using the quadratic formula to solve Equation B18 gives:

$$
\frac{1}{R_{p}}\left(\frac{\sigma_{y}}{K_{I I}}\right)\left(\frac{T}{\sigma_{y}}\right)=\frac{4 W \pm \sqrt{16 W^{2}-16 W\left\{W-\left(\frac{\sigma_{y}}{T}\right)^{2}\right\}}}{2\left\{W-\left(\frac{\sigma_{y}}{T}\right)^{2}\right\}}
$$

substituting in Equation $\mathrm{A} 8$ and Equation B19, factoring, and rearranging gives the solution for $r_{p}$ at $\theta=180^{\circ}$ :

$$
r_{p}\left(\theta=180^{\circ}\right)=\frac{1}{2 \pi}\left(\frac{K_{I I}}{\sigma_{y}}\right)^{2}\left[\frac{2}{\left\{t_{y}^{2} W-1\right\}}\left\{t_{y} W \pm \sqrt{W}\right\}\right]^{2}
$$


As was performed earlier for $\theta=-180^{\circ}$, the nature of the \pm sign in Equation B20 is determined using tests that involve evaluating the core $z_{180}$ below:

$$
z_{180}=\frac{2}{\left\{t_{y}^{2} W-1\right\}}\left\{t_{y} W \pm \sqrt{W}\right\}
$$

The core is evaluated at $-1 \leq t_{y} \leq 1$ for a Poisson's ratio of 0.3 . The results of the test are displayed in Table B2. Note again that the core is independent of the phase angle, $\phi$ and again, from the results it was determined that the largest positive value of $z_{180}$ was achieved by using the negative sign of the \pm sign.

The final solution for the point of yielding $r_{p}$ at $\theta=180^{\circ}$ is:

$$
r_{p}(\theta=180)=\frac{1}{2 \pi}\left(\frac{K_{I I}}{\sigma_{y}}\right)^{2}\left[\frac{2}{\left\{t_{y}^{2} W-1\right\}}\left\{t_{y} W-\sqrt{W}\right\}\right]^{2}
$$

The solution for the yield points, $r_{p}$ at $\theta=-180^{\circ}$ and $\theta=180^{\circ}$ as given by Equation B16 and Equation B22 respectively are identical in form with the only disparity being the difference in sign associated with the $t_{y} W$ term. In general, from both Equations B16 and B22, the yield point at $\theta= \pm 180^{\circ}$ is dependent only on the T-stress, the mode II SIF, and Poisson's ratio. More specifically, the yield point is not just dependent on the mode II SIF, but on the contribution of the mode II SIF to the normal stress in the x-direction, $\sigma_{x x}$.

To determine the difference in the position of the yield points at $\theta=-180^{\circ}$ and $\theta=180^{\circ}$ Equation B22 is subtracted from Equation B16 to yield:

$$
r_{p}(\theta=180)-r_{p}(\theta=-180)=\frac{1}{2 \pi}\left(\frac{K_{I I}}{\sigma_{y}}\right)^{2}\left[\frac{2}{\left\{t_{y}^{2} W-1\right\}}\right]^{2}\left[-4 t_{y} W^{3 / 2}\right]
$$


simplifying gives the following expression for the plastic zone boundary across the crackfront:

$$
r_{p}(\theta=180)-r_{p}(\theta=-180)=\frac{-8}{\pi}\left(\frac{K_{I}}{\sigma_{y}}\right)^{2} \frac{t_{y} W^{3 / 2}}{\left\{t_{y}^{2} W-1\right\}^{2}}
$$

The above expression has been dubbed the "dis/continuity" expression.

From Equation B30, the yield point at $\theta=-180^{\circ}$ is ahead of that at $\theta=180^{\circ}$ provided that the normalized T-stress is negative, and vice versa. Also, from Equation B30, the only way for the yield points at $\theta=-180^{\circ}$ and $\theta=180^{\circ}$ to coincide would be for either the T-stress and/or mode II SIF and/or the term W to be zero. However, according to Equation Bx, Poisson's ratio must be a complex number for $\mathrm{W}$ to be zero and since it is impossible for Poisson's ratio to be anything but a real number as it represents a physical material property, $\mathrm{W}$ can never be zero and can therefore never influence the continuity of the plastic zone boundary. The remaining terms of interest are the variables of T-stress and the mode II SIF. Hence, the conditions necessary to achieve yield point coincidence at $\theta= \pm 180^{\circ}$ would be to have a specimen subjected to pure mode I loading irrespective of the T-stress value, or to have a specimen subjected to zero $\mathrm{T}$-stress regardless of the mixed mode loading conditions. 
Table B1: Evaluation of the \pm -effect on the core $z_{-180}$ for $\theta=-180^{\circ}$ and Poisson's ratio 0.3

\begin{tabular}{cccccc}
\hline & \multicolumn{5}{c}{ Normalized T-stress $t_{y}$} \\
\cline { 2 - 6 } & -1 & -0.5 & 0 & 0.5 & 1 \\
\hline+ & -15.989 & -3.2000 & -1.7776 & -1.2307 & -0.9411 \\
- & 0.9411 & 1.2307 & 1.7776 & 3.2000 & 15.989 \\
\hline
\end{tabular}

Table B2: Evaluation of the \pm -effect on the core $z_{180}$ for $\theta=180^{\circ}$ and Poisson's ratio 0.3

\begin{tabular}{cccccc}
\hline & \multicolumn{5}{c}{ Normalized T-stress $t_{y}$} \\
\cline { 2 - 6 }+ & -1 & -0.5 & 0 & 0.5 & 1 \\
\hline & -0.9411 & -1.2307 & -1.7776 & -3.2000 & -15.989 \\
& 15.989 & 3.2000 & 1.7776 & 1.2307 & 0.9411 \\
\hline
\end{tabular}




\section{Appendix C}

\section{Trigonometric Identities}

In this Appendix, the trigonometric identities used in formulating the equations of Chapter 4 and Appendix A are derived and outlined.

Starting with three basic identities:

$$
\begin{aligned}
& \sin ^{2} \alpha+\cos ^{2} \alpha=1 \\
& \sin (\alpha \pm \beta)=\sin \alpha \cdot \cos \beta \pm \cos \alpha \cdot \sin \beta \\
& \cos (\alpha \pm \beta)=\cos \alpha \cdot \cos \beta \mp \sin \alpha \cdot \sin \beta
\end{aligned}
$$

Equations C1-3 will be used to create other identities that are helpful in simplifying and converting the functions used in Chapter 4 and Appendix A.

Taking Equation $\mathrm{C} 1$ and squaring gives:

$\left(\sin ^{2} \alpha+\cos ^{2} \alpha\right)^{2}=\sin ^{4} \alpha+2 \sin ^{2} \alpha \cdot \cos ^{2} \alpha+\cos ^{4} \alpha=1$

Setting $\alpha=\beta$ in Equation $\mathrm{C} 2$ gives:

$\sin (2 \alpha)=2 \sin \alpha \cdot \cos \alpha$

and squaring both sides of Equation C5 yields:

$$
\sin ^{2}(2 \alpha)=4 \sin ^{2} \alpha \cdot \cos ^{2} \alpha
$$


Setting $\alpha=\beta$ in Equation C3 gives:

$$
\cos (2 \alpha)=\cos ^{2} \alpha-\sin ^{2} \alpha
$$

employing Equation $\mathrm{C} 1$ in Equation $\mathrm{C} 7$ yields two more identities:

$$
\begin{aligned}
\cos (2 \alpha) & =\cos ^{2} \alpha-\left(1-\cos ^{2} \alpha\right)=2 \cos ^{2} \alpha-1 \\
& =\left(1-\sin ^{2} \alpha\right)-\sin ^{2} \alpha=1-2 \sin ^{2} \alpha
\end{aligned}
$$

Using Equations $\mathrm{C} 2$ to relate $\alpha$ and $\alpha / 2$ and $3 \alpha / 2$ gives:

$$
\begin{aligned}
& \sin \frac{\alpha}{2}=\sin \left(\alpha-\frac{\alpha}{2}\right)=\sin \alpha \cdot \cos \frac{\alpha}{2}-\cos \alpha \cdot \sin \frac{\alpha}{2} \\
& \sin \frac{3 \alpha}{2}=\sin \left(\alpha+\frac{\alpha}{2}\right)=\sin \alpha \cdot \cos \frac{\alpha}{2}+\cos \alpha \cdot \sin \frac{\alpha}{2}
\end{aligned}
$$

multiplying Equations $\mathrm{C} 10$ and $\mathrm{C} 11$ yields:

$$
\sin \frac{\alpha}{2} \cdot \sin \frac{3 \alpha}{2}=\sin ^{2} \alpha \cdot \cos ^{2} \frac{\alpha}{2}-\cos ^{2} \alpha \cdot \sin ^{2} \frac{\alpha}{2}
$$

and employing Equation $\mathrm{C} 1$ into Equation $\mathrm{C} 12$ gives:

$$
\sin \frac{\alpha}{2} \cdot \sin \frac{3 \alpha}{2}=\left(1-\cos ^{2} \alpha\right) \cdot \cos ^{2} \frac{\alpha}{2}-\cos ^{2} \alpha \cdot \sin ^{2} \frac{\alpha}{2}=\cos ^{2} \frac{\alpha}{2}-\cos ^{2} \alpha
$$


Using Equations $\mathrm{C} 3$ to relate $\alpha$ and $\alpha / 2$ and $3 \alpha / 2$ gives:

$$
\begin{aligned}
& \cos \frac{\alpha}{2}=\cos \left(\alpha-\frac{\alpha}{2}\right)=\cos \alpha \cdot \cos \frac{\alpha}{2}+\sin \alpha \cdot \sin \frac{\alpha}{2} \\
& \cos \frac{3 \alpha}{2}=\cos \left(\alpha+\frac{\alpha}{2}\right)=\cos \alpha \cdot \cos \frac{\alpha}{2}-\sin \alpha \cdot \sin \frac{\alpha}{2} \\
& \cos \alpha=\cos \left(\frac{3 \alpha}{2}-\frac{\alpha}{2}\right)=\cos \frac{\alpha}{2} \cdot \cos \frac{3 \alpha}{2}+\sin \frac{\alpha}{2} \cdot \sin \frac{3 \alpha}{2}
\end{aligned}
$$

multiplying Equations $\mathrm{C} 14$ and $\mathrm{C} 15$ yield:

$$
\cos \frac{\alpha}{2} \cdot \cos \frac{3 \alpha}{2}=\cos ^{2} \alpha \cdot \cos ^{2} \frac{\alpha}{2}-\sin ^{2} \alpha \cdot \sin ^{2} \frac{\alpha}{2}
$$

and employing Equation $\mathrm{C} 1$ into Equation $\mathrm{C} 17$ gives

$$
\cos \frac{\alpha}{2} \cdot \cos \frac{3 \alpha}{2}=\cos ^{2} \alpha \cdot\left(1-\sin ^{2} \frac{\alpha}{2}\right)-\sin ^{2} \alpha \cdot \sin ^{2} \frac{\alpha}{2}=\cos ^{2} \alpha-\sin ^{2} \frac{\alpha}{2}
$$

The trigonometric identities above denoted by ${ }^{*}$ were used directly in the derivations outlined in Chapter 4 and Appendix A. 


\section{References}

ABAQUS Theory Manual [2006] (v.6.6), Hibbitt, Karlsson \& Sorensen Inc.

ABAQUS/CAE Users Manual [2006] (v.6.6), Hibbitt, Karlsson \& Sorensen Inc.

T. L. Anderson [2005], Fracture Mechanics: Fundamentals and Applications ( $3^{\text {rd }}$ Ed.), Taylor \& Francis Group.

M. R. Ayatollahi, M. J. Pavier, \& D. J. Smith [1998], "Determination of T-stress from finite element analysis for mode I and mixed mode I/II loading", International Journal of Fracture, Vol. 91, pp. 283-298.

M. R. Ayatollahi, D. J. Smith, \& M. J. Pavier, [2002], "Crack-tip constraint in mode II deformation", International Journal of Fracture, Vol. 113, pp. 153-173.

P. P. Benham, R. J. Crawford, \& C. G. Armstrong [1996], Mechanics of Engineering Materials ( $2^{\text {nd }}$ Ed.), Prentice Hall.

O. L. Bowie [1973], "Solutions of plane crack problems by mapping technique", Mechanics of Fracture (Ed. G. C. Sih), Vol. 1, pp. 1-55.

Z.-Z. Du, C. Betegon, \& J. W. Hancock [1991], "J dominance in mixed mode loading", International Journal of F'racture, Vol. 52, pp. 191-206.

Z.-Z. Du \& J. W. Hancock [1991], “The effect of non-singular stresses on crack-tip constraint", Journal of the Mechanics and Physics of Solids, Vol. 39, pp. 555-567.

T. Fett [1998], "Technical Note: Stress intensity factors and T-stress in edge-cracked rectangular plates under mixed boundary conditions", Engineering Fracture Mechanics, Vol. 60, pp. 625-630. 
H. Gao, N. Alagok, M. W. Brown, \& K. J. Miller [1985], "Growth of fatigue cracks under combined mode I and mode II loads", Multiaxial Fatigue, ASTM STP 853, $K . J$. Miller \& M. W. Brown, Eds., American Society for Testing and Materials, pp. 184-202.

K. H. Huebner, D. L. Dewhirst, D. E. Smith, \& T. G. Byrom [2001], The Finite Element Method for Engineers ( $4^{\text {th }}$ Ed.), John Wiley \& Sons Inc.

P. Hutar, S. Seitl, \& Z. Knesl [2004], "Quantification of the effect of specimen geometry on the fatigue crack growth response by two-parameter fracture mechanics", Materials Science and Engineering, Vol. 387-389, pp. 491-494.

K.-J. Kang \& H. G. Beom [1991], "Plastic zone size near the crack tip in a constrained ductile layer under mixed mode loading", Engineering Fracture Mechanics, Vol. 66, pp. 257-268.

A. P. Kfouri [1986], "Some evaluation of the elastic T-term using Eshelby's method", International Journal of Fracture, Vol. 30, pp. 301-315.

Y. Kim, X. K. Zhu, \& Y. J. Chao [2001], "Quantification of constraint on elastic-plastic $3 \mathrm{D}$ crack front by the J-A $\mathrm{A}_{2}$ three-term solution", Engineering Fracture Mechanics, Vol. 68 , pp. 895-914.

H. Kitagawa \& R. Yuuki [1977], "Analysis of arbitrarily shaped crack in a finite plate using conformal mapping, $1^{\text {st }}$ Report - Construction of Analysis Procedure and its Applicability", Transactions of the Japan Society of Mechanical Engineers, Vol.43, pp. 4354-4362.

S. G. Larsson \& A. J. Carlsson [1973], "Influence of non-singular stress terms and specimen geometry on small-scale yielding at crack tips in elastic-plastic materials", Journal of the Mechanics and Physics of Solids, Vol. 21, pp. 263-277. 
H.-B Park, K.-M. Kim, \& B.-W. Lee [1996], "Plastic zone size in fatigue cracking", International Journal of Pressure Vessels and Piping, Vol. 68, pp. 279-285.

J. Qian \& A. Fatemi [1996], "Mixed mode fatigue crack growth: A Literature Survey", Engineering Fracture Mechanics, Vol. 55, pp. 969-990.

J. R. Rice [1974], "Limitations to the small scale yielding approximation for crack tip plasticity", Journal of the Mechanics and Physics of Solids, Vol. 22, pp. 17-26.

S. Seitl \& Z. Knesl [2008], "Two-parameter fracture mechanics: fatigue crack behavior under mixed mode conditions", Engineering Fracture Mechanics, Vol. 75, pp. 857-865.

A. S. Selvarathinam \& J. G. Goree [1998], "T-stress based fracture model for cracks in isotropic materials", Engineering Fracture Mechanics, Vol. 60, pp. 969-990.

T. L. Sham [1991], "The determination of elastic T-term using higher order weight functions", International Journal of Fracture, Vol. 48, pp. 81-102.

A. H. Sherry, C C. France, \& M. R. Goldthorpe [1995], "Compendium of T-stress solutions for two and three-dimensional cracked geometries", Fatigue and Fracture of Engineering Materials and Structures, Vol. 18, pp. 141-155.

J. Shigley, C. Mischke, \& R. Budynas [2003], Mechanical Engineering Design ( $7^{\text {th }}$ Ed.), McGraw-Hill.

D. J. Smith, M. R. Ayatollahi, \& M. J. Pavier [2001], "The role of T-stress in brittle fracture for linear elastic materials under mixed-mode loading", Fatigue and Fracture of Engineering Materials and Structures, Vol. 24, pp. 137-150. 
H. Y. Subramanya, S. Viswanath, \& R. Narasimhan [2005], "A three-dimensional numerical study of mixed mode (I and II) crack tip fields in elastic-plastic solids", International Journal of Fracture, Vol. 136, pp. 167-185.

K. Tanaka [1974], "Fatigue crack propagation from a crack inclined to the cyclic tensile axis", Engineering Fracture Mechanics, Vol. 6, pp. 493-507.

J. Tong [2002], "T-stress and its implications for crack growth", Engineering Fracture Mechanics, Vol. 69, pp. 1325-1337.

X. Wang [2002], "Elastic T-stress for cracks in test specimens subjected to non-uniform stress distributions", Engineering Fracture Mechanics, Vol. 69, pp. 1339-1352.

X. Wang, T. Lewis, \& R. Bell [2006], "Estimations of the T-stress for small cracks at notches", Engineering Fracture Mechanics, Vol. 73, pp. 366-375.

X. Wang \& Q. Gao [2007], "Effect of constraint on fatigue crack propagation in T-plate welded joints", Proceedings of OMAE2007: $26^{\text {th }}$ International Conference on Offshore Mechanics and Arctic Engineering, San Diego, USA.

M. L. Williams [1957], "On the stress distribution at the base of a stationary crack", ASME Journal of Applied Mechanics, Vol. 24, pp. 109-114.

W. K. Wilson [1971], "Numerical method for determining stress intensity factors of an interior crack in a finite plate", ASME Ser. D. Journal of Basic Engineering, Vol. 93, pp. 685-690.

H.-L. Yeh [2006], "Change of damage zone size by T-stress", Journal of Reinforced Plastics and Composites, Vol. 25, pp. 645-661. 